\title{
Modular Approaches to Diversified Soft Lewis Basic Complexants through Suzuki-Miyaura Cross-Coupling of Bromoheteroarenes with Organotrifluoroborates
}

\author{
Ai Lin Chin and Jesse D. Carrick* \\ Department of Chemistry, Tennessee Technological University, 55 University Drive, Cookeville,
} TN 38501

Table of Contents

S1-S3

Compound $6{ }^{1} \mathrm{H}$ NMR S4

Compound $6{ }^{13} \mathrm{C}$ NMR S5

Compound $11^{1} \mathrm{H}$ NMR S6

Compound $11^{13} \mathrm{C}$ NMR S7

Compound $12{ }^{1} \mathrm{H}$ NMR S8

Compound $12{ }^{13} \mathrm{C}$ NMR S9

Compound $13{ }^{1} \mathrm{H}$ NMR S10

Compound $13{ }^{13} \mathrm{C}$ NMR S11 Compound $14{ }^{1} \mathrm{H}$ NMR S12

Compound $14{ }^{13} \mathrm{C}$ NMR S13

Compound $15{ }^{1} \mathrm{H}$ NMR S14

Compound $15{ }^{13} \mathrm{C}$ NMR S15

Compound $16{ }^{1} \mathrm{H}$ NMR S16 Compound $16{ }^{13} \mathrm{C}$ NMR S17

Compound $17^{1} \mathrm{H}$ NMR S18

Compound $17{ }^{13} \mathrm{C}$ NMR S19 Compound $18{ }^{1} \mathrm{H}$ NMR S20 Compound $18{ }^{13} \mathrm{C}$ NMR S21 Compound $19{ }^{1} \mathrm{H}$ NMR S22

Compound $19{ }^{13} \mathrm{C}$ NMR S23

Compound $20{ }^{1} \mathrm{H}$ NMR

S24

Compound $20{ }^{13} \mathrm{C}$ NMR S25 Compound $21^{1} \mathrm{H}$ NMR S26 Compound $21{ }^{13} \mathrm{C}$ NMR S27

Compound $22{ }^{1} \mathrm{H}$ NMR Compound $22{ }^{13} \mathrm{C}$ NMR S28 Compound $23{ }^{1} \mathrm{H}$ NMR S29 Compound $23{ }^{13} \mathrm{C}$ NMR S30 Compound $24{ }^{1} \mathrm{H}$ NMR S31

Compound $24{ }^{13} \mathrm{C}$ NMR S32 Compound $25{ }^{1} \mathrm{H}$ NMR S33 Compound $25{ }^{13} \mathrm{C}$ NMR S34 Compound $26{ }^{1} \mathrm{H}$ NMR 
Table of Contents, cont.

$\begin{array}{ll}\text { Compound } 26{ }^{13} \mathrm{C} \text { NMR } & \text { S37 }\end{array}$

Compound $27^{1} \mathrm{H}$ NMR $\quad$ S38

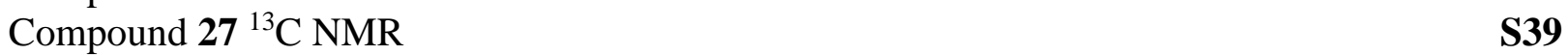

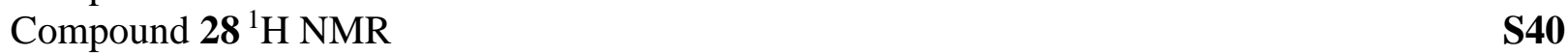

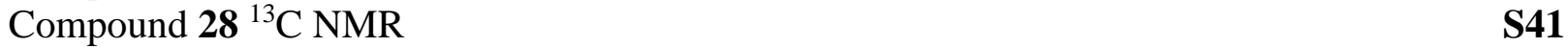

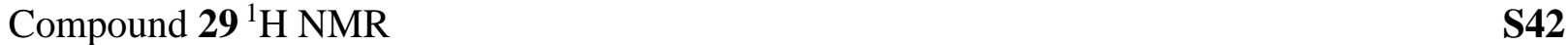

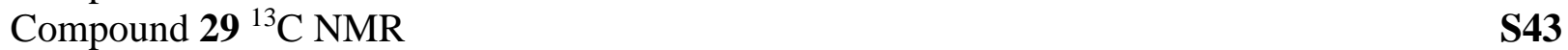

Compound $30^{1} \mathrm{H}$ NMR $\quad$ S44

Compound $30{ }^{13} \mathrm{C}$ NMR $\quad$ S45

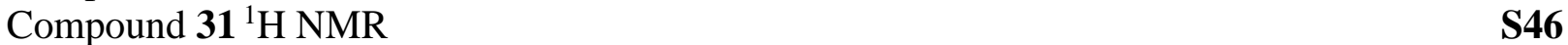

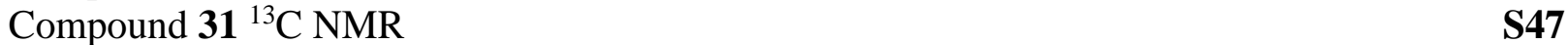

$\begin{array}{ll}\text { Compound } 32{ }^{1} \mathrm{H} \text { NMR } & \mathbf{S 4 8}\end{array}$

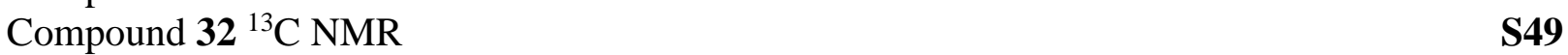

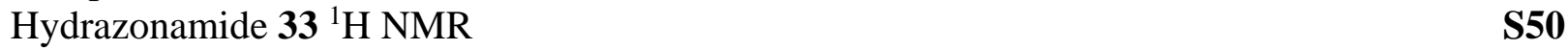

Hydrazonamide $33^{13} \mathrm{C}$ NMR $\quad$ S51

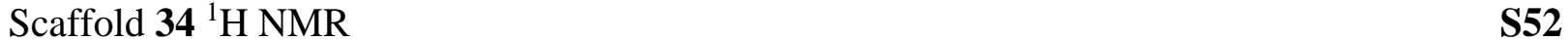

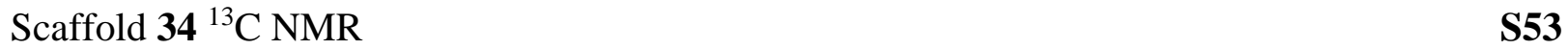

Scaffold $35{ }^{1} \mathrm{H}$ NMR $\quad$ S54

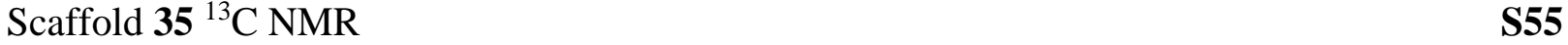

Scaffold $36{ }^{1} \mathrm{H}$ NMR $\quad$ S56

Scaffold $36{ }^{13} \mathrm{C}$ NMR $\quad$ S57

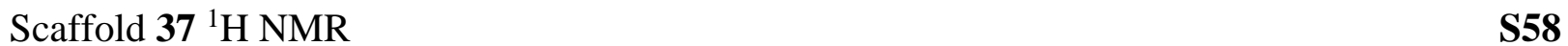

Scaffold $37{ }^{13} \mathrm{C}$ NMR $\quad$ S59

Chromatogram of $\mathbf{6} \quad \mathbf{S 6 0}$

Chromatogram of $\mathbf{1 1} \quad$ S61

Chromatogram of $12 \quad$ S62

$\begin{array}{ll}\text { Chromatogram of } 13 & \text { S63 }\end{array}$

Chromatogram of $14 \quad$ S64

Chromatogram of $15 \quad S 65$

Chromatogram of $\mathbf{1 6} \quad$ S66

$\begin{array}{ll}\text { Chromatogram of } \mathbf{1 7} & \text { S67 }\end{array}$

$\begin{array}{lr}\text { Chromatogram of } 18 & \text { S68 }\end{array}$

Chromatogram of $19 \quad$ S69

$\begin{array}{ll}\text { Chromatogram of } \mathbf{2 0} & \text { S70 }\end{array}$

$\begin{array}{ll}\text { Chromatogram of } \mathbf{2 1} & \text { S71 }\end{array}$

$\begin{array}{ll}\text { Chromatogram of } 22 & \text { S72 }\end{array}$

Chromatogram of $23 \quad$ S73

Chromatogram of $24 \quad 574$

Chromatogram of $25 \quad S 75$

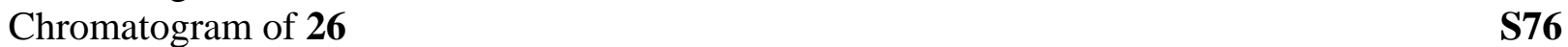

$\begin{array}{lr}\text { Chromatogram of } 27 & \text { S77 }\end{array}$

$\begin{array}{ll}\text { Chromatogram of } \mathbf{2 8} & \text { S78 }\end{array}$

Chromatogram of $29 \quad$ S79

Chromatogram of $\mathbf{3 0} \quad \mathbf{S 8 0}$

$\begin{array}{ll}\text { Chromatogram of } \mathbf{3 1} & \mathbf{S 8 1}\end{array}$ 
Table of Contents, cont.

Chromatogram of 32

S82

Chromatogram of $\mathbf{3 4}$

S83

Chromatogram of $\mathbf{3 5}$

S84

Chromatogram of $\mathbf{3 6}$

S85

Chromatogram of $\mathbf{3 7}$

S86 

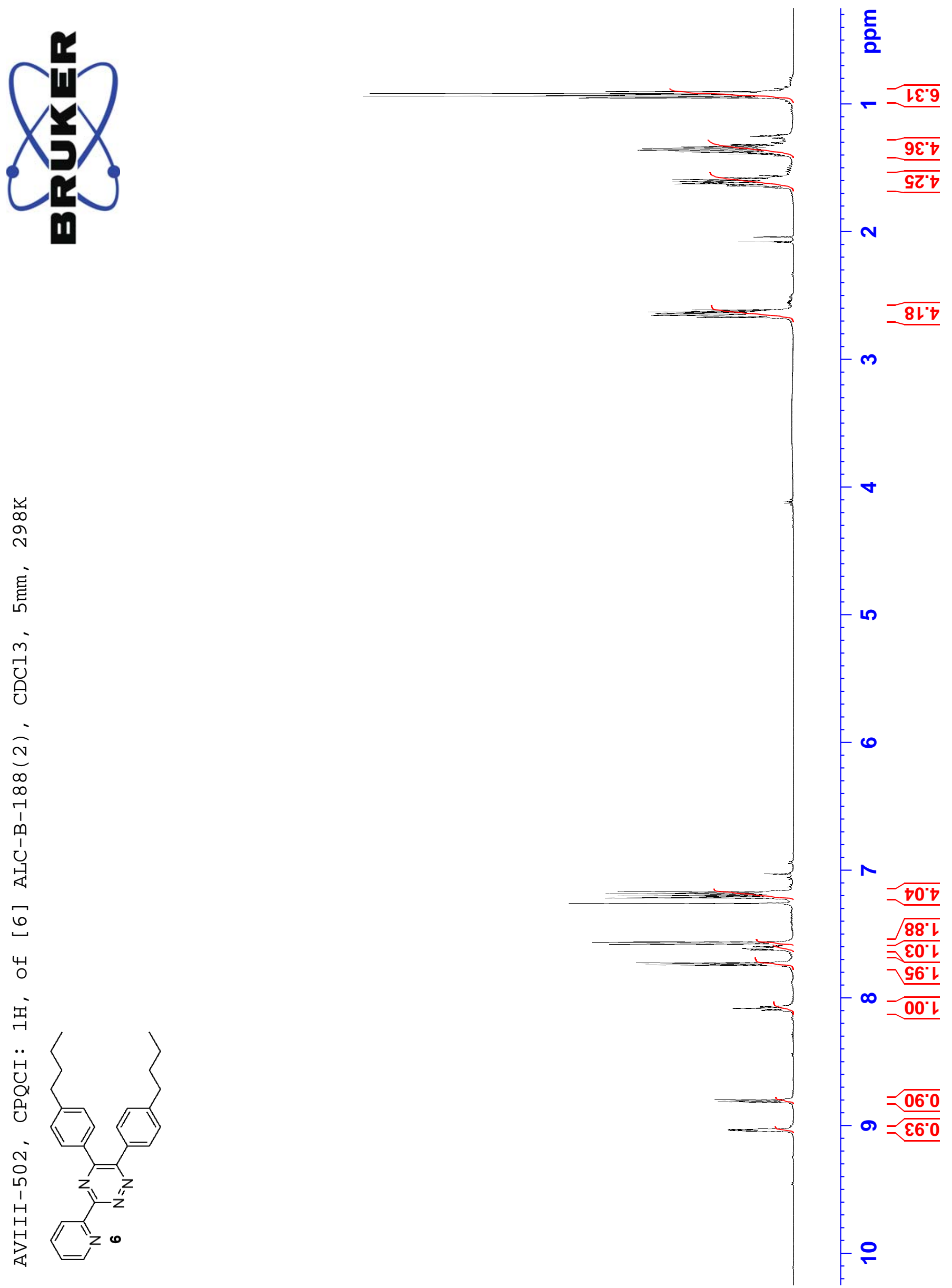


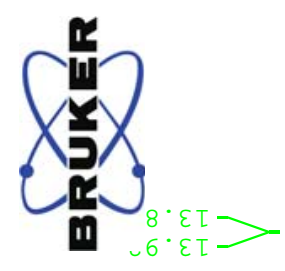

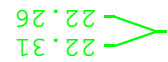

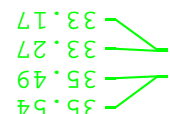

$\nabla \mathrm{G} \cdot \mathrm{GE}-$

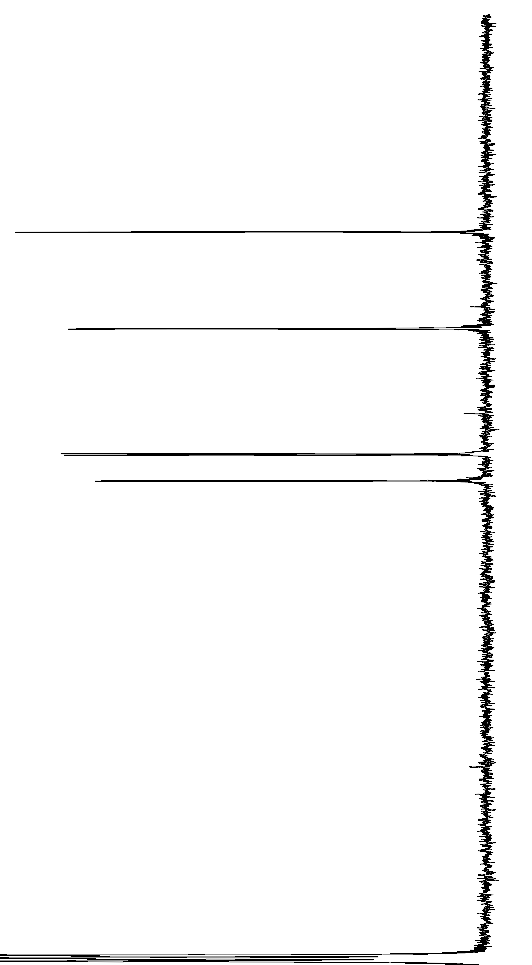

흘

우

오

요

웅

오

8

웅

용

N.

हूँ

i)

नु.

Oㄹ

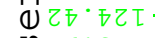

テのI6. sZT

กิ $\sigma^{69.82 T}$

$\infty$ त

$\infty$ ठ IL"

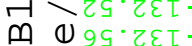

1 U

ن.

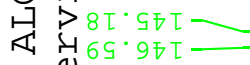

$4 \operatorname{sic}^{98.80 T}=$

०

U ن

가

감

벙응

잉

-iv

ํำ

ᄂ

동

번응

¿它
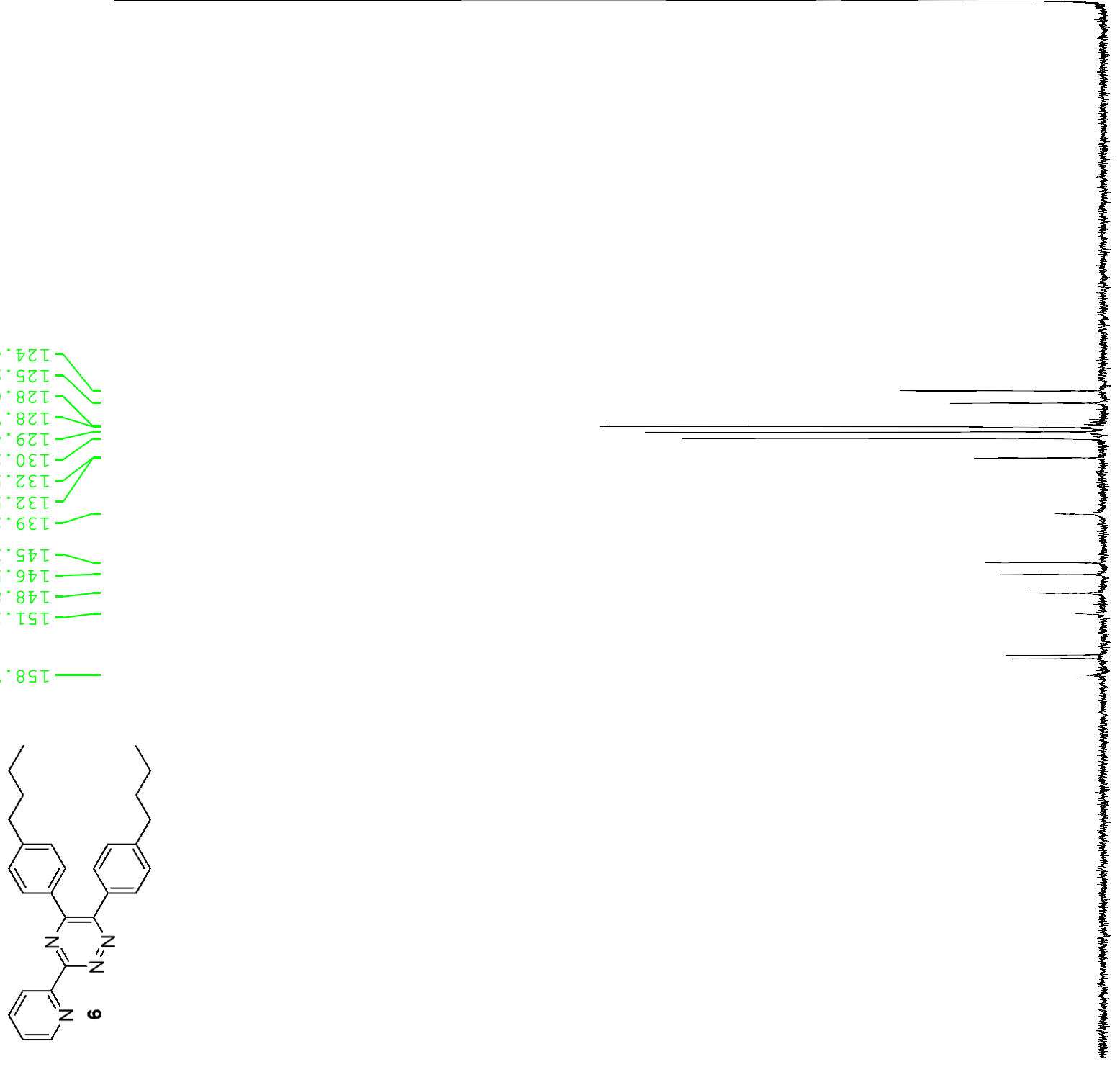

$\infty$

8

용

옥

กิ

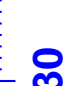

$\stackrel{m}{\longrightarrow}$

욱

욱

8

옥

$\stackrel{\circ}{-}$

음

=유 


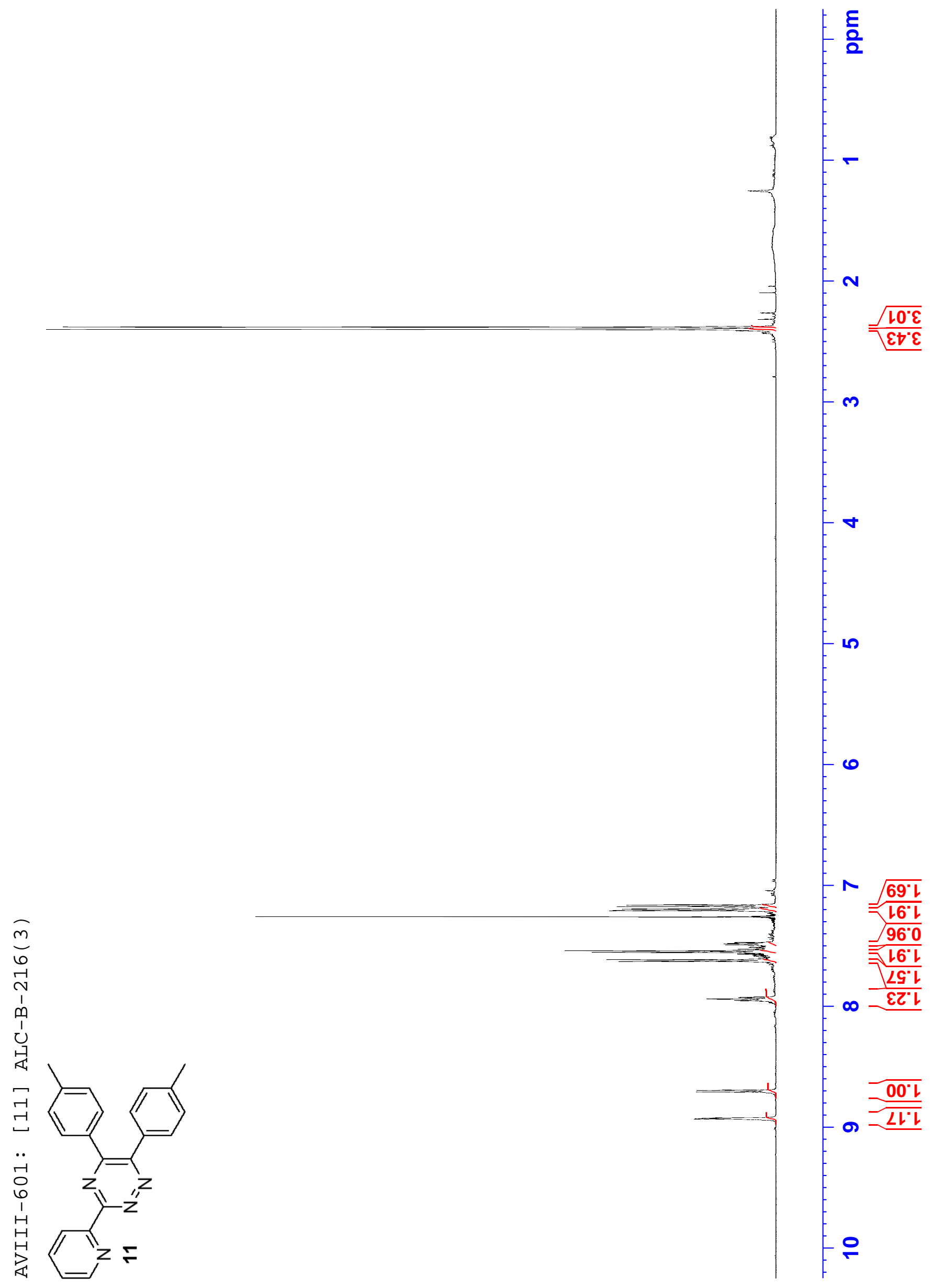



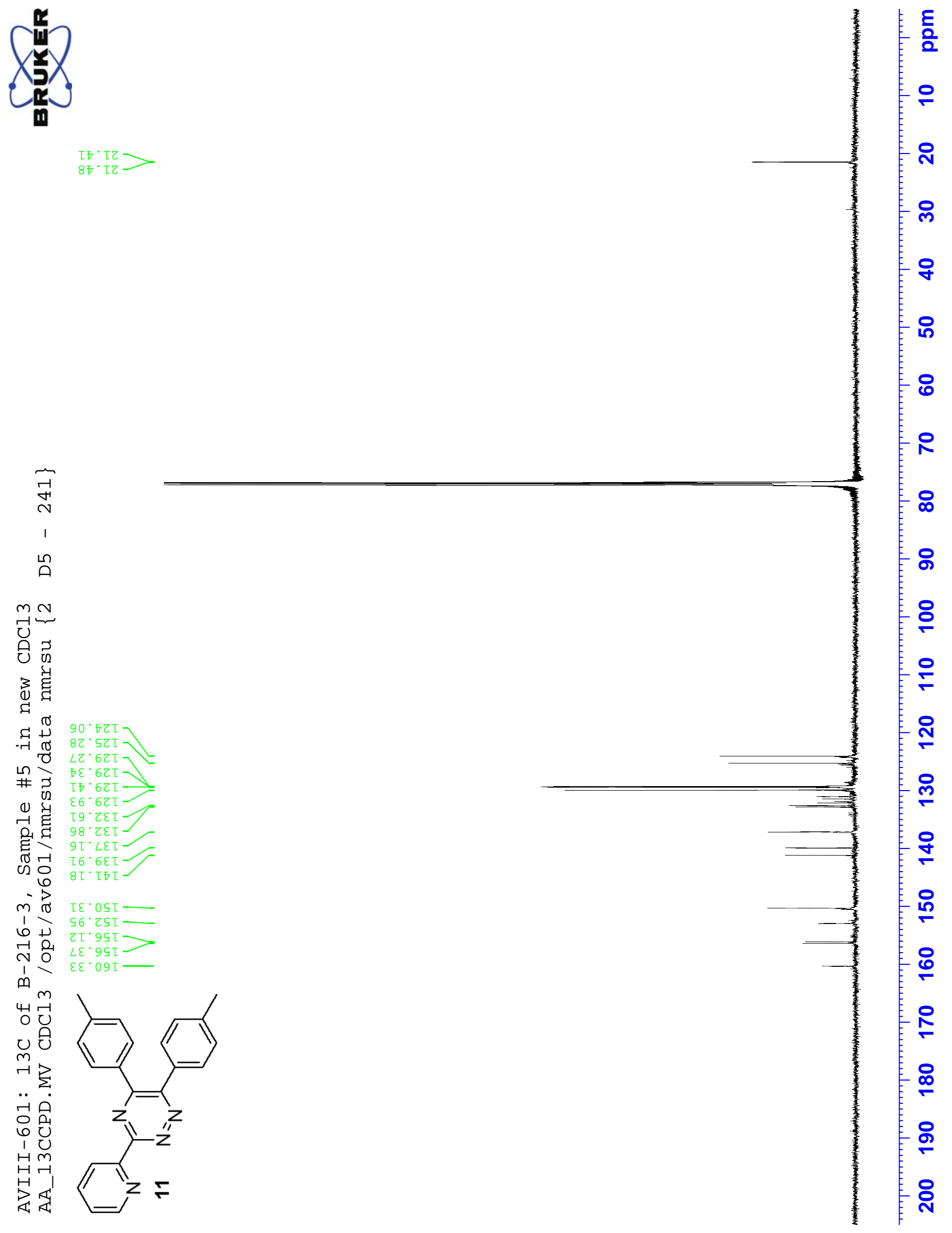


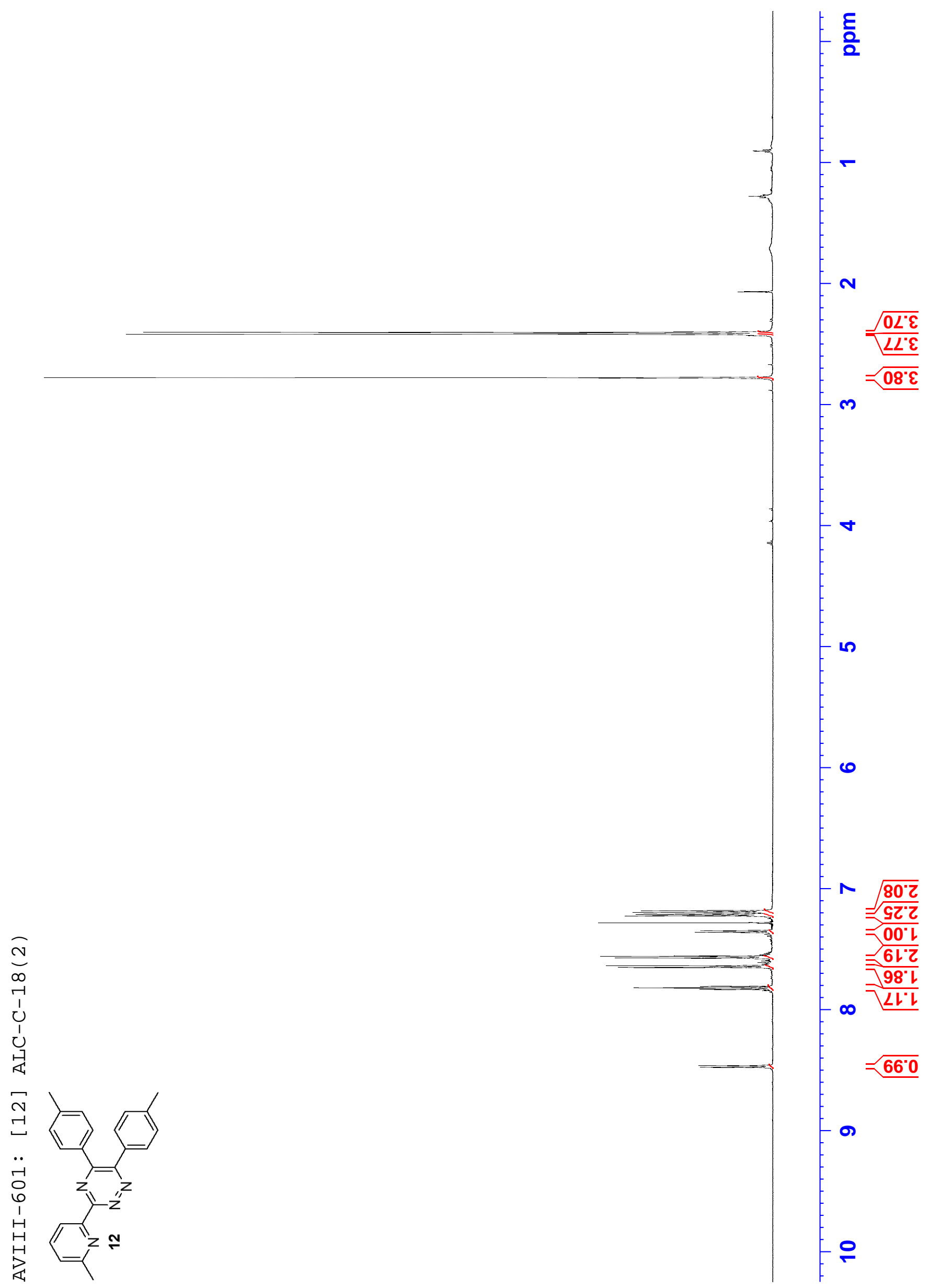


㩆

It"IZ

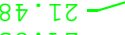

ง8' ๖乙

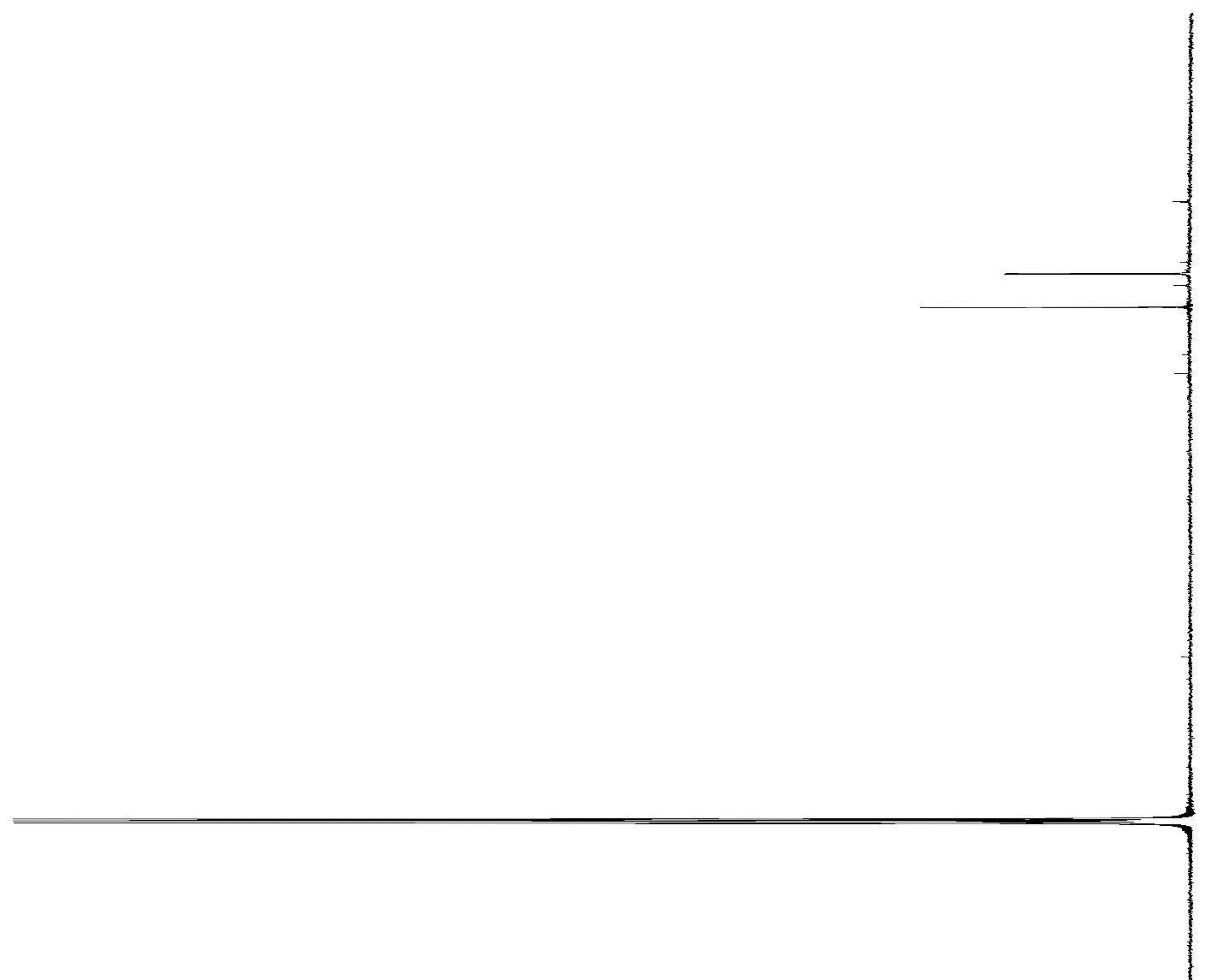

등

욱

유

요

q

요

8

$\stackrel{\substack{n \\ N}}{\sim}$

옷

$\infty$

กั

웅

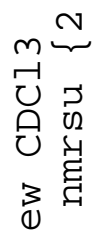

ฮ。

ᄃ듀

う

\#的

9. $92 \mathrm{CL}$

$\varepsilon \cdot 62 \mathrm{~T}$

$6 \cdot 62 \mathrm{~T}$

(1) हो

L' 'ZEI

을ㄱ $60^{\circ} \angle \varepsilon \tau T$

जळ

Nं $9 g^{\circ} z 9 T$

1

8L'GST -

악응

$6 \varepsilon \cdot 69 T$

u

09 ' 99 工
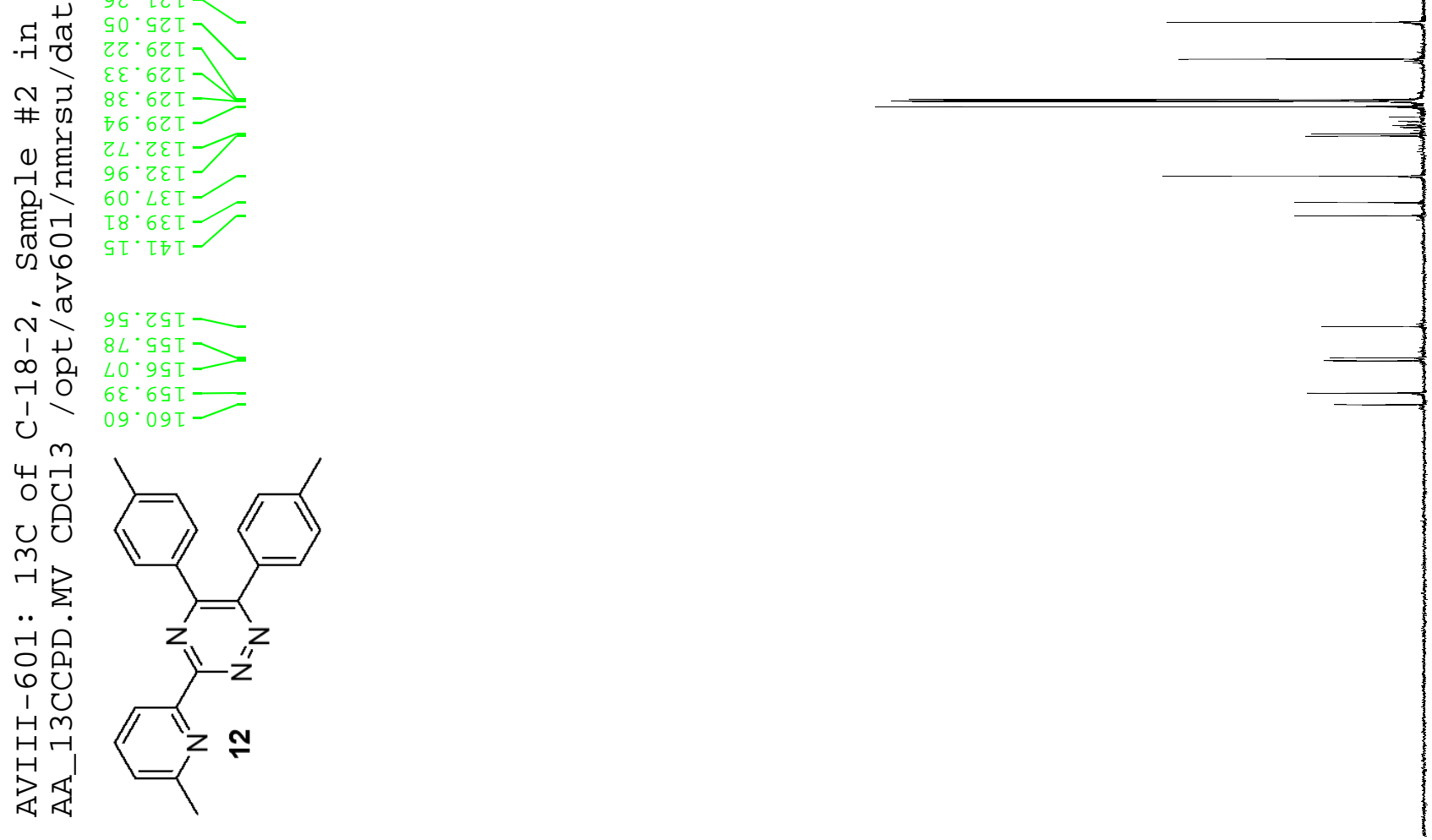

8

역

สิ

m్

年

$=$

$\stackrel{8}{0}$

只

$\stackrel{\leftrightarrow}{\circ}$

$\stackrel{8}{9}$

กั 

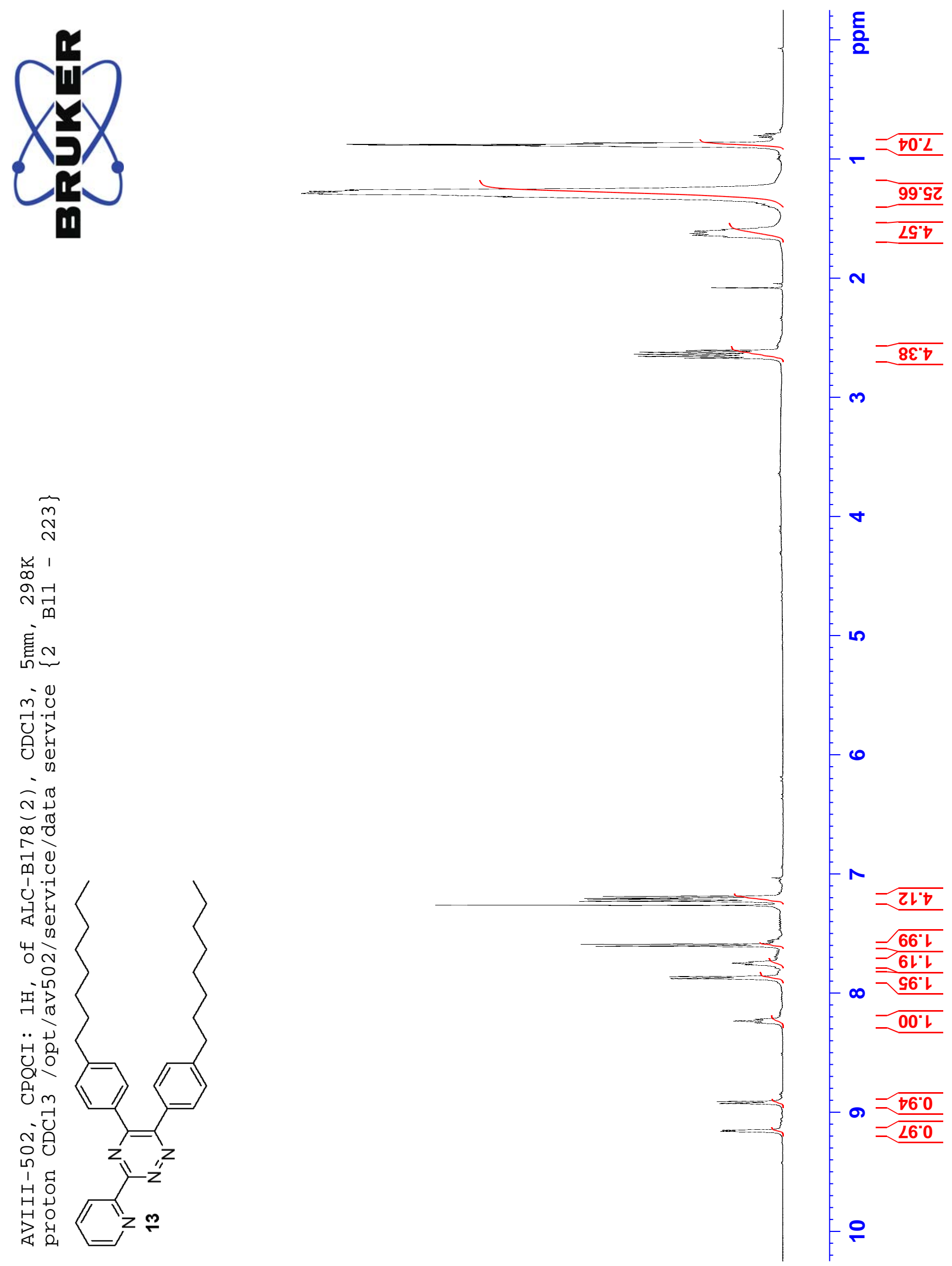


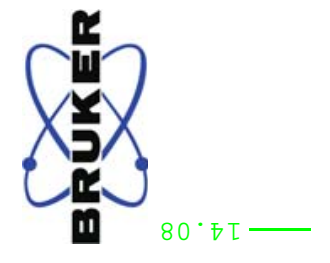

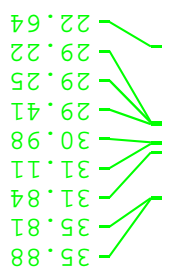

$\stackrel{\text { N }}{N}$

웜

हो

लंखे

บु

음

ते

$\infty \geq$ ot.6zI

त्र

' St'zeI

บ

$\varangle$

4 त

○े

니응

m-

m

$\because 7$

넝

U

구

๑응

10

Hल

답

\&文

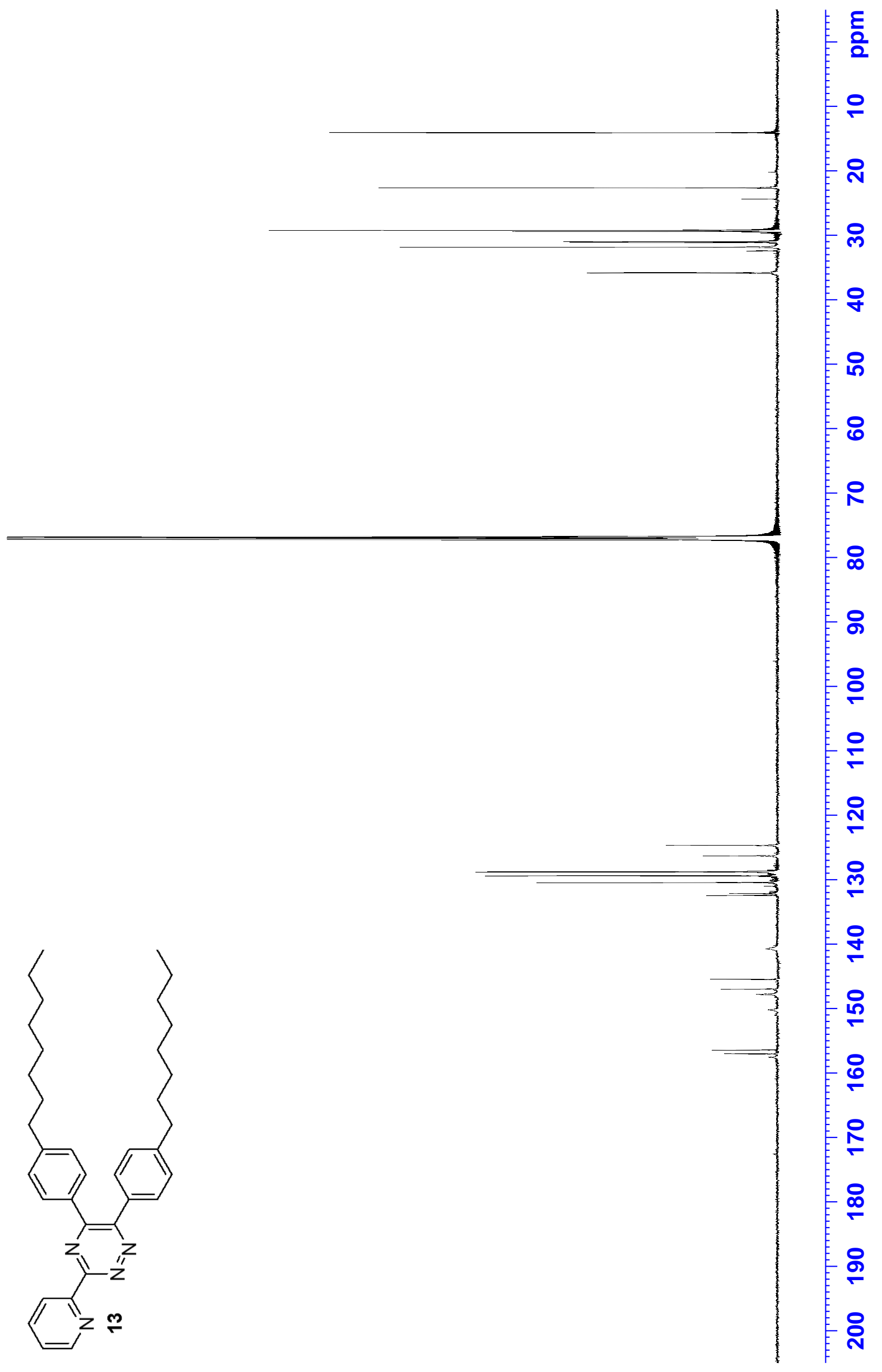



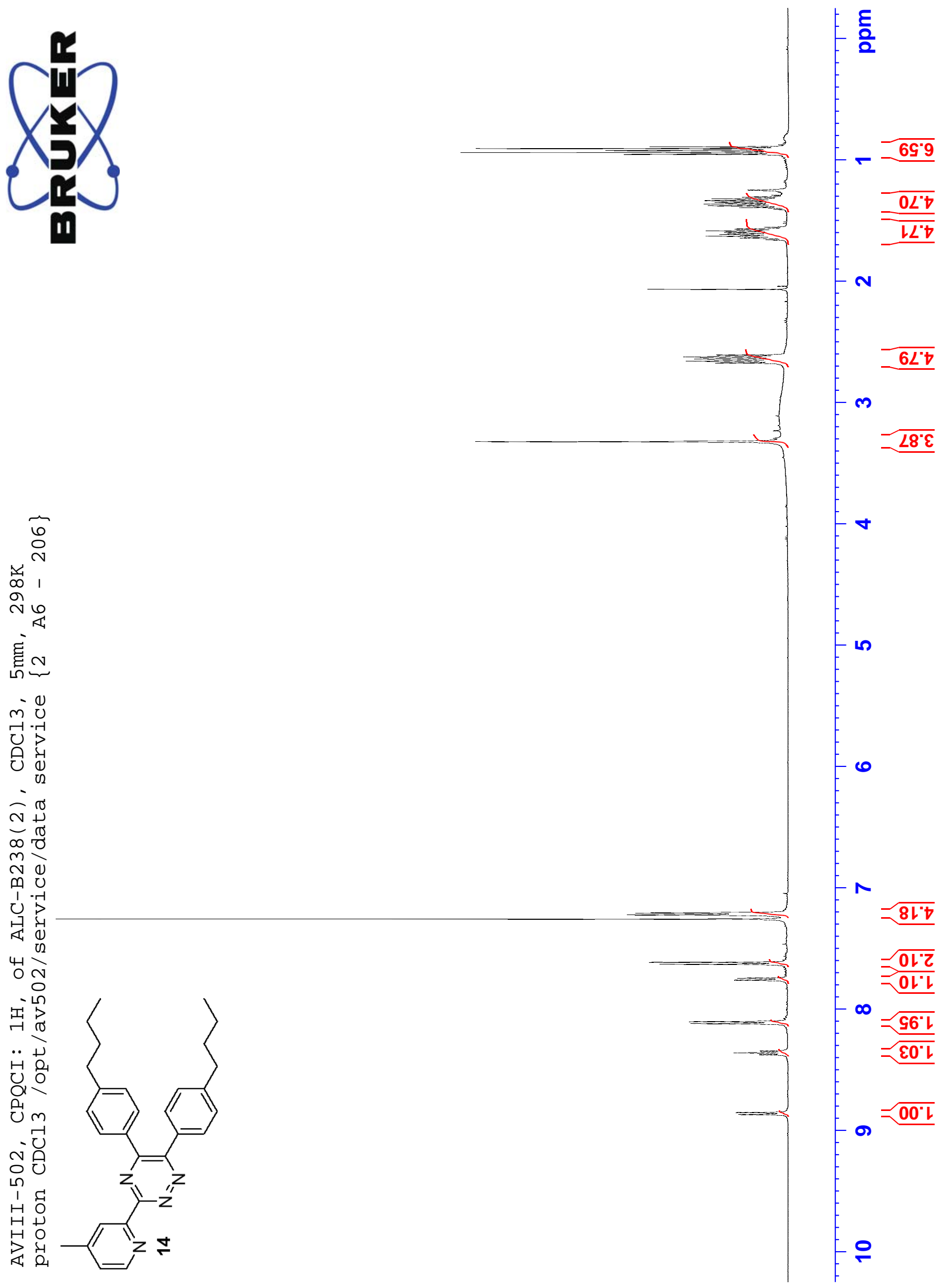
㩆

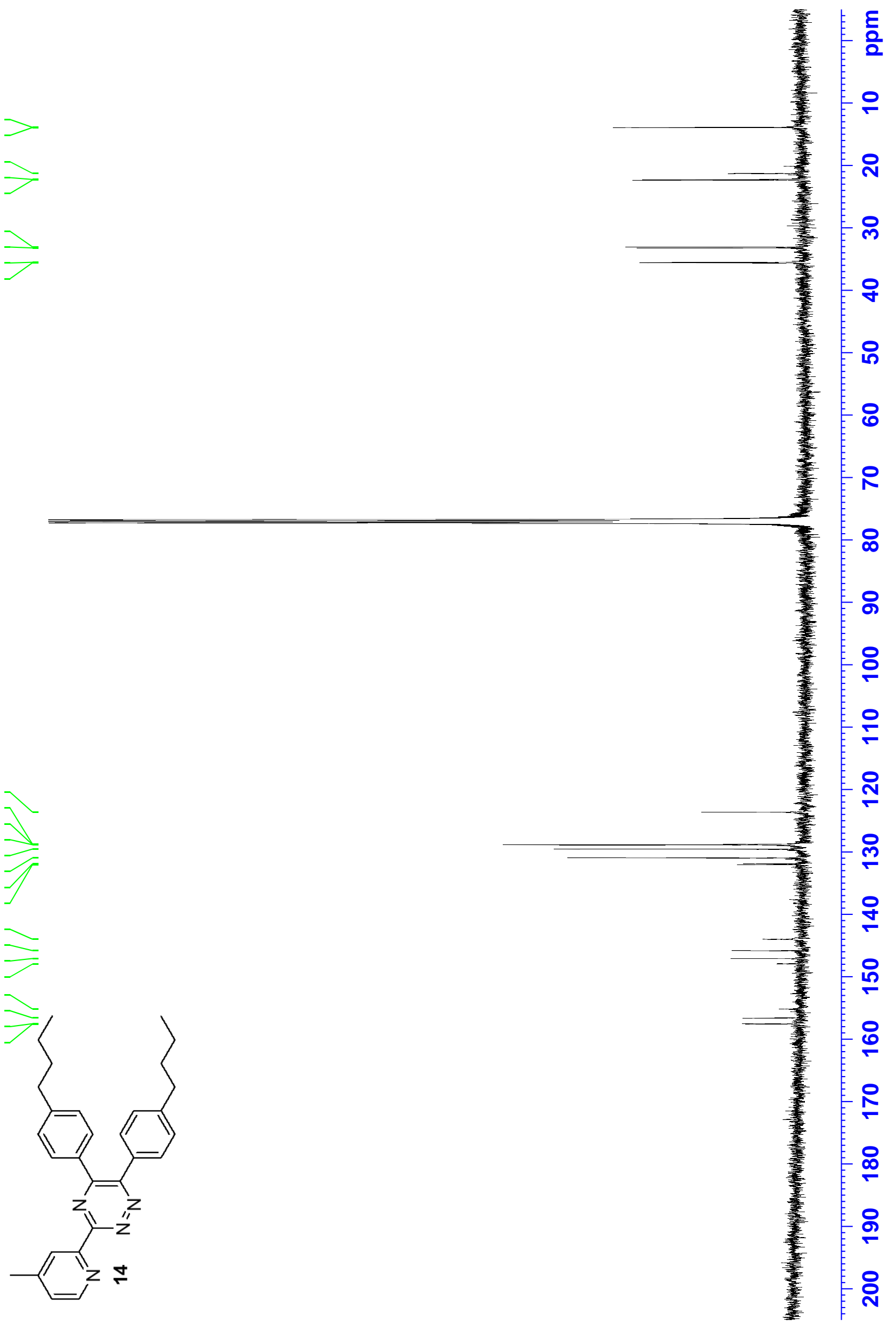

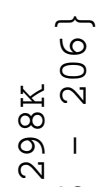

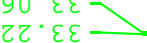

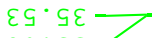

09 "

है

लं 1

넌

ㄴำ

$i^{-\infty}$

$\mathcal{N}$

I9'EZT-

9L'8ZT

$\infty \frac{1}{\sigma}$

m

$98^{\circ} 82 \mathrm{Z}$

9.6ZI

๗ิ

u.

$\frac{1}{4}$

4 ज

กे

กิ

ก

긴

$\because 1 ㅇ$

넝응

บ $\mathrm{m}$

-

ํํำ

เ

동

버응

$\gg \frac{1}{2}$

$89^{\circ} \angle S T$ 

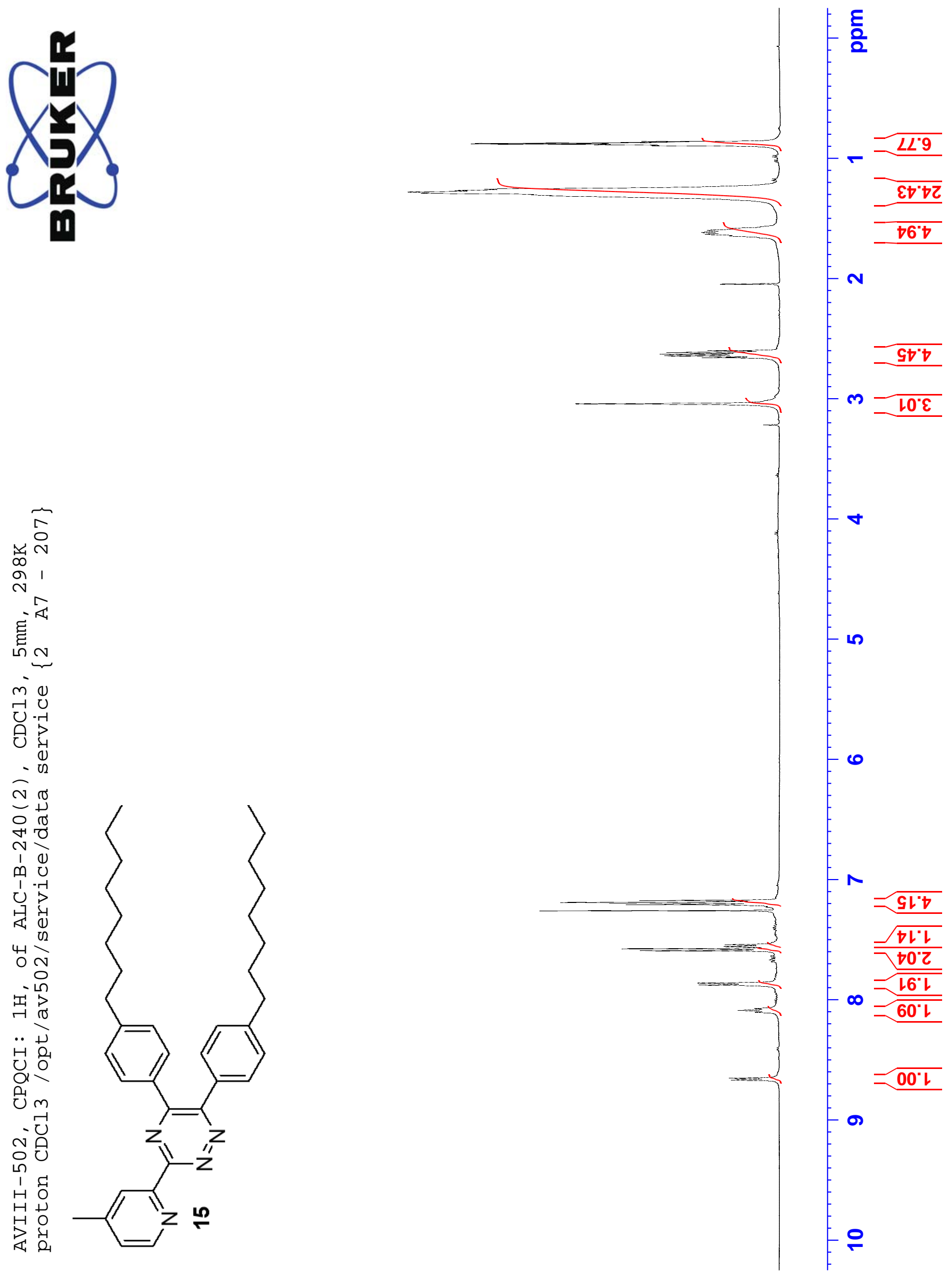

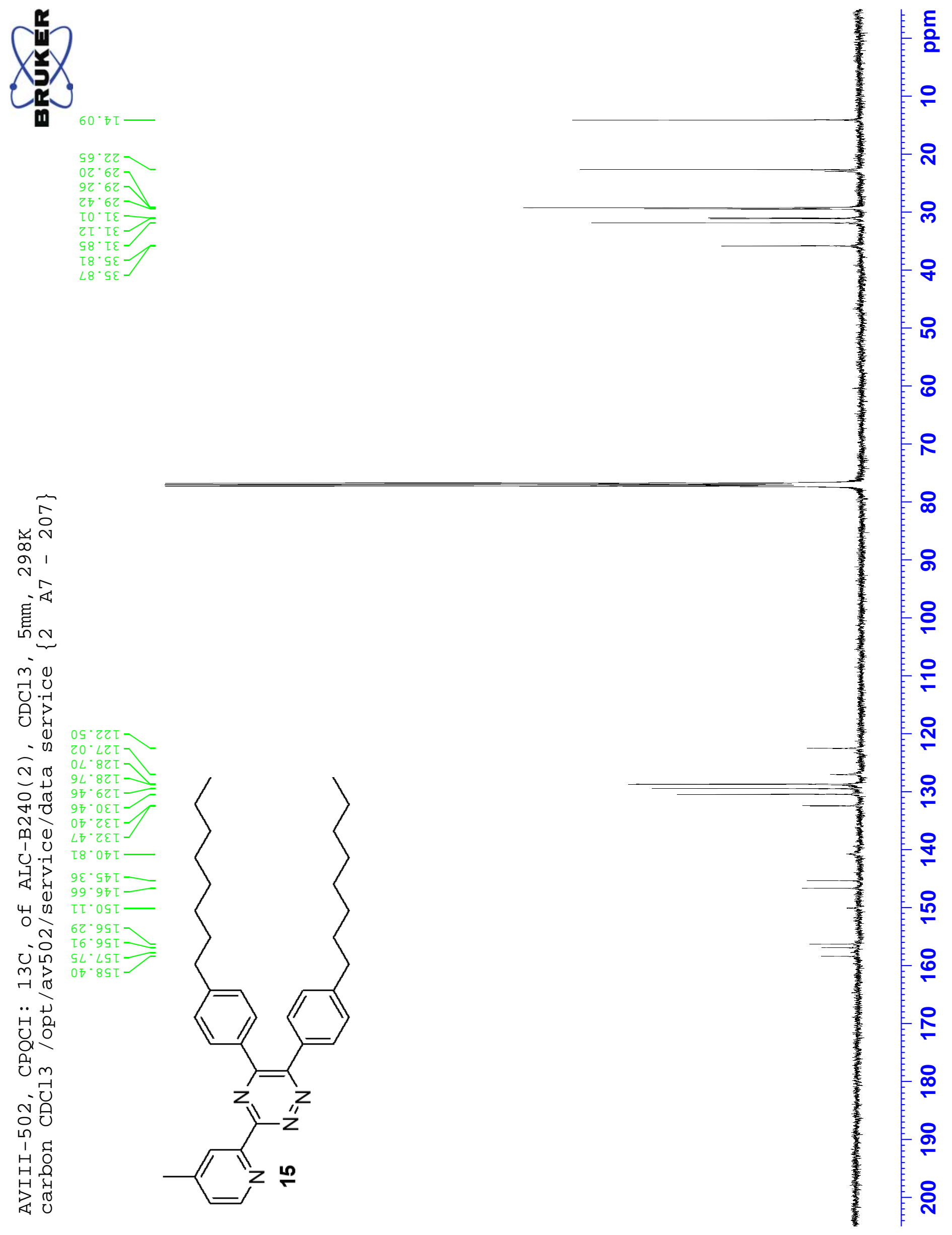

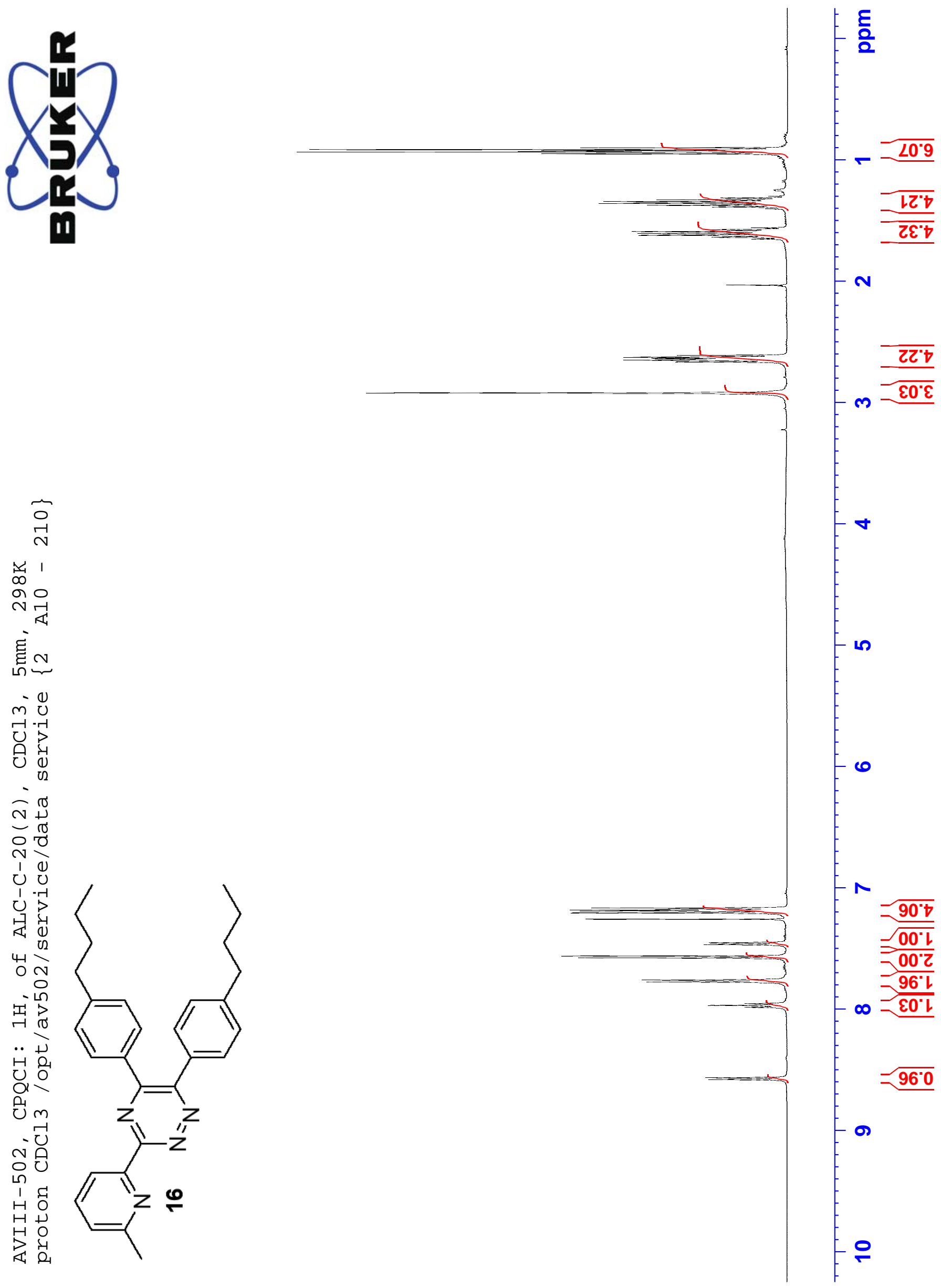

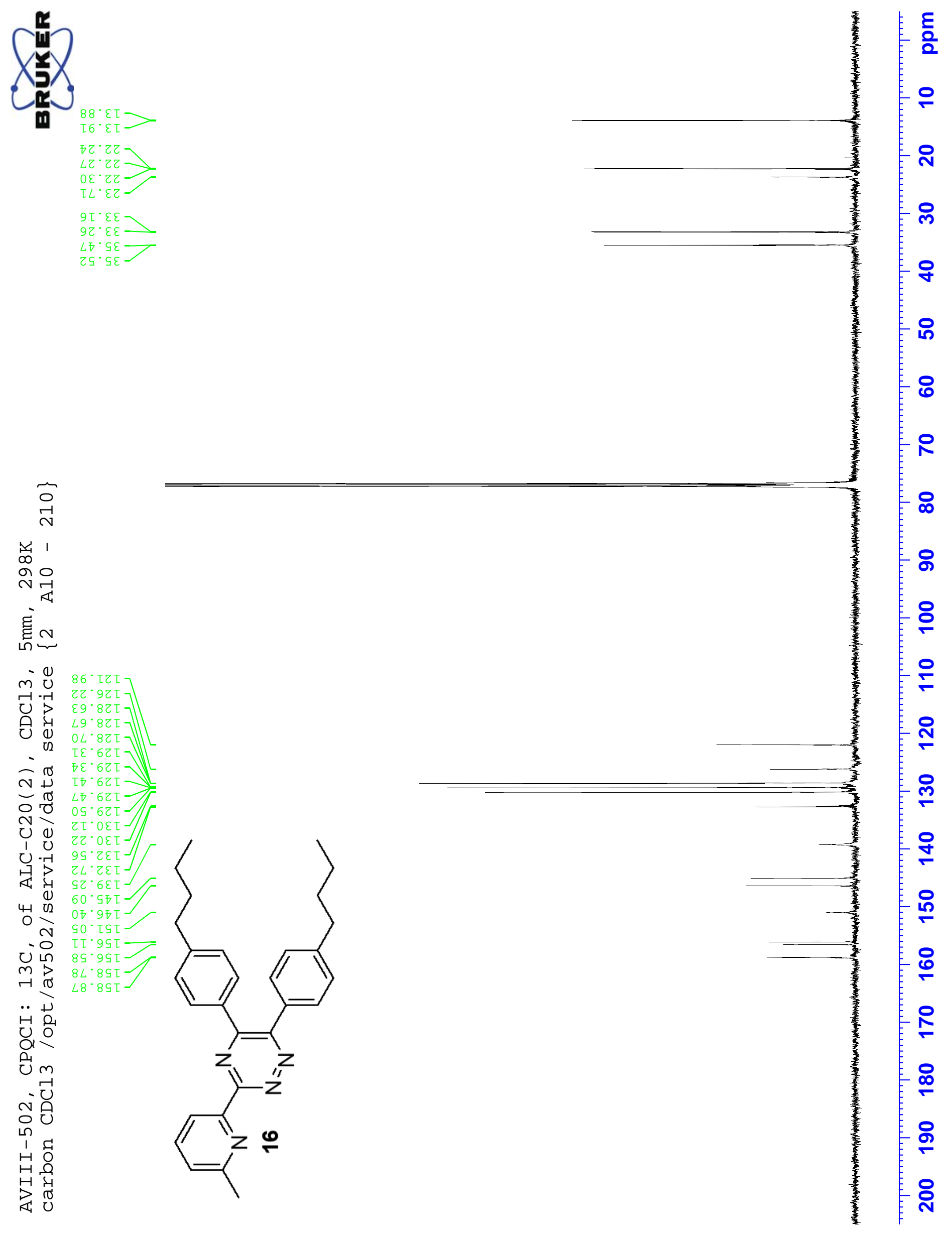

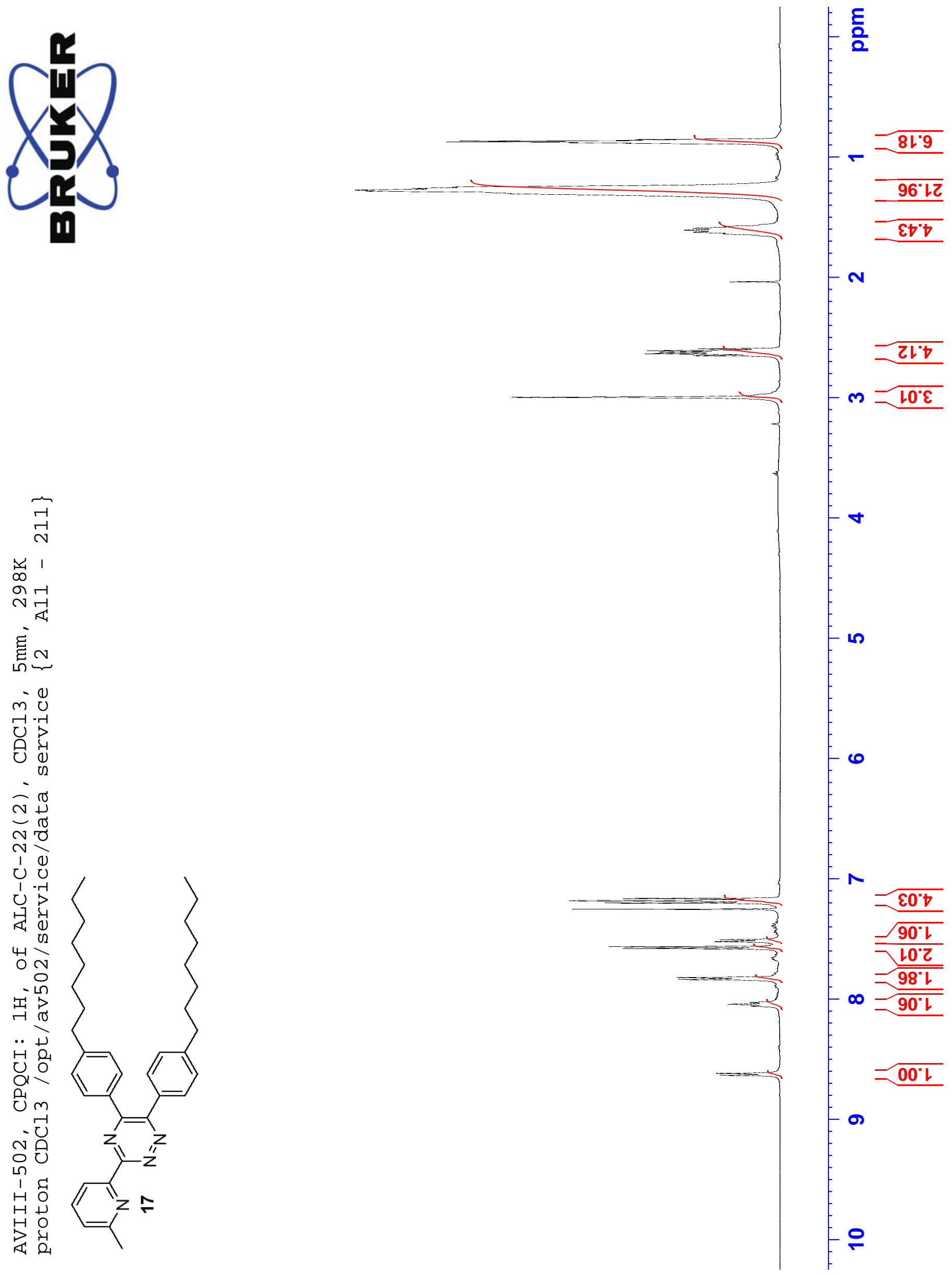

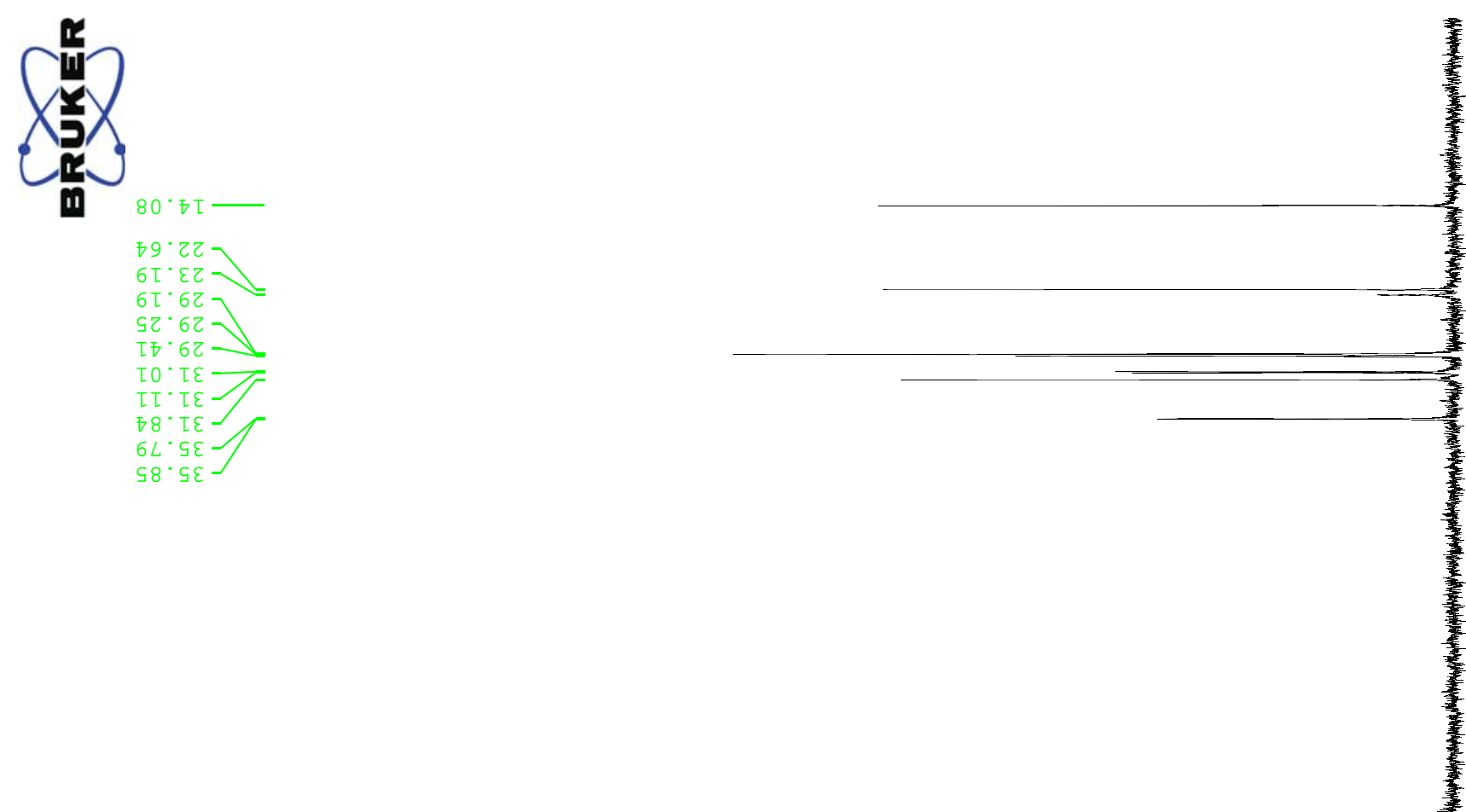

$=\frac{E}{2}$

$\triangleright 9^{\circ} \mathrm{ZZ}$

$6 \mathrm{t} \cdot 62$

$92 \cdot 62$

to.

$\triangleright 8$. $\mathrm{L} \varepsilon-$
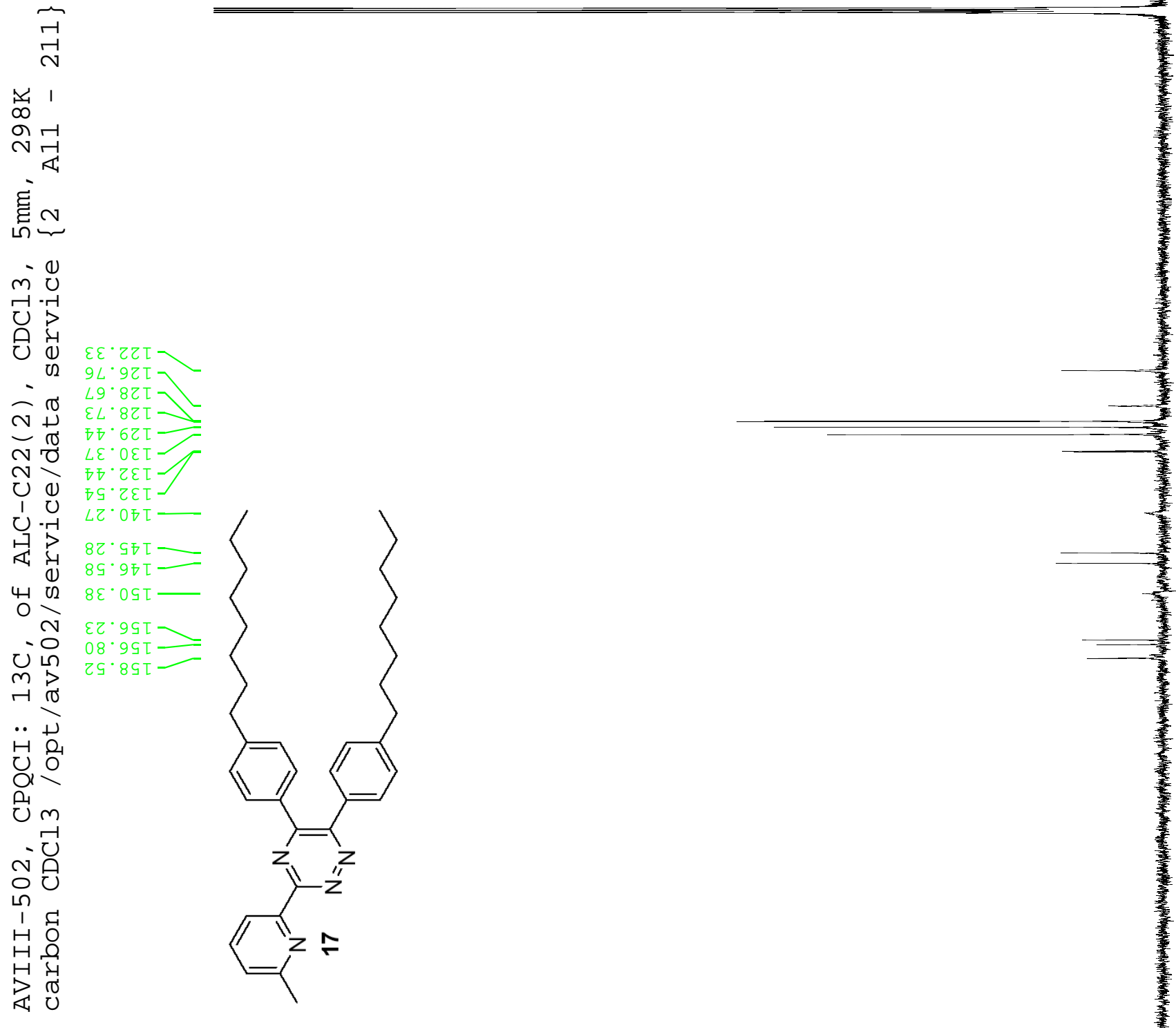

$\infty$

8

음

욱

్ㅗ

学

웍

옥

8

운

$\stackrel{\circ}{-1}$

옥

오 


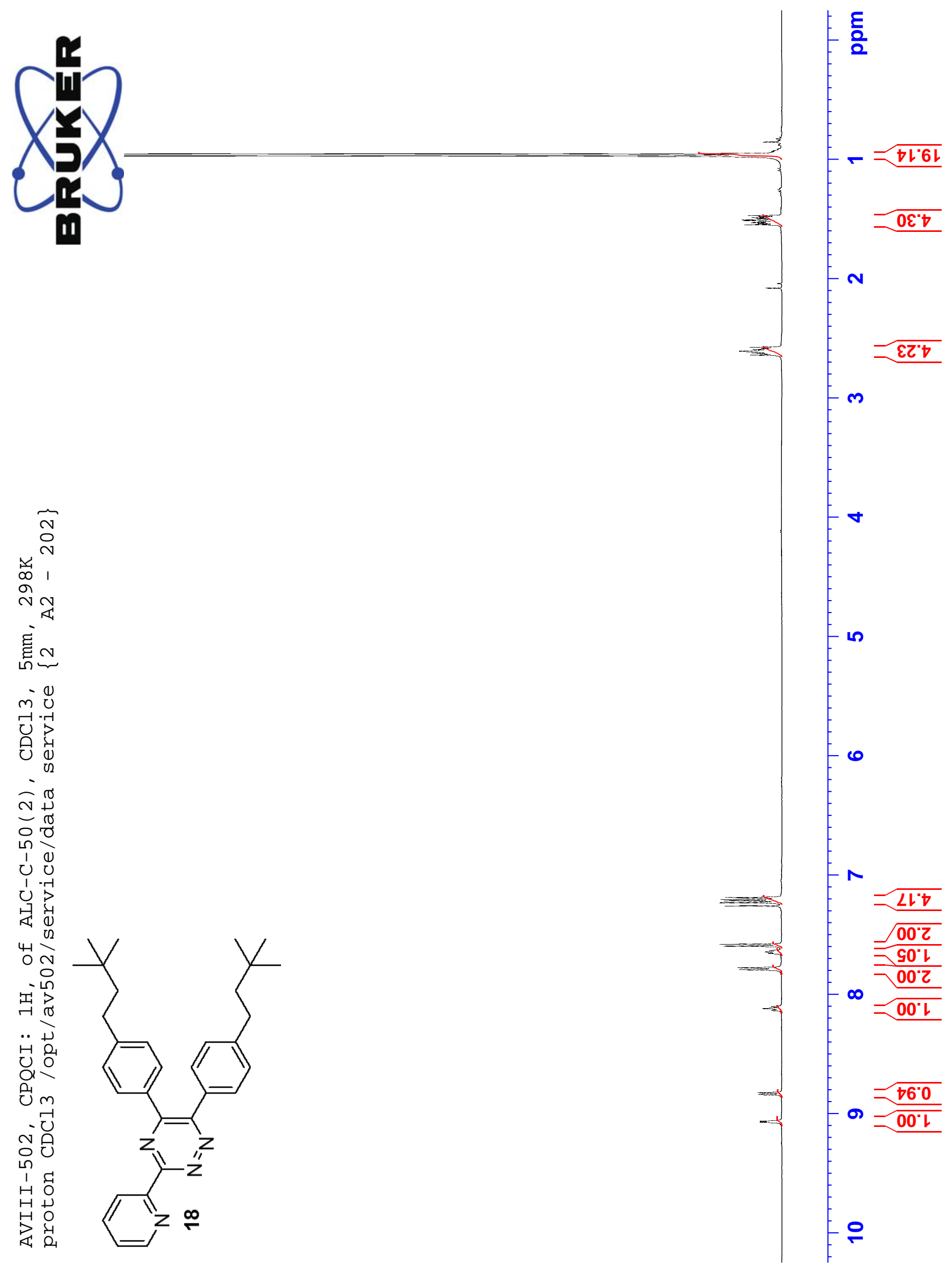



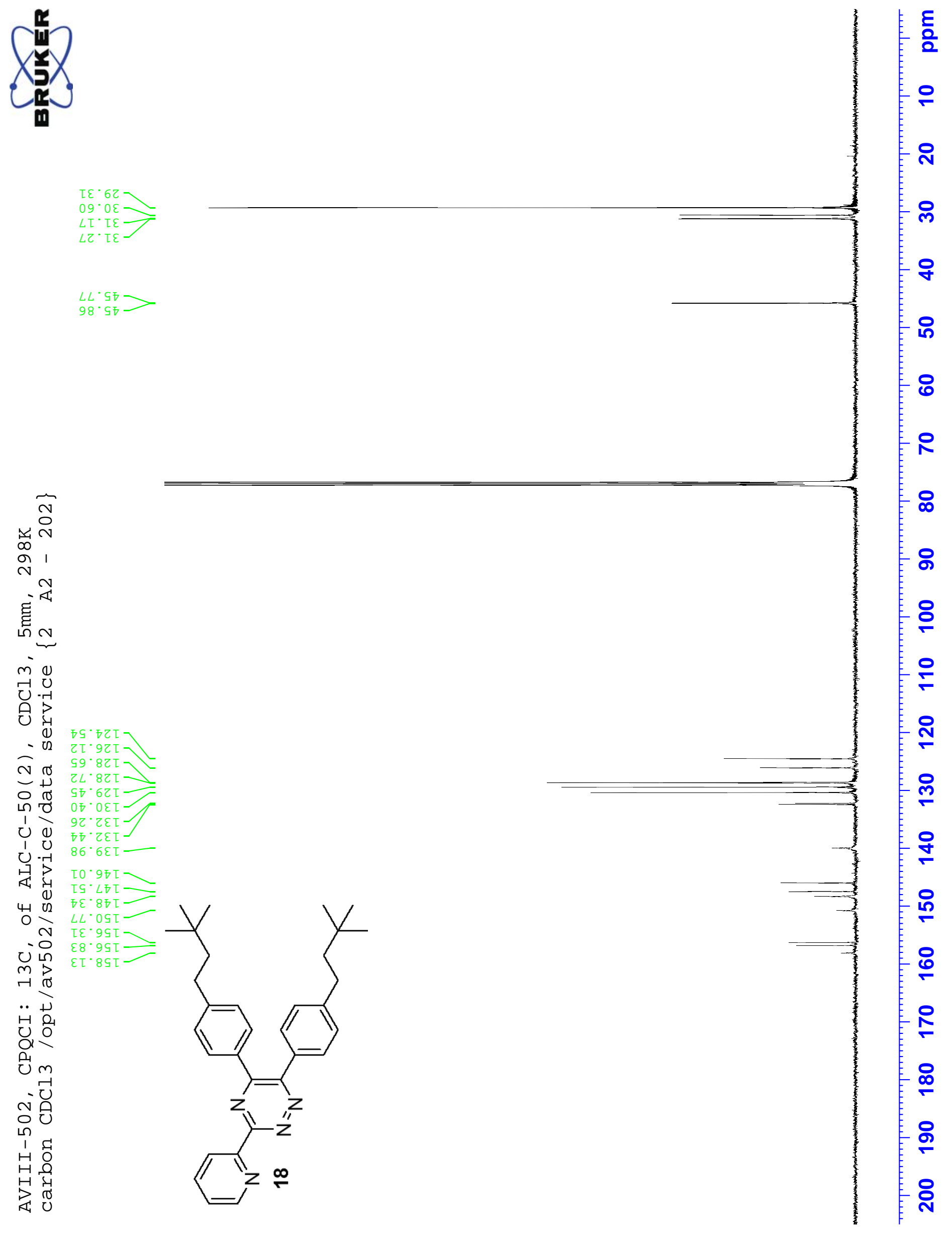


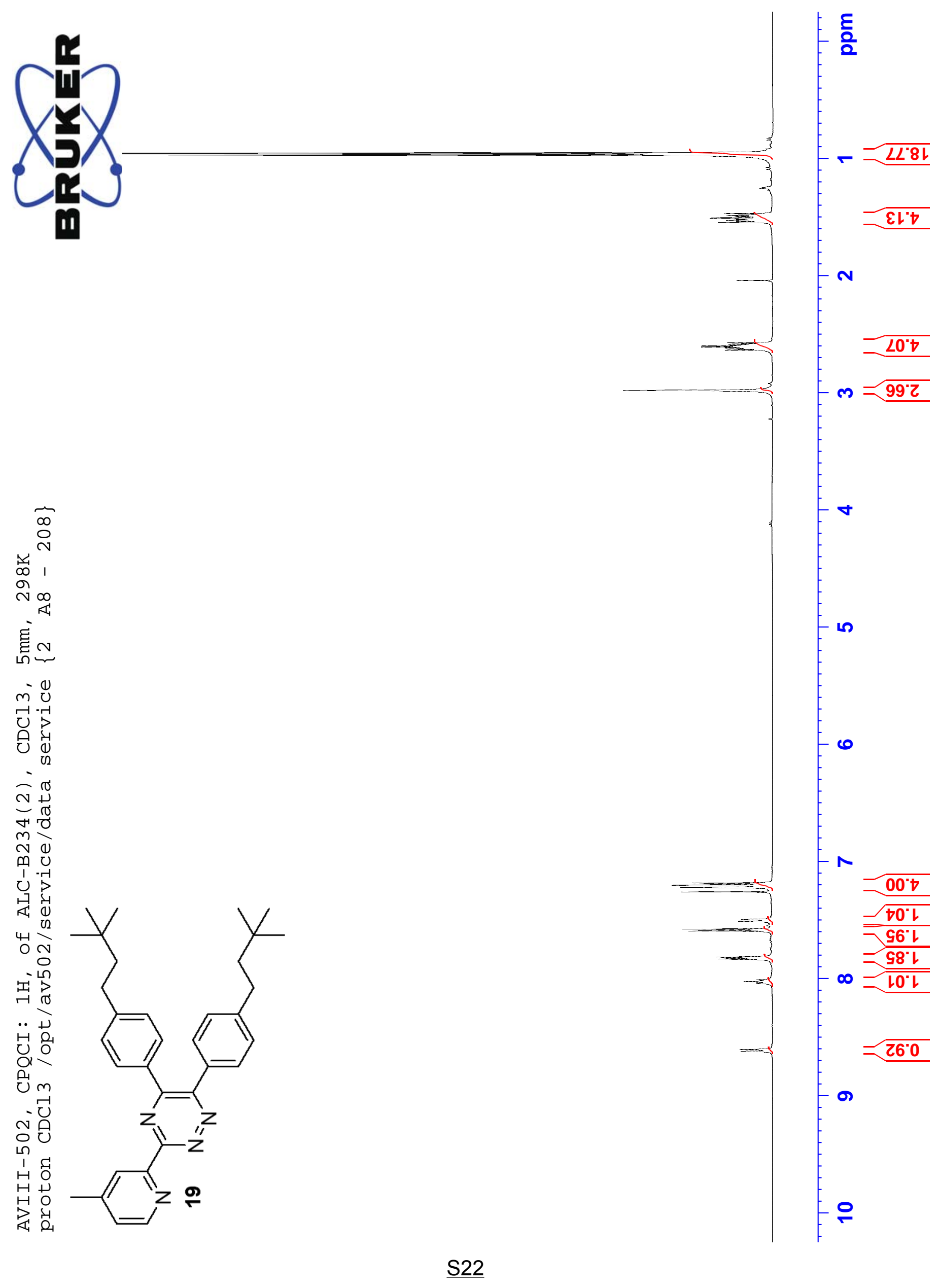


㩆

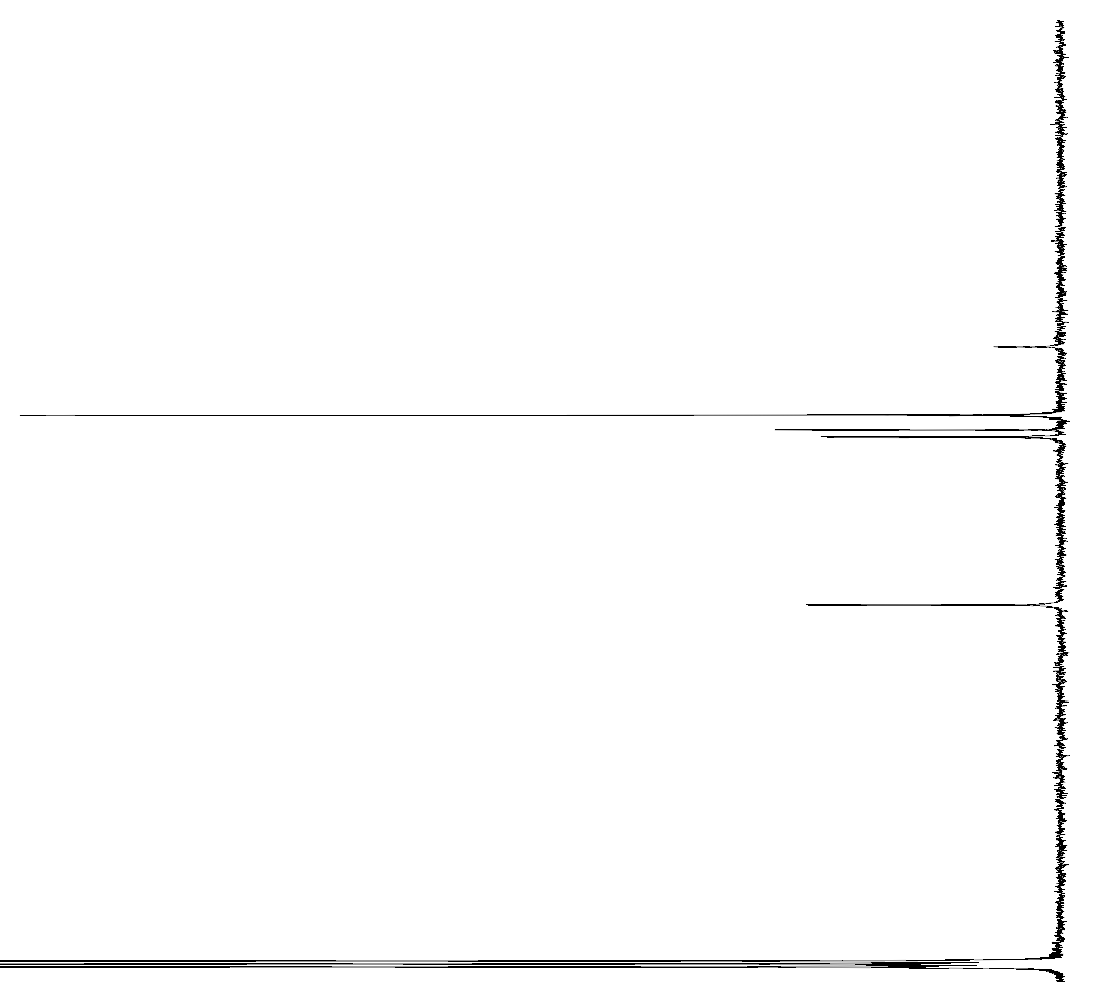

市

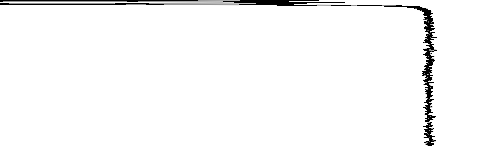

$\frac{5}{2}$

윽

오

T $\varepsilon \cdot 6 z$

$6 \mathrm{~g}^{\circ} \cdot \mathrm{- \varepsilon}$

$S I \cdot I \varepsilon>$

$\angle L \cdot S t$

98. $97>$

N

ह

लं 1

पु.

บ $\frac{1}{0}$

$\check{\sim}^{-\infty}$

N

$\underset{\sim}{+\pi}$

mo

๑ิ

ن

U. तो $\nabla g^{\circ} z \varepsilon t$

《广

ब $\nabla{ }^{\circ} \angle D T=$

पे

-ᄋ $\angle 9.997>$

ט

m

..

붕응

넝응

บ $\mathrm{m}$

단

๙ิำ

๑ั่

동

븜을

$\mapsto \frac{1}{2}$
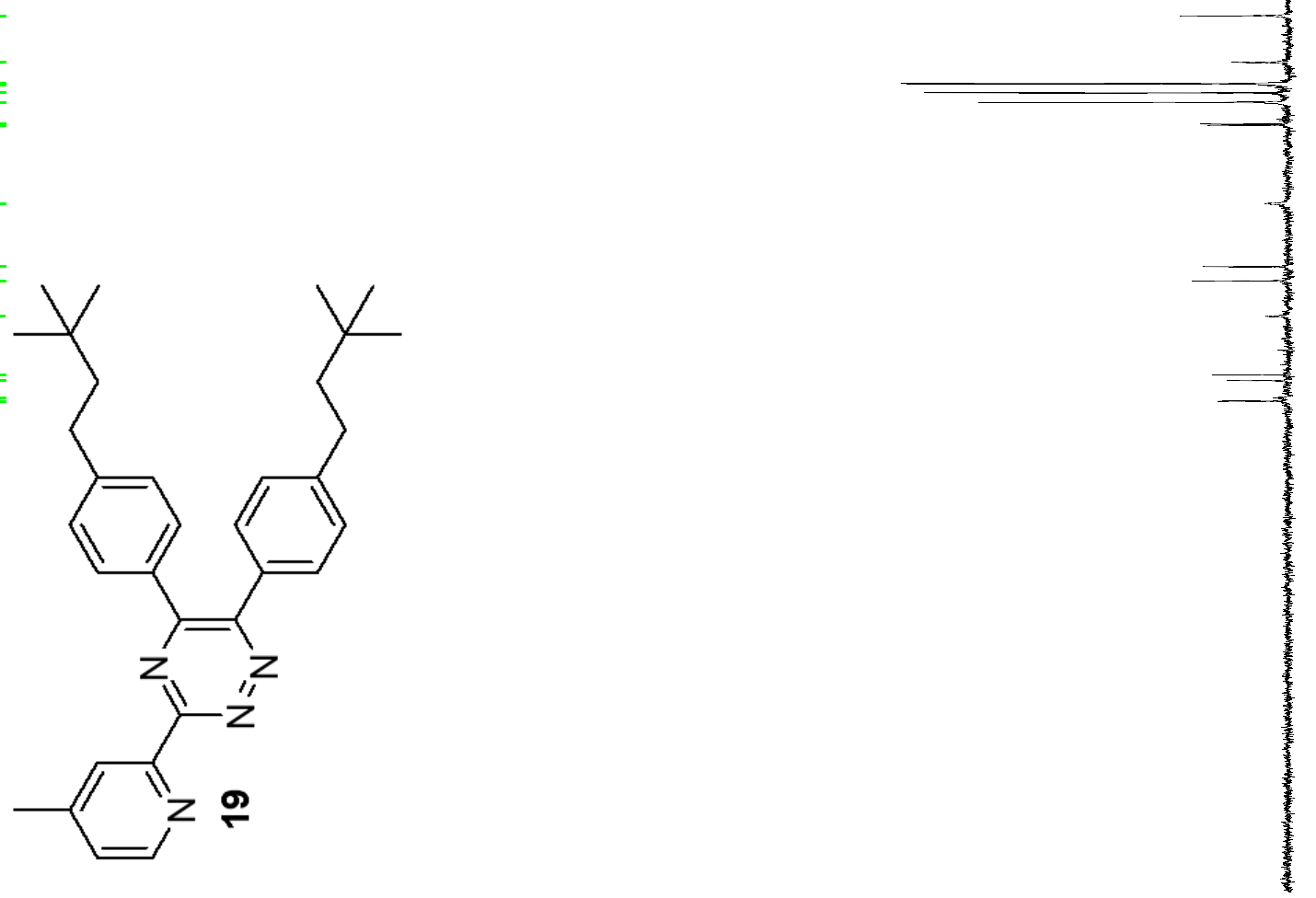

오

웅

요

8

웅

$\infty$

8

욱

옥

ํำ

学

卖

옥

$\stackrel{\circ}{\circ}$

옥

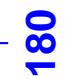

옥

ํํ 


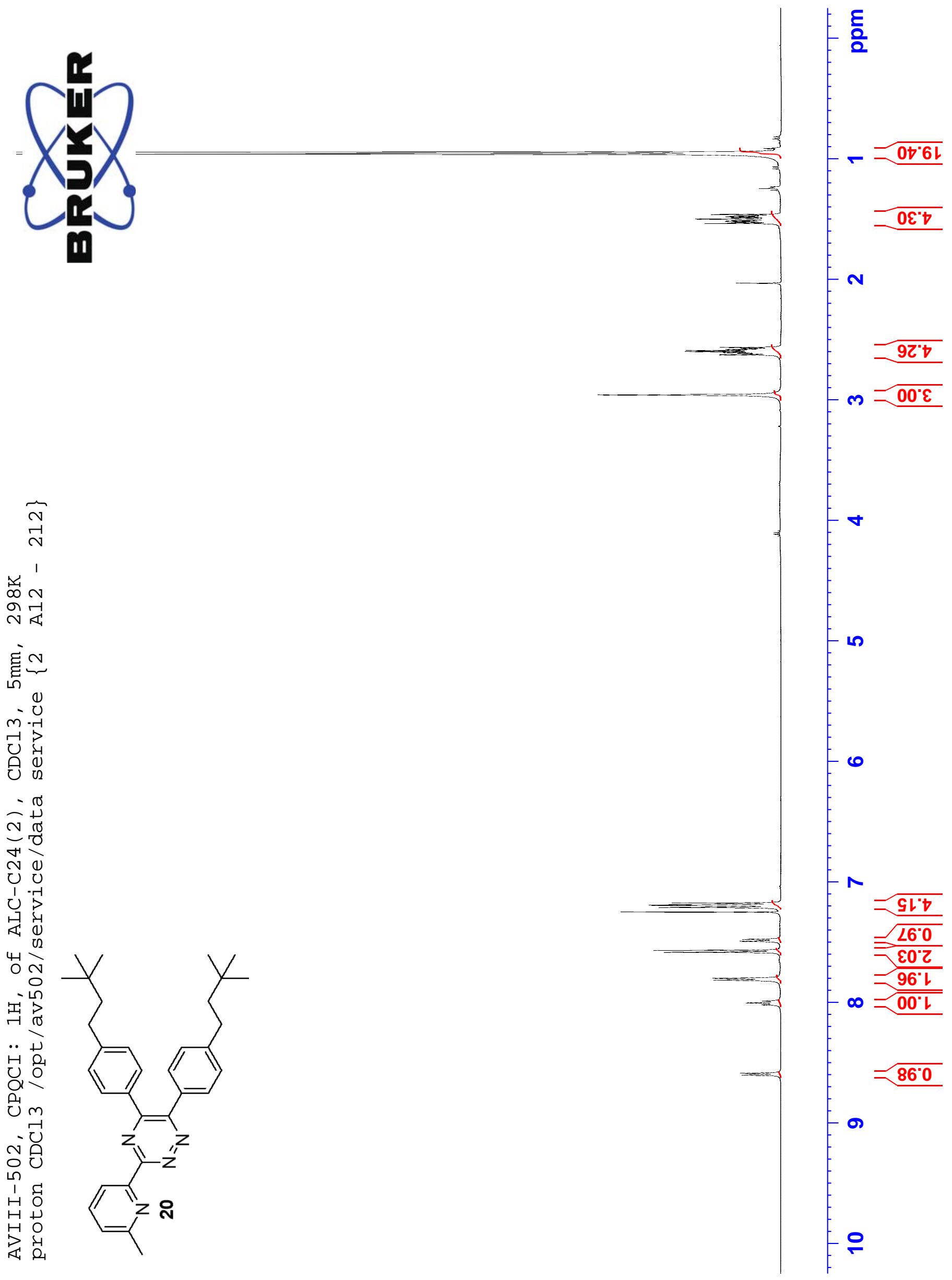


影

हू

๑७ $\varepsilon 乙$

IE' 6 乙

69 ' $0 \varepsilon$

GI" $\tau \mathcal{G} \longrightarrow$

S8. $97>$
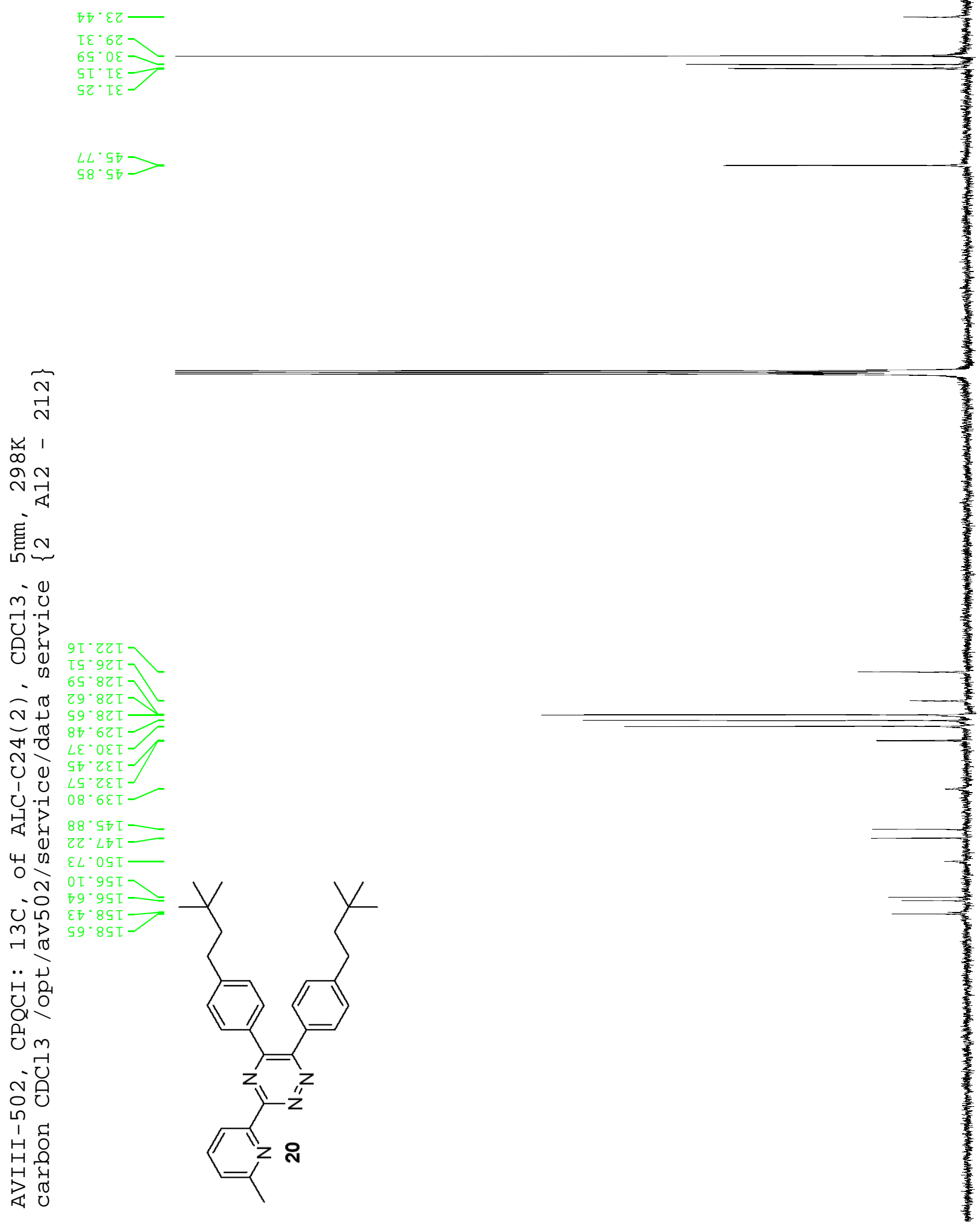

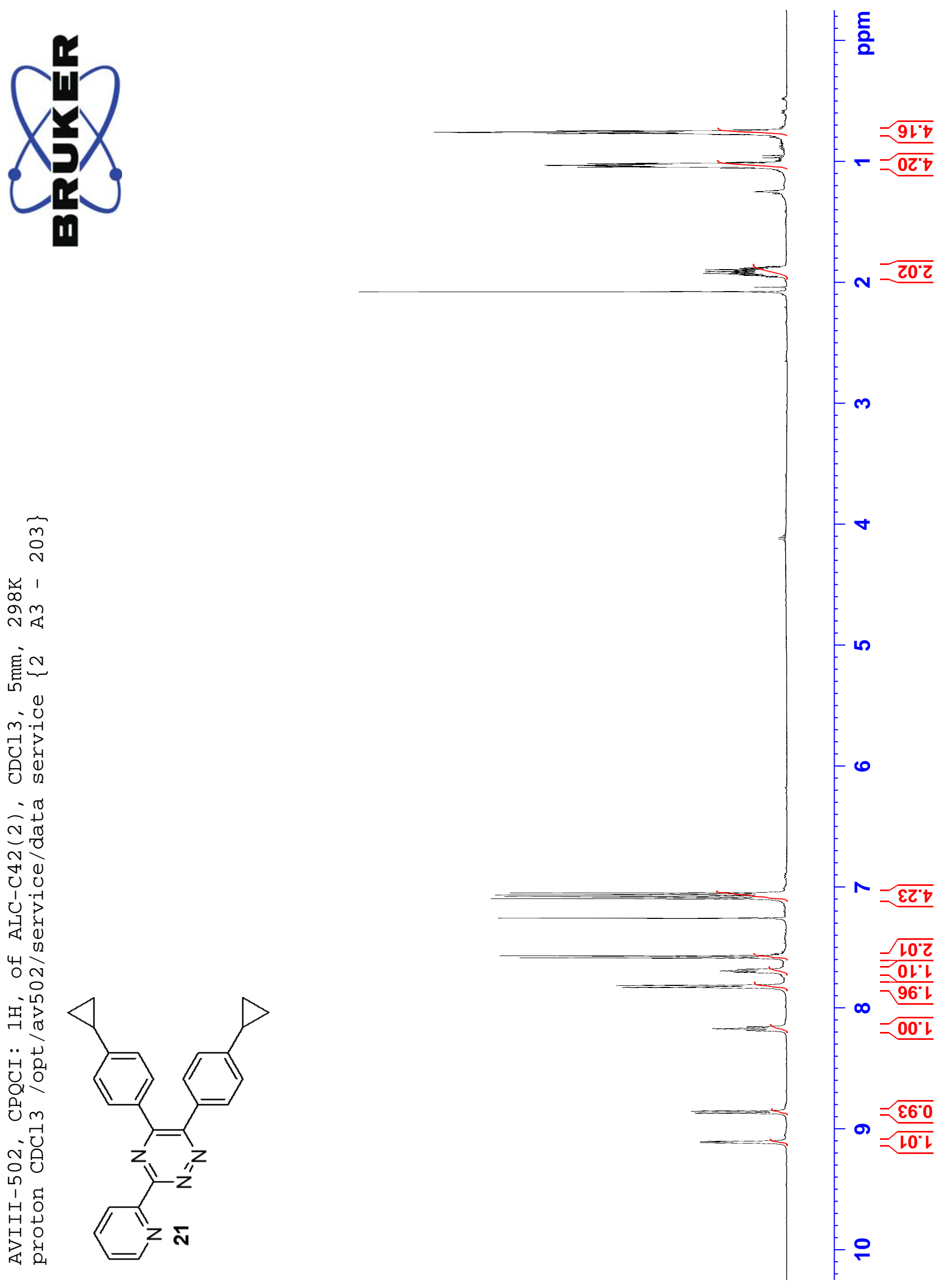

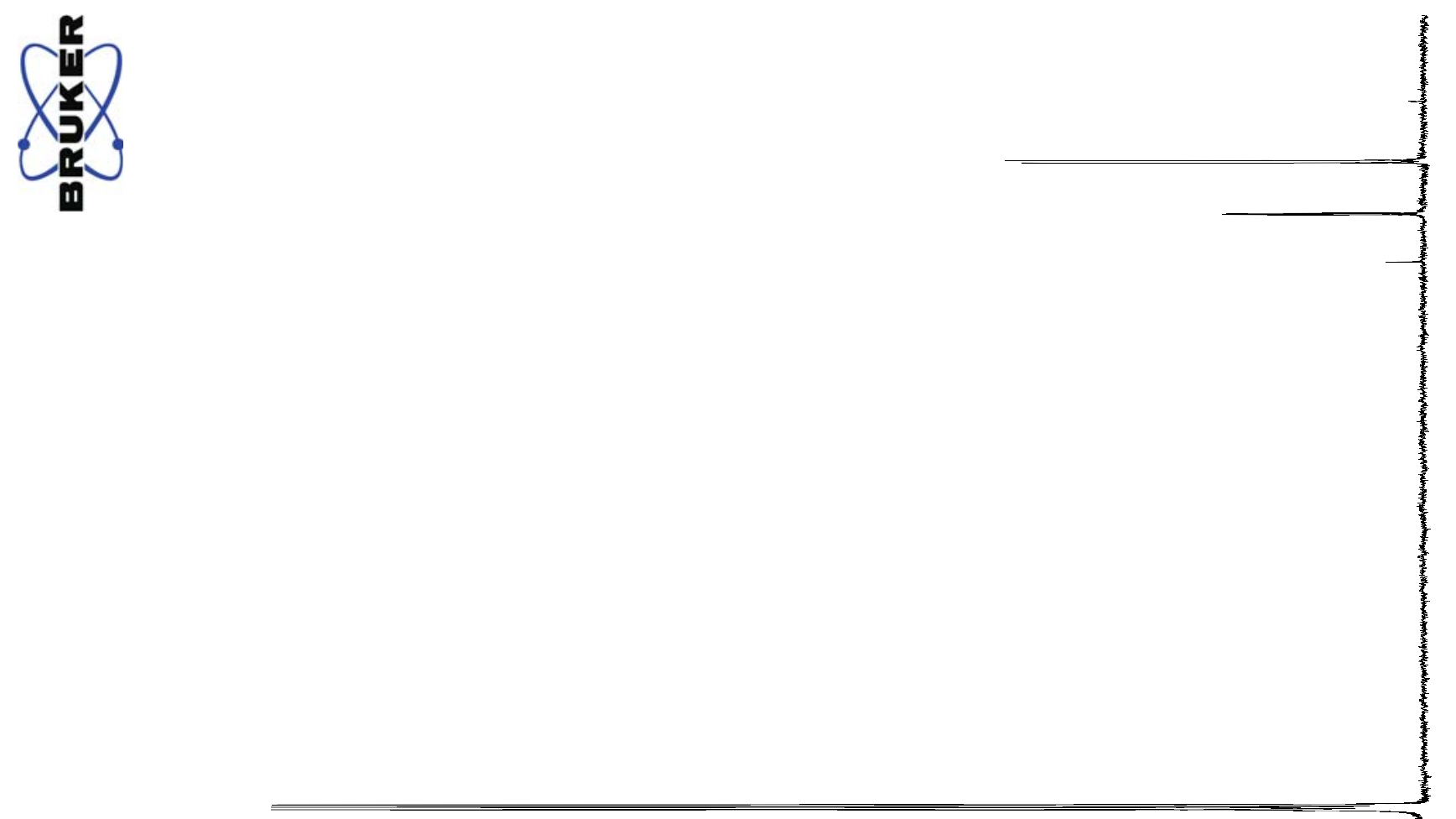

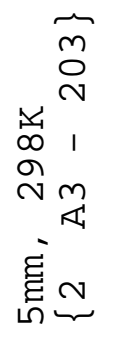
엄 กิธ 중

गे

ن

ن. \& $\frac{1}{0}$ 40

ते ن

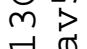

풍

벙응

잉

on ชั0

๑

'등

법을

$\gg \frac{1}{\sigma}$

GG'

२8.9टโ

$8 \varepsilon \cdot 6 z T$

○७ "

GI" ZEL-

$6 L \cdot 9 \nabla \tau-$

9t.87t

90.99I $\longrightarrow$

ZL. $\angle S I \longrightarrow$
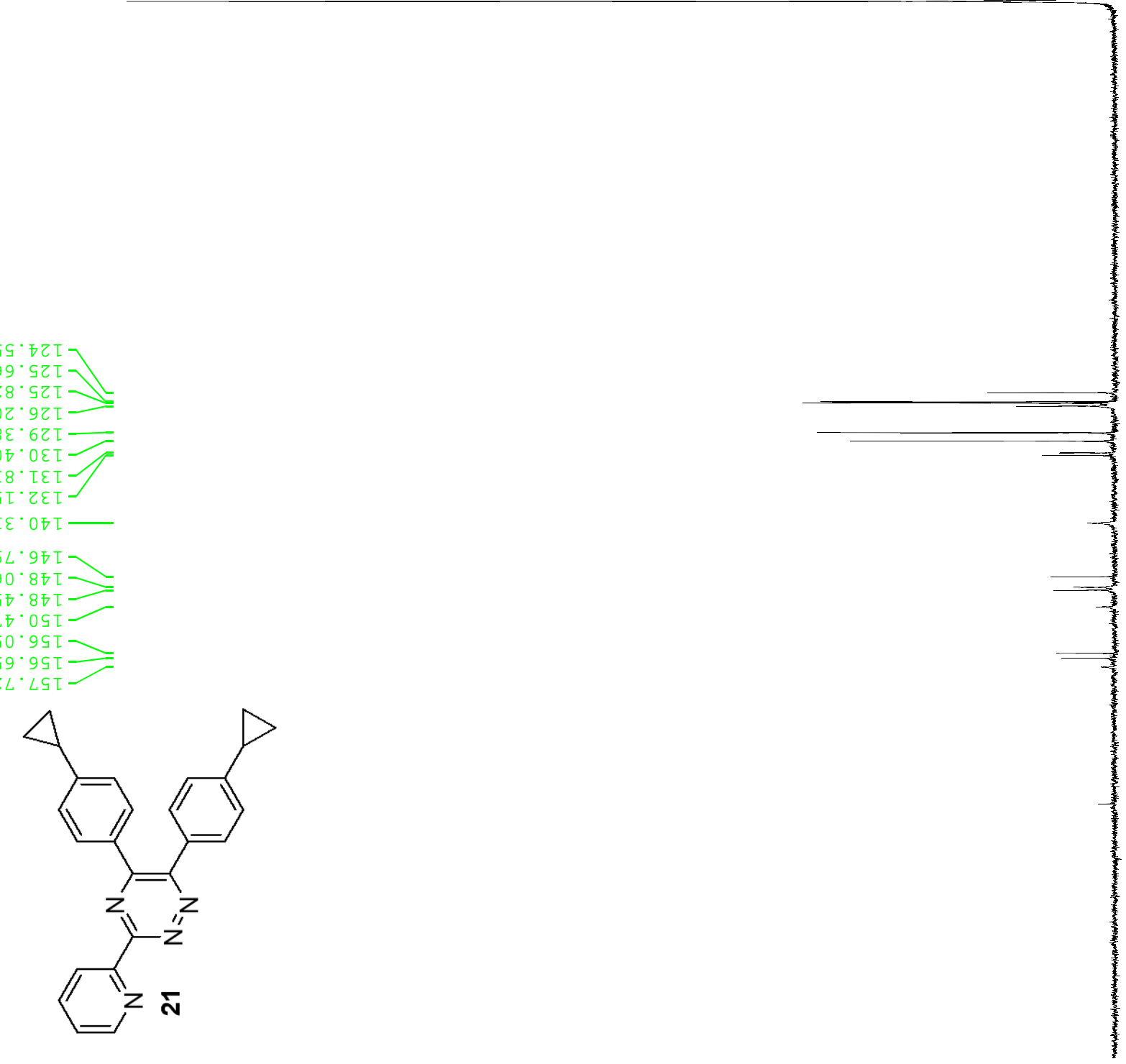

$-\frac{\varepsilon}{2}$

욱

- 으

ก

우

유

8

웅

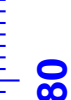



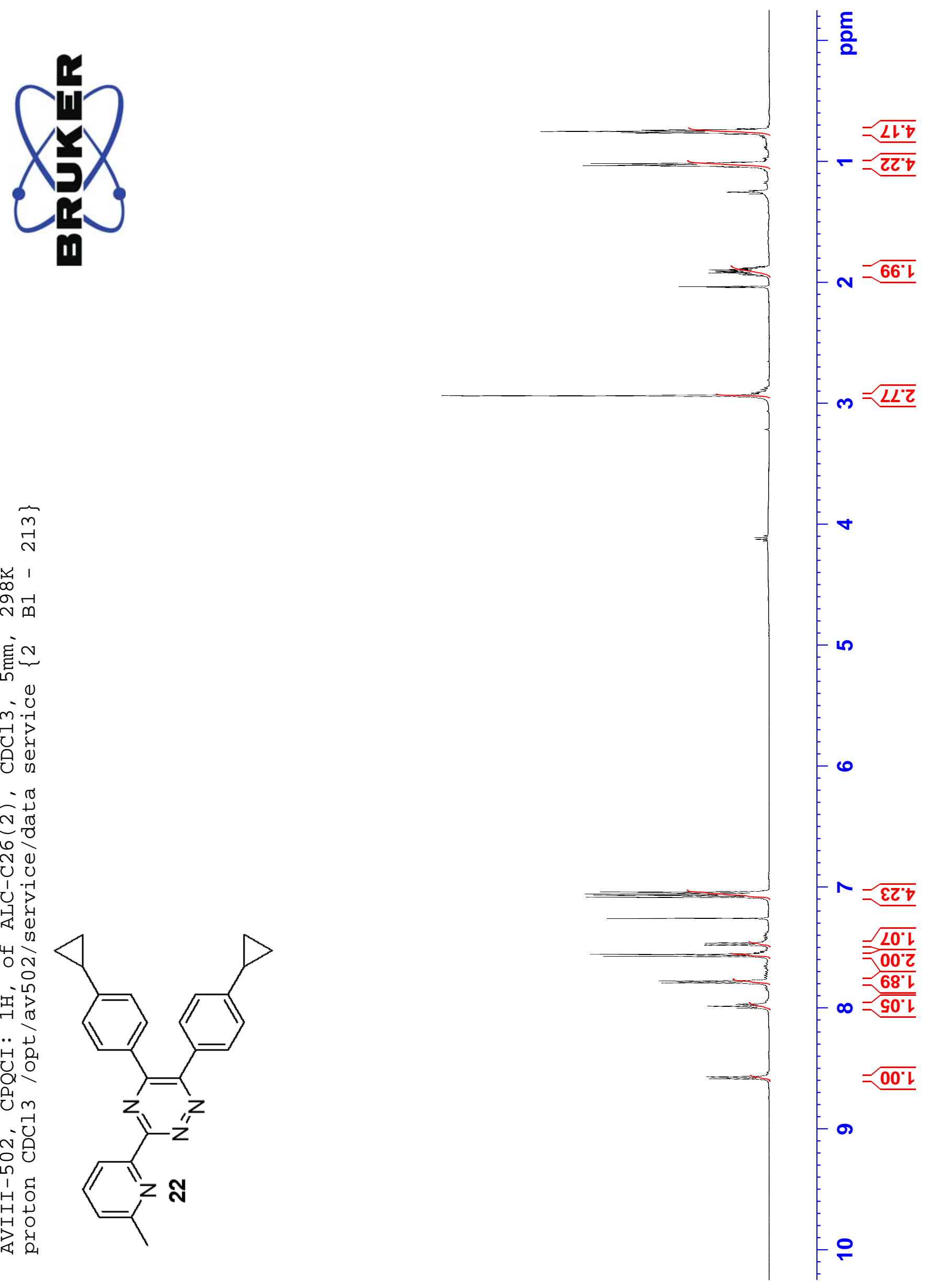

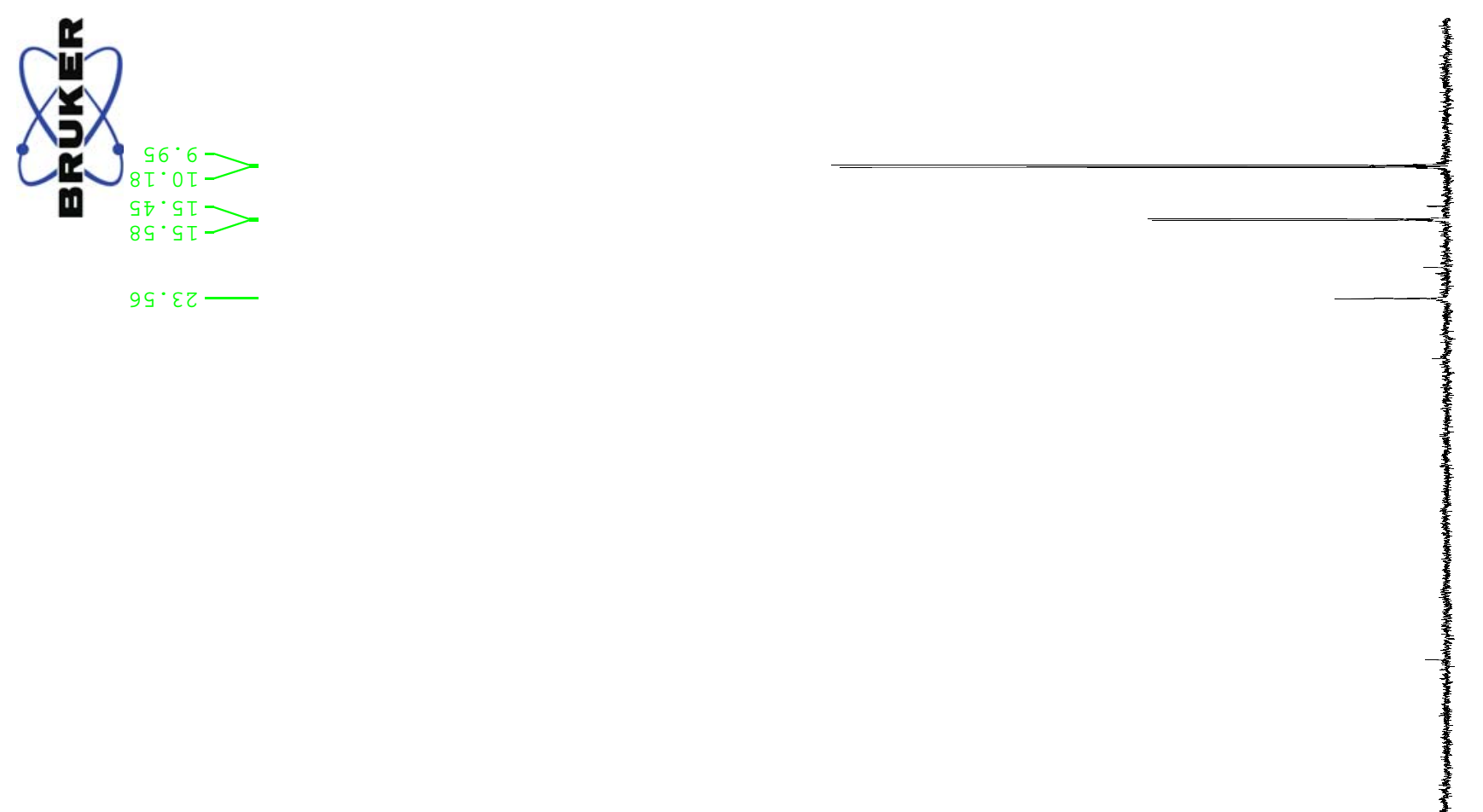

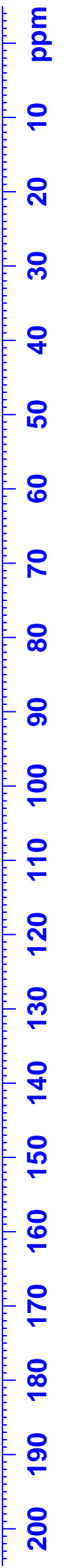
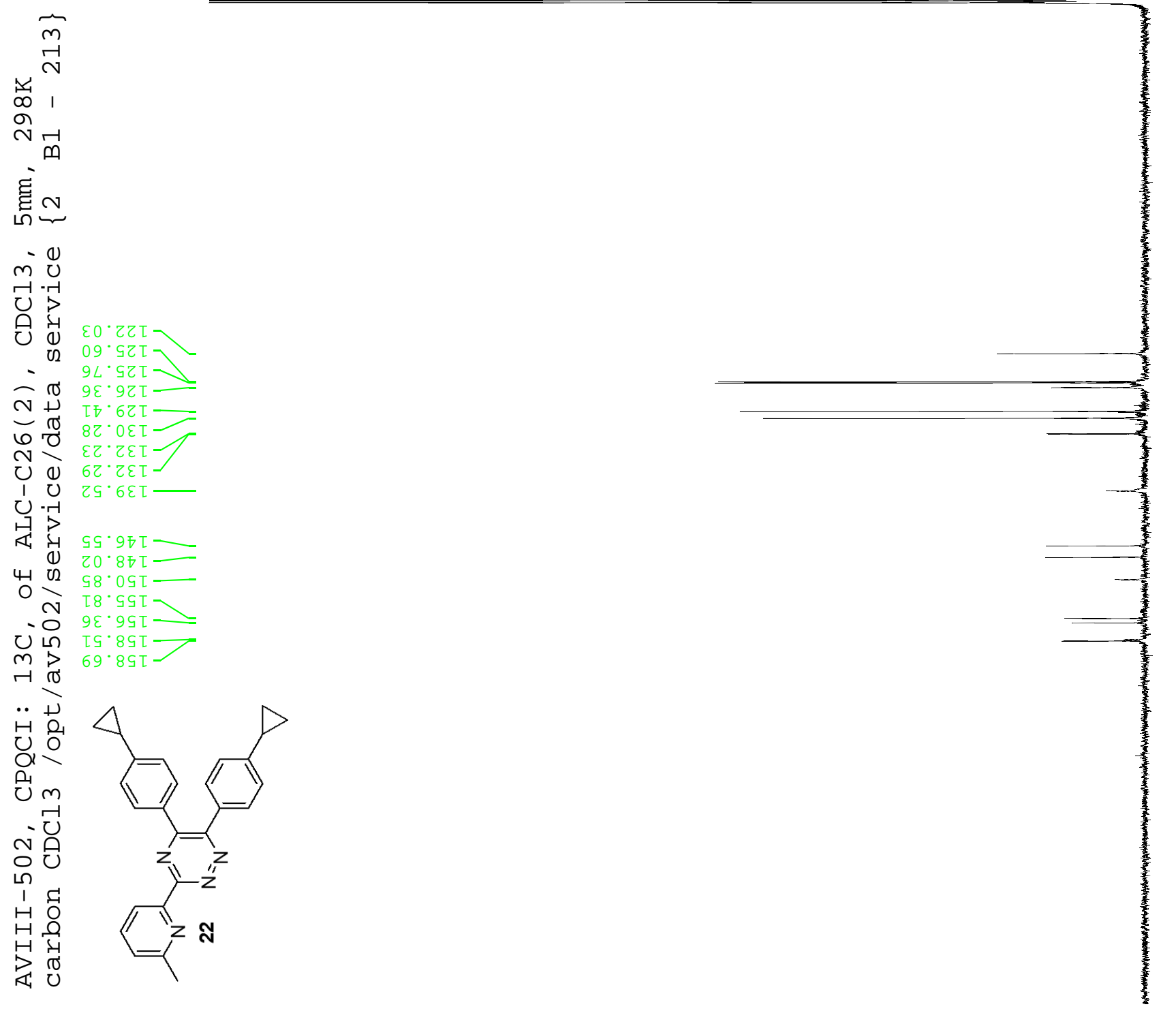

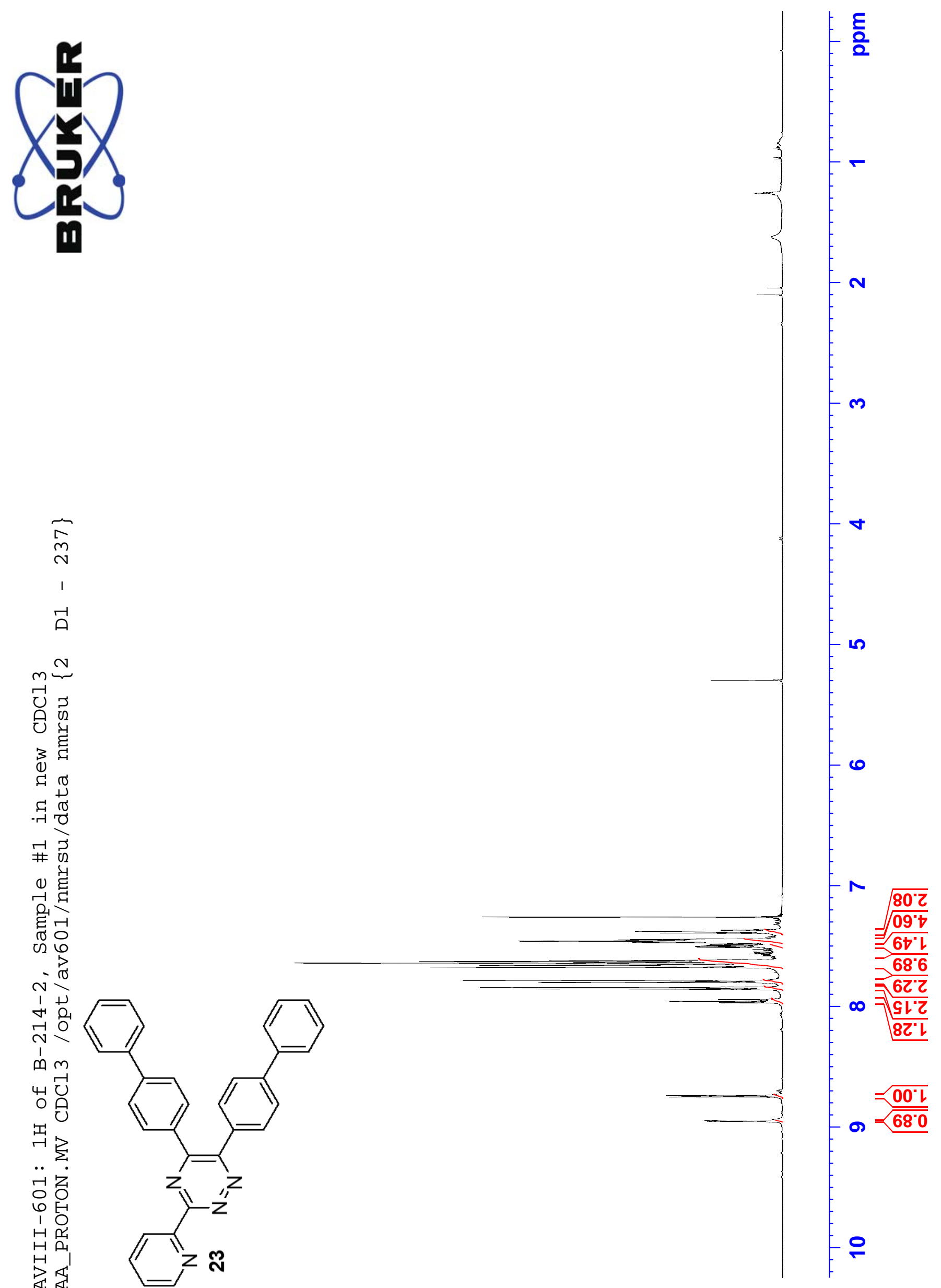

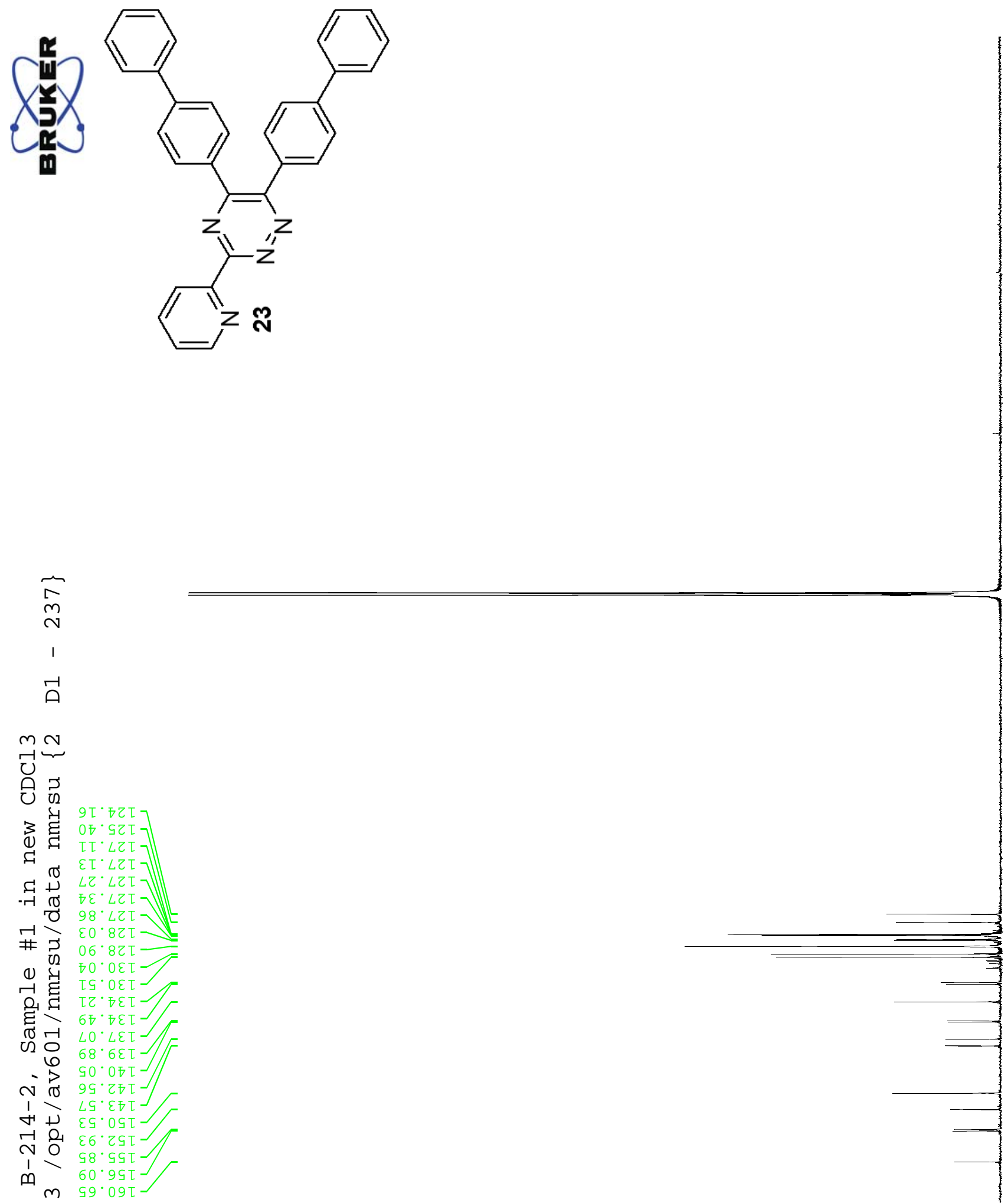

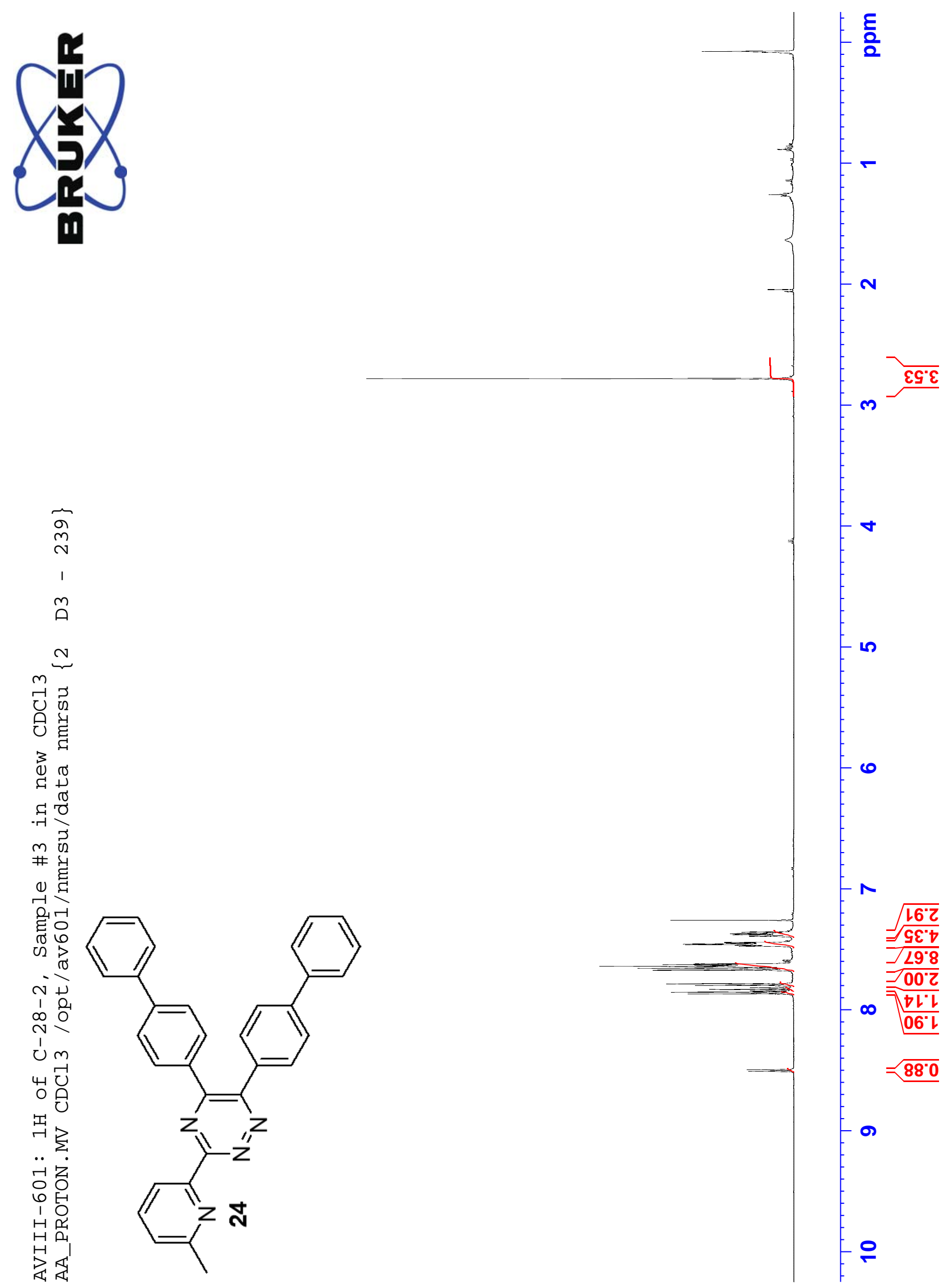
鄷

$88^{*}$ わ乙

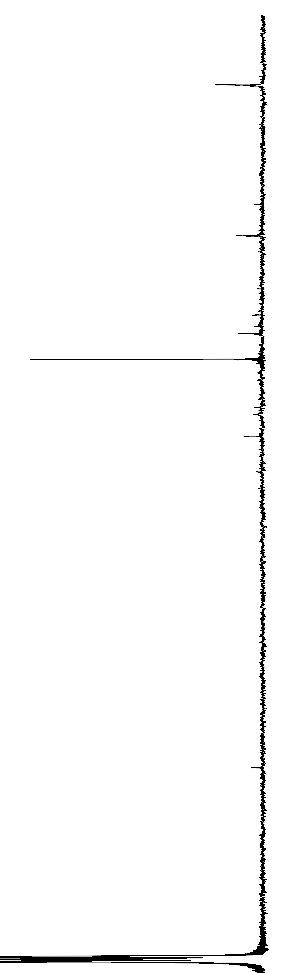

๙্ণ

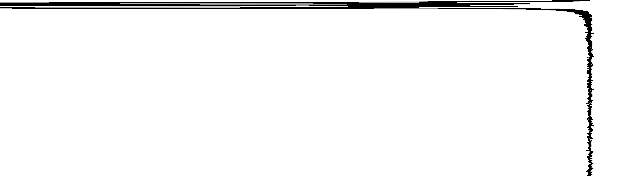

อุ
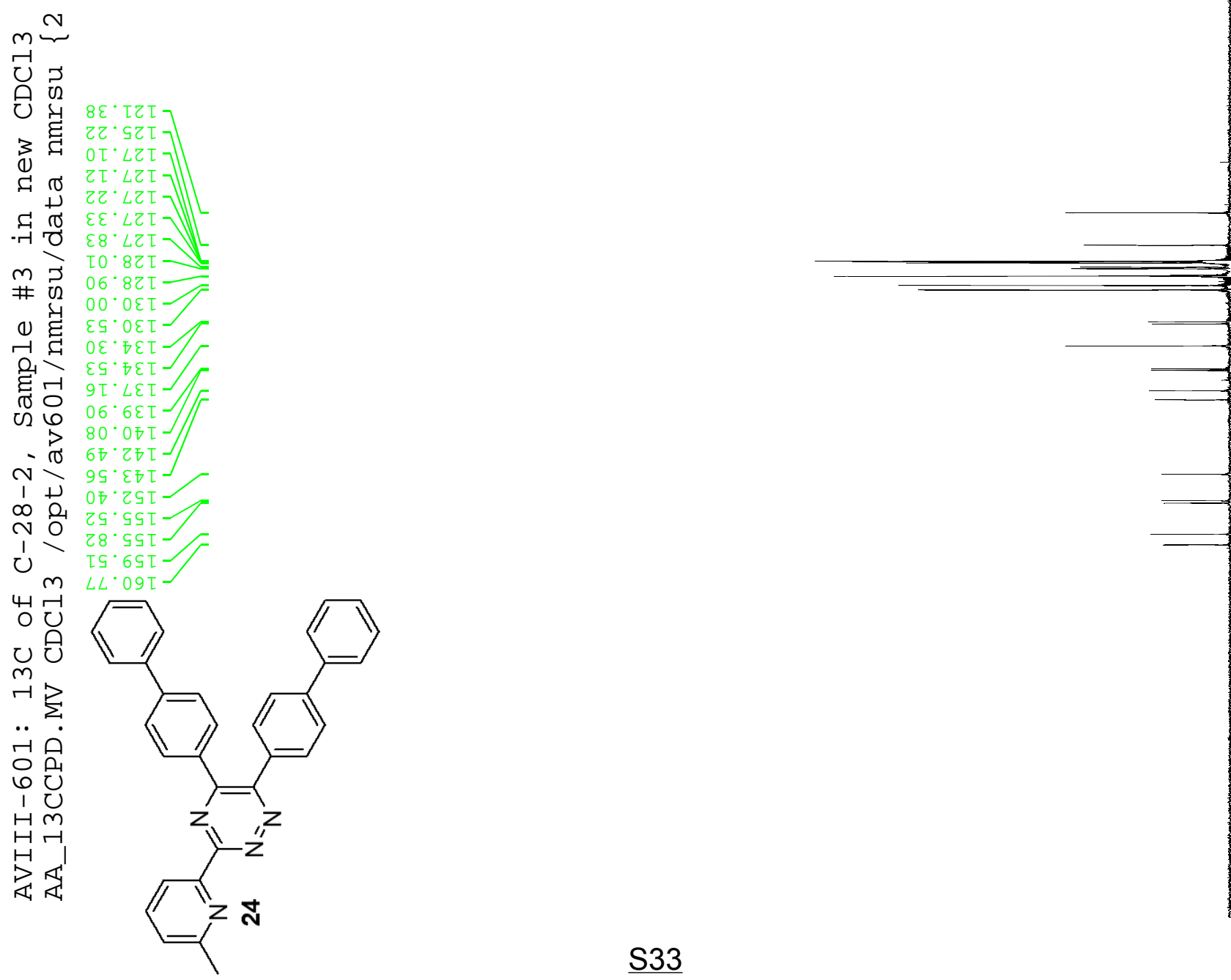

$\frac{\varepsilon}{2}$

음

유

웅

\&

유

8

$-$

$\infty$

웅

응

욱

옥

웅

웅

ㄱ

욱

웅

$-8$

울

$\infty$ 

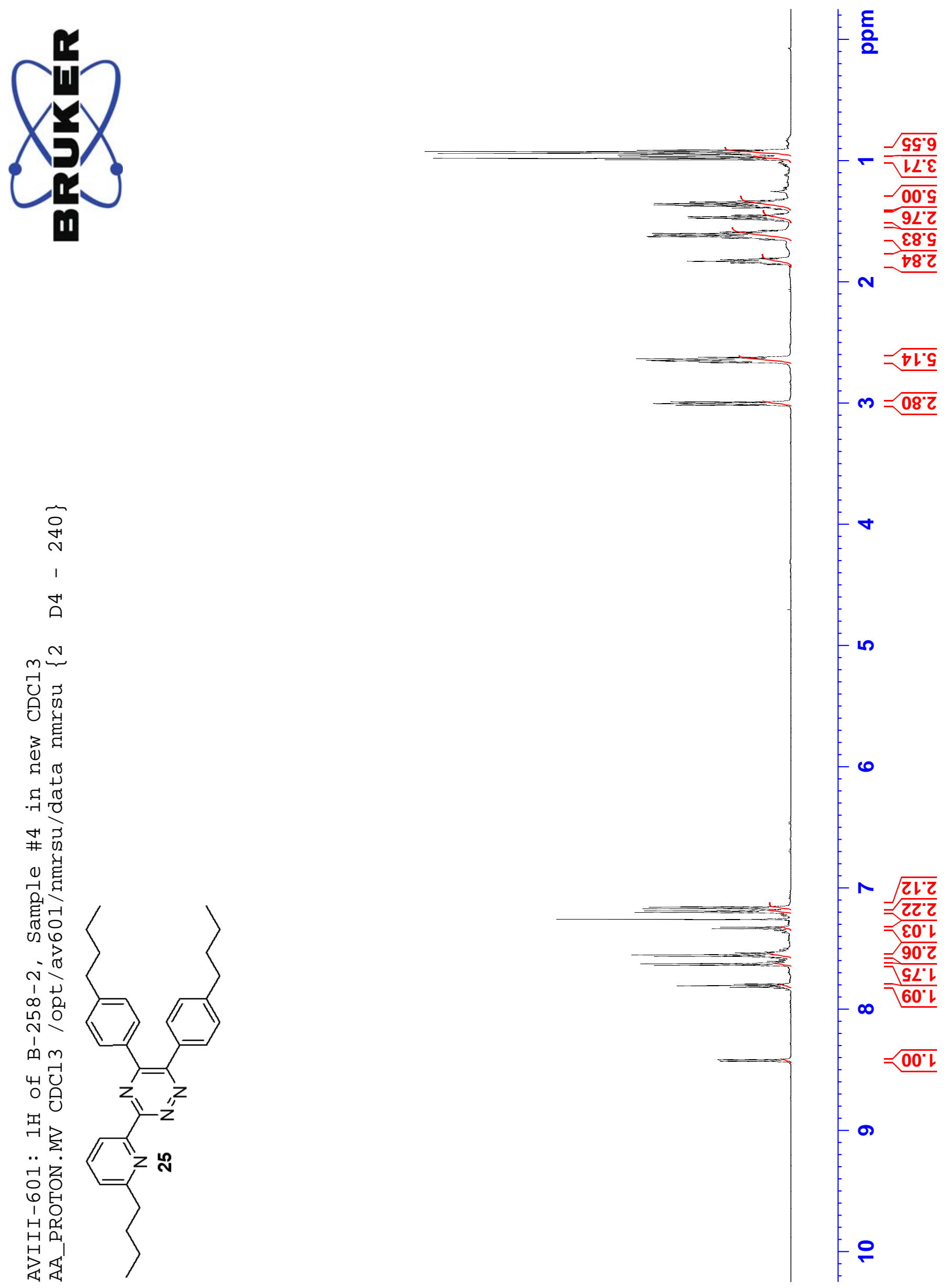

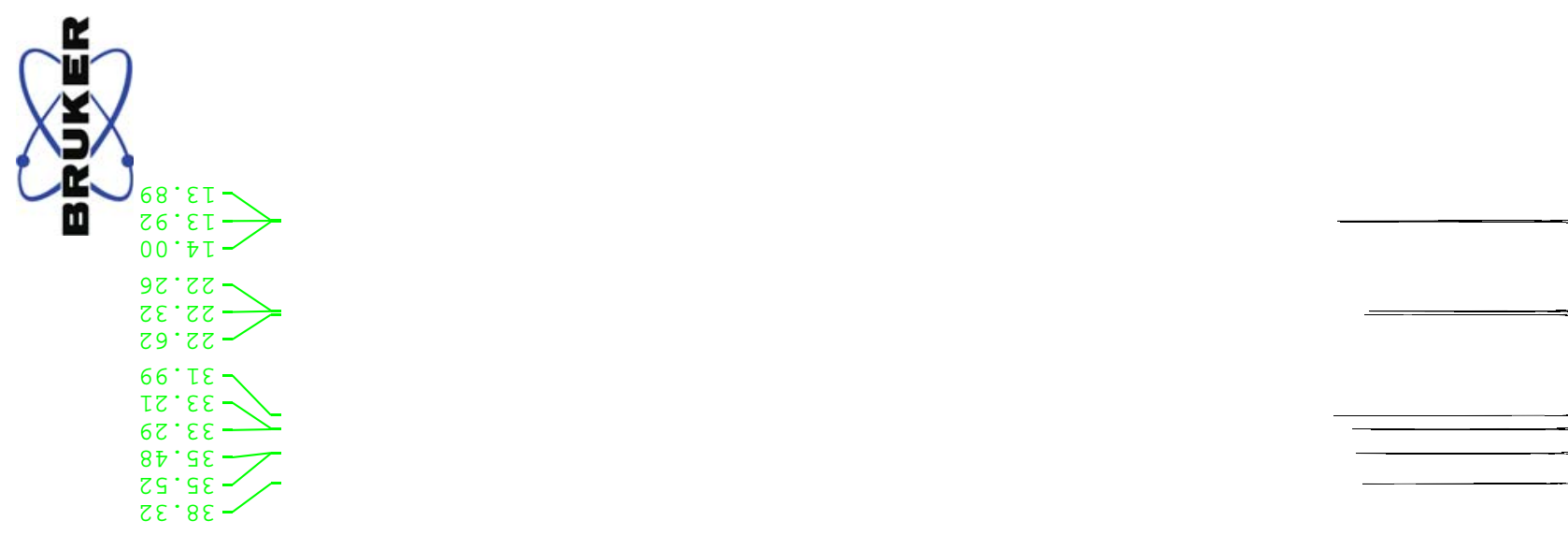

$\frac{\varepsilon}{2}$ 음

$\stackrel{\overbrace{}}{\stackrel{8}{+}}$

I

ষே

$m N$

거

仓

उ气 ह

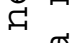

$\therefore \frac{\pi}{\frac{\pi}{0}}$

Z†'TZT

$6 T^{\prime}+D Z T$

\#ذ

$99^{\circ} 8 \mathrm{ZT}$

() ᄂ 26.6zI

기 Z6.2हt

ह्ञ $86^{\circ} 9 \varepsilon \tau-$

ஸळ $\varepsilon L " \nabla t=$

Ni⿱

'ो

I9'Z9T-

$\infty+$

\&L'GST

응

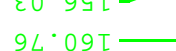

$\infty$

$9+\cdot{ }^{\circ} \cdot$

4

บบ

m

$\because 2$

ㄱํㅇํํ

10

Hल

간

¿
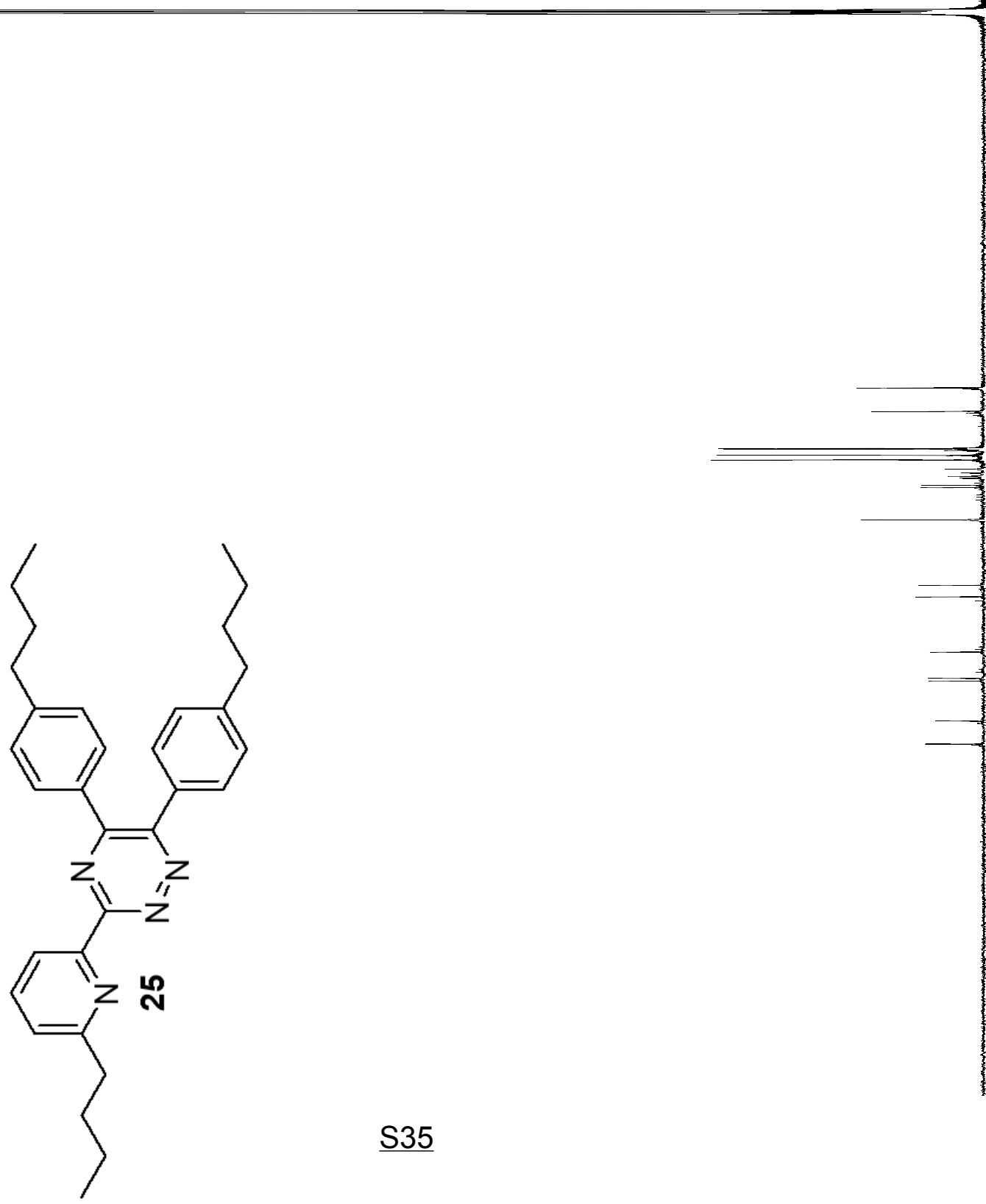

$=$

$\infty$ 


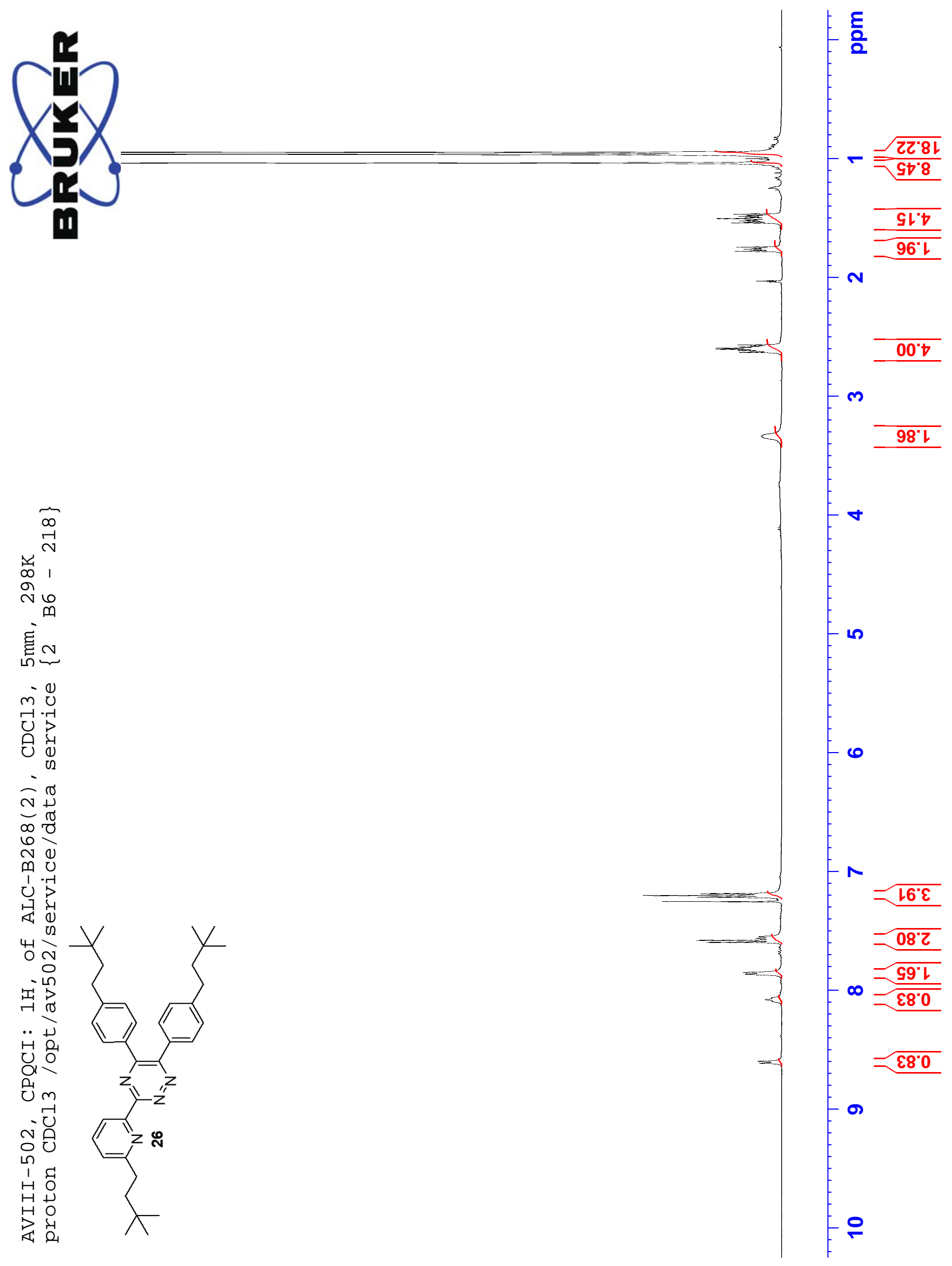


㩆
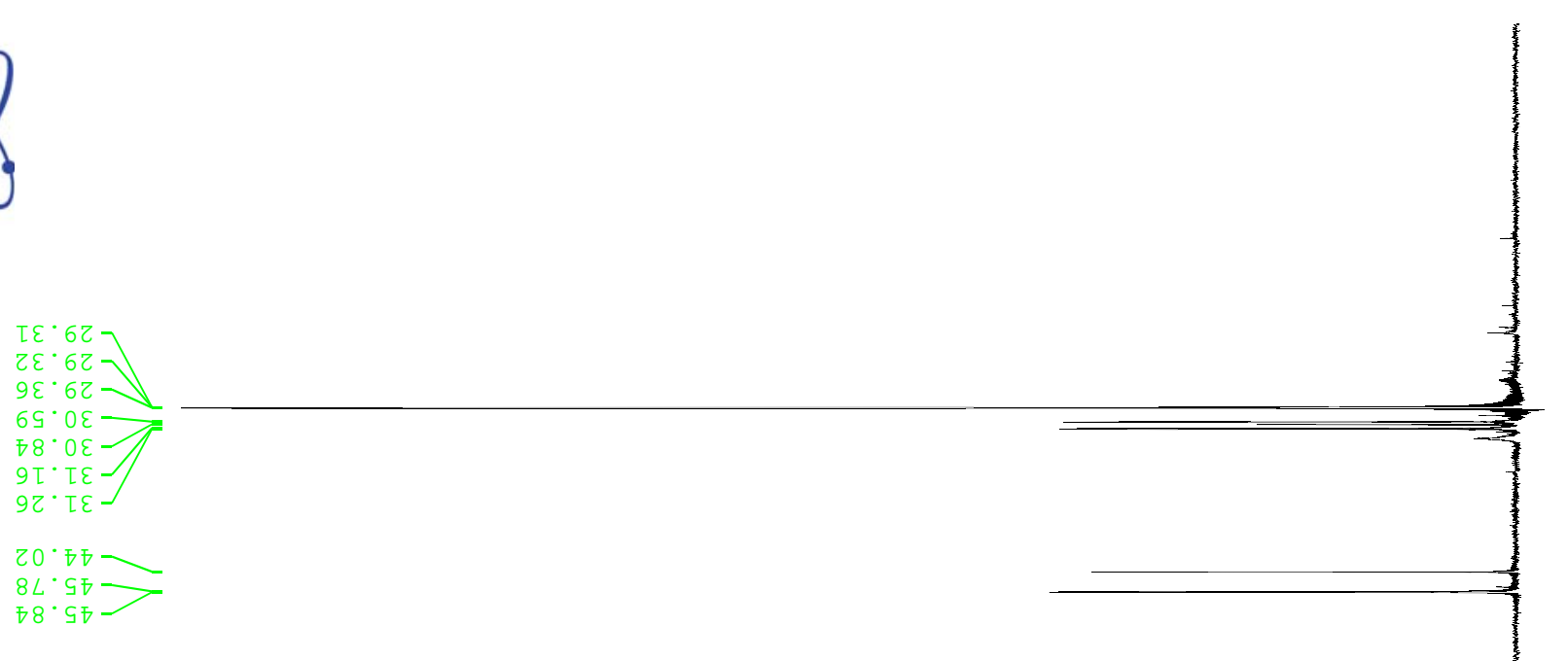

$\rightarrow 8^{\circ} \mathrm{G} \nabla$

틍

๙্

I

$r^{\infty}$

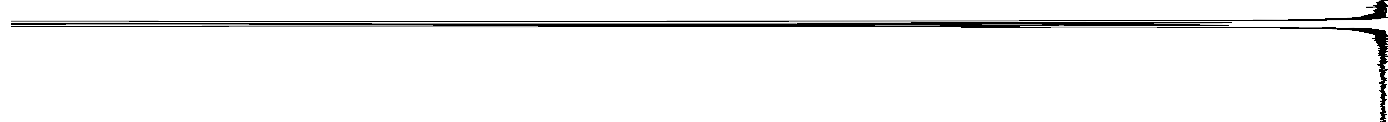

$\frac{1}{\infty}$

N

हो

लंख

บั

บ늉

กิ

ㄴ.

$\infty>$ Ig.6ZI

0 ᄂ L

(1) Gt. ZEL

๓

0 न

$\varangle$

06.9t5 -

पे

능

.

닝

90.99T

ع9.9ST

...

$\because 0$

엉

U

겅ㅇํㅇ

पㅇ

벙

반

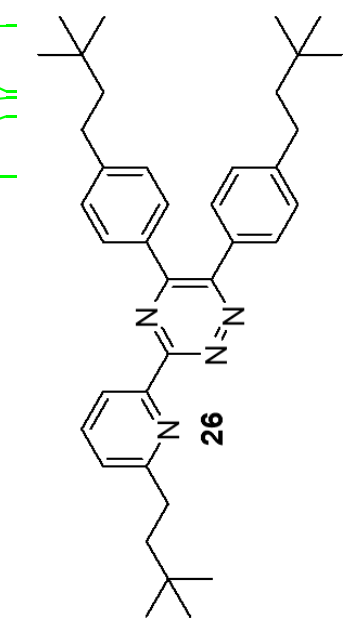

$\angle t^{\circ} \varepsilon 9 \tau$
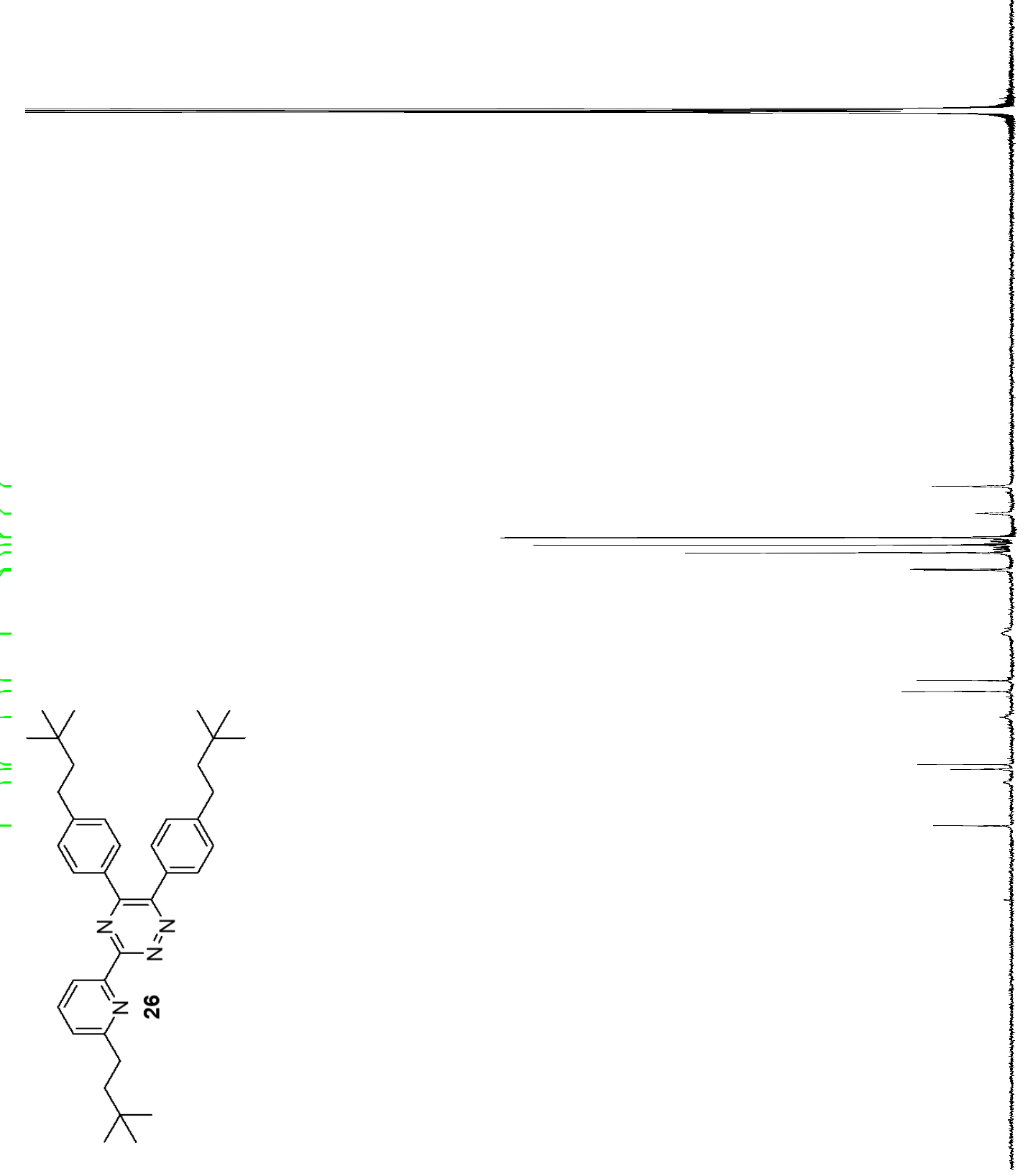

8

-

$\infty$

요

웅

음

옥

E 욱

웜

음

욱

욱

욤

욕

웅 


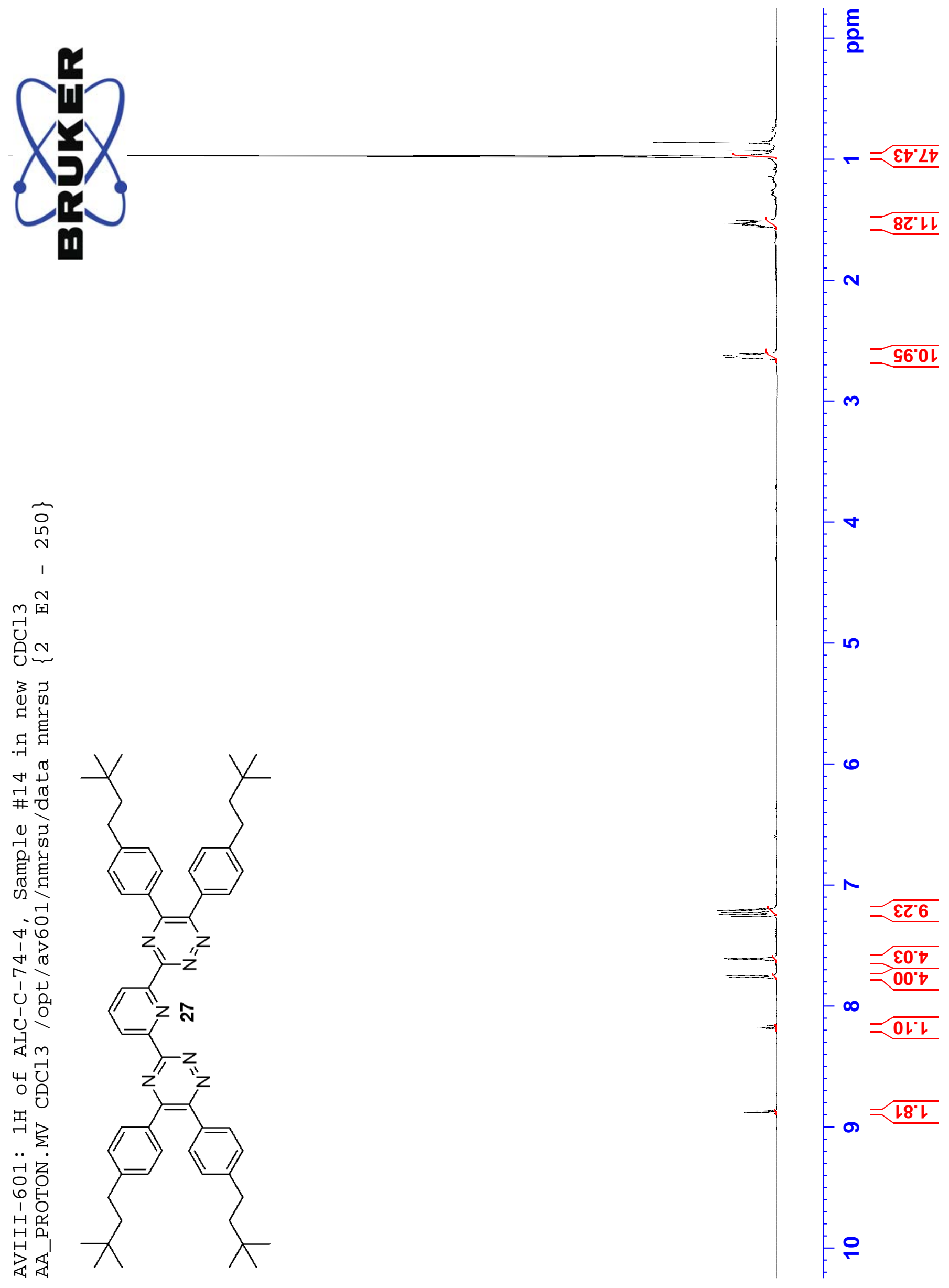




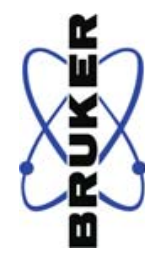

$\nabla \varepsilon \cdot 6 \tau$

$\odot 9$ ०ध

$6 \tau$ 'LE

G8. $68^{\circ}$ St $\longrightarrow$

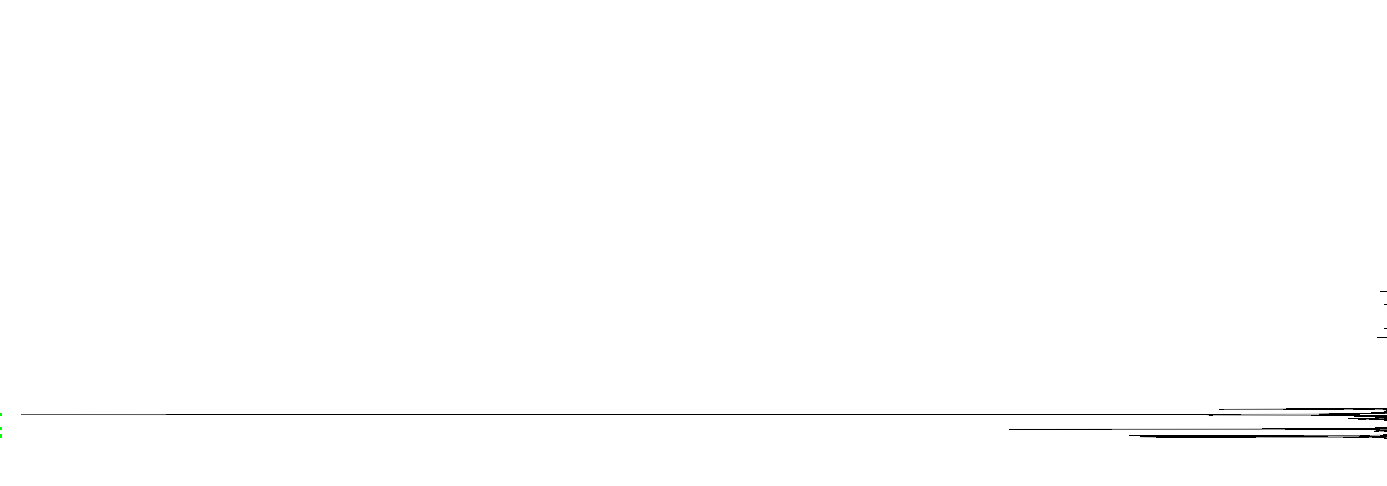

告
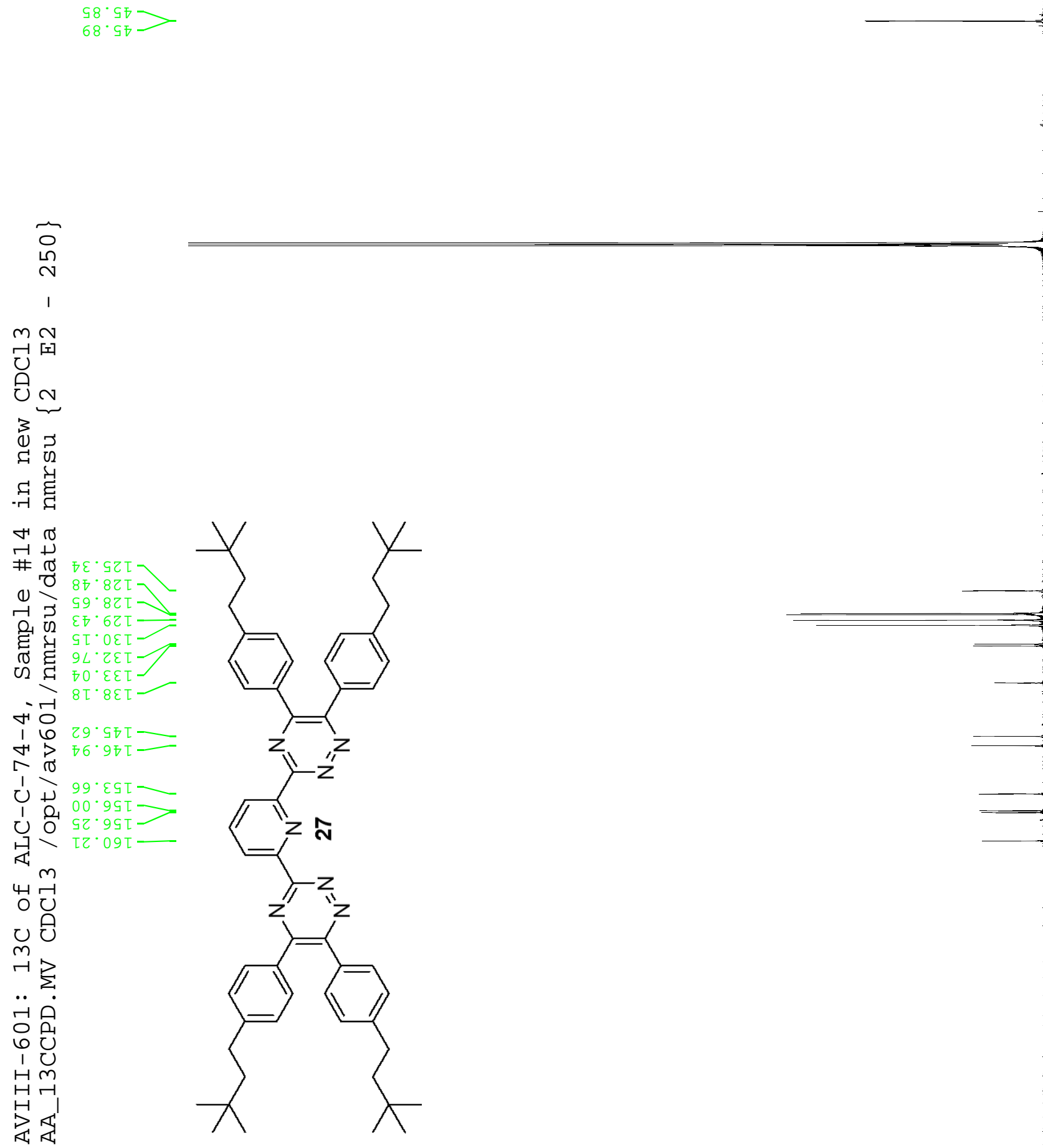


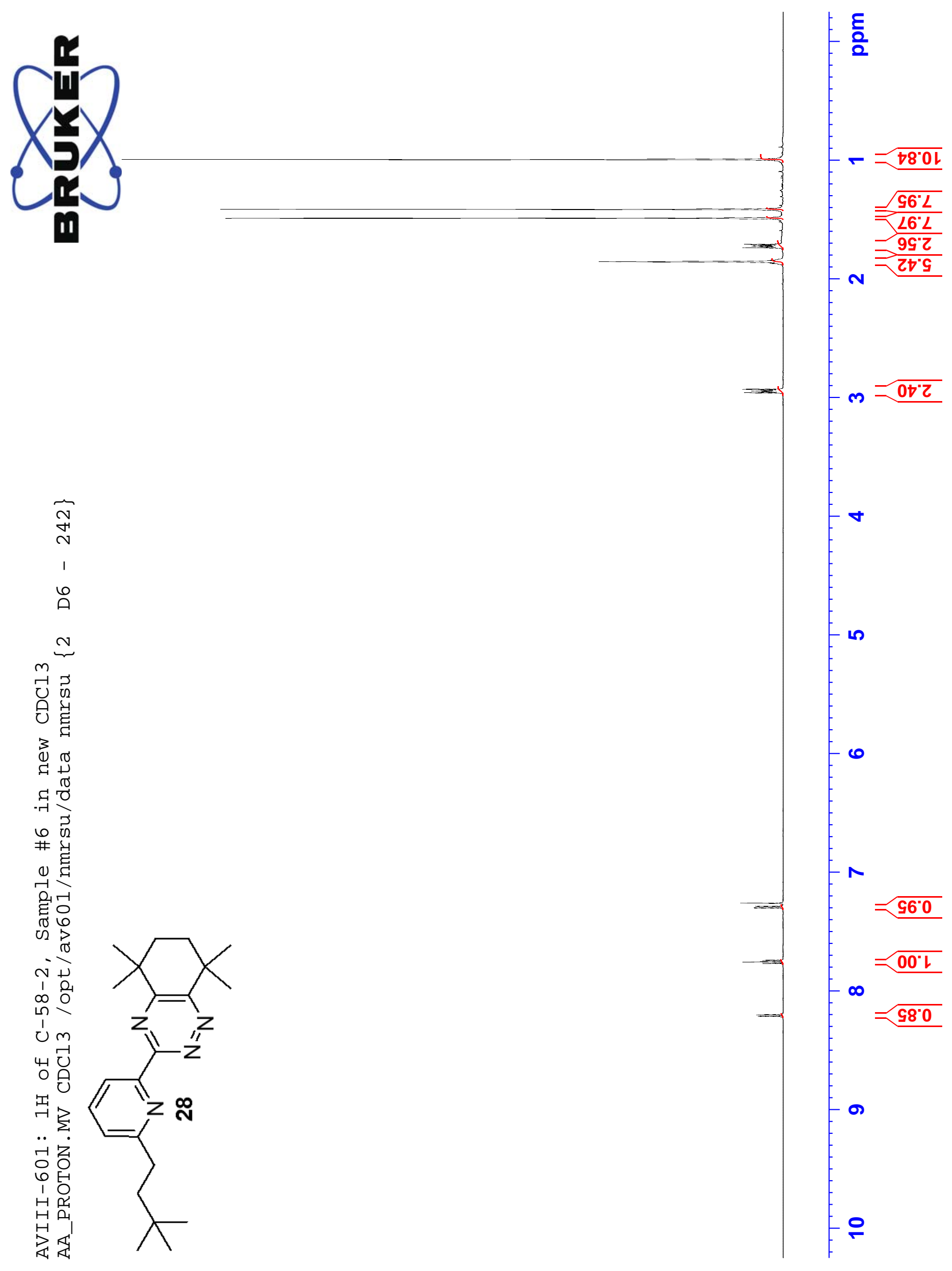


影

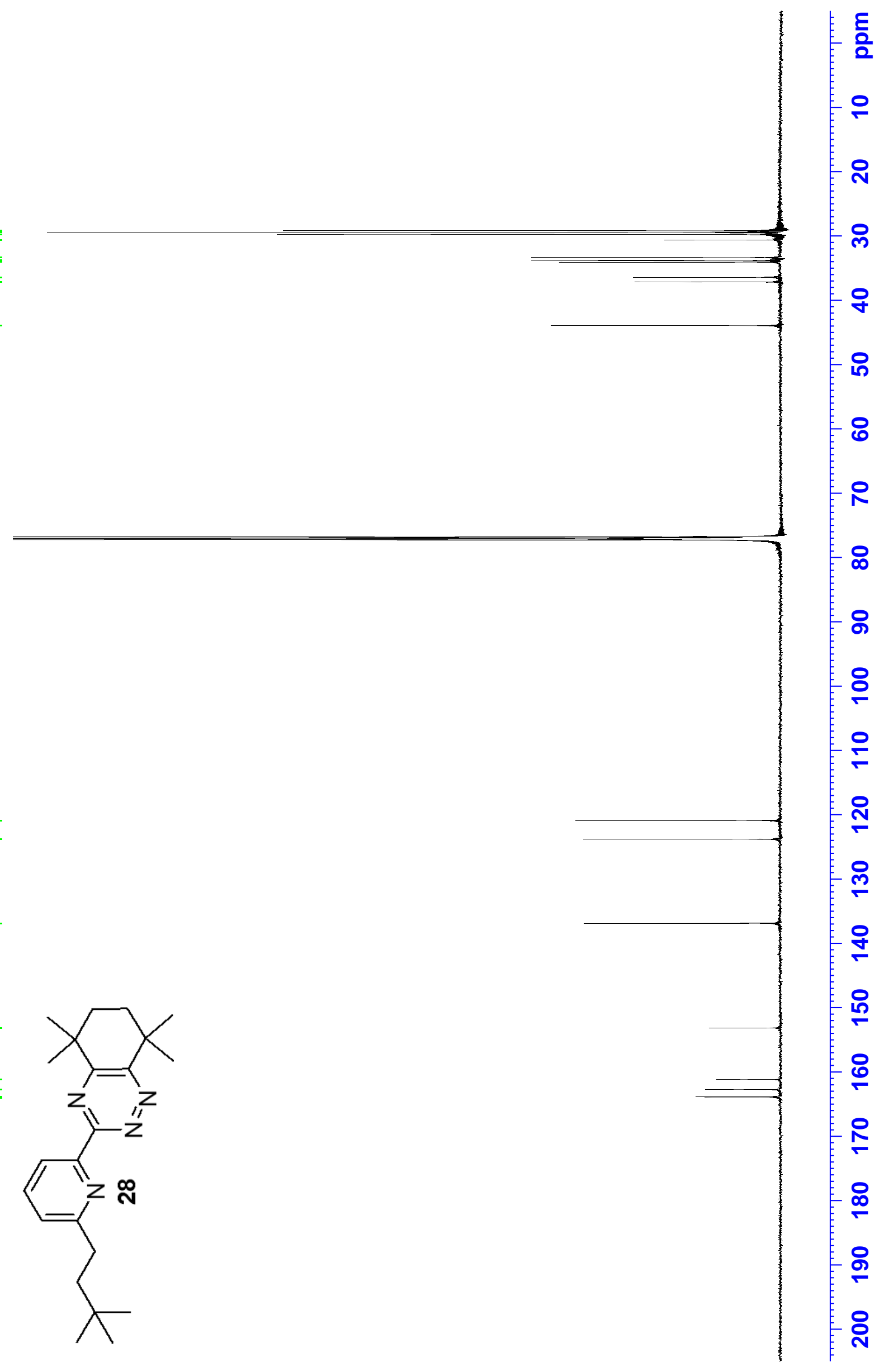



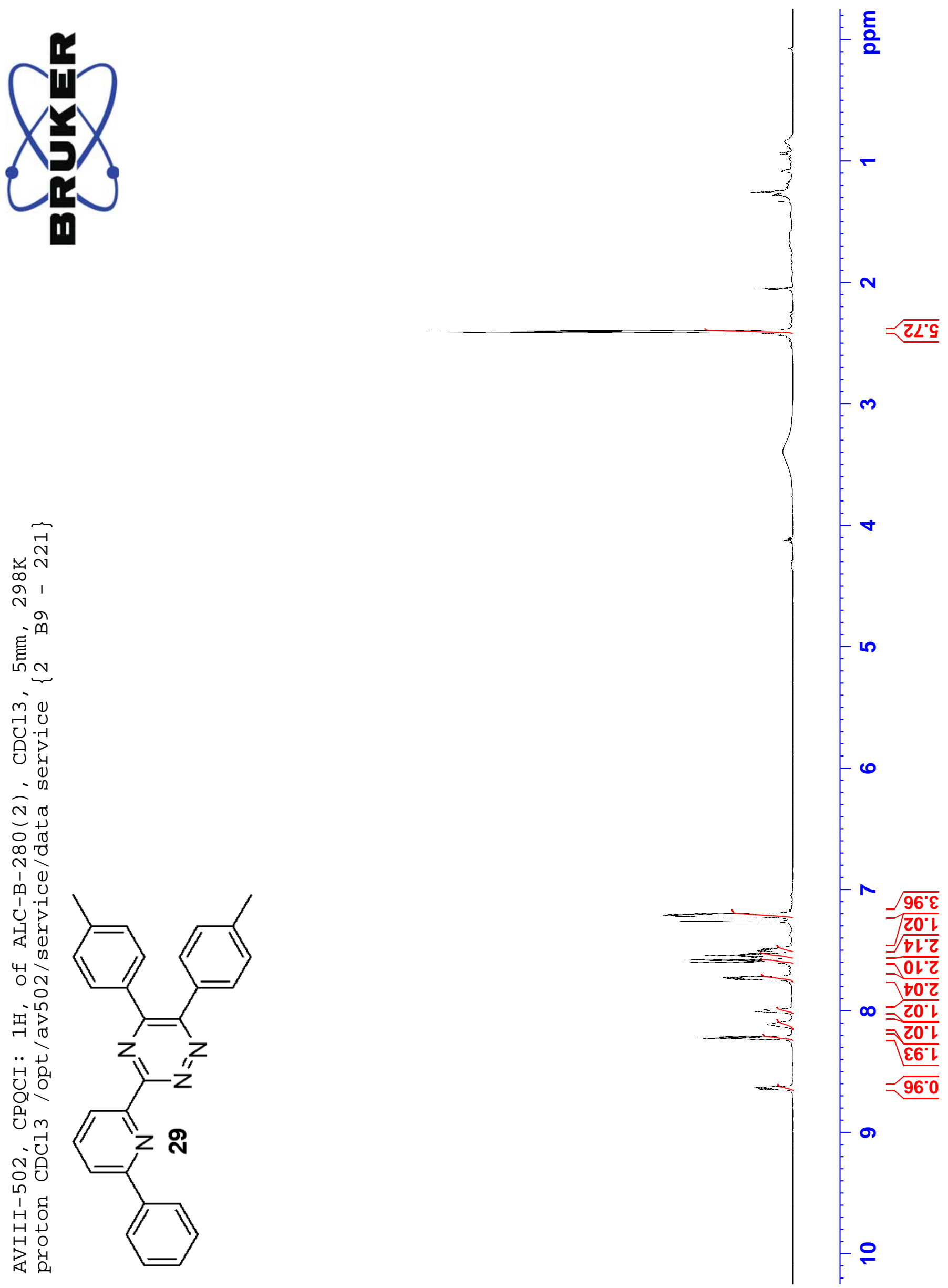

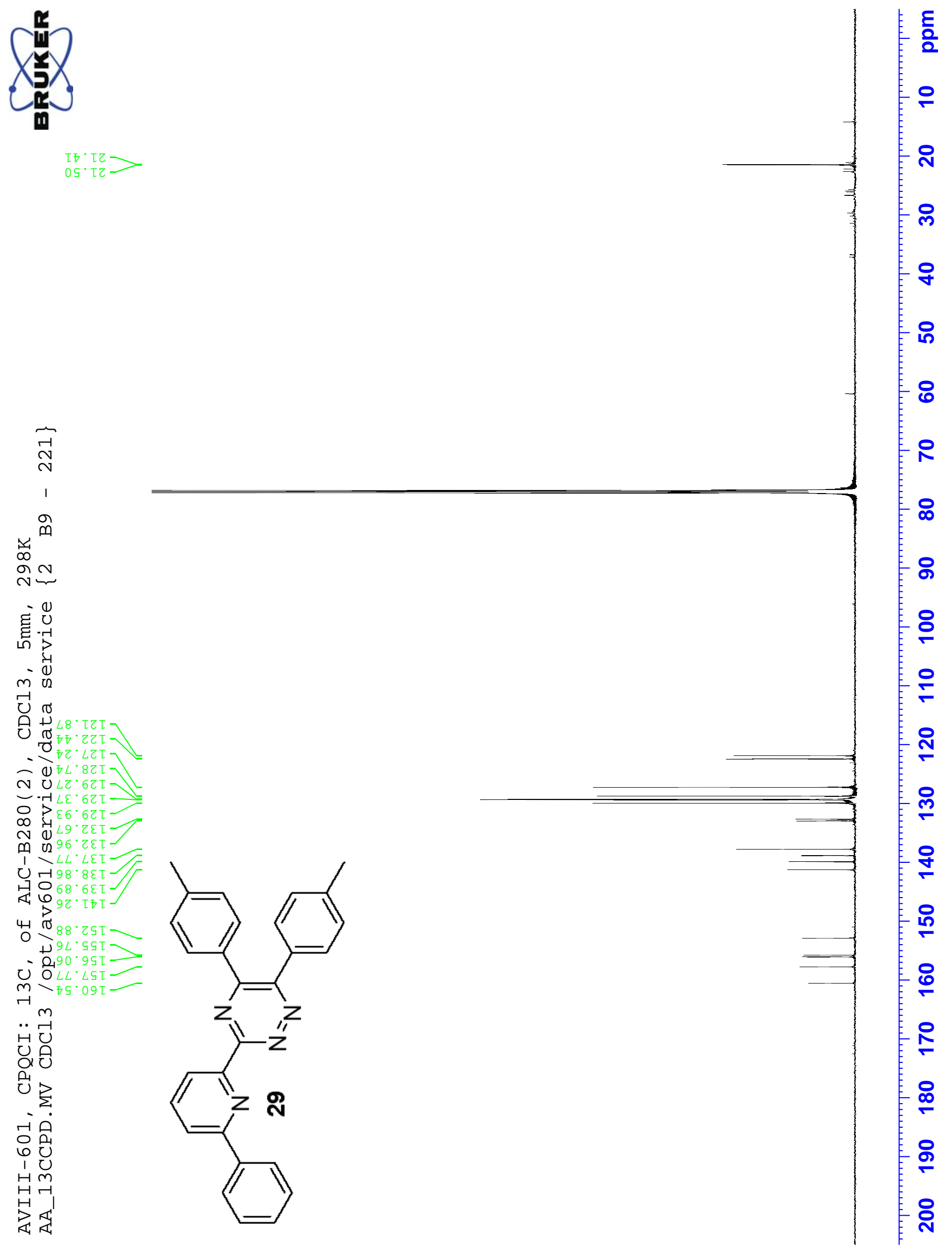


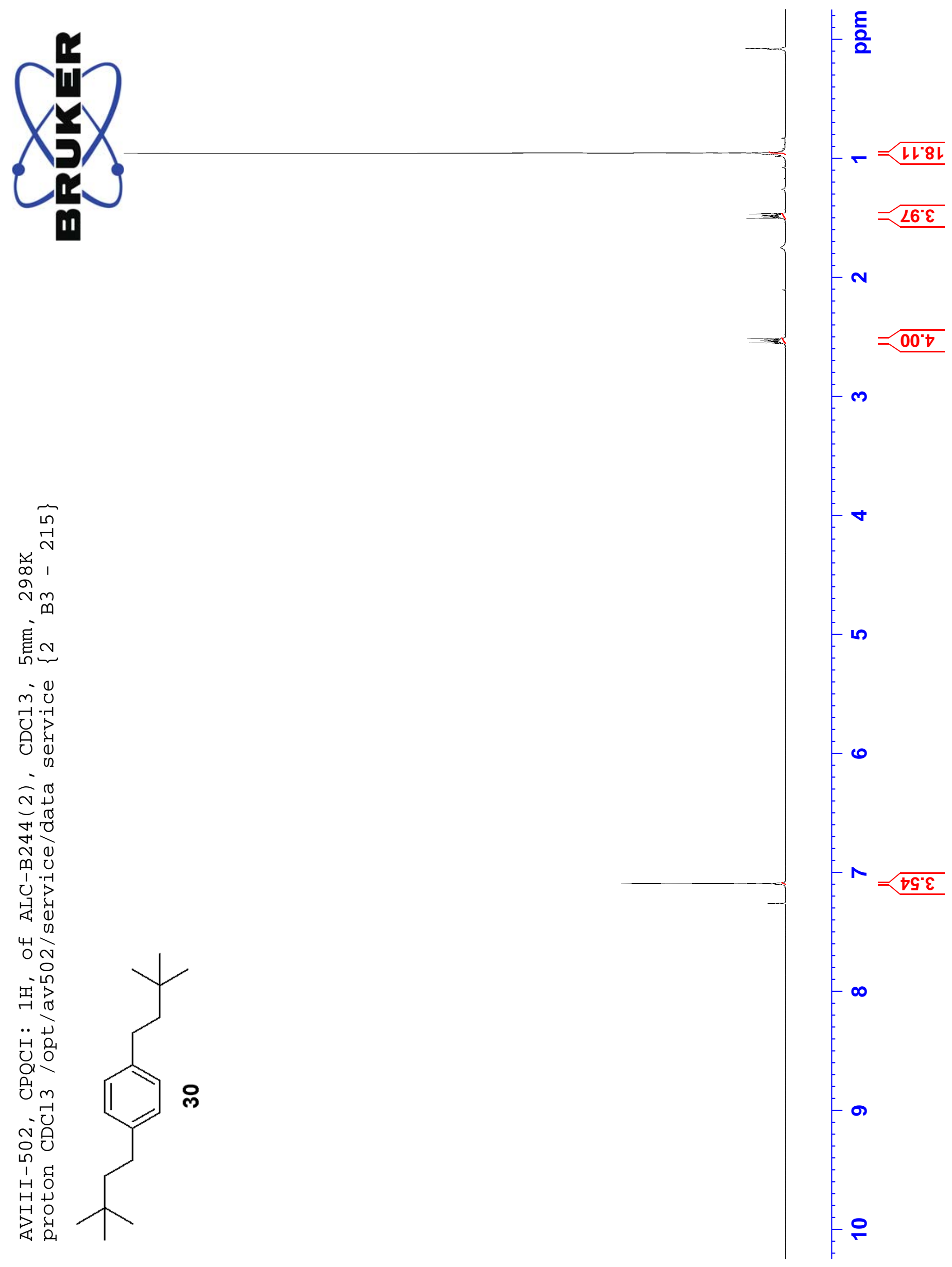


鄷

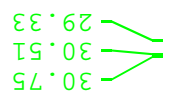

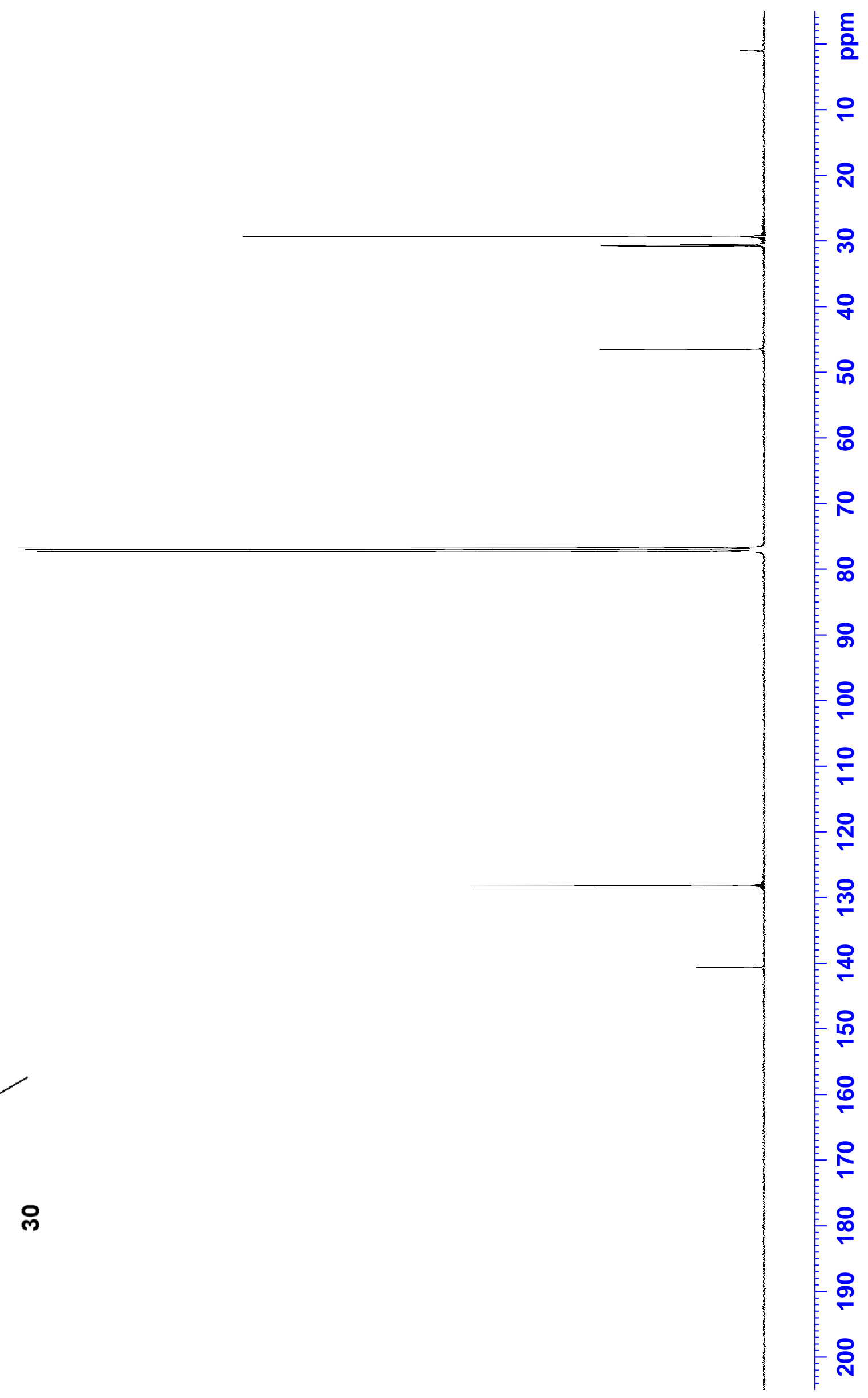

ํํำ

N

हึ

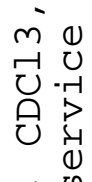

กิ

夹

(1)

نं. $79^{\circ}$ ำ —

$\frac{1}{4} \frac{1}{4}$

4 ज

نิ

บ

तળ

..

번응

这

त्र

จิ

问

벙

배은

\&

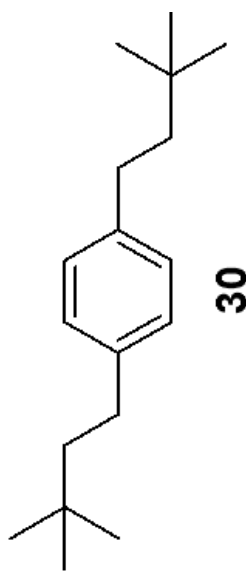



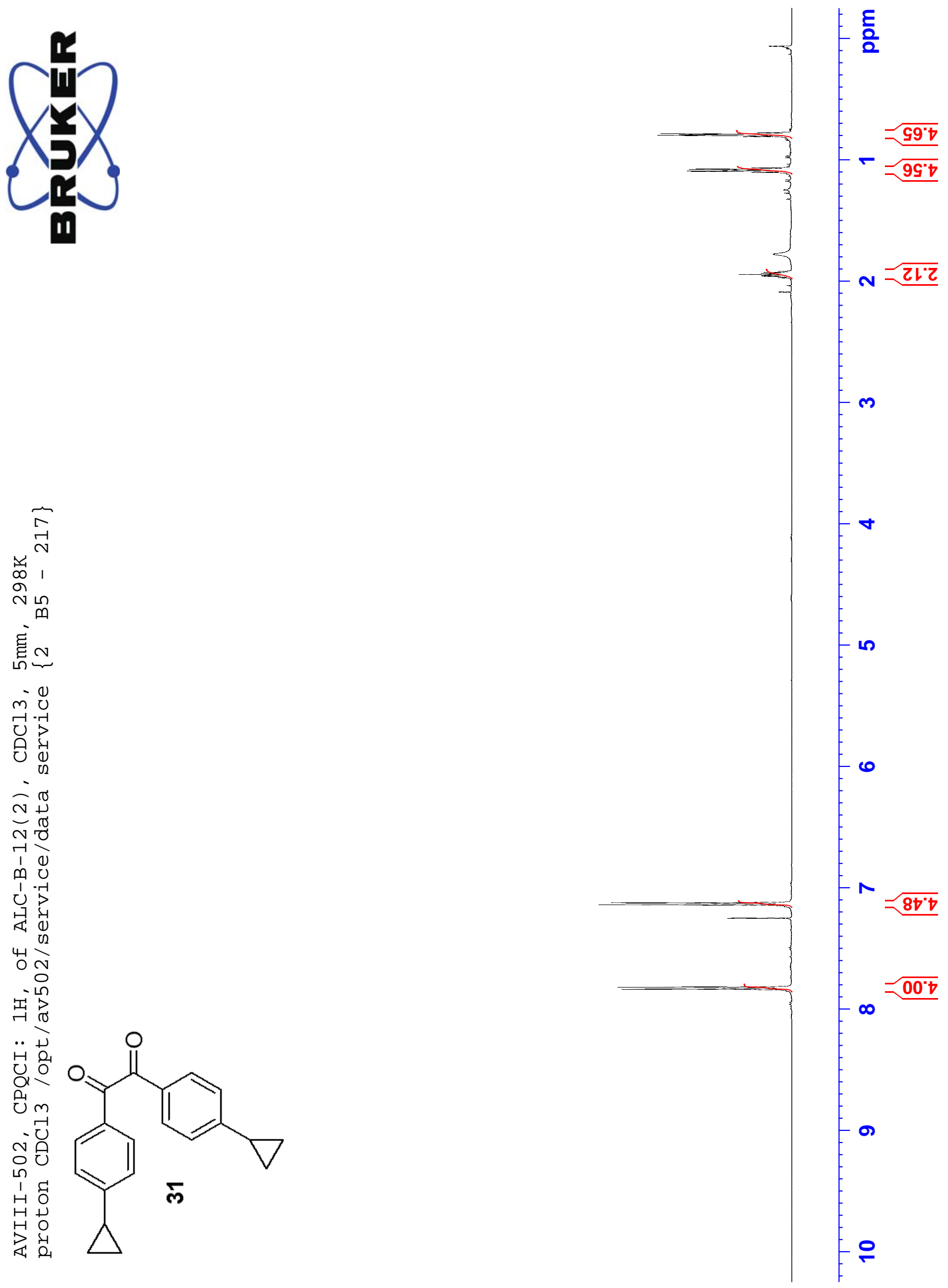
(11)

$\mid$ E 틍

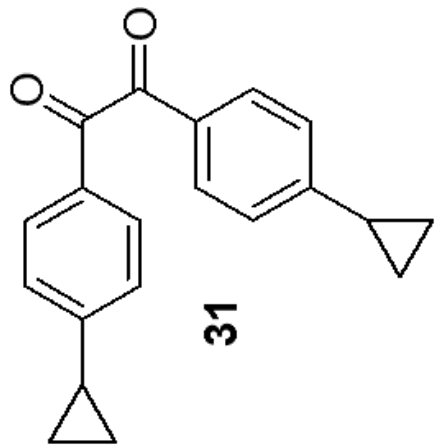

요

우

요

8

R

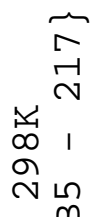

हً⿳亠二口犬

نั

กิ퓨

ก 응 8 -

तै

i.

\&

4 in

○ $89^{\circ}$ ZST

ن

$\underset{\sim}{m}$

..

$\because \because$

잉

Oे

-

N่

ำ

1 단

법응 $9 \varepsilon^{\circ}+65$

느

《证

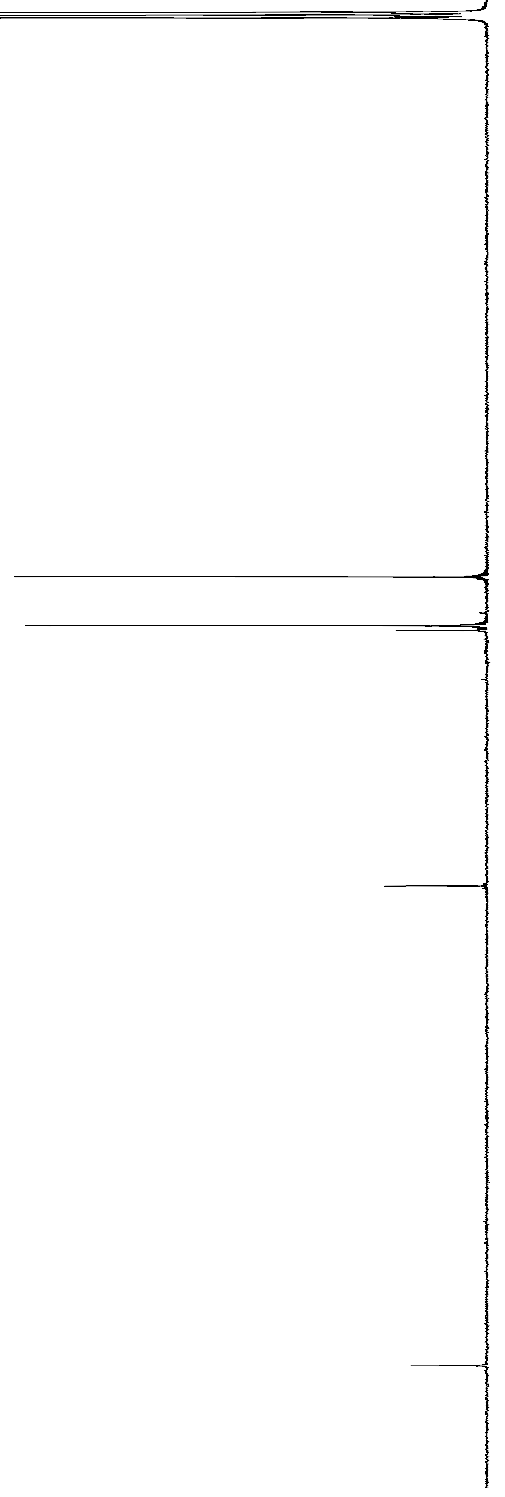

$\infty$ 


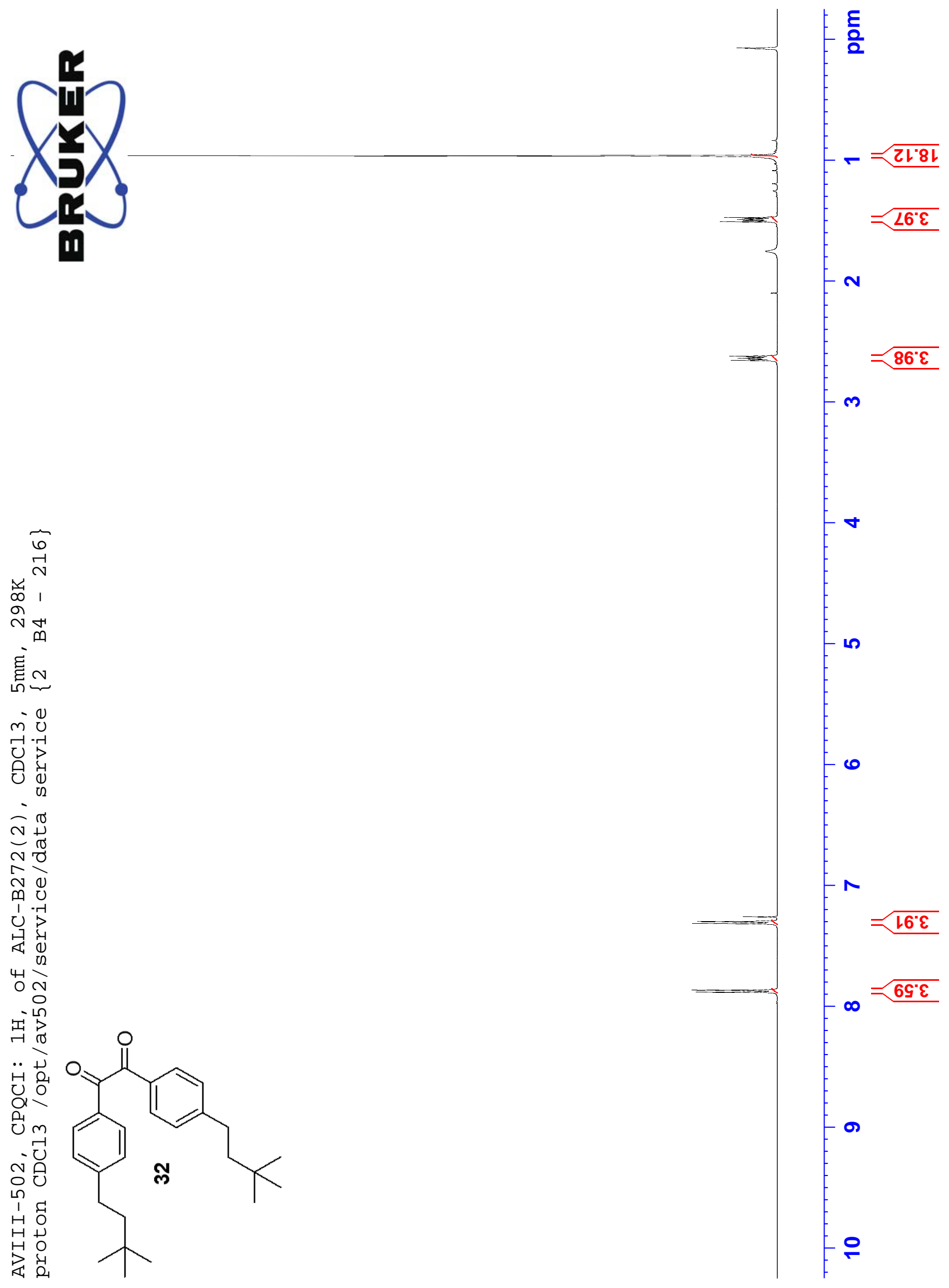


鄷

言

०乙 $6 乙$

०9. $\odot \varepsilon$

$99^{\circ}$ โ

E8' G†

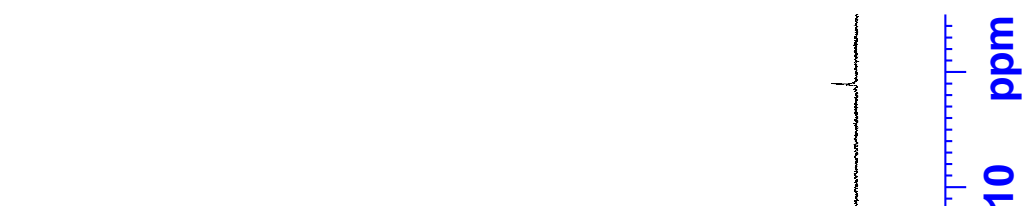

욱

우

요

움

우

8

$\stackrel{0}{1}$

$\infty$

×

N'

ह

हิ

लं

पु.

บे

- n

حิ



$N$ ত ZL. OEL

$\infty 0$

'ن

$\frac{1}{4} \frac{1}{d}$

$4 \stackrel{9}{5}$

पे 69 . TST
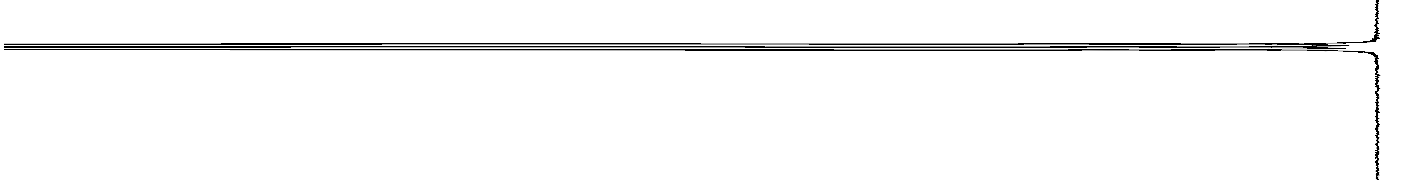

نิ

บำ

..

버응

넝

의

-

ำ

๑

1ㄷํㄴ

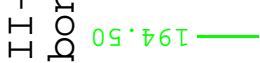

$5 \frac{1}{5}$
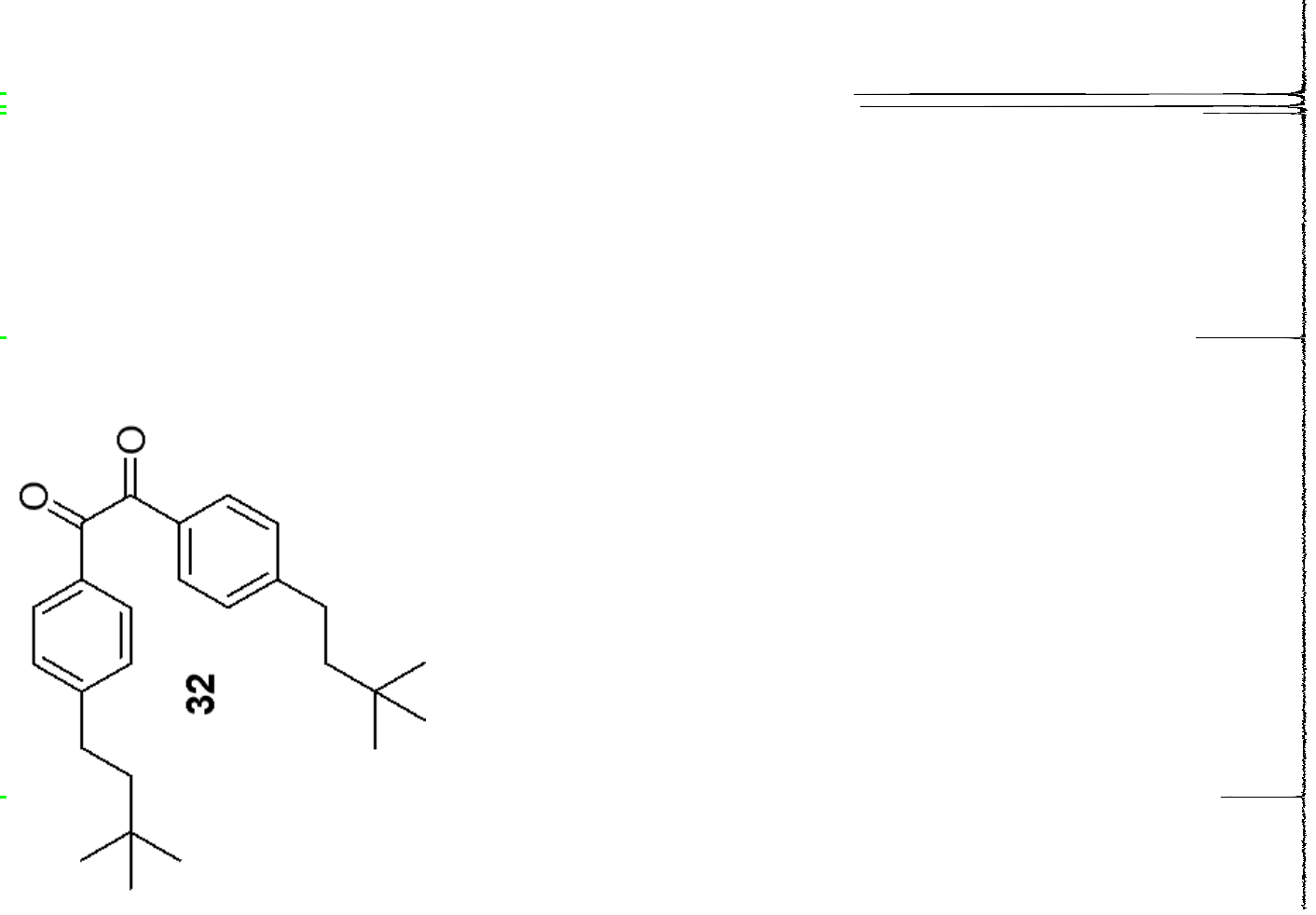

욱
울

- 윰

8

육

임

욱

옹 

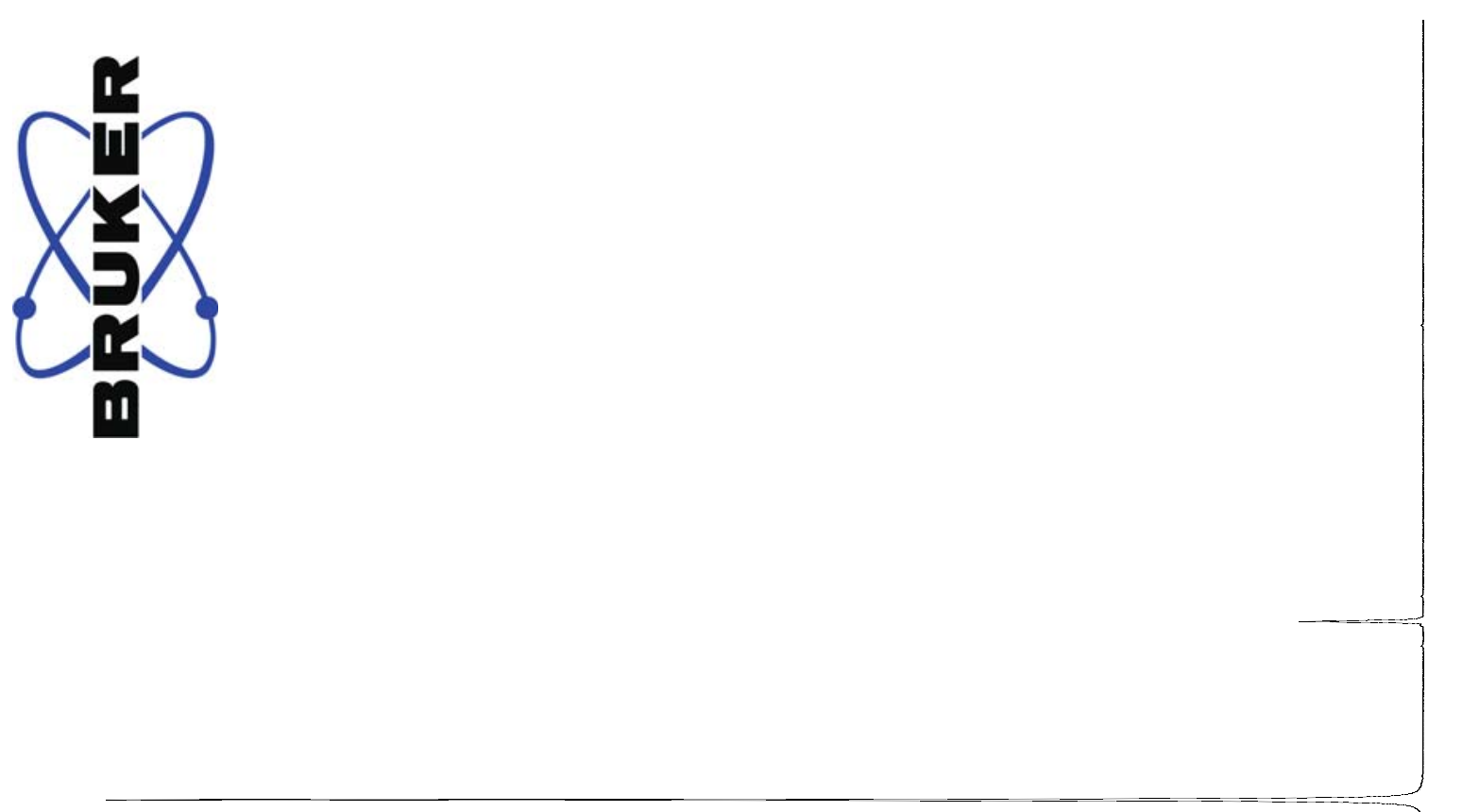

틍

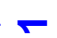

N

$\stackrel{\sim}{\sim}$

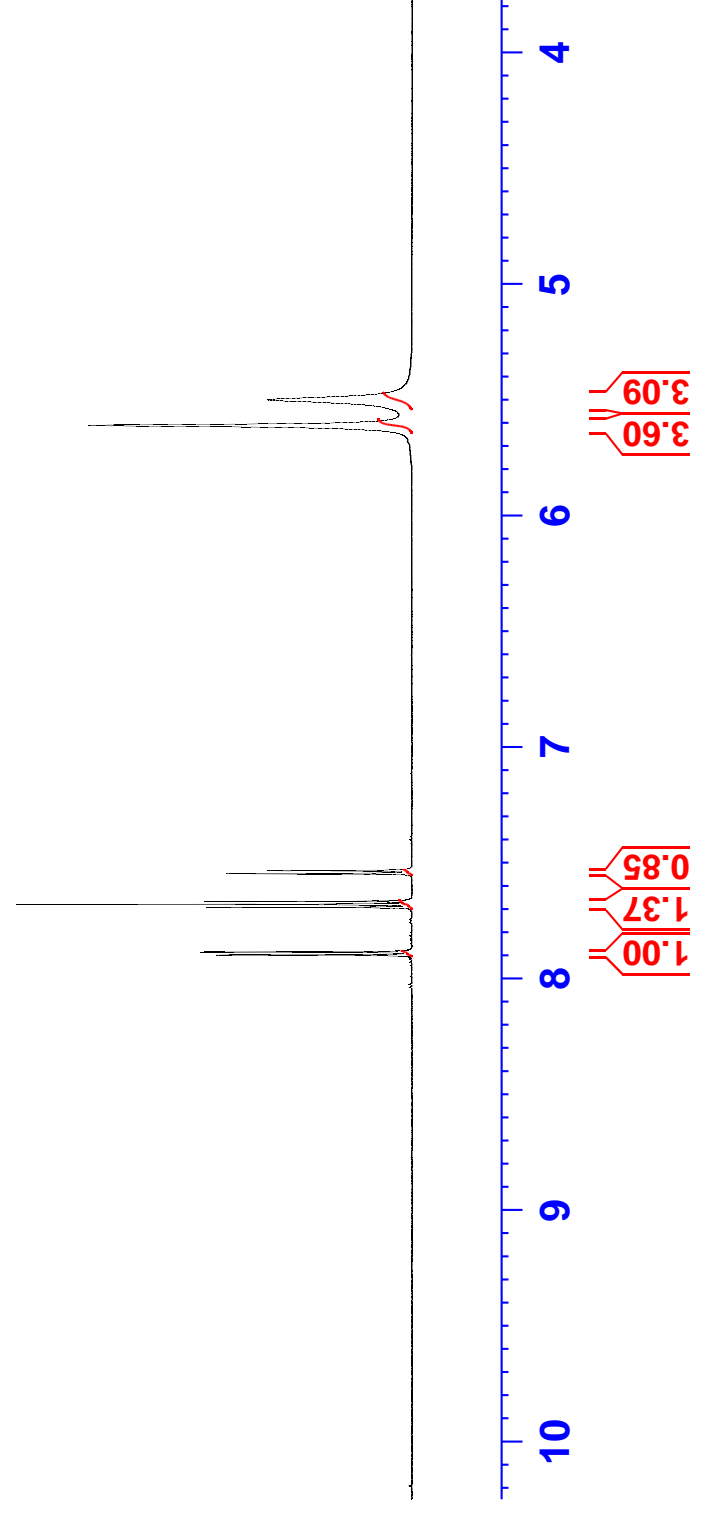

ํํำ

ஸे

$\sum_{0}^{\infty} \underbrace{N}$

ᄃ

ก气

\#

(1) 䒠

듵융

๙ิ ปे

ब्ड

덜가

ฮิ છ

이

지

능 응

저

4 응

工

I

नं

๑)

둥우

븜

¿

$\stackrel{N}{i} \quad{ }^{N}$
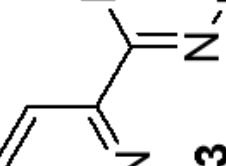

Z m

ตั 

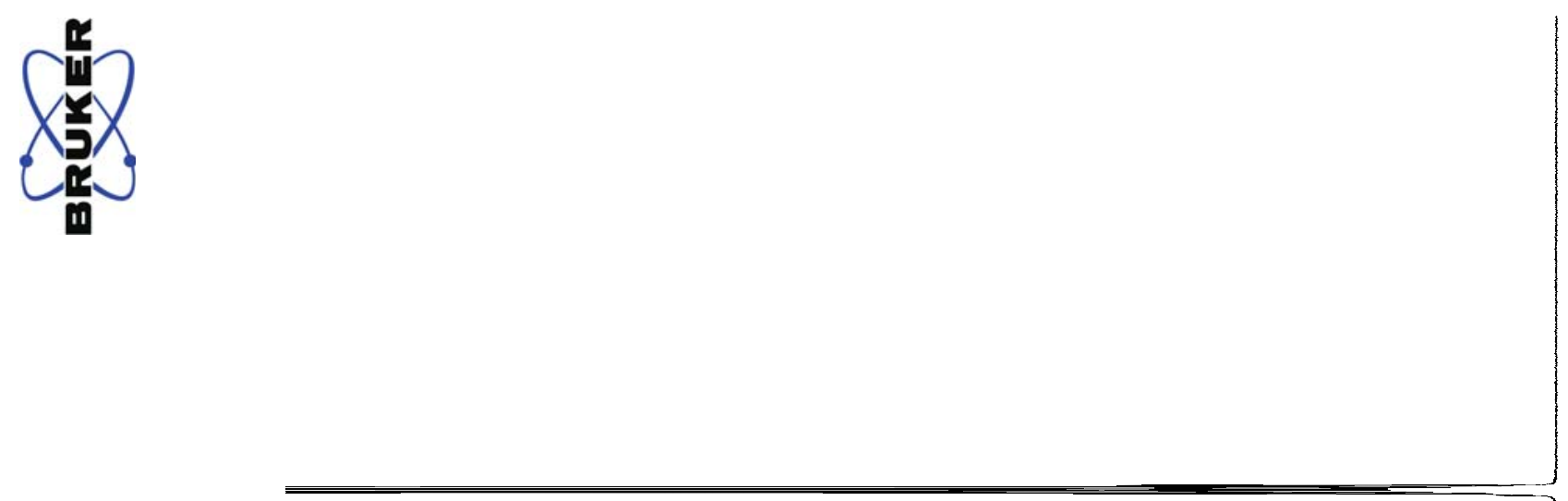

등 우

ㅇํ 요

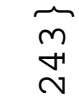

ل

.ᄃำ

\# 气

(1)

풍

읕즁 99.92 —

ஸे

过 हે

○ $\angle G^{\circ} 6 \varepsilon \tau \longrightarrow$

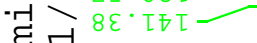

สে

ㄷํㅇ

N $\widetilde{\sigma} \odot \mathcal{E}^{* \varepsilon S T}$

ชে

능 뭉

포잉
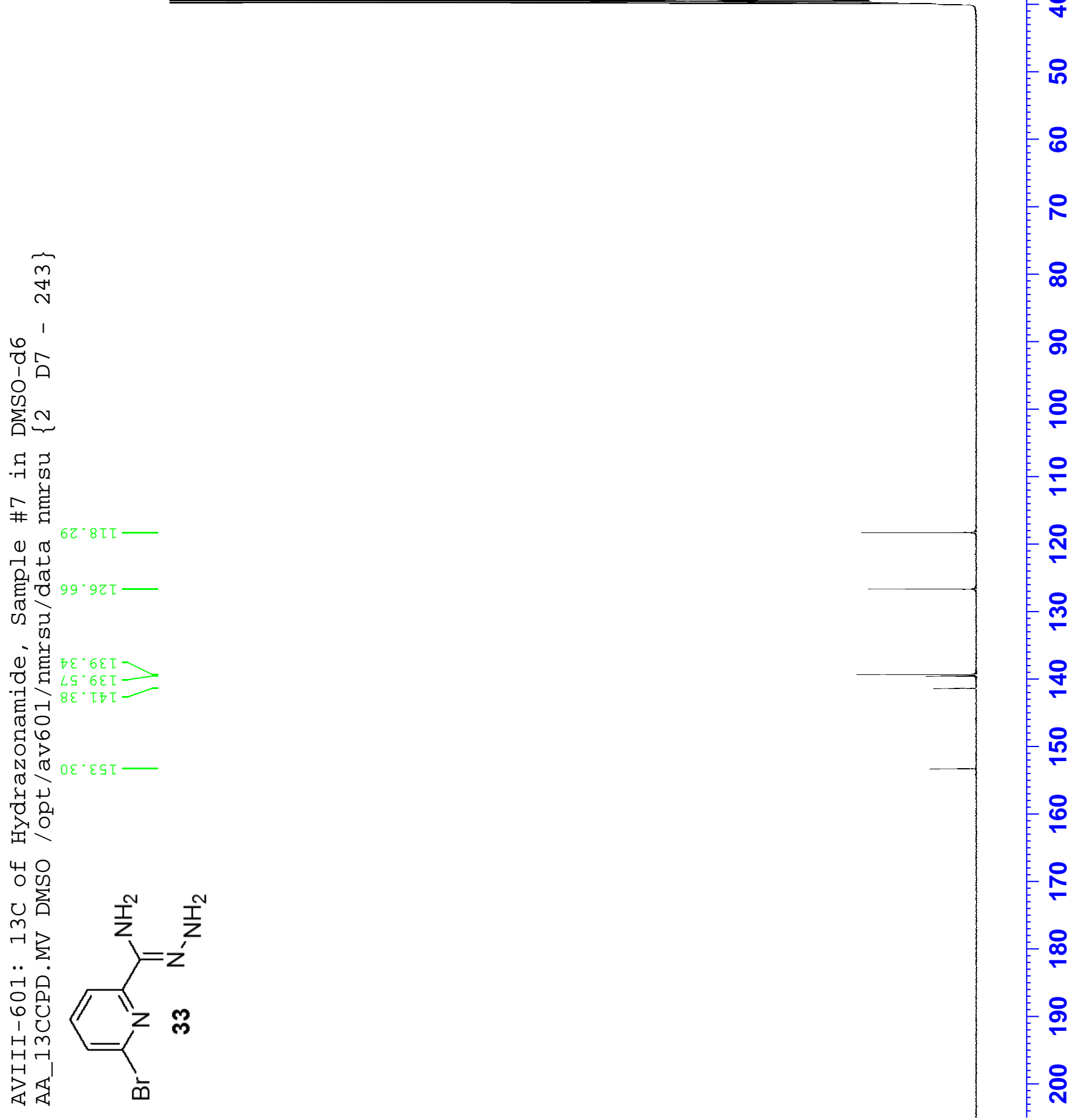

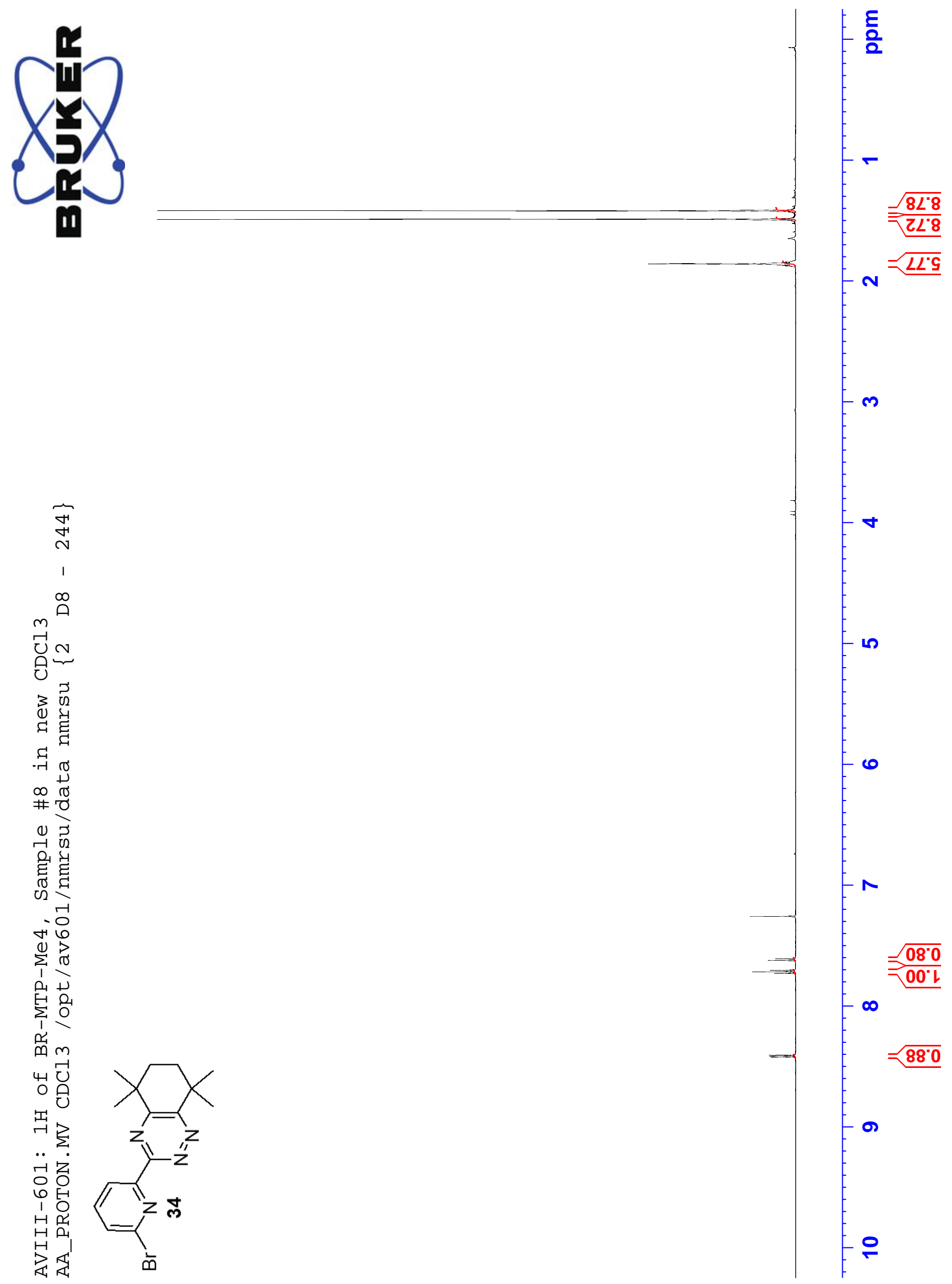
鄷

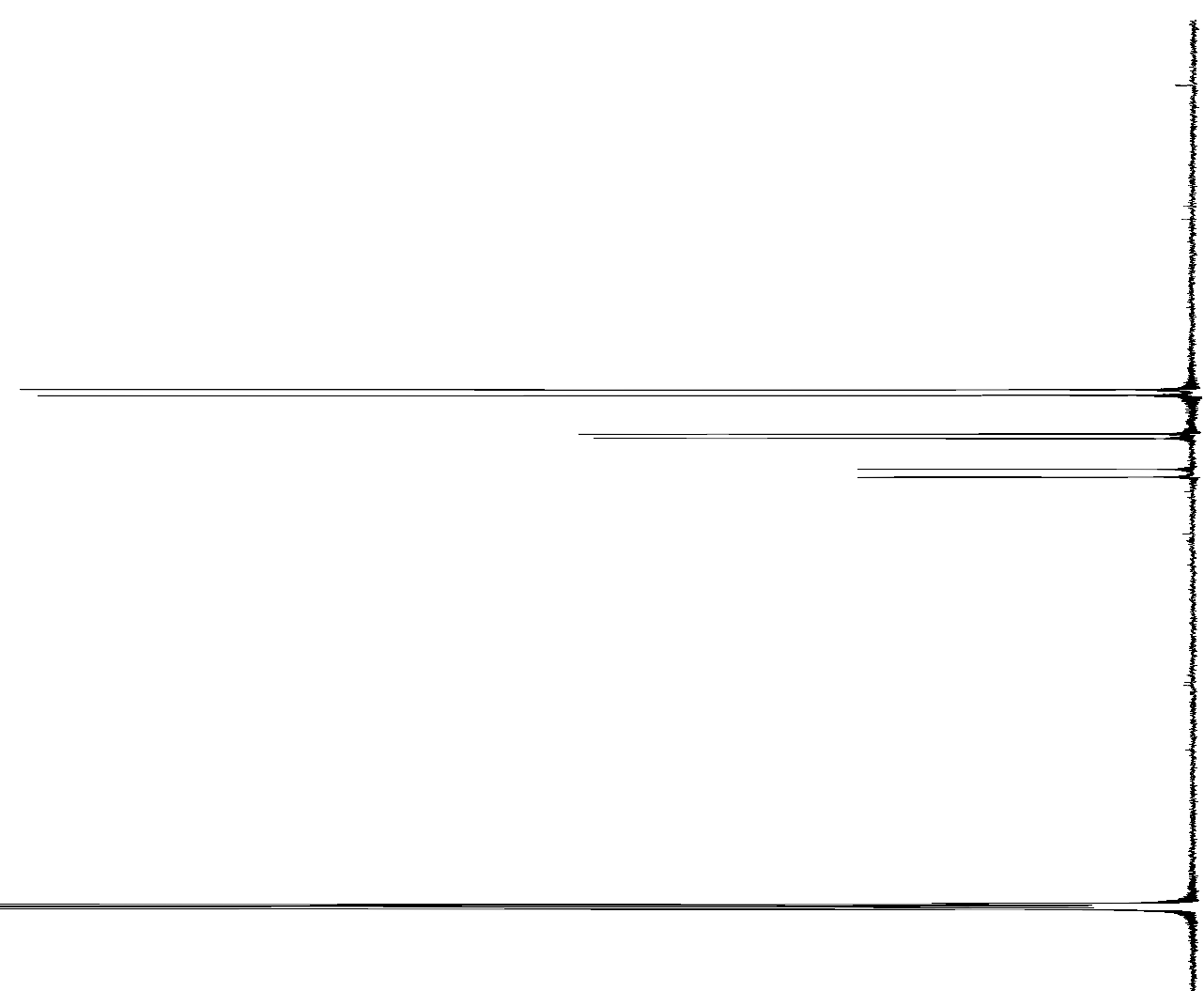

$\frac{\varepsilon}{2}$

$=$ 욱

요

$8[\cdot 62 \searrow$

$\angle 乙 \cdot \varepsilon \varepsilon$

$89^{\circ} \varepsilon \varepsilon$

$\varepsilon \mathcal{G}^{\prime} 9 \varepsilon$



'

3)

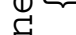

ᄃ जे

$\# \subseteq$

(1)

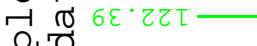

을임

๙

N हो

는

19

$\stackrel{ }{7}$

강

نำ

$\cup \widehat{O} 6 L$. 6GT

这 $8 \varepsilon^{\circ} \varepsilon 9 \tau$

4 党

บิ

m

..

겅응

60

$\mapsto \mathrm{m}$

답

$\gtreqless \frac{\square}{4}$
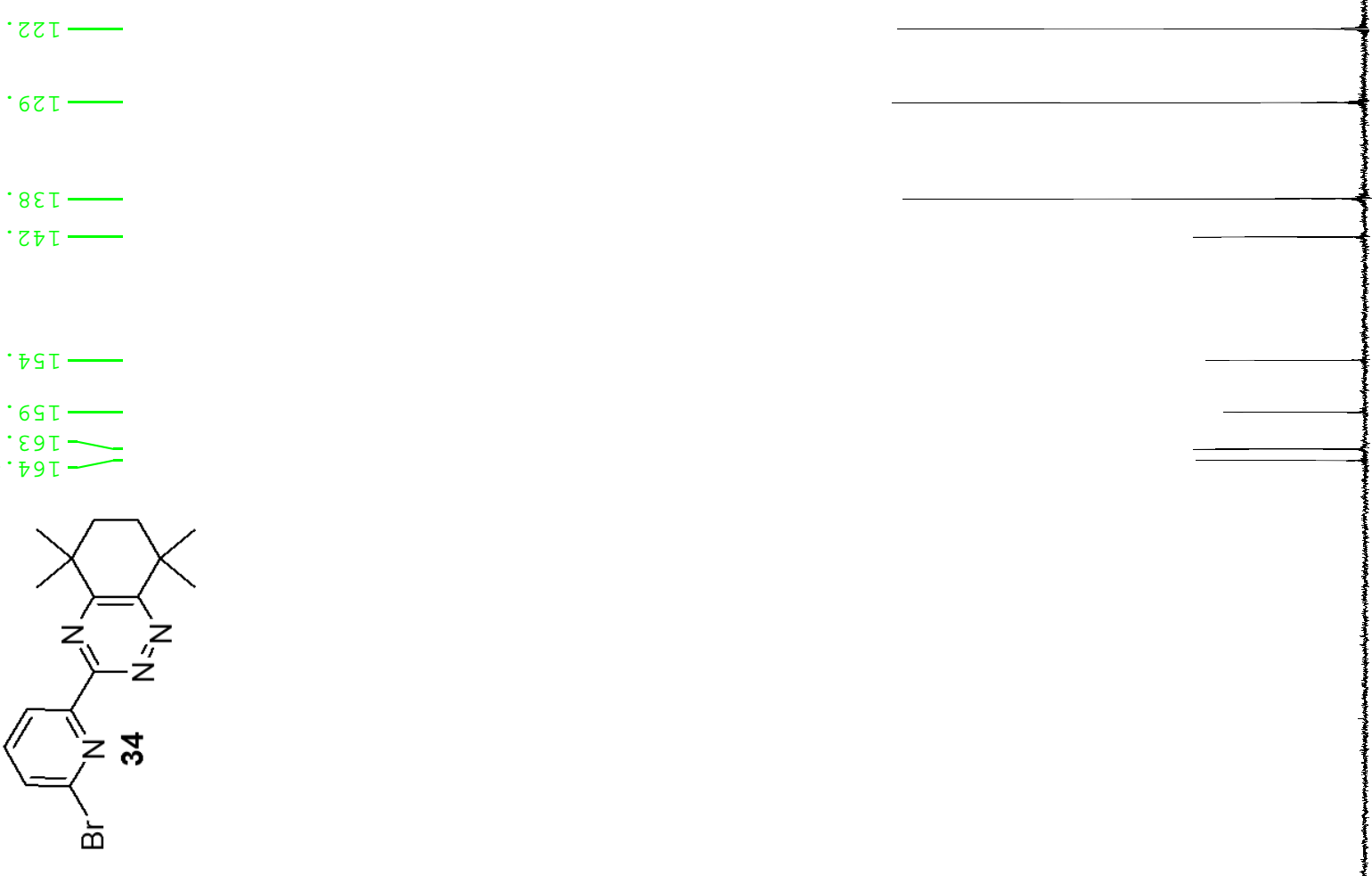
요 

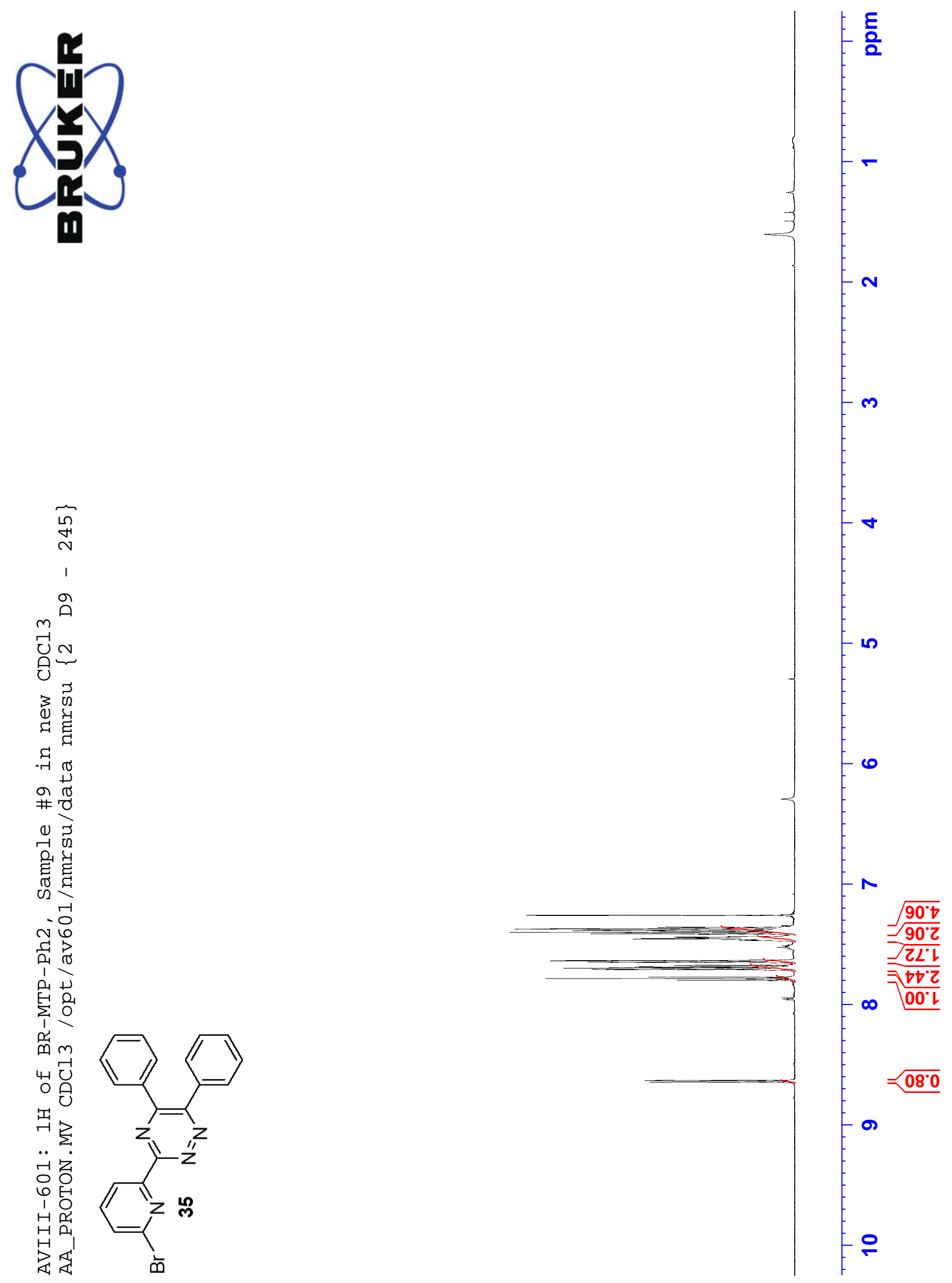


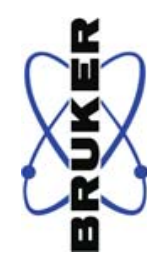

言 욱

오

우

우

웅

8

운

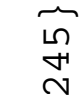

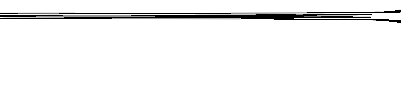

ल 1

거음

บ

3

过

ᄃ્ન

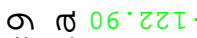

\#+ $\angle 9^{\prime} 8 Z T$

(1)

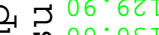

ป

๗ हो $\varepsilon 6^{\circ} \circ \varepsilon \tau$

$-\varepsilon \tau$ 'se

강.

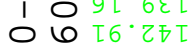

$\stackrel{\sim}{*}$

구응

के

ù 응

$\frac{1}{4}$

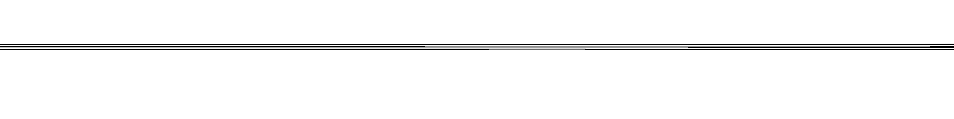

$\infty$

8

욱

국

욱

욤

옥

윰

웅

我

육

$\stackrel{\infty}{\infty}$

욤

오 

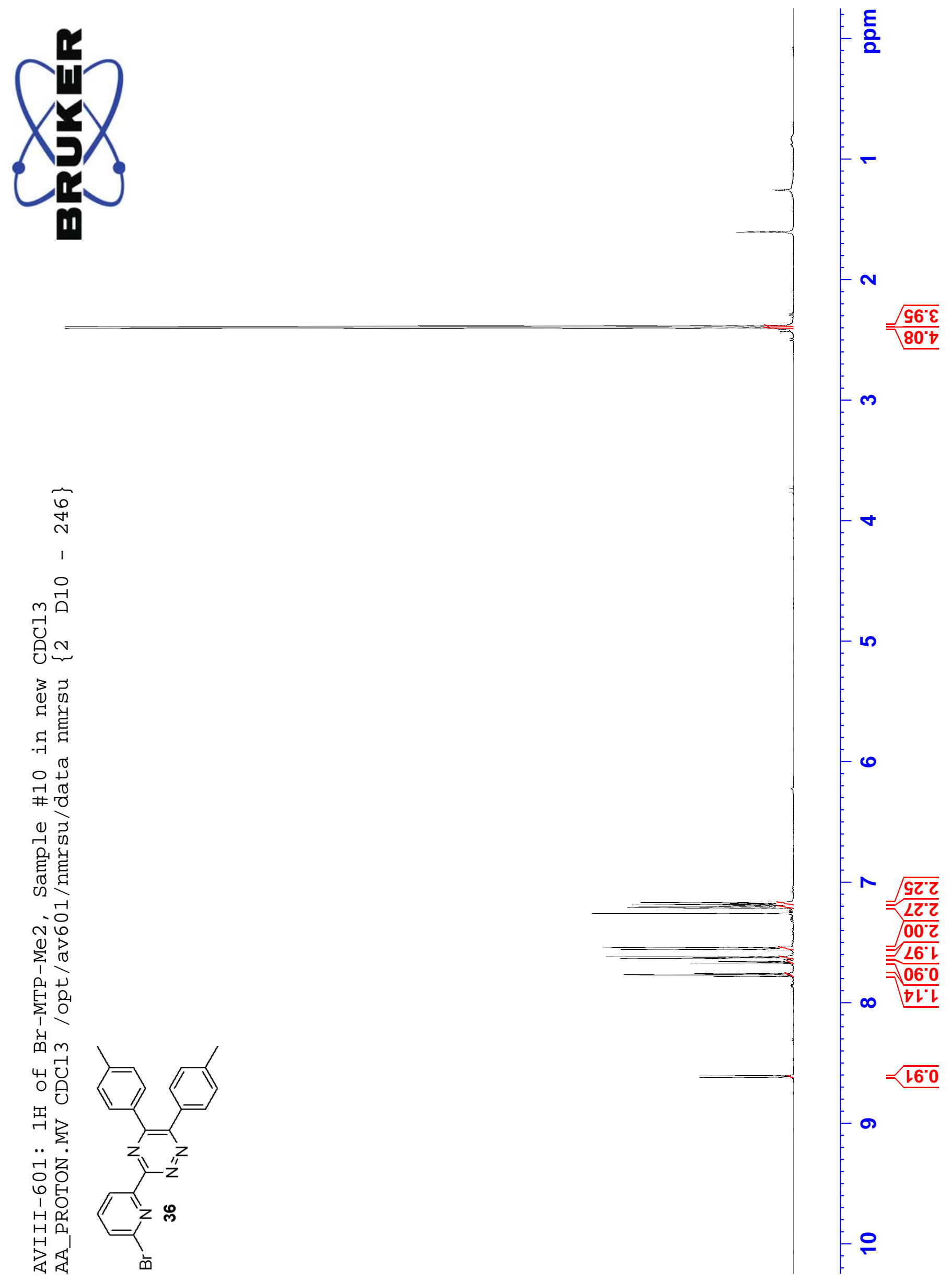


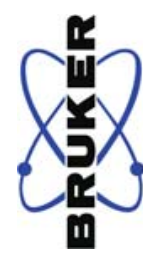

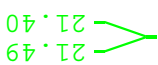

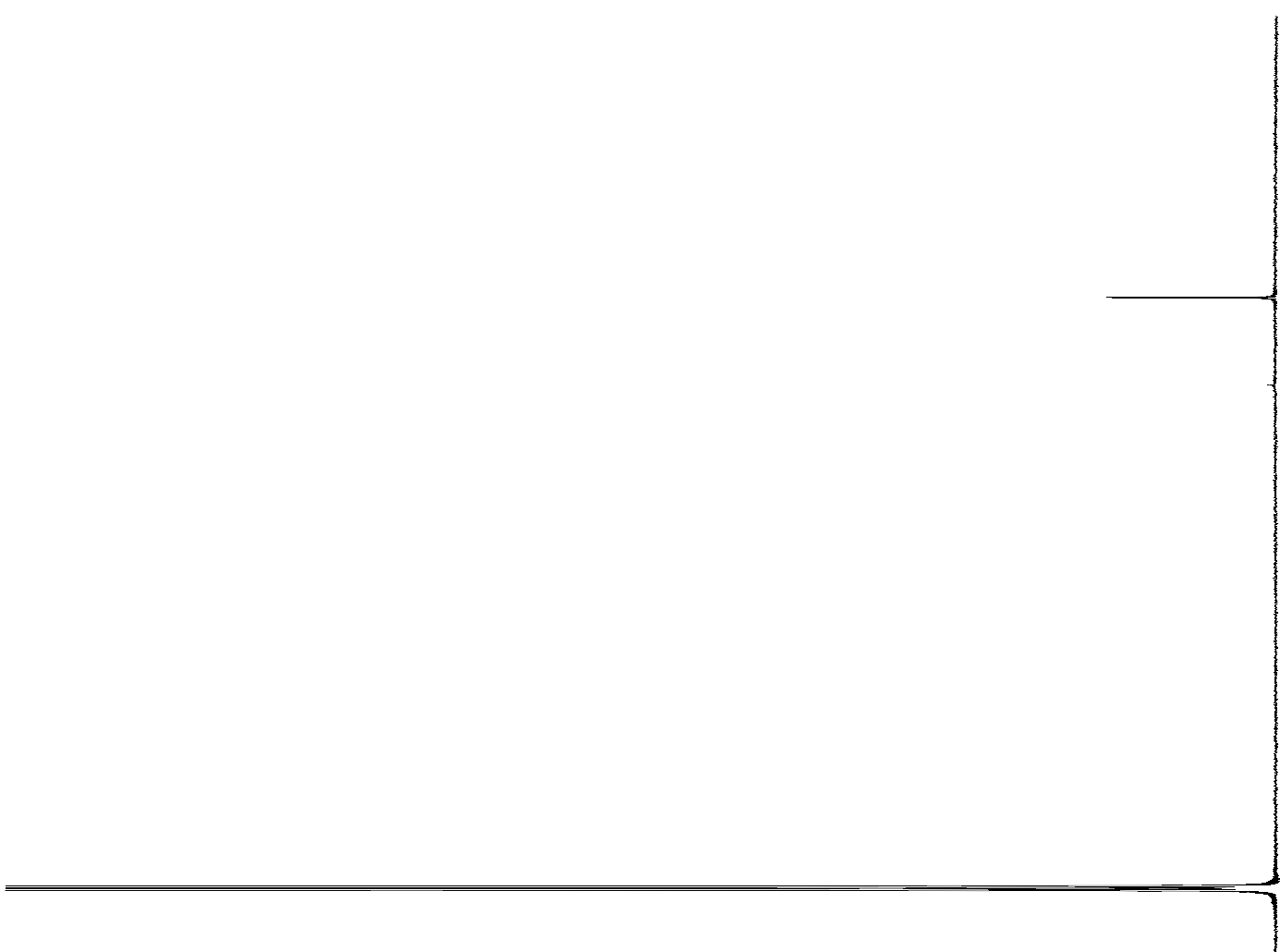



'

离 N

다 궁

를

\#

(1)

겅 중 6L'ZZL-

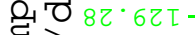

言

๗ ら $86^{\circ} 62 \tau$

हो z9.ZET

N

'

ल न्न

๑ $9 \nabla 8$ '

$\underset{7}{*}$

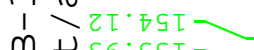

응

)

ष
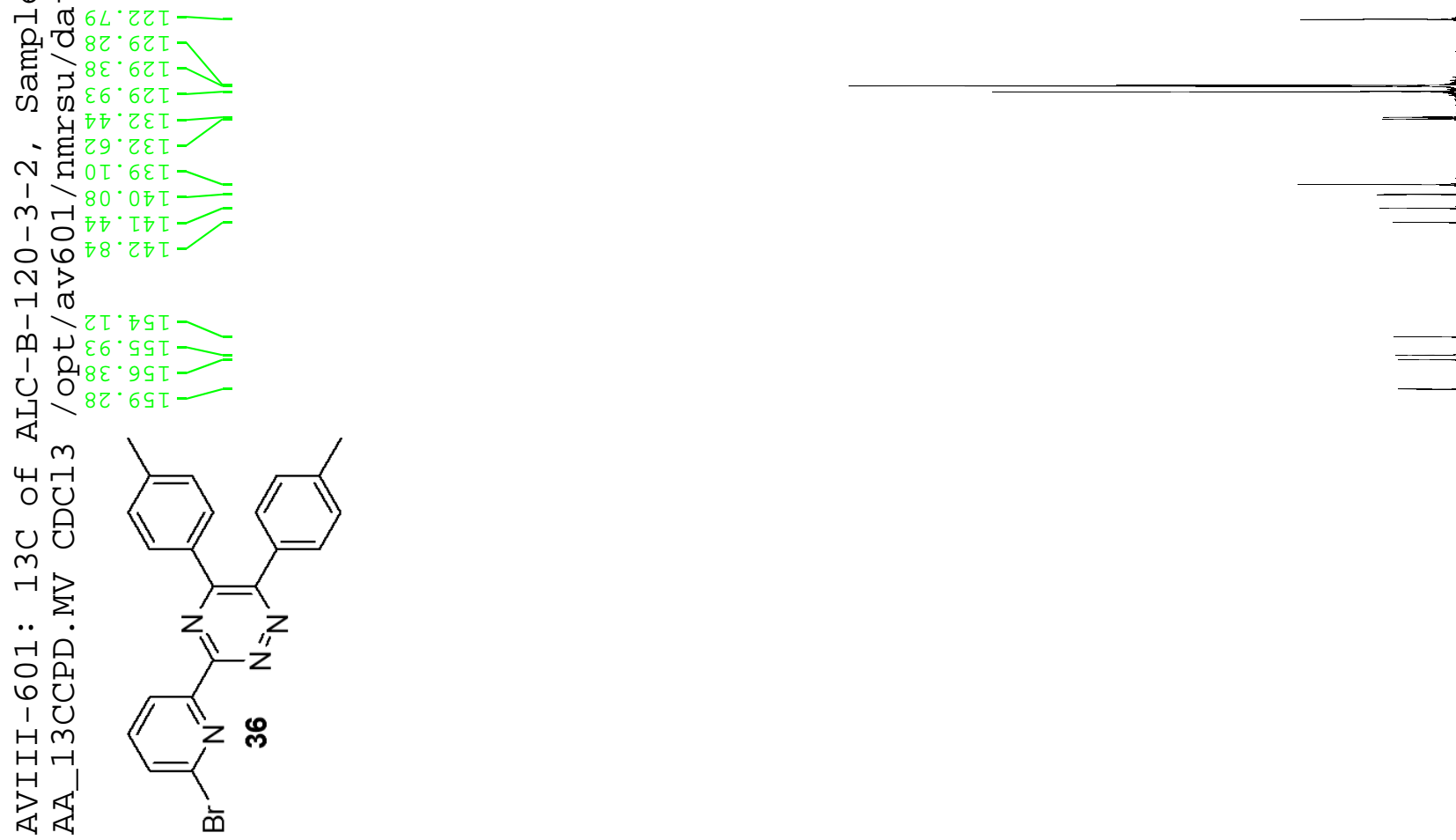

$\frac{\varepsilon}{2}$

욱

유

요

웅

운

8

운

$\infty$

ㅇ

:

욱

욱

욱

욤

욱

0

욱

익

옥

옹 

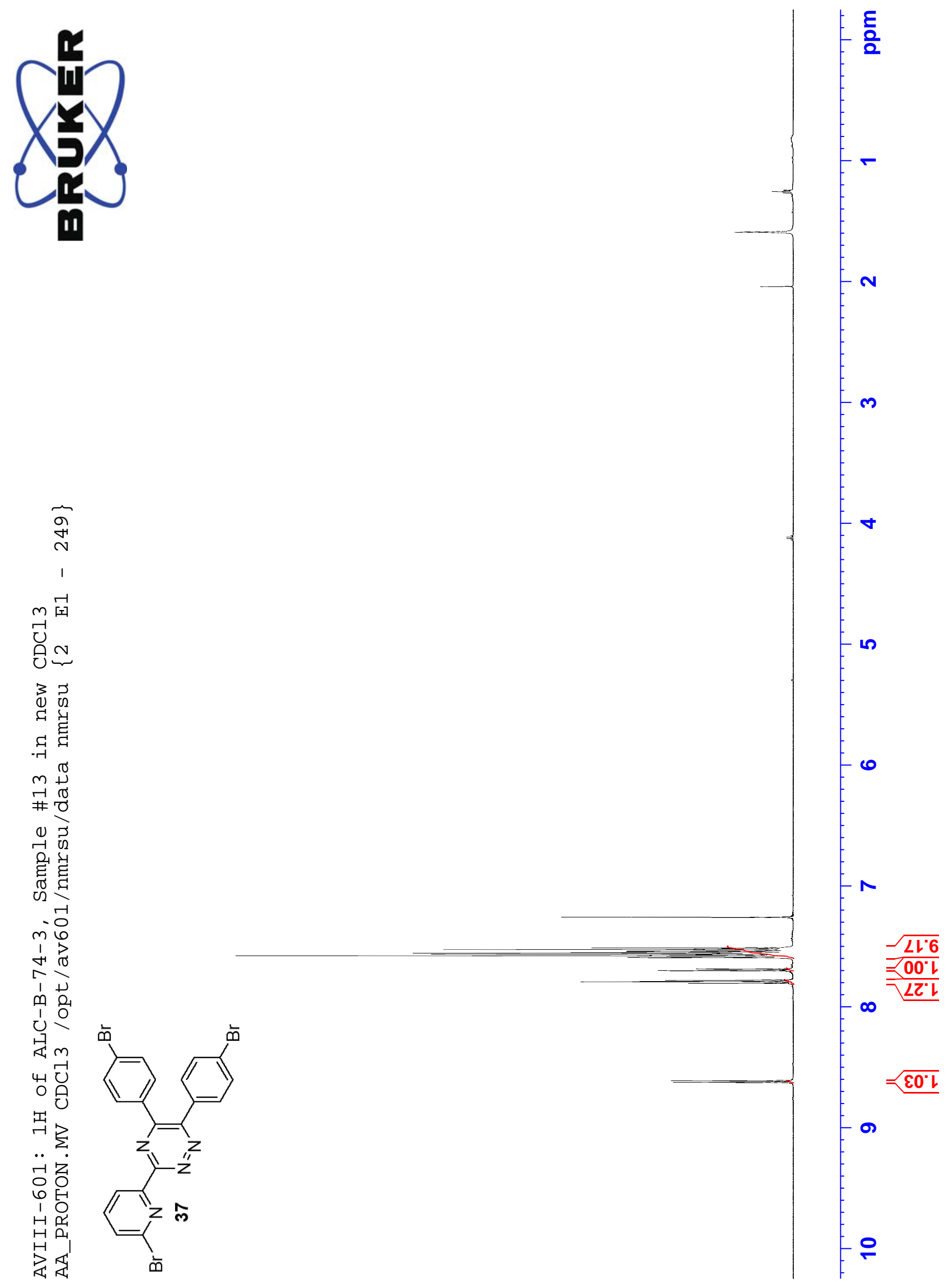


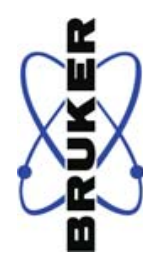

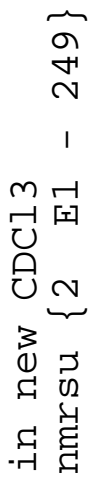

๓ $66^{\circ} 2 Z I$

\#

(1) $9 \varepsilon^{\circ}$.

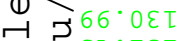

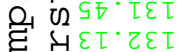

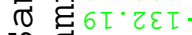

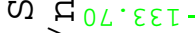

ग강.

लं

$\dot{N}$

ז

'่

' 응 LE' $99 \tau$

00
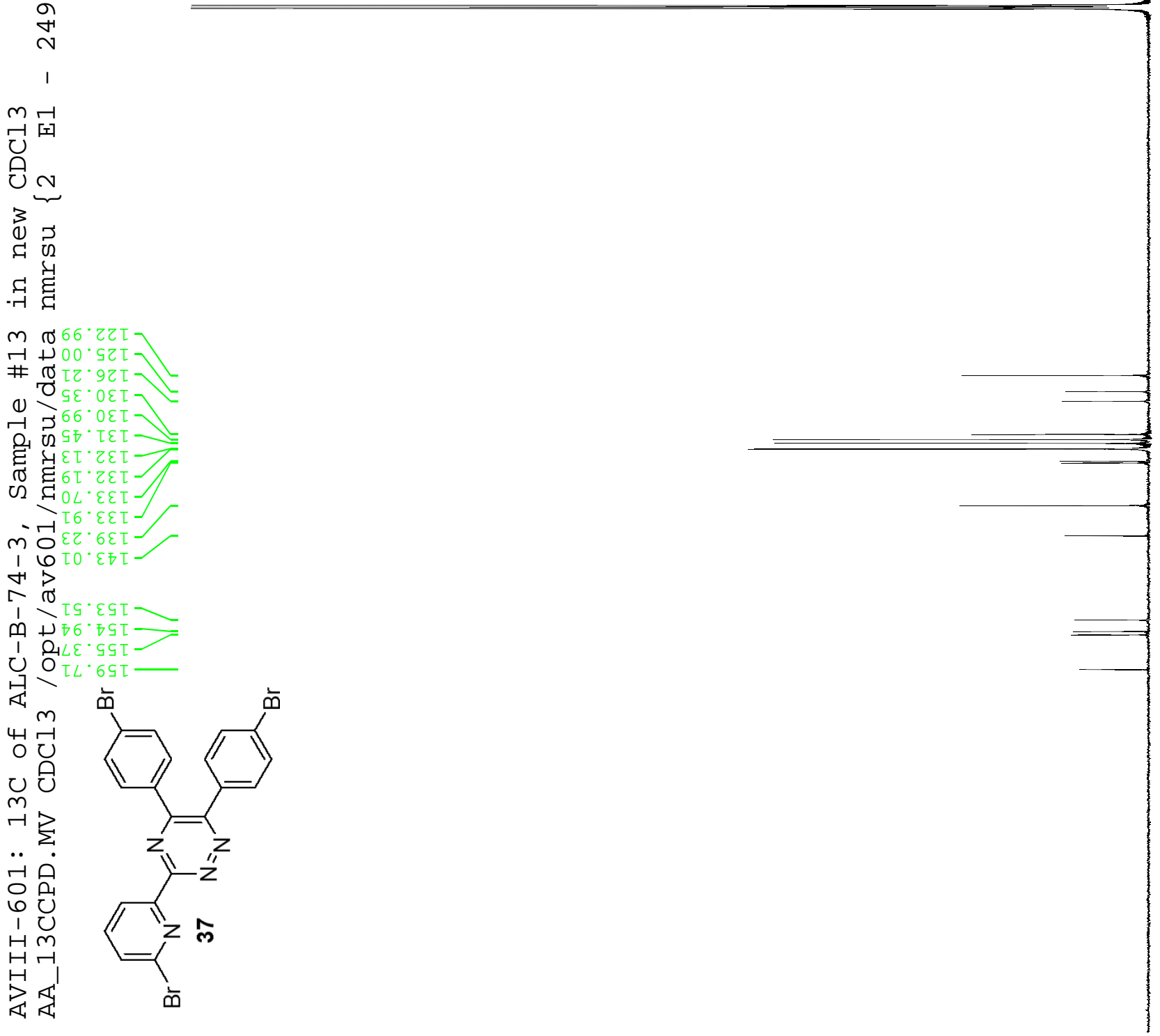

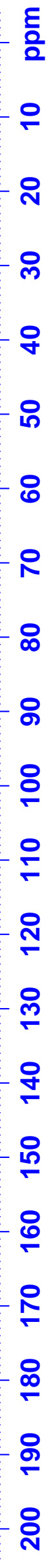


Compound [6]

Sample: ALC-B-188

4x Column: 2

RediSep Column: Silica $4 \mathrm{~g}$

Flow Rate: $18 \mathrm{ml} / \mathrm{min}$

Equilibration Volume: $33.6 \mathrm{ml}$

Initial Waste: $0.0 \mathrm{ml}$

Air Purge: 10.0 min

Solvent: A1 hexane

Solvent: B1 ethyl acetate

\section{Rf 200}

Peak Tube Volume: Max.

Non-Peak Tube Volume: Max.

Loading Type: Solid

Wavelength 1 (red): 254nm

Peak Width: 30 sec

Threshold: 0.20 AU

Wavelength 2 (purple): 280nm
Wednesday 09 September 2015 02:03PM

All Wavelength (orange): 200nm - 360nm

Peak Width: 30 sec

Threshold: 0.20 AU

Run Notes:

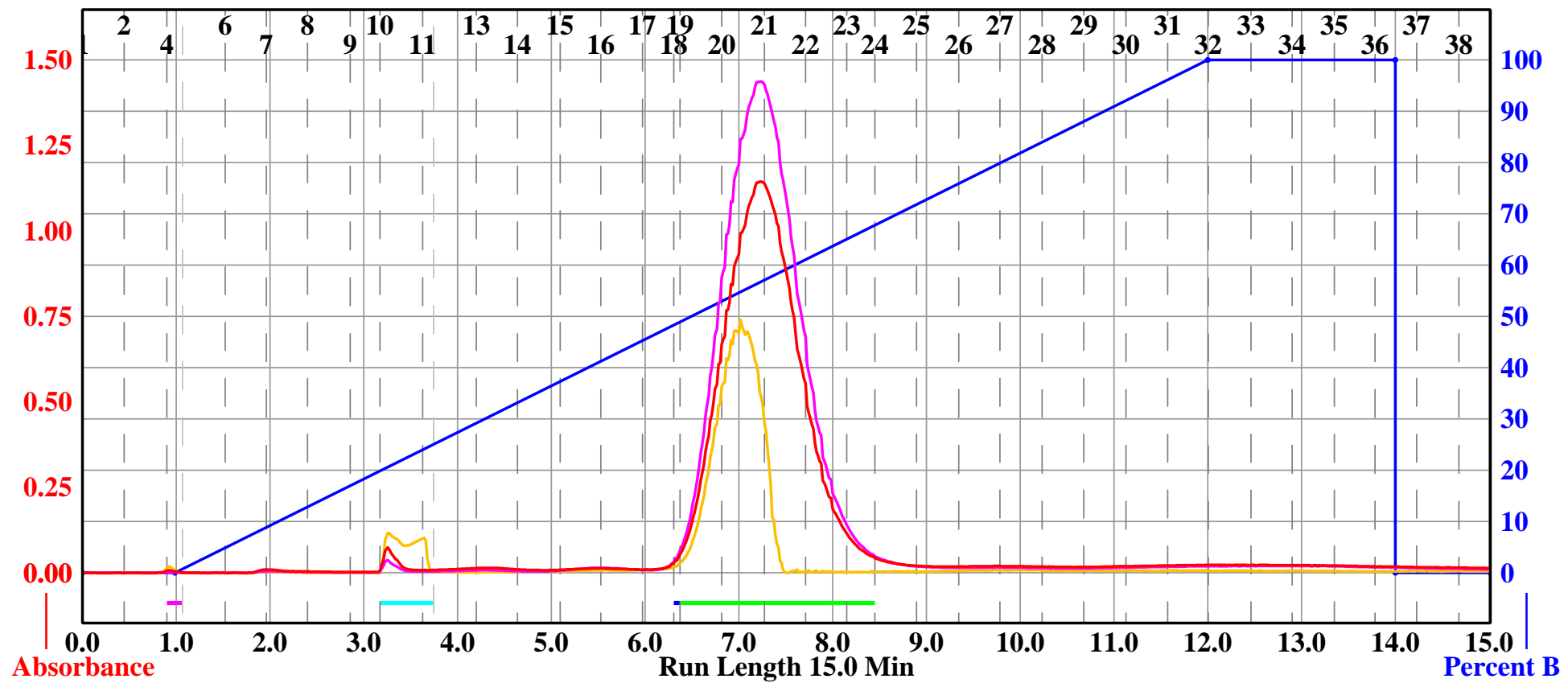

Rack A

\begin{tabular}{|c|c|c|c|c|c|}
\hline 8 & (107) & 1 (106 & 105 & (104 & (103 \\
\hline 27 & $\mathscr{B}$ & (9) & 100 & (1) & 102 \\
\hline Vf & 9 & (A) & 19 & (2) & (2) \\
\hline 3 & 8 & 87 & 88 & (9) & (2) \\
\hline 89 & 8 & 82 & 80 & 80 & (19) \\
\hline 13 & (8) & (15) & (ש) & (17) & 18 \\
\hline (2) & (1) & (10) & (9) & 68 & (67) \\
\hline (1) & (2) & (3) & (4) & (6) & ஏ \\
\hline (10) & 59 & 68 & 57 & ஒ & 57 \\
\hline (4) & (D) & (D) & 52 & 53 & 59 \\
\hline 48 & (47) & (46) & (45) & (4) & 43 \\
\hline (37) & B8 & (99) & (4) & (47) & (42) \\
\hline bd & (3) & B & B3 & (32) & (1) \\
\hline 25 & ש & (28) & 8 & (29) & (10) \\
\hline (2) & 23 & 22 & (D) & (21) & (1) \\
\hline (1) & (1) & (15) & (16) & (17) & 8 \\
\hline (12) & (1) & (1) & (2) & (8) & (7) \\
\hline (1) & (2) & (3) & (4) & (5) & (6) \\
\hline
\end{tabular}

$13 \mathrm{~mm} \times 100 \mathrm{~mm}$ Tubes

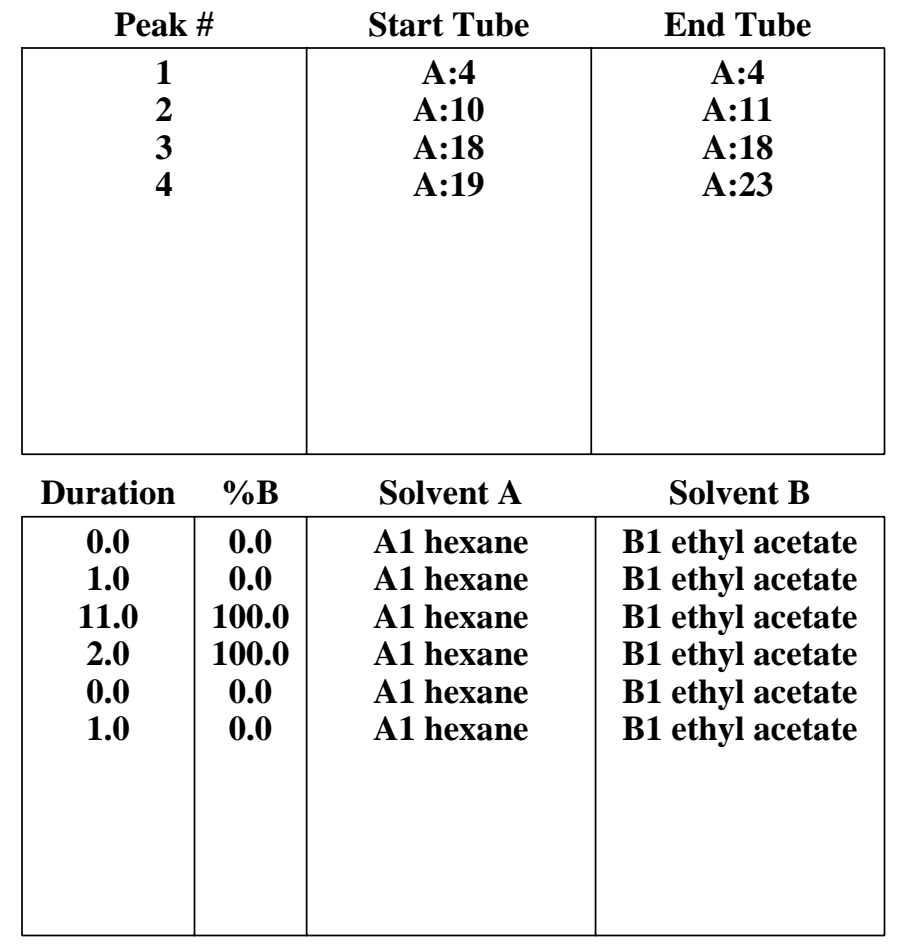


Compound [11]

Sample: ALC-B-216-2

4x Column: 3

RediSep Column: Silica 4g

Flow Rate: $18 \mathrm{ml} / \mathrm{min}$

Equilibration Volume: $33.6 \mathrm{ml}$

Initial Waste: $0.0 \mathrm{ml}$

Air Purge: 10.0 min

Solvent: A1 hexane

Solvent: B1 ethyl acetate

\section{Rf 200}

Peak Tube Volume: Max.

Non-Peak Tube Volume: Max.

Loading Type: Solid

Wavelength 1 (red): 254nm

Peak Width: 30 sec

Threshold: 0.20 AU

Wavelength 2 (purple): 280nm
Thursday 17 September 2015 01:09PM

All Wavelength (orange): $200 \mathrm{~nm}-360 \mathrm{~nm}$

Peak Width: $30 \mathrm{sec}$

Threshold: 0.20 AU

Run Notes:

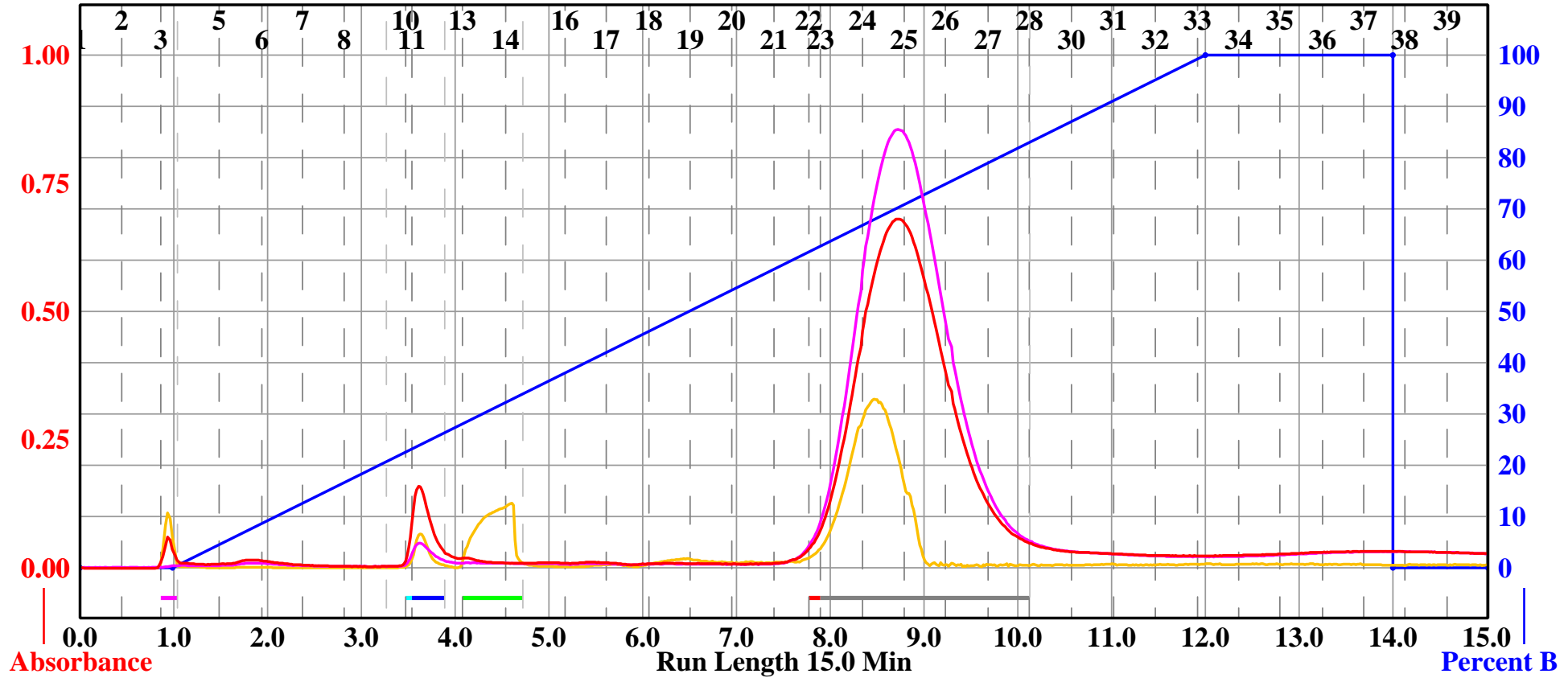

Rack A

Peak \#

Start Tube

End Tube

\begin{tabular}{|c|c|c|c|c|c|}
\hline & (107 & 1 (106 & 105 & (104 & (10) \\
\hline 27 & (8) & (9) & (100) & (1)1 & (10) \\
\hline$\sqrt{6}$ & (19 & (9) & (3) & (2) & (2) \\
\hline 3 & 8 & 87 & 88 & 89 & (2) \\
\hline 84 & 83 & 82 & 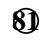 & 80 & (7) \\
\hline 13 & (18) & (28) & (16) & (27) & 18 \\
\hline 12 & (11) & (11) & (9) & 8 & (7) \\
\hline (1) & (2) & (3) & (4) & 6 & ஆ \\
\hline (10) & 59 & 68 & 57 & ஜ & 59 \\
\hline 49 & (D) & (D) & (52) & 53 & 67 \\
\hline 48 & (47) & (46) & 45 & (4) & 43 \\
\hline (37) & BB & (39) & (41) & (47) & (42) \\
\hline (10) & (35 & (3) & (3) & (12) & (10) \\
\hline 25 & ש & 82 & 8 & 29 & (B) \\
\hline (2) & 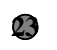 & 2 & (2) & (2) & (19 \\
\hline (1) & (1) & (15) & (16) & (17) & (18) \\
\hline (12) & 0 & (11) & (2) & (8) & (7) \\
\hline (1) & (2) & (3) & (4) & (5) & (6) \\
\hline
\end{tabular}

$13 \mathrm{~mm} \times 100 \mathrm{~mm}$ Tubes

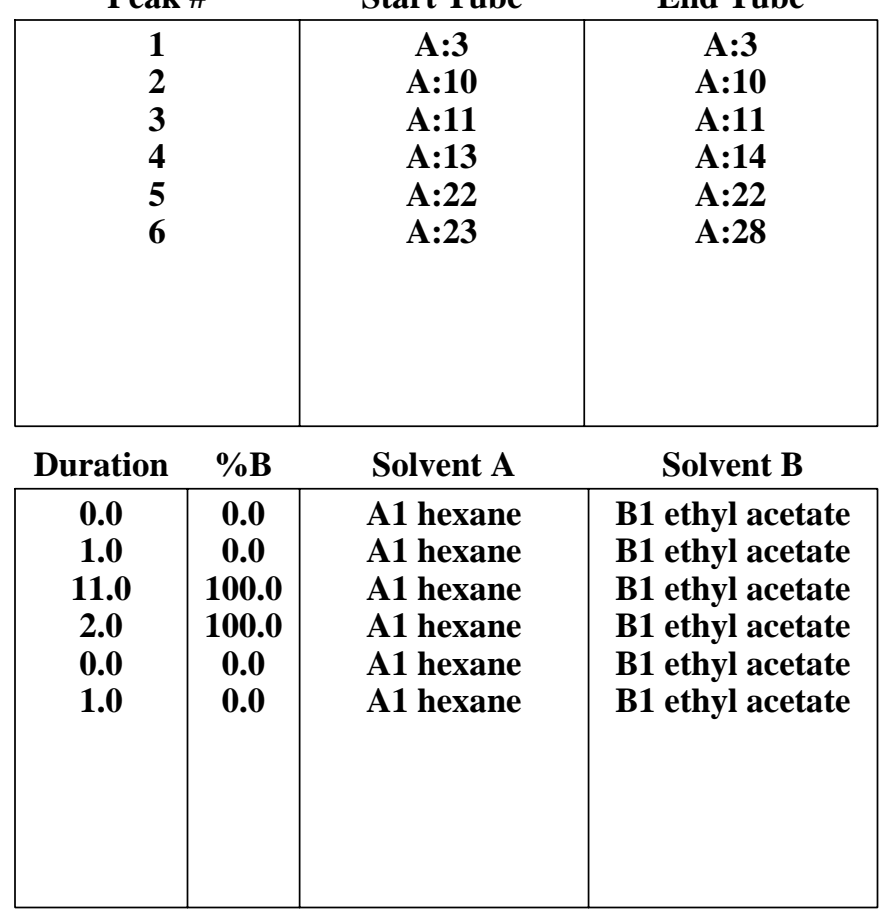


4x Column: 2

RediSep Column: Silica 4g

Flow Rate: $18 \mathrm{ml} / \mathrm{min}$

Equilibration Volume: $33.6 \mathrm{ml}$

Initial Waste: $0.0 \mathrm{ml}$

Air Purge: $10.0 \mathrm{~min}$

Solvent: A1 hexane

Solvent: B1 ethyl acetate
Peak Tube Volume: Max.

Non-Peak Tube Volume: Max.

Loading Type: Solid

Wavelength 1 (red): 254nm

Peak Width: $30 \mathrm{sec}$

Threshold: 0.20 AU

Wavelength 2 (purple): $280 \mathrm{~nm}$
All Wavelength (orange): 200nm - 360nm

Peak Width: 30 sec

Threshold: 0.20 AU

Run Notes:

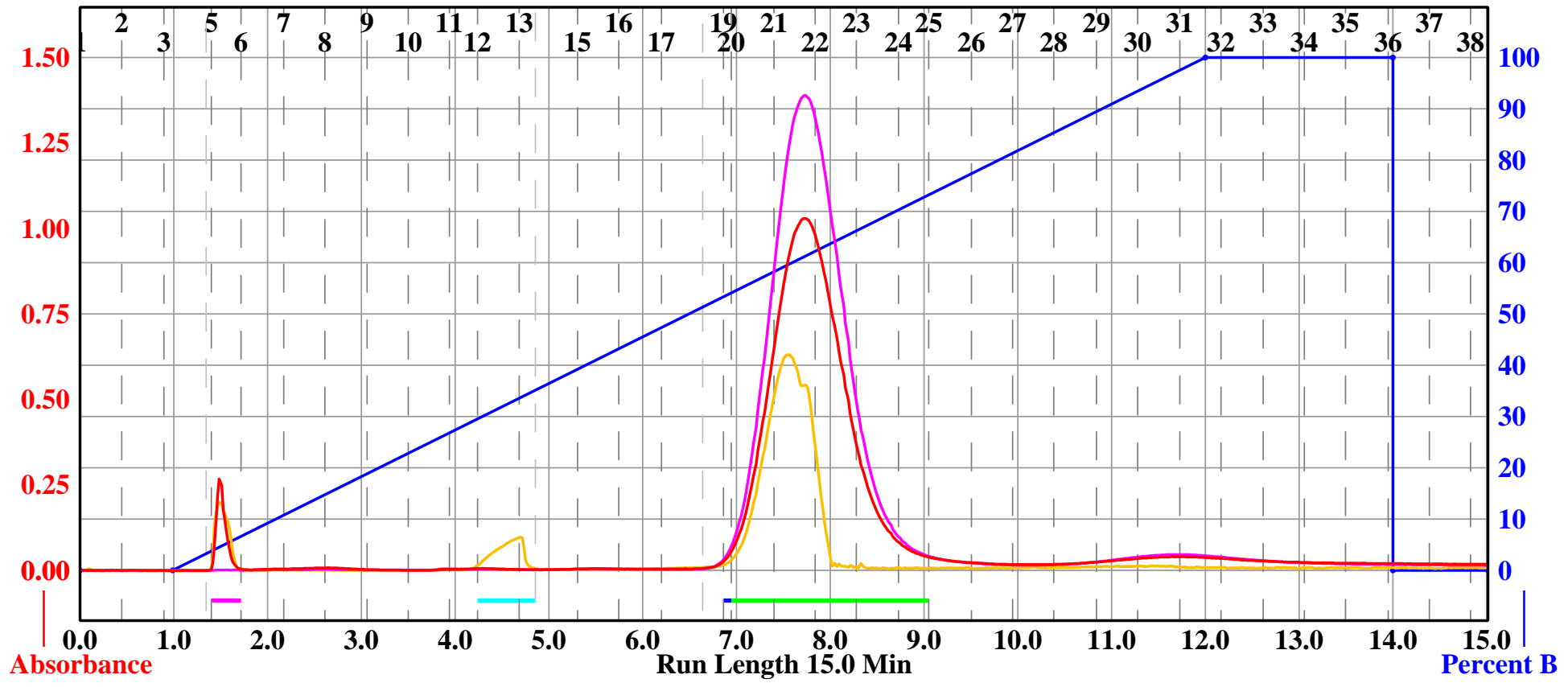

Rack A

\begin{tabular}{|c|c|c|c|c|c|}
\hline 08 & (107) & 106 & 105 & (104 & (10) \\
\hline 87 & Q8 & (9) & (100 & (1) & $1(102$ \\
\hline fd & (2) & 9 & $\mathscr{Q}$ & 22 & (D) \\
\hline 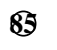 & 8 & 87 & 88 & 89 & VD \\
\hline 39 & 83 & 82 & 80 & 80 & (19) \\
\hline 33 & 18 & (2) & (8) & (17) & 18 \\
\hline 12 & (1) & (2) & (9) & 6 & (67) \\
\hline (1) & (2) & (3) & (4) & (6) & 6 \\
\hline (10) & 59 & $\mathfrak{6 8}$ & 57 & ஏd & 57 \\
\hline (19) & 50 & (1) & 52 & 33 & 59 \\
\hline 48 & (47) & (4) & (45) & (4) & (4) \\
\hline (37) & B8 & (39 & (41) & (4) & (42) \\
\hline (B) & BS & (3) & B3 & (32) & (1) \\
\hline (3) & ש2 & ed & 8 & (29) & (10) \\
\hline 29 & 3 & (2) & (D) & (21) & D \\
\hline (13) & $\mathbb{1}$ & (15) & (16) & (17) & $\mathbb{1 8}$ \\
\hline (12) & (11) & (11) & (D) & (8) & (7) \\
\hline (1) & (2) & (3) & (4) & (5) & (6) \\
\hline
\end{tabular}

$13 \mathrm{~mm} \times 100 \mathrm{~mm}$ Tubes

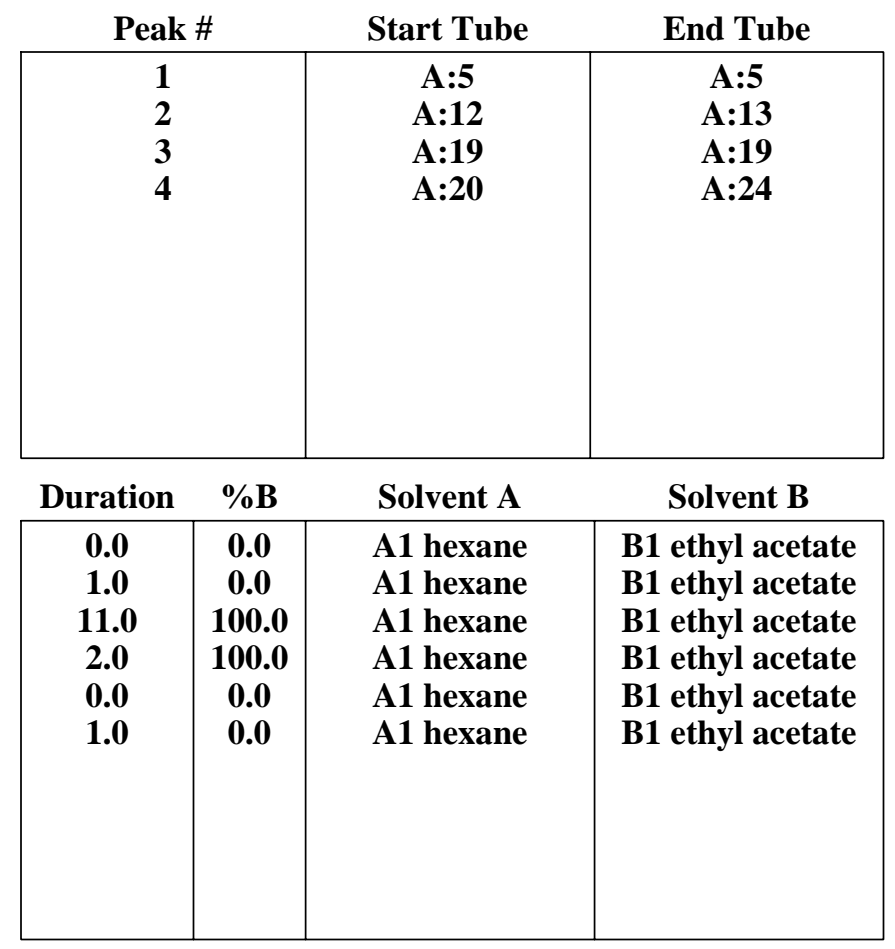


4x Column: 2

RediSep Column: Silica 4g

Flow Rate: $18 \mathrm{ml} / \mathrm{min}$

Equilibration Volume: $33.6 \mathrm{ml}$

Initial Waste: $0.0 \mathrm{ml}$

Air Purge: 10.0 min

Solvent: A1 hexane

Solvent: B1 ethyl acetate
Peak Tube Volume: Max.

Non-Peak Tube Volume: Max.

Loading Type: Solid

Wavelength 1 (red): 254nm

Peak Width: 30 sec

Threshold: 0.20 AU

Wavelength 2 (purple): $280 \mathrm{~nm}$
All Wavelength (orange): $200 \mathrm{~nm}-360 \mathrm{~nm}$

Peak Width: 30 sec

Threshold: 0.20 AU

Run Notes:

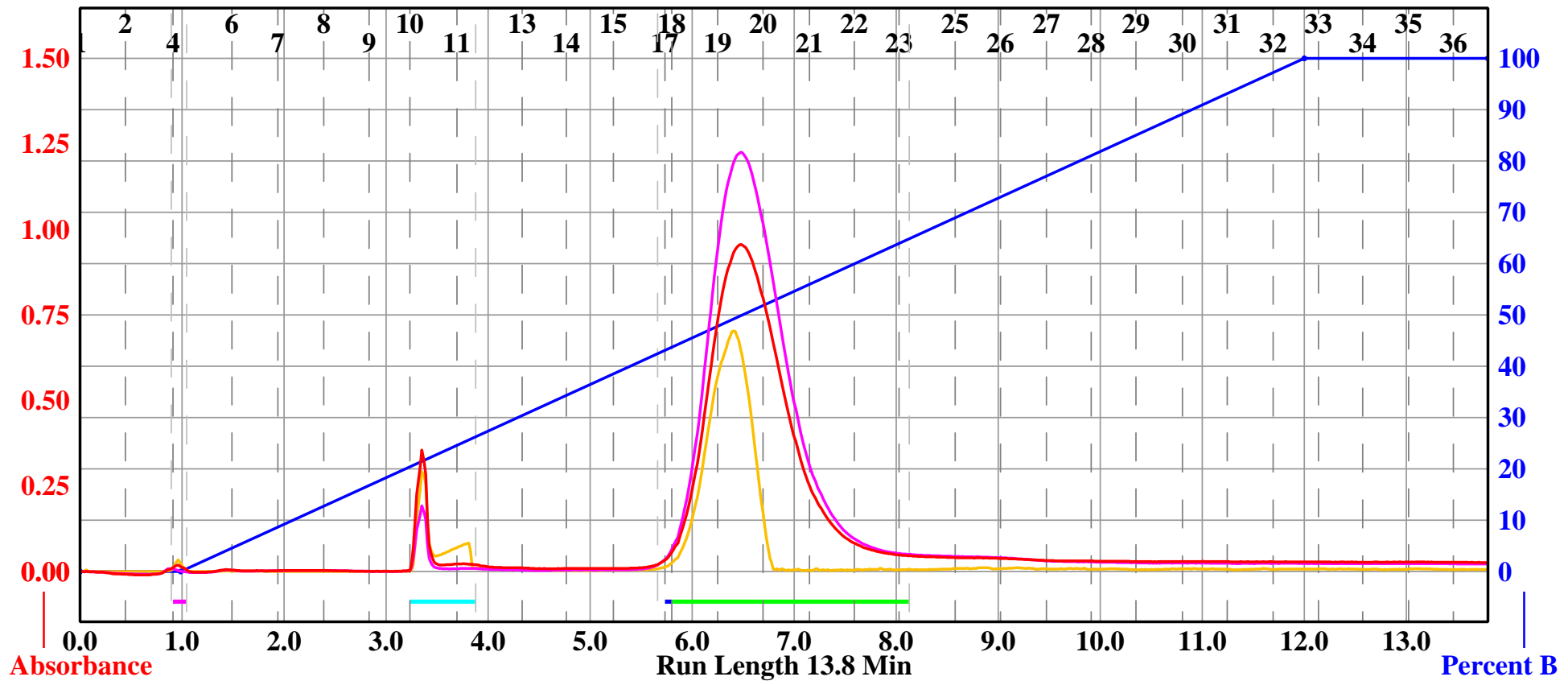

Rack A

Peak \#

Start Tube

End Tube

\begin{tabular}{|c|c|c|c|c|c|}
\hline & (107 & 106 & 105 & (194 & (10? \\
\hline 27 & (8) & (9) & (100) & (1) & (10) \\
\hline$\sqrt{6}$ & (19 & (9) & (3) & (2) & (2) \\
\hline 3 & 8 & 87 & 88 & 89 & (2) \\
\hline 84 & 3 & 82 & 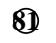 & 810 & (7) \\
\hline 13 & (1) & (28) & (16) & (77) & 18 \\
\hline 12 & (11) & (11) & (9) & 6 & (7) \\
\hline (1) & (2) & (3) & (4) & (6) & ஆ \\
\hline (10) & 59 & 68 & 57 & ஜ & 59 \\
\hline 49 & (D) & (D) & (52) & 53 & 67 \\
\hline 48 & (47) & (46) & (48) & (4) & 43 \\
\hline (37) & B8 & (39) & (41) & (41) & (42) \\
\hline (10) & (3) & B9 & (3) & (12) & (10) \\
\hline 25 & שd & 82 & 68 & (29) & (B) \\
\hline (2) & 23 & 22 & (D) & (21) & (1) \\
\hline (13) & (1) & (15) & (16) & 0 & 18 \\
\hline (12) & (1) & (1) & (2) & (8) & (7) \\
\hline (1) & (2) & (3) & (4) & (5) & (6) \\
\hline
\end{tabular}

$13 \mathrm{~mm} \times 100 \mathrm{~mm}$ Tubes

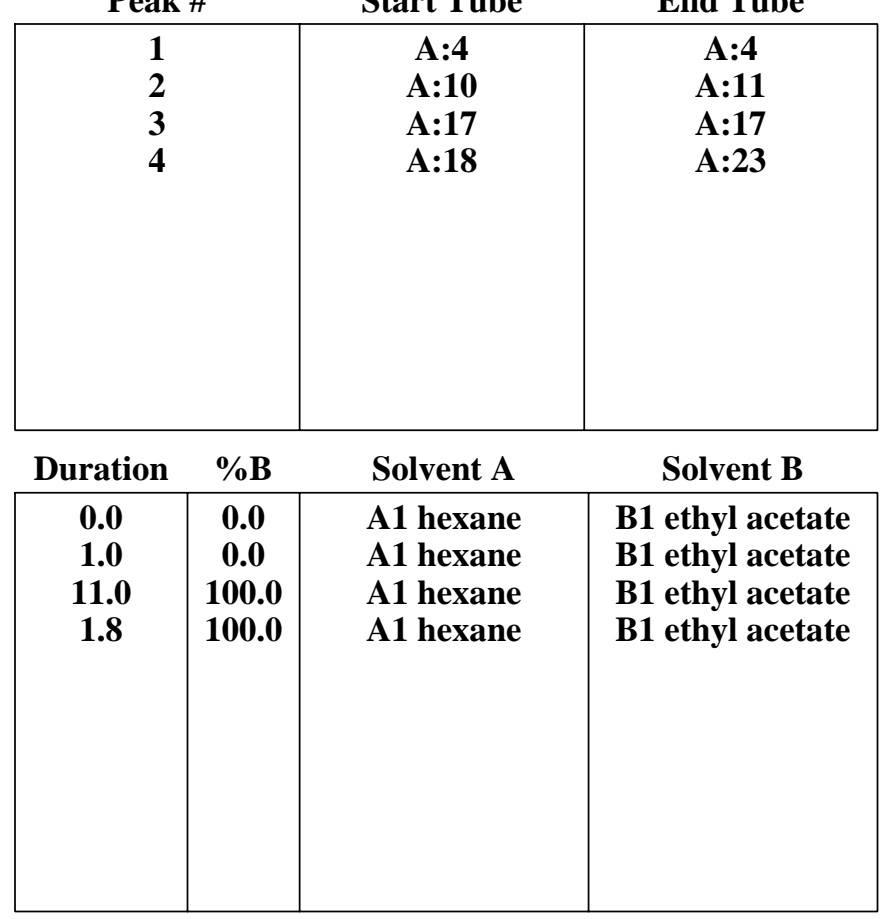


Compound [14]

Sample: ALC-B-238

4x Column: 2

RediSep Column: Silica $4 \mathrm{~g}$

Flow Rate: $18 \mathrm{ml} / \mathrm{min}$

Equilibration Volume: $33.6 \mathrm{ml}$

Initial Waste: $0.0 \mathrm{ml}$

Air Purge: 10.0 min

Solvent: A1 hexane

Solvent: B1 ethyl acetate

\section{Rf 200}

Peak Tube Volume: Max.

Non-Peak Tube Volume: Max.

Loading Type: Solid

Wavelength 1 (red): 254nm

Peak Width: $30 \mathrm{sec}$

Threshold: 0.20 AU

Wavelength 2 (purple): 280nm
Tuesday 22 September 2015 11:01AM

All Wavelength (orange): $200 \mathrm{~nm}-360 \mathrm{~nm}$

Peak Width: 30 sec

Threshold: 0.20 AU

Run Notes:

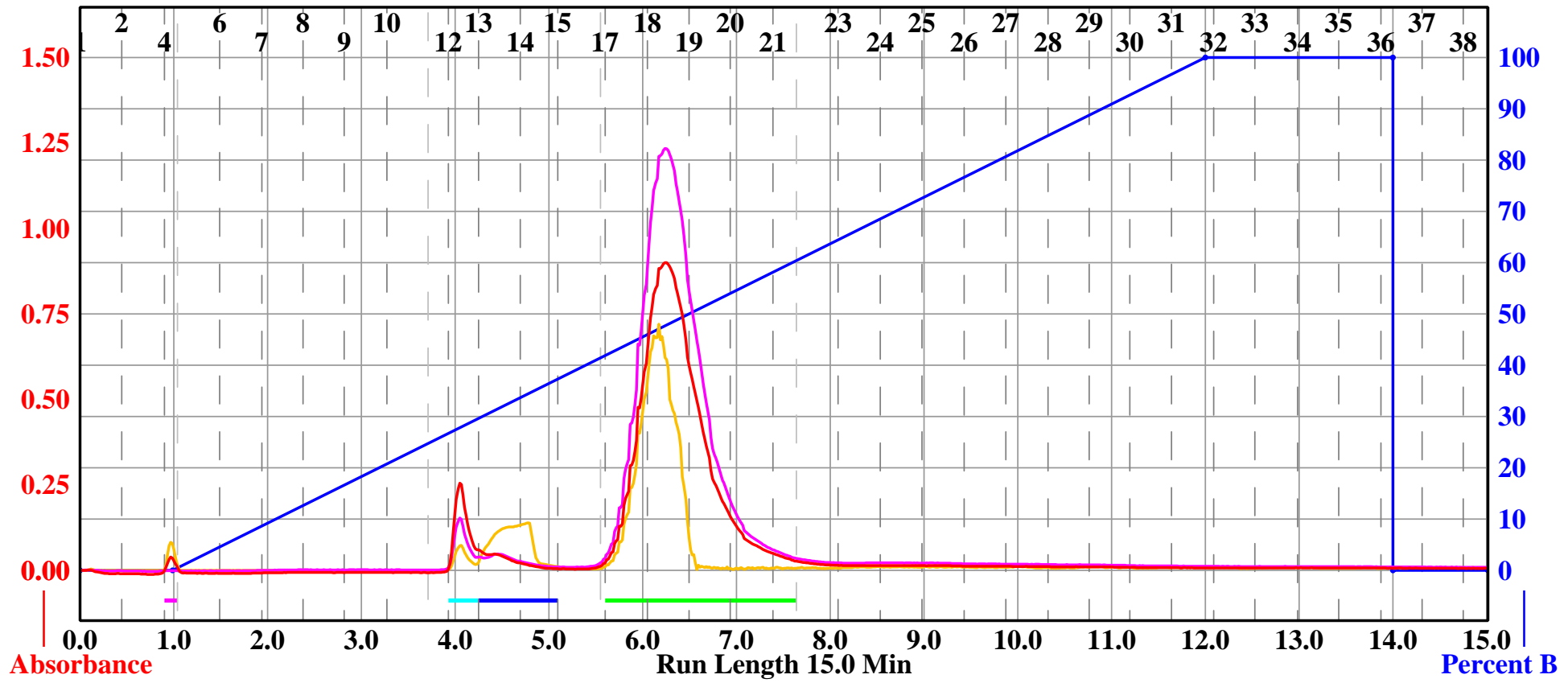

Rack A

Peak \#

Start Tube

End Tube

\begin{tabular}{|c|c|c|c|c|c|}
\hline & (107) & 106 & 105 & (10) & 108 \\
\hline 27 & 18 & (99 & 100 & (10) & $1(102$ \\
\hline 6 & (פ) & (H) & (3) & (2) & (1) \\
\hline 85 & ש6 & 87 & 88 & 89 & (D) \\
\hline 84 & 3 & 82 & (1) & (10) & (19) \\
\hline 13 & 87 & 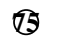 & 『व & $(77$ & 18 \\
\hline$(2$ & (11) & (10) & (99) & 68 & (67) \\
\hline (1) & (2) & (3) & (4) & 68 & ஏ \\
\hline (1) & 59 & 68 & 57 & ๒6 & 5 \\
\hline 49 & (10) & (1) & (2) & 33 & 59 \\
\hline 48 & (47) & (46) & (45) & (4) & (4) \\
\hline (37) & $B 8$ & (99) & (410) & (47) & (42) \\
\hline (1) & (35 & (3) & B3 & (2) & (1D) \\
\hline (25 & 2d & 27 & 88 & 9 & (10) \\
\hline (2) & 23 & 22 & (D) & (2) & (1) \\
\hline C & 0 & (15) & (16) & (1) & 18 \\
\hline (12) & (11) & (1) & (9) & (8) & (7) \\
\hline (1) & (2) & (3) & (4) & (5) & (6) \\
\hline
\end{tabular}

$13 \mathrm{~mm} \times 100 \mathrm{~mm}$ Tubes

\begin{tabular}{|c|c|c|c|}
\hline $\begin{array}{l}1 \\
2 \\
3 \\
4\end{array}$ & & $\begin{array}{c}\text { A:4 } \\
\text { A:12 } \\
\text { A:13 } \\
\text { A:17 }\end{array}$ & $\begin{array}{c}\text { A:4 } \\
\text { A:12 } \\
\text { A:14 } \\
\text { A:21 }\end{array}$ \\
\hline Duration & $\% \mathrm{~B}$ & Solvent A & Solvent B \\
\hline $\begin{array}{c}0.0 \\
1.0 \\
11.0 \\
2.0 \\
0.0 \\
1.0\end{array}$ & \begin{tabular}{|c|}
0.0 \\
0.0 \\
100.0 \\
100.0 \\
0.0 \\
0.0
\end{tabular} & $\begin{array}{l}\text { A1 hexane } \\
\text { A1 hexane } \\
\text { A1 hexane } \\
\text { A1 hexane } \\
\text { A1 hexane } \\
\text { A1 hexane }\end{array}$ & $\begin{array}{l}\text { B1 ethyl acetate } \\
\text { B1 ethyl acetate } \\
\text { B1 ethyl acetate } \\
\text { B1 ethyl acetate } \\
\text { B1 ethyl acetate } \\
\text { B1 ethyl acetate }\end{array}$ \\
\hline
\end{tabular}


4x Column: 2

RediSep Column: Silica 4g

Flow Rate: $18 \mathrm{ml} / \mathrm{min}$

Equilibration Volume: $33.6 \mathrm{ml}$

Initial Waste: $0.0 \mathrm{ml}$

Air Purge: 10.0 min

Solvent: A1 hexane

Solvent: B1 ethyl acetate
Peak Tube Volume: Max.

Non-Peak Tube Volume: Max.

Loading Type: Solid

Wavelength 1 (red): 254nm

Peak Width: 30 sec

Threshold: 0.20 AU

Wavelength 2 (purple): 280nm
All Wavelength (orange): 200nm - 360nm

Peak Width: $30 \mathrm{sec}$

Threshold: 0.20 AU

Run Notes:

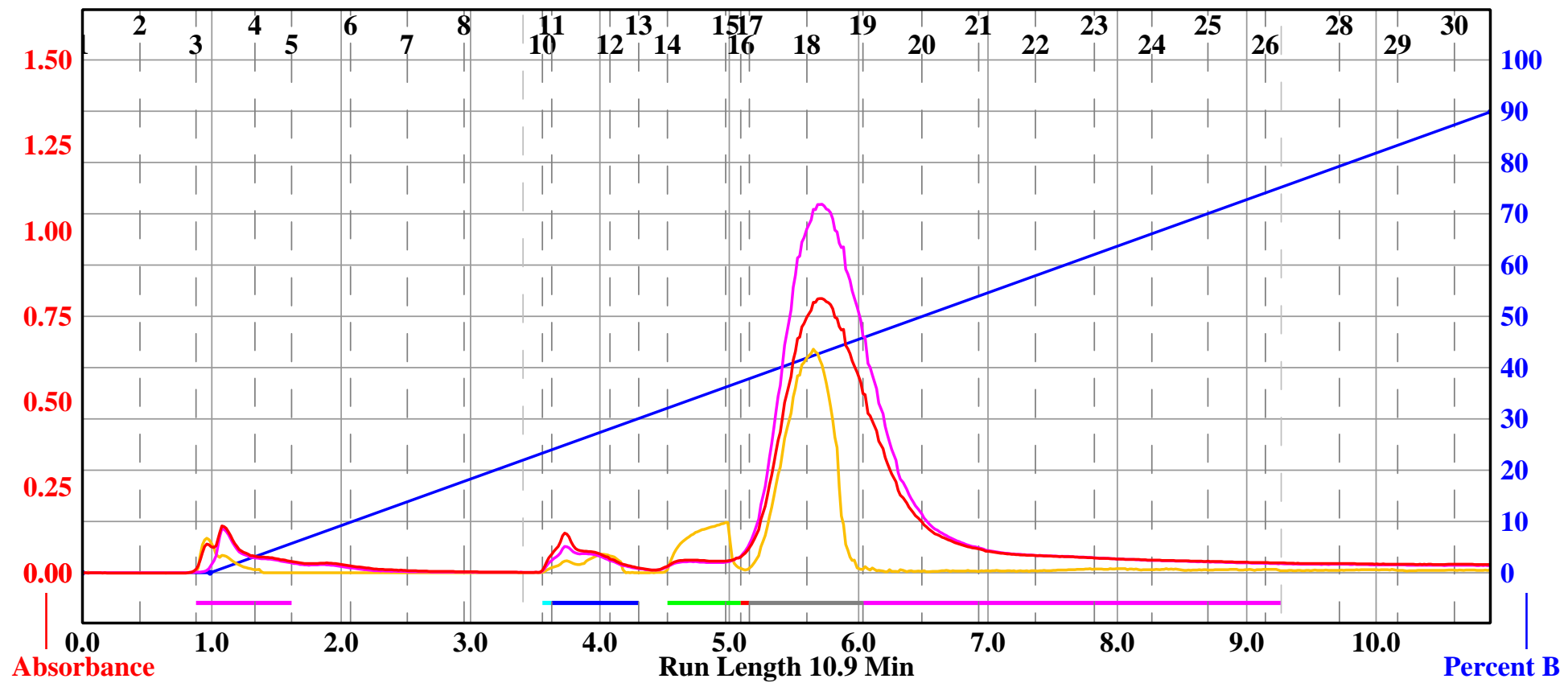

Rack A

Peak \#

Start Tube

End Tube

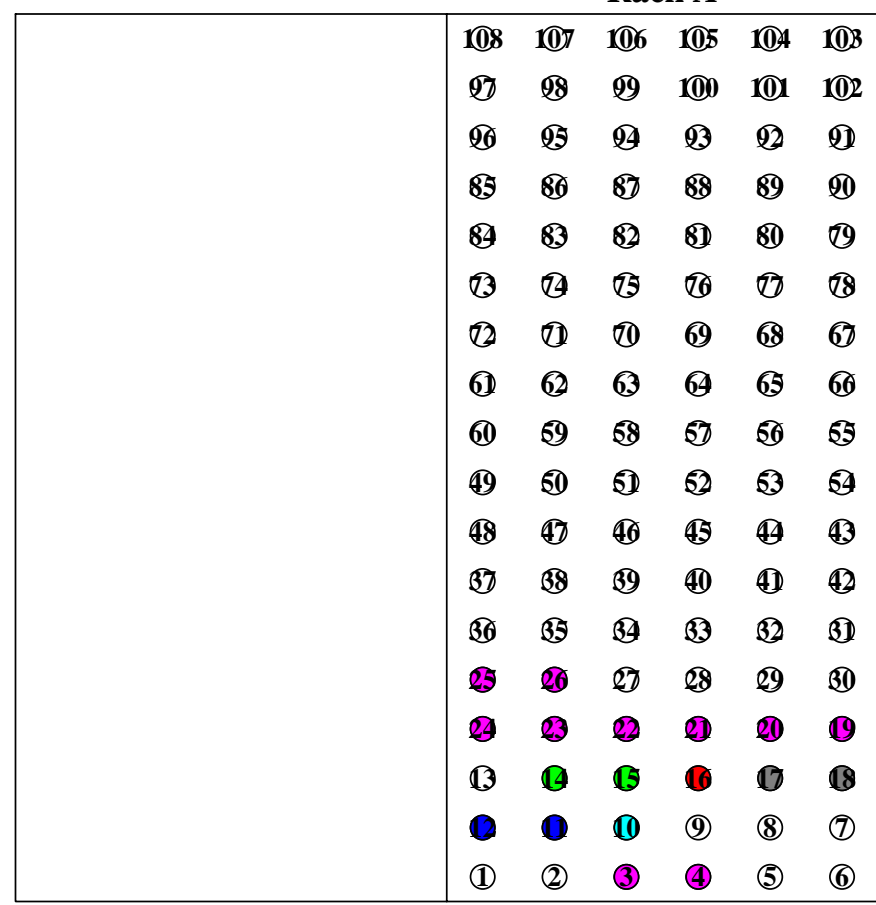

\begin{tabular}{|c|c|c|c|}
\hline $\begin{array}{l}1 \\
2 \\
3 \\
4 \\
5 \\
6 \\
7\end{array}$ & & $\begin{array}{c}\text { A:3 } \\
\text { A:10 } \\
\text { A:11 } \\
\text { A:14 } \\
\text { A:16 } \\
\text { A:17 } \\
\text { A:19 }\end{array}$ & $\begin{array}{c}\text { A:4 } \\
\text { A:10 } \\
\text { A:12 } \\
\text { A:15 } \\
\text { A:16 } \\
\text { A:18 } \\
\text { A:26 }\end{array}$ \\
\hline Duration & $\% B$ & Solvent A & Solvent B \\
\hline $\begin{array}{l}0.0 \\
1.0 \\
9.9\end{array}$ & $\begin{array}{c}0.0 \\
0.0 \\
89.9\end{array}$ & $\begin{array}{l}\text { A1 hexane } \\
\text { A1 hexane } \\
\text { A1 hexane }\end{array}$ & $\begin{array}{l}\text { B1 ethyl acetate } \\
\text { B1 ethyl acetate } \\
\text { B1 ethyl acetate }\end{array}$ \\
\hline
\end{tabular}


Compound [16]

Sample: ALC-C-20

4x Column: 2

RediSep Column: Silica 4g

Flow Rate: $18 \mathrm{ml} / \mathrm{min}$

Equilibration Volume: $33.6 \mathrm{ml}$

Initial Waste: $0.0 \mathrm{ml}$

Air Purge: 10.0 min

Solvent: A1 hexane

Solvent: B1 ethyl acetate

\section{Rf 200}

Thursday 08 October 2015 11:05AM

Peak Tube Volume: Max.

Non-Peak Tube Volume: Max.

Loading Type: Solid

Wavelength 1 (red): 254nm

Peak Width: 30 sec

Threshold: 0.20 AU

Wavelength 2 (purple): 280nm
All Wavelength (orange): 200nm - 360nm

Peak Width: 30 sec

Threshold: 0.20 AU

Run Notes:

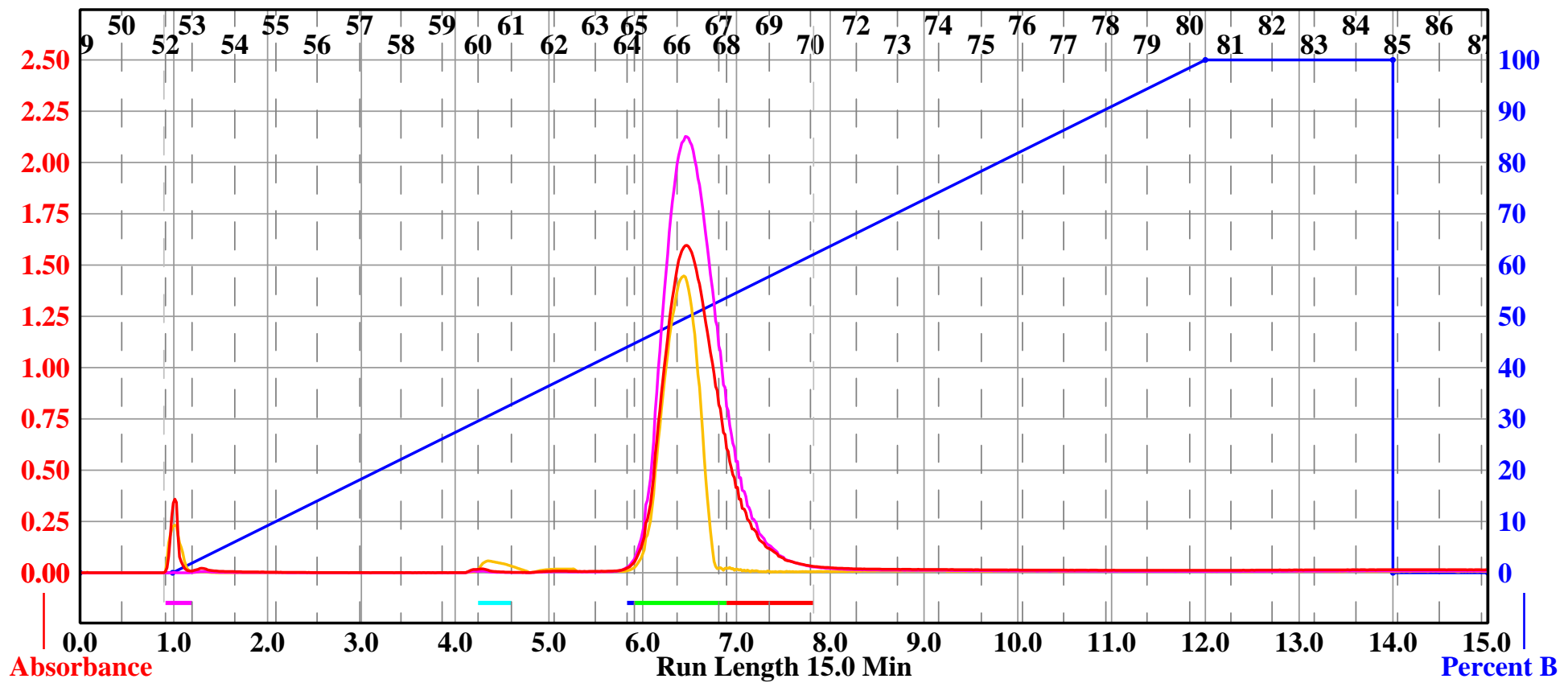

Rack A

Peak \#

Start Tube

End Tube

\begin{tabular}{|c|c|c|c|c|c|}
\hline 18 & (107) & 106 & 105 & (10) & (103 \\
\hline 27 & $\mathscr{O B}$ & (9) & 100 & (1) & $1(102$ \\
\hline Vு & (2) & (צ) & Q & (22) & (1) \\
\hline 35 & 8 & 87 & 88 & 89 & (2) \\
\hline 89 & 83 & 82 & 80 & 80 & (19) \\
\hline 13 & 87 & 18 & (ש) & (17) & 88 \\
\hline 12 & (1) & (1) & (2) & 68 & 67 \\
\hline (1) & (2) & (3) & 0 & 68 & 60 \\
\hline (1) & 59 & 68 & 58 & ஐ & 5 \\
\hline (49) & (D) & (1) & 22 & 53 & 59 \\
\hline 48 & (47) & (46) & 48 & (4) & 43 \\
\hline (37) & B8 & (39) & (41) & (47) & (4) \\
\hline bo & WG & B9 & B3 & (22) & (1D) \\
\hline (25) & שd & 87 & 88 & (2) & (10) \\
\hline (2) & 23 & (2) & (2) & (21) & (1) \\
\hline (1) & (1) & (15) & (16) & (17) & 18 \\
\hline (12) & (11) & (11) & (D) & (8) & (7) \\
\hline (1) & (2) & (3) & (4) & (5) & (6) \\
\hline
\end{tabular}

$13 \mathrm{~mm} \times 100 \mathrm{~mm}$ Tubes

\begin{tabular}{|c|c|c|c|}
\hline $\begin{array}{l}1 \\
2 \\
3 \\
4 \\
5\end{array}$ & & $\begin{array}{l}A: 52 \\
A: 60 \\
A: 64 \\
A: 65 \\
A: 68\end{array}$ & $\begin{array}{l}\text { A:52 } \\
\text { A:60 } \\
\text { A:64 } \\
\text { A:67 } \\
\text { A:70 }\end{array}$ \\
\hline Duration & $\% B$ & Solvent A & Solvent B \\
\hline $\begin{array}{c}0.0 \\
1.0 \\
11.0 \\
2.0 \\
0.0 \\
1.0\end{array}$ & \begin{tabular}{|c|}
0.0 \\
0.0 \\
100.0 \\
100.0 \\
0.0 \\
0.0
\end{tabular} & $\begin{array}{l}\text { A1 hexane } \\
\text { A1 hexane } \\
\text { A1 hexane } \\
\text { A1 hexane } \\
\text { A1 hexane } \\
\text { A1 hexane }\end{array}$ & $\begin{array}{l}\text { B1 ethyl acetate } \\
\text { B1 ethyl acetate } \\
\text { B1 ethyl acetate } \\
\text { B1 ethyl acetate } \\
\text { B1 ethyl acetate } \\
\text { B1 ethyl acetate }\end{array}$ \\
\hline
\end{tabular}


4x Column: 2

RediSep Column: Silica 4g

Flow Rate: $18 \mathrm{ml} / \mathrm{min}$

Equilibration Volume: $33.6 \mathrm{ml}$

Initial Waste: $0.0 \mathrm{ml}$

Air Purge: 10.0 min

Solvent: A1 hexane

Solvent: B1 ethyl acetate
Peak Tube Volume: Max.

Non-Peak Tube Volume: Max.

Loading Type: Solid

Wavelength 1 (red): 254nm

Peak Width: 30 sec

Threshold: 0.20 AU

Wavelength 2 (purple): 280nm
All Wavelength (orange): 200nm - 360nm

Peak Width: $30 \mathrm{sec}$

Threshold: 0.20 AU

Run Notes:

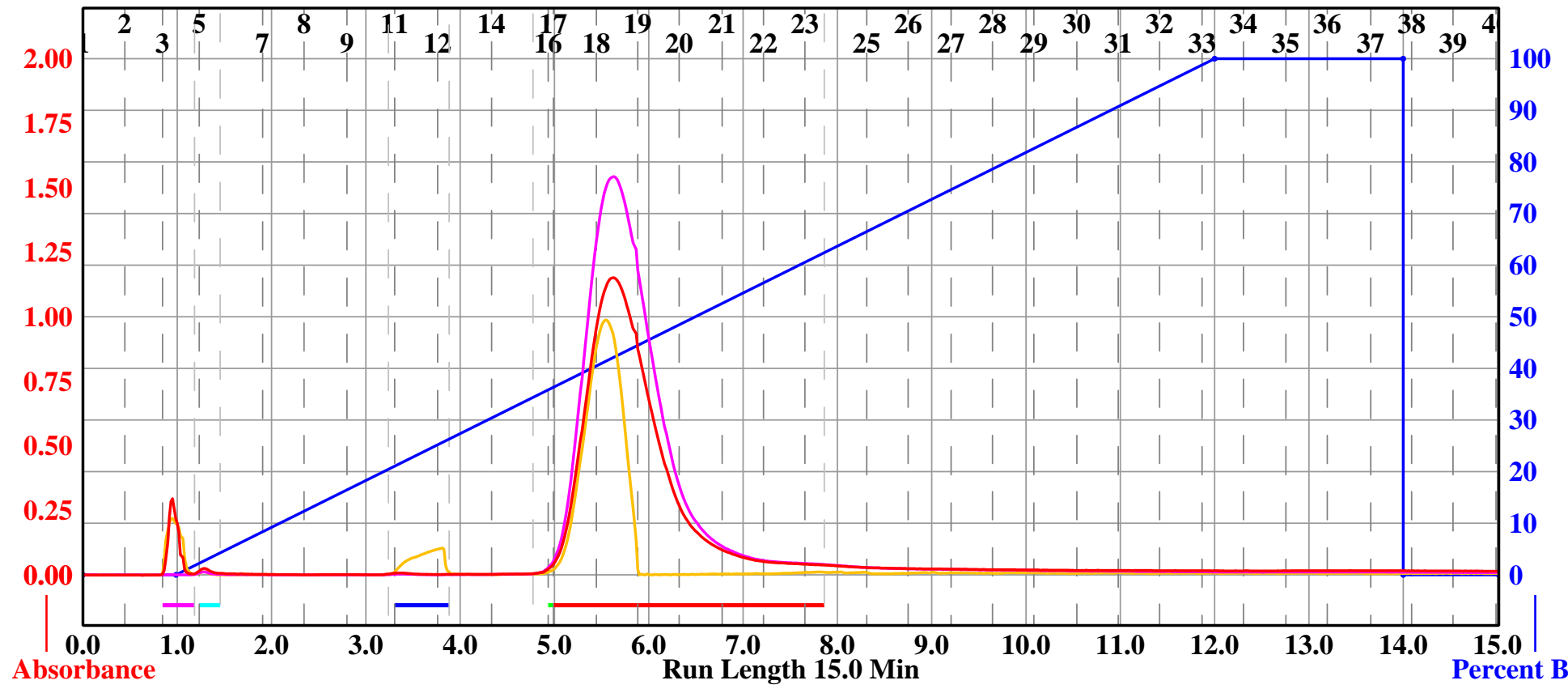

Rack A

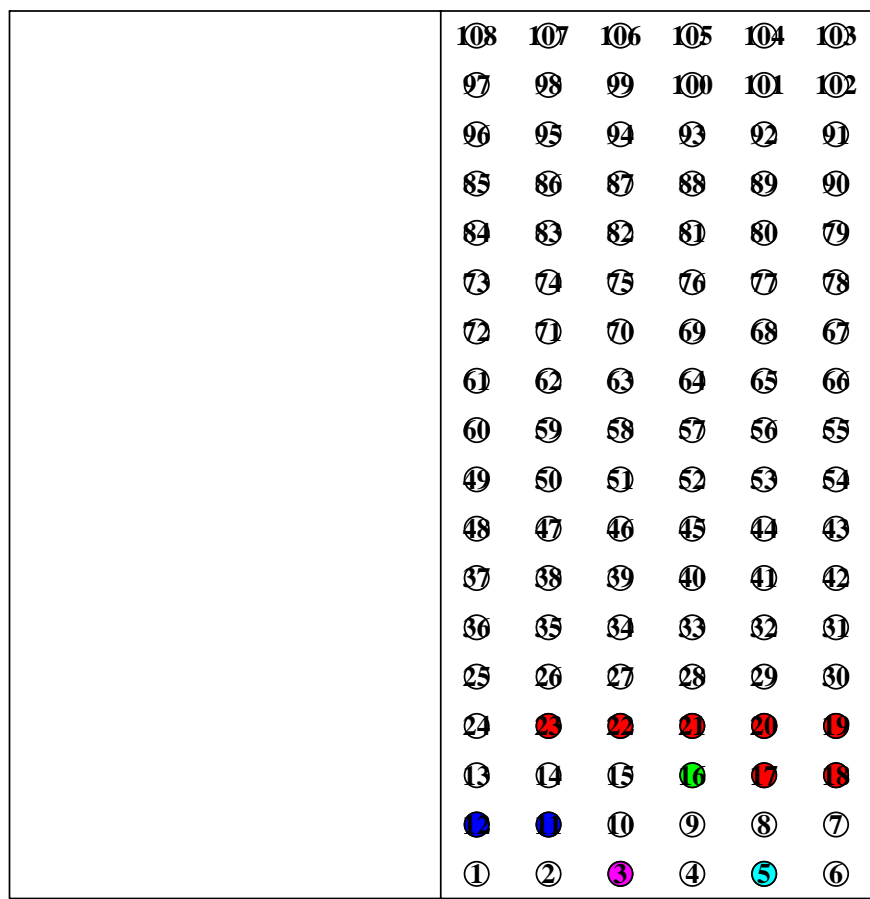

Start Tube $\quad$ End Tube

\begin{tabular}{|c|c|c|c|}
\hline $\begin{array}{l}1 \\
2 \\
3 \\
4 \\
5\end{array}$ & & $\begin{array}{c}\text { A:3 } \\
\text { A:5 } \\
\text { A:11 } \\
\text { A:16 } \\
\text { A:17 }\end{array}$ & $\begin{array}{c}\text { A:3 } \\
\text { A:5 } \\
\text { A:12 } \\
\text { A:16 } \\
\text { A:23 }\end{array}$ \\
\hline Duration & $\% \mathrm{~B}$ & Solvent A & Solvent B \\
\hline $\begin{array}{c}0.0 \\
1.0 \\
11.0 \\
2.0 \\
0.0 \\
1.0\end{array}$ & \begin{tabular}{|c|}
0.0 \\
0.0 \\
100.0 \\
100.0 \\
0.0 \\
0.0
\end{tabular} & $\begin{array}{l}\text { A1 hexane } \\
\text { A1 hexane } \\
\text { A1 hexane } \\
\text { A1 hexane } \\
\text { A1 hexane } \\
\text { A1 hexane }\end{array}$ & $\begin{array}{l}\text { B1 ethyl acetate } \\
\text { B1 ethyl acetate } \\
\text { B1 ethyl acetate } \\
\text { B1 ethyl acetate } \\
\text { B1 ethyl acetate } \\
\text { B1 ethyl acetate }\end{array}$ \\
\hline
\end{tabular}


Compound [18]

Sample: ALC-C-50

4x Column: 3

RediSep Column: Silica 12g

SN: E0415051A8815 Lot: 2418385020W

Flow Rate: $30 \mathrm{ml} / \mathrm{min}$

Equilibration Volume: $100.8 \mathrm{ml}$

Initial Waste: $0.0 \mathrm{ml}$

Air Purge: $10.0 \mathrm{~min}$

Solvent: A1 hexane

Solvent: B1 ethyl acetate

\section{Rf 200}

Peak Tube Volume: Max.

Non-Peak Tube Volume: Max.

Loading Type: Solid

Wavelength 1 (red): 254nm

Peak Width: 1 min

Threshold: 0.20 AU

Wavelength 2 (purple): 280nm
Wednesday 14 October 2015 09:43AM

All Wavelength (orange): 200nm - 360nm

Peak Width: 1 min

Threshold: 0.20 AU

Run Notes:

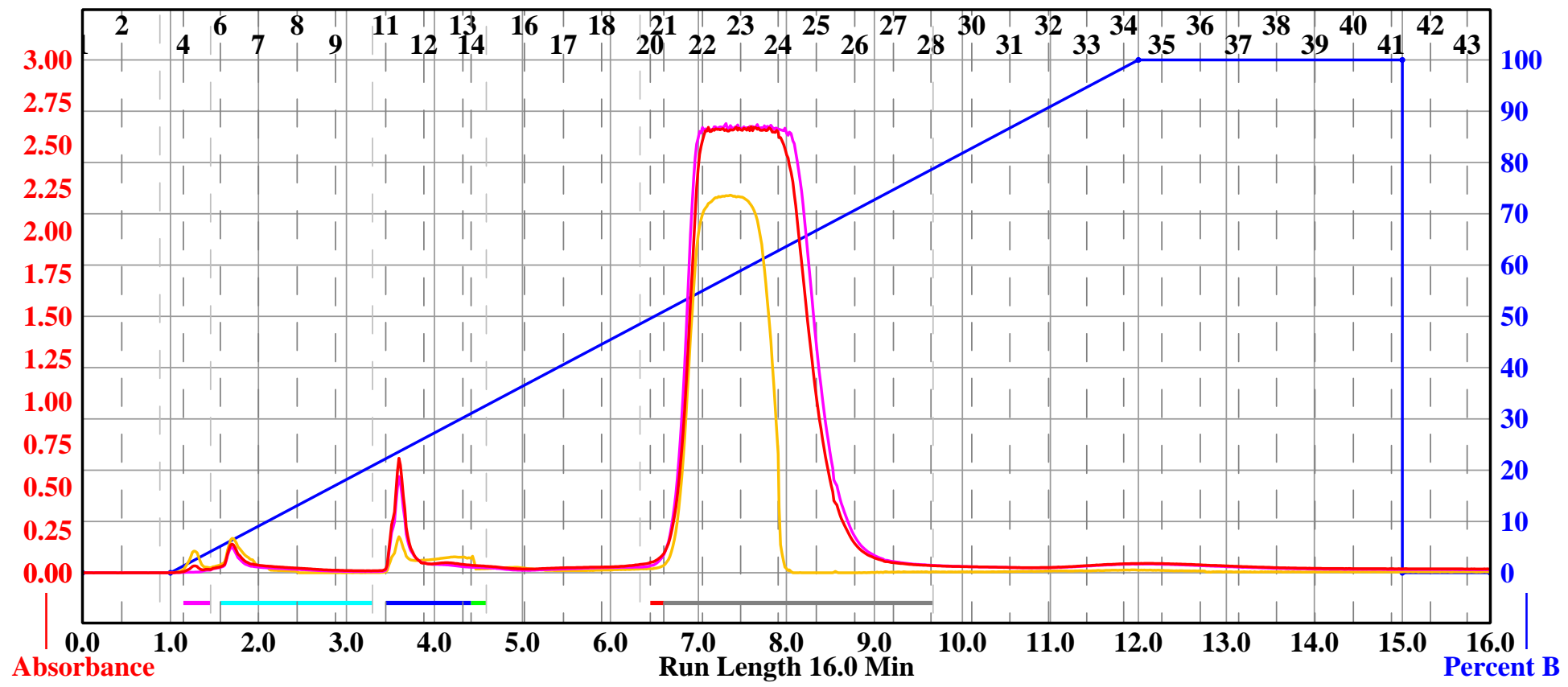

Rack A

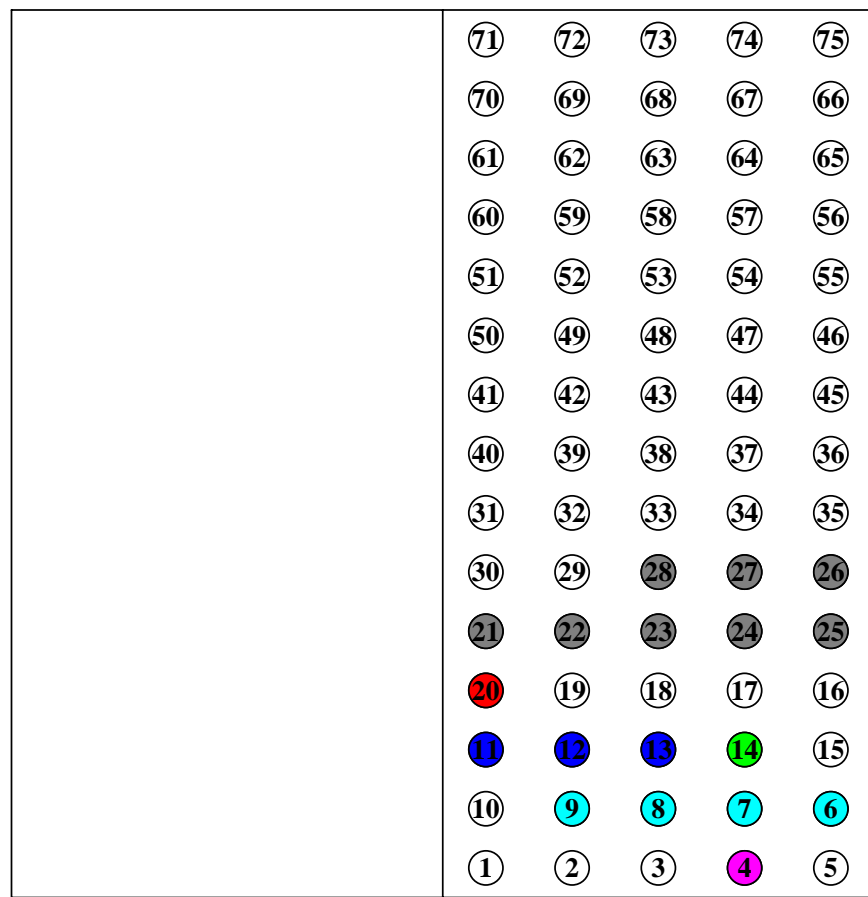

End Tube

\begin{tabular}{c|c} 
Peak \# & Start Tube \\
\hline 1 & A:4
\end{tabular}

A:6

A:11

A:14

A:20

A:21

6

$\sqrt{2}$

(46)

(45)

(36)

(35)

(26)

(25)

(16)

(15)

(6)

(5) 
Rf 200

4x Column: 3

RediSep Column: Silica 4g

Flow Rate: $18 \mathrm{ml} / \mathrm{min}$

Equilibration Volume: $33.6 \mathrm{ml}$

Initial Waste: $0.0 \mathrm{ml}$

Air Purge: $10.0 \mathrm{~min}$

Solvent: A1 hexane

Solvent: B1 ethyl acetate
Peak Tube Volume: Max.

Non-Peak Tube Volume: Max.

Loading Type: Solid

Wavelength 1 (red): 254nm

Peak Width: $30 \mathrm{sec}$

Threshold: 0.20 AU

Wavelength 2 (purple): $280 \mathrm{~nm}$
Wednesday 16 September 2015 10:54AM

All Wavelength (orange): $200 \mathrm{~nm}-360 \mathrm{~nm}$

Peak Width: 30 sec

Threshold: 0.20 AU

Run Notes:

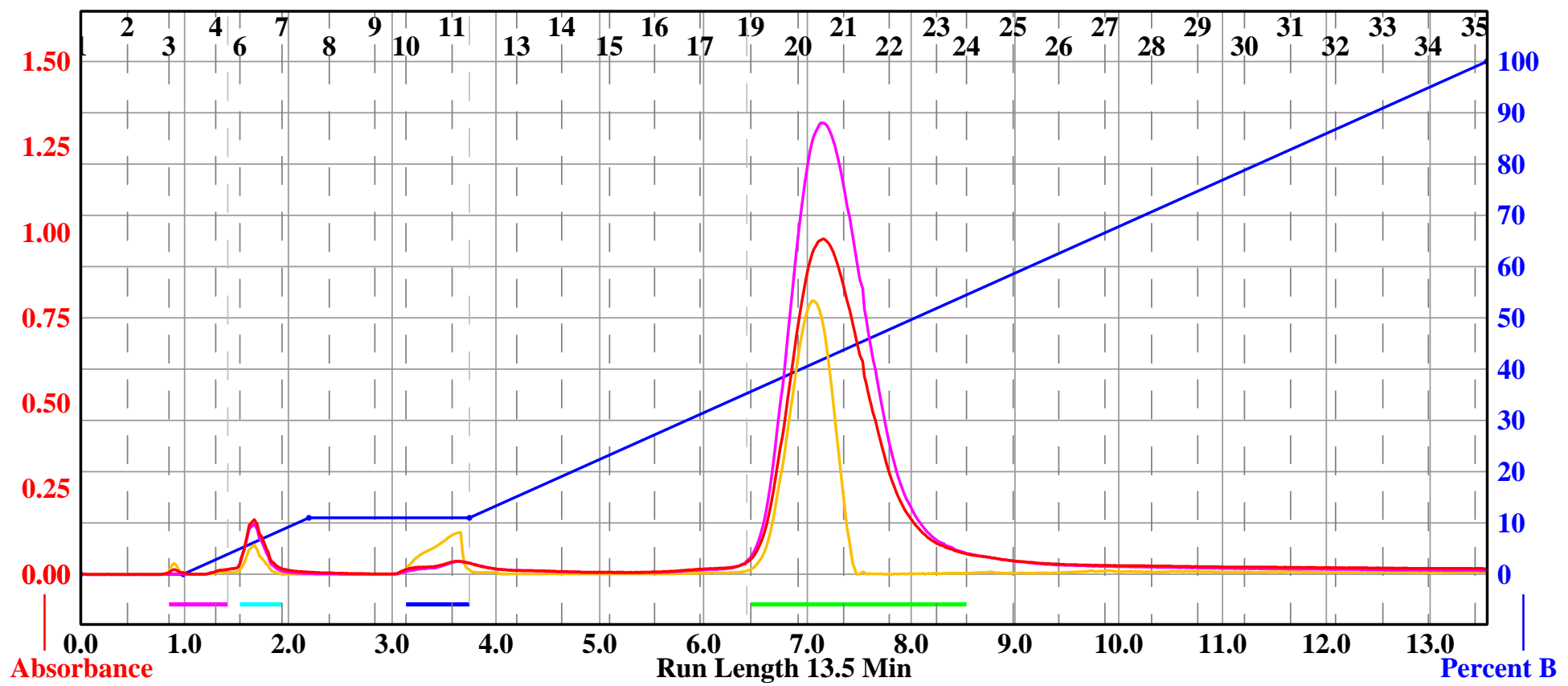

Rack A

\begin{tabular}{|c|c|c|c|c|c|}
\hline & (107) & 106 & 105 & (104 & (103 \\
\hline 27 & Q8 & (9) & (100 & (1) & 102 \\
\hline$\sqrt{6}$ & (2) & (9) & $\mathscr{B}$ & (2) & (D) \\
\hline 15 & ש & 87 & 88 & 89 & (D) \\
\hline 89 & 8 & 82 & 81 & 80 & (29) \\
\hline 3 & 89 & (78) & (8) & (77) & 88 \\
\hline (2) & (11) & (1) & (69) & 68 & 67 \\
\hline (1) & (2) & (3) & (4) & 6 & ன \\
\hline (10 & (9) & 68 & 57 & ஏ) & 58 \\
\hline 9 & 50 & (1) & 52 & 53 & 57 \\
\hline 48 & (47) & (4) & 49 & (4) & 43 \\
\hline (37) & $B$ & (39) & (41) & (41) & (42) \\
\hline (36) & (39) & B9 & B & (22) & (1D) \\
\hline 25 & 26 & 28 & 88 & (29) & (10) \\
\hline 24 & 3 & (2) & (2) & (21) & (1) \\
\hline 13 & (1) & (15) & (16) & (17) & 18 \\
\hline (12) & 0 & 0 & (D) & (8) & (7) \\
\hline (1) & (2) & (3) & (4) & (5) & (6) \\
\hline
\end{tabular}

$13 \mathrm{~mm} \times 100 \mathrm{~mm}$ Tubes

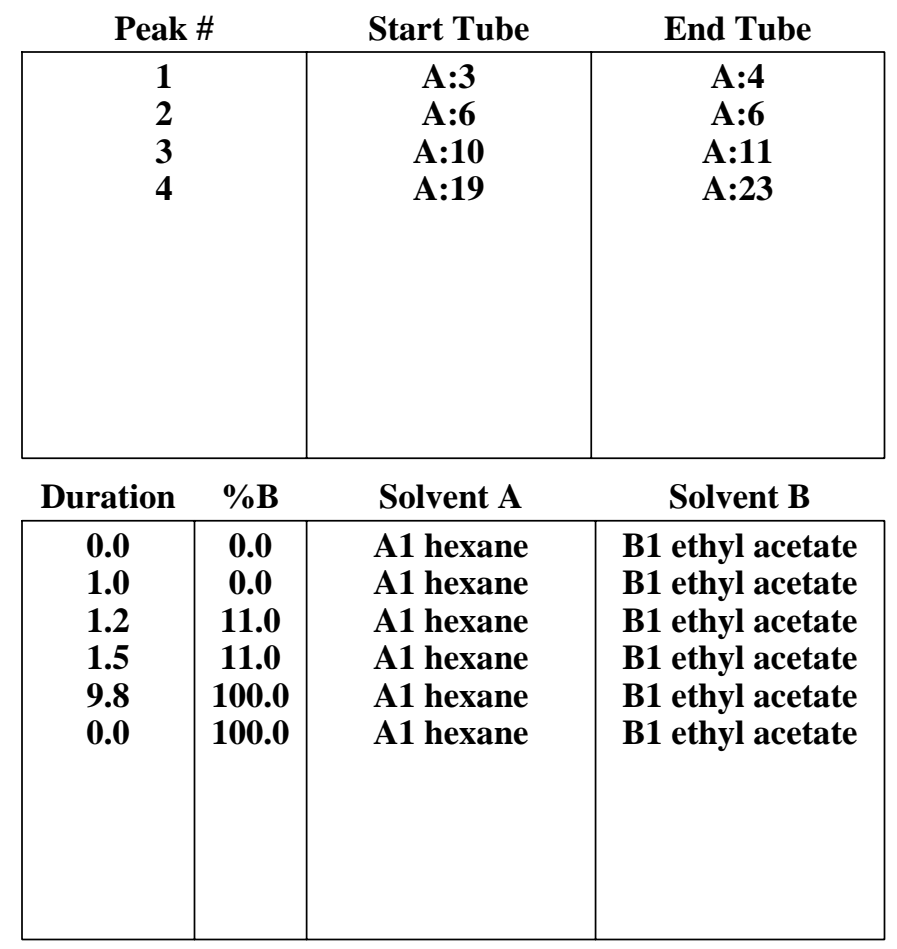


Compound [20]

Sample: ALC-C-24

4x Column: 2

RediSep Column: Silica 4g

Flow Rate: $18 \mathrm{ml} / \mathrm{min}$

Equilibration Volume: $33.6 \mathrm{ml}$

Initial Waste: $0.0 \mathrm{ml}$

Air Purge: $10.0 \mathrm{~min}$

Solvent: A1 hexane

Solvent: B1 ethyl acetate

\section{Rf 200}

Friday 09 October 2015 11:21AM

Peak Tube Volume: Max.

Non-Peak Tube Volume: Max.

Loading Type: Solid

Wavelength 1 (red): 254nm

Peak Width: 30 sec

Threshold: 0.20 AU

Wavelength 2 (purple): 280nm
All Wavelength (orange): 200nm - 360nm

Peak Width: $30 \mathrm{sec}$

Threshold: 0.20 AU

Run Notes:

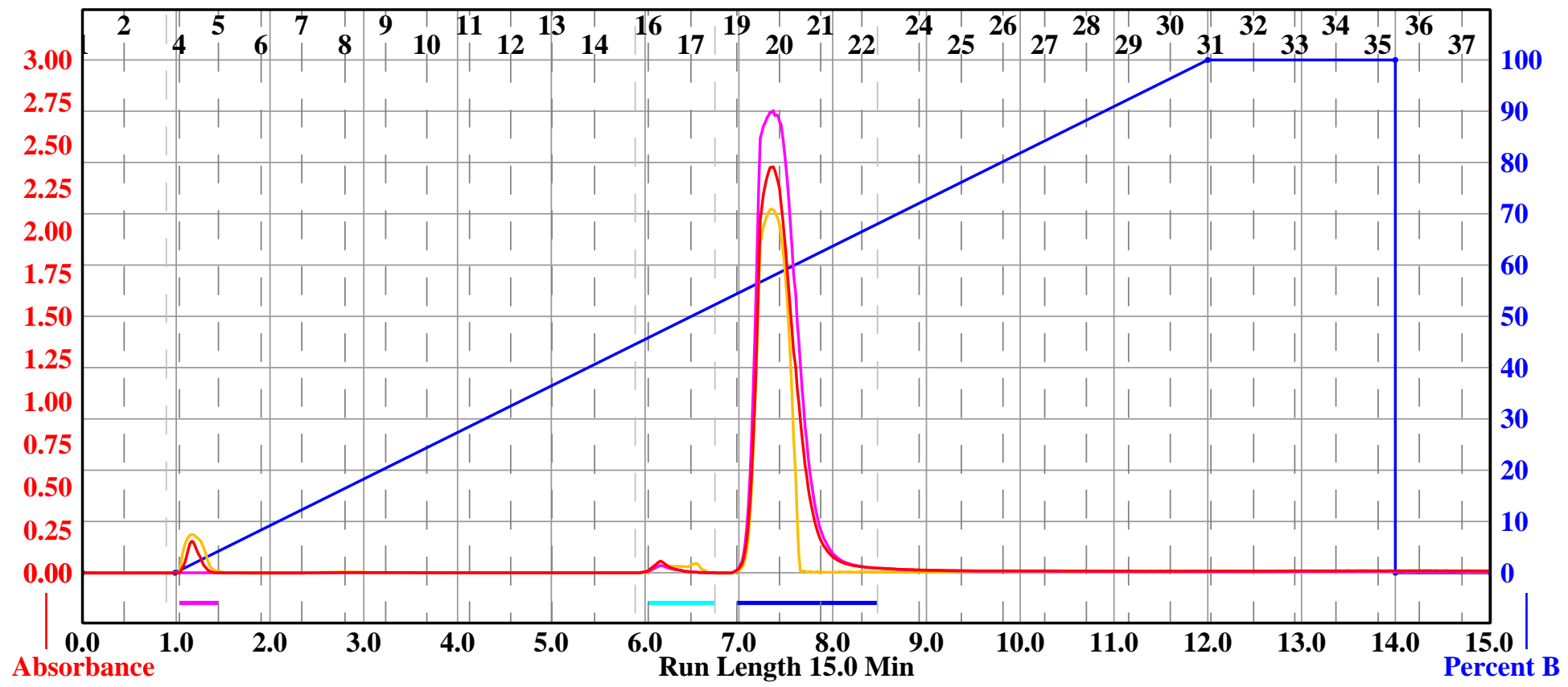

Rack A

\begin{tabular}{|c|c|c|c|c|c|}
\hline & (107 & 106 & 105 & (104 & \\
\hline 27 & 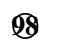 & (9) & (100 & (1)1 & 102 \\
\hline d) & शु & $\mathscr{H}$ & $\mathscr{Q}$ & (2) & (1) \\
\hline 57 & 8 & 87 & 88 & 89 & (D) \\
\hline 84 & 83 & 82 & (1) & 810 & 19 \\
\hline 33 & 18 & (18) & (ש) & (17) & 18 \\
\hline (12) & (1) & (11) & (9) & 6 & (67) \\
\hline (1) & (2) & (3) & (4) & 69 & ช6 \\
\hline (1) & 59 & 88 & 57 & d & 5 \\
\hline (49) & 50 & (D) & 52 & 53 & 59 \\
\hline 48 & (47) & (46) & 45 & (4) & 43 \\
\hline (37) & $B 8$ & (39) & (41) & (41) & (42) \\
\hline (36) & (3) & (3) & B & (32) & (1D) \\
\hline (5) & ๒ & 87 & $\mathfrak{b}$ & (29) & (10) \\
\hline (4) & 23 & e & e & (1) & \\
\hline (13) & (1) & (15) & (16) & (17) & 18 \\
\hline (12) & (1) & (1) & (D) & (8) & (7) \\
\hline (1) & (2) & (3) & (4) & (5) & (6) \\
\hline
\end{tabular}

$13 \mathrm{~mm} \times 100 \mathrm{~mm}$ Tubes

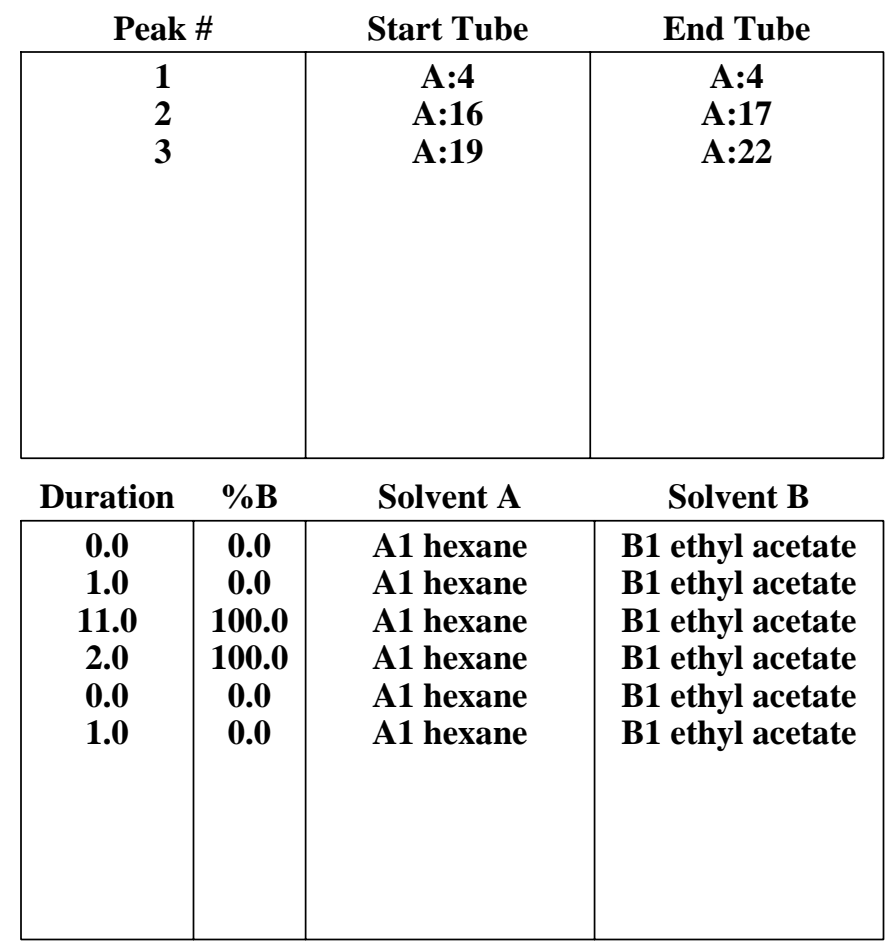


4x Column: 2

RediSep Column: Silica 4g

Flow Rate: $18 \mathrm{ml} / \mathrm{min}$

Equilibration Volume: $33.6 \mathrm{ml}$

Initial Waste: $0.0 \mathrm{ml}$

Air Purge: 10.0 min

Solvent: A1 hexane

Solvent: B1 ethyl acetate
Peak Tube Volume: Max.

Non-Peak Tube Volume: Max.

Loading Type: Solid

Wavelength 1 (red): 254nm

Peak Width: 30 sec

Threshold: 0.20 AU

Wavelength 2 (purple): 280nm
All Wavelength (orange): 200nm - 360nm

Peak Width: $30 \mathrm{sec}$

Threshold: 0.20 AU

Run Notes:

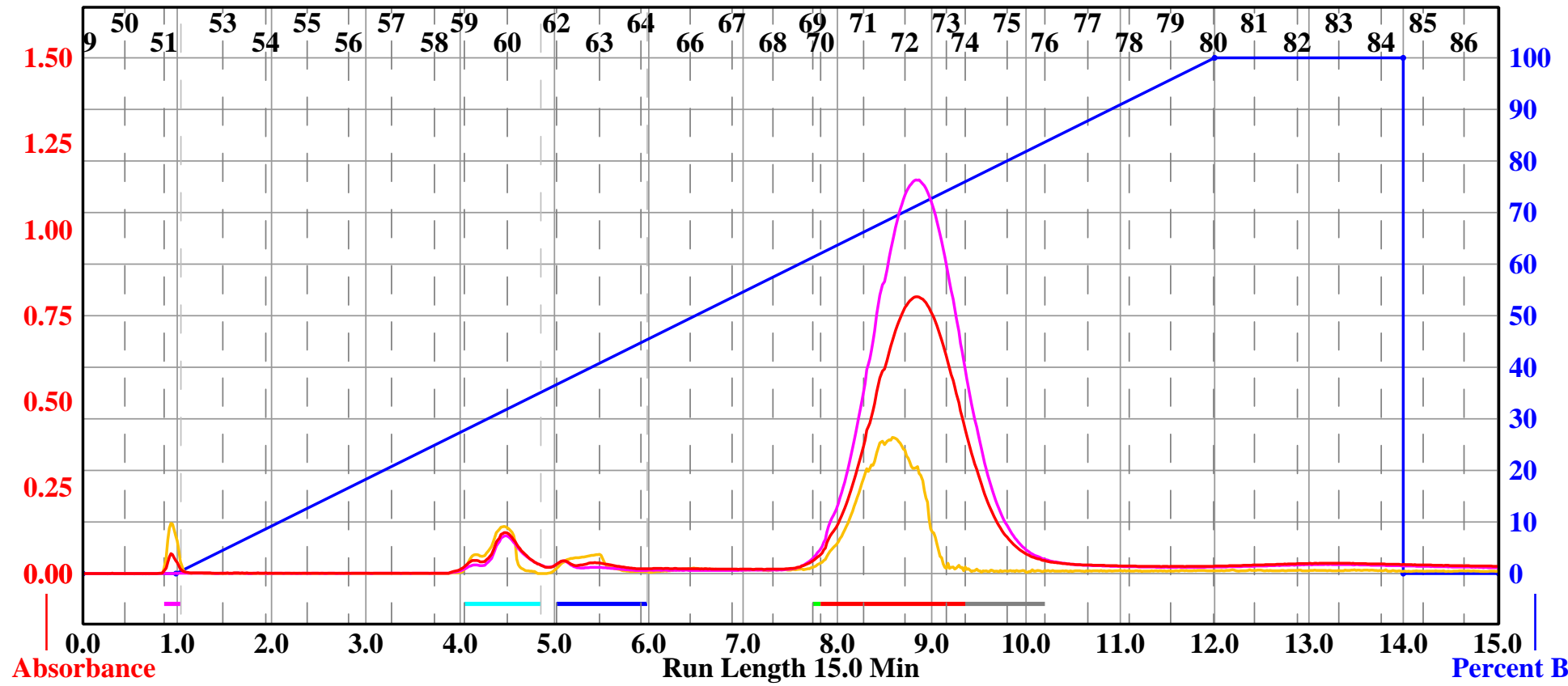

Rack A

Peak \#

Start Tube

End Tube

\begin{tabular}{|c|c|c|c|c|c|}
\hline 08 & (107) & 106 & 105 & (104 & 103 \\
\hline (27) & (B) & (9) & (10) & (1) & $1(102$ \\
\hline dூ & (1) & G & (2) & (2) & (2) \\
\hline 85 & 8 & 87 & 88 & 89 & $\pi$ \\
\hline 84 & 83 & 82 & 80 & (10) & (1) \\
\hline 13 & (1) & 17 & 80 & (87) & 18 \\
\hline 2 & 0 & (1) & (19) & 68 & (17) \\
\hline (1) & 0 & ( & 0 & 69 & 6 \\
\hline (10 & (9) & 88 & 57 & ஏ & 5 \\
\hline (49) & $\mathfrak{b}$ & (1) & 52 & 53 & 59 \\
\hline 48 & (47) & (46) & 4 & (4) & $\mathbb{4}$ \\
\hline (17) & B8 & (39) & (41) & (41) & (42) \\
\hline (B) & (15) & B) & B3 & (2) & (1) \\
\hline 25 & שd & 27 & 68 & (2) & (10) \\
\hline (4) & 3 & (2) & (2) & (21) & (1) \\
\hline (1) & (1) & (1) & (16) & (17) & $\mathbb{1 8}$ \\
\hline (12) & (11) & (10) & (D) & (8) & (7) \\
\hline (1) & (2) & (3) & (4) & (5) & (6) \\
\hline
\end{tabular}

$13 \mathrm{~mm} \times 100 \mathrm{~mm}$ Tubes

\begin{tabular}{|c|c|c|c|}
\hline $\begin{array}{l}\mathbf{1} \\
\mathbf{2} \\
\mathbf{3} \\
\mathbf{4} \\
\mathbf{5} \\
\mathbf{6}\end{array}$ & & $\begin{array}{l}\text { A:51 } \\
\text { A:59 } \\
\text { A:62 } \\
\text { A:69 } \\
\text { A:70 } \\
\text { A:74 }\end{array}$ & $\begin{array}{l}\text { A:51 } \\
\text { A:60 } \\
\text { A:64 } \\
\text { A:69 } \\
\text { A:73 } \\
\text { A:75 }\end{array}$ \\
\hline Duration & $\% \mathrm{~B}$ & Solvent A & Solvent B \\
\hline $\begin{array}{c}0.0 \\
1.0 \\
11.0 \\
2.0 \\
0.0 \\
1.0\end{array}$ & \begin{tabular}{c|}
0.0 \\
0.0 \\
100.0 \\
100.0 \\
0.0 \\
0.0
\end{tabular} & $\begin{array}{l}\text { A1 hexane } \\
\text { A1 hexane } \\
\text { A1 hexane } \\
\text { A1 hexane } \\
\text { A1 hexane } \\
\text { A1 hexane }\end{array}$ & $\begin{array}{l}\text { B1 ethyl acetate } \\
\text { B1 ethyl acetate } \\
\text { B1 ethyl acetate } \\
\text { B1 ethyl acetate } \\
\text { B1 ethyl acetate } \\
\text { B1 ethyl acetate }\end{array}$ \\
\hline
\end{tabular}


4x Column: 3

RediSep Column: Silica 4g

Flow Rate: $18 \mathrm{ml} / \mathrm{min}$

Equilibration Volume: $33.6 \mathrm{ml}$

Initial Waste: $0.0 \mathrm{ml}$

Air Purge: 10.0 min

Solvent: A1 hexane

Solvent: B1 ethyl acetate
Peak Tube Volume: Max.

Non-Peak Tube Volume: Max.

Loading Type: Solid

Wavelength 1 (red): 254nm

Peak Width: 30 sec

Threshold: 0.20 AU

Wavelength 2 (purple): 280nm
All Wavelength (orange): $200 \mathrm{~nm}-360 \mathrm{~nm}$

Peak Width: $30 \mathrm{sec}$

Threshold: 0.20 AU

Run Notes:

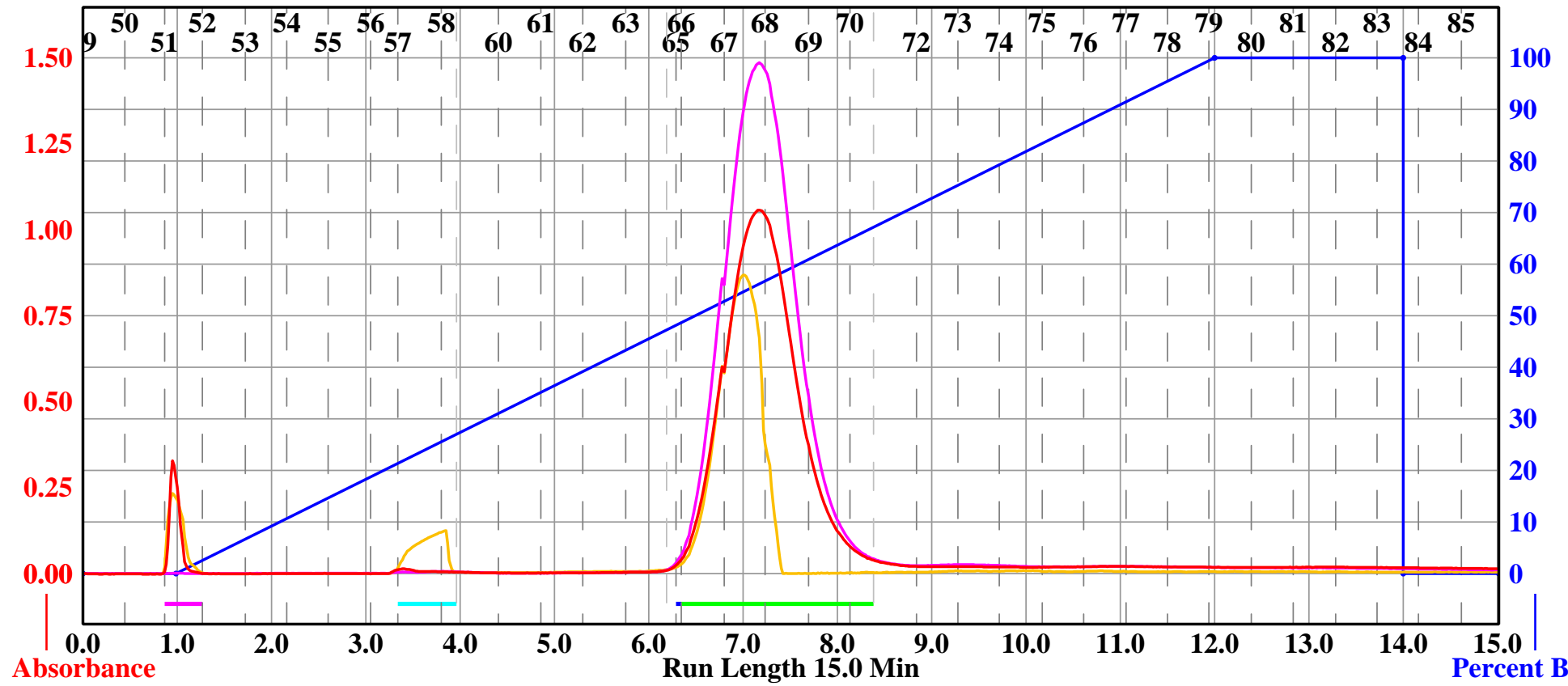

Rack A

Peak \#

Start Tube

End Tube

\begin{tabular}{|c|c|c|c|c|c|}
\hline 8 & (107) & 106 & 105 & (104 & (10? \\
\hline 27 & 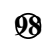 & (9) & (100 & (1) & (10) \\
\hline D & (2) & $\mathscr{H}$ & Q3 & (2) & (1) \\
\hline 85 & 8 & 87 & 88 & 89 & (2) \\
\hline 34 & 83 & 82 & 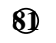 & 80 & (79) \\
\hline 83 & (8) & (15) & (20) & (17) & 88 \\
\hline 22 & (11) & (11) & (6) & 68 & (67) \\
\hline (1) & (2) & (3) & 8 & (1) & (6) \\
\hline (11) & 59 & 88 & (57) & ஐb & 65 \\
\hline 49 & (10) & (1) & (52) & 53 & 59 \\
\hline 48 & (47) & (46) & 49 & 49 & 43 \\
\hline (37) & B8 & B9 & (40) & (47) & (42) \\
\hline bV & B5 & B & B3 & (32) & (1) \\
\hline (25) & (2) & 82 & 88 & (2) & (10) \\
\hline (4) & 23 & 22 & (2) & (20) & (19) \\
\hline (13) & (1) & (15) & (16) & (17) & 18 \\
\hline (12) & (1) & (1) & (D) & (8) & (7) \\
\hline (1) & (2) & (3) & (4) & (5) & (6) \\
\hline
\end{tabular}

$13 \mathrm{~mm} \times 100 \mathrm{~mm}$ Tubes

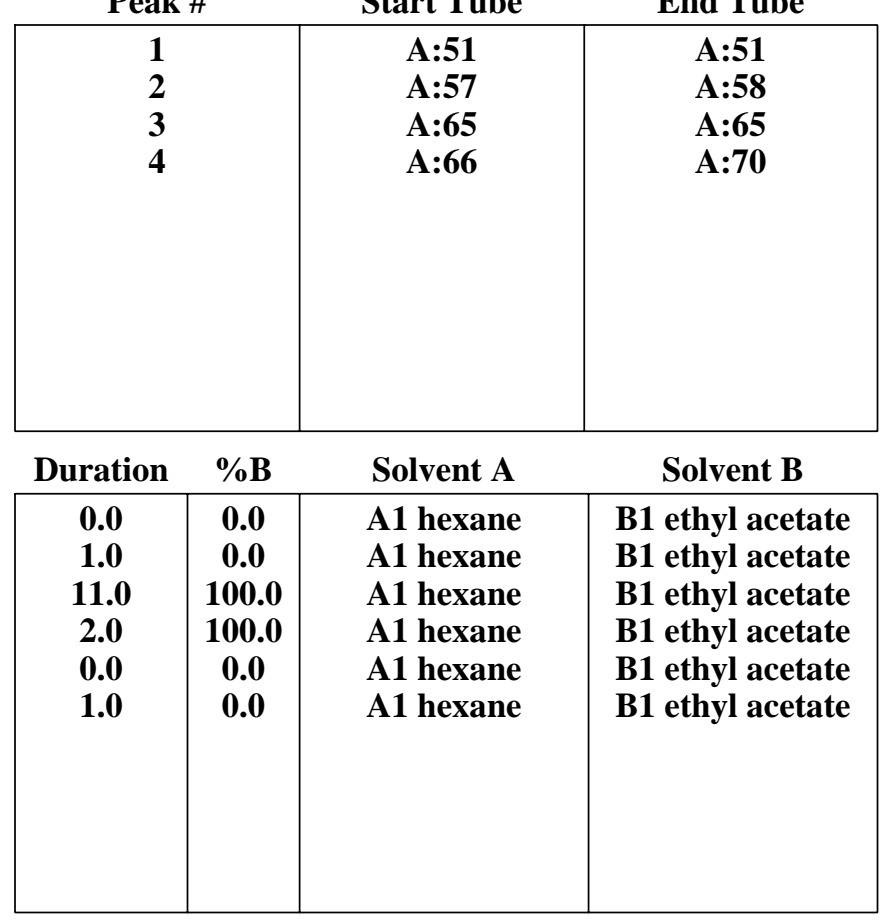


4x Column: 2

RediSep Column: Silica 4g

Flow Rate: $18 \mathrm{ml} / \mathrm{min}$

Equilibration Volume: $33.6 \mathrm{ml}$

Initial Waste: $0.0 \mathrm{ml}$

Air Purge: 10.0 min

Solvent: A1 hexane

Solvent: B1 ethyl acetate
Peak Tube Volume: Max.

Non-Peak Tube Volume: Max.

Loading Type: Solid

Wavelength 1 (red): 254nm

Peak Width: 30 sec

Threshold: 0.20 AU

Wavelength 2 (purple): 280nm
All Wavelength (orange): 200nm - 360nm

Peak Width: 30 sec

Threshold: 0.20 AU

Run Notes:

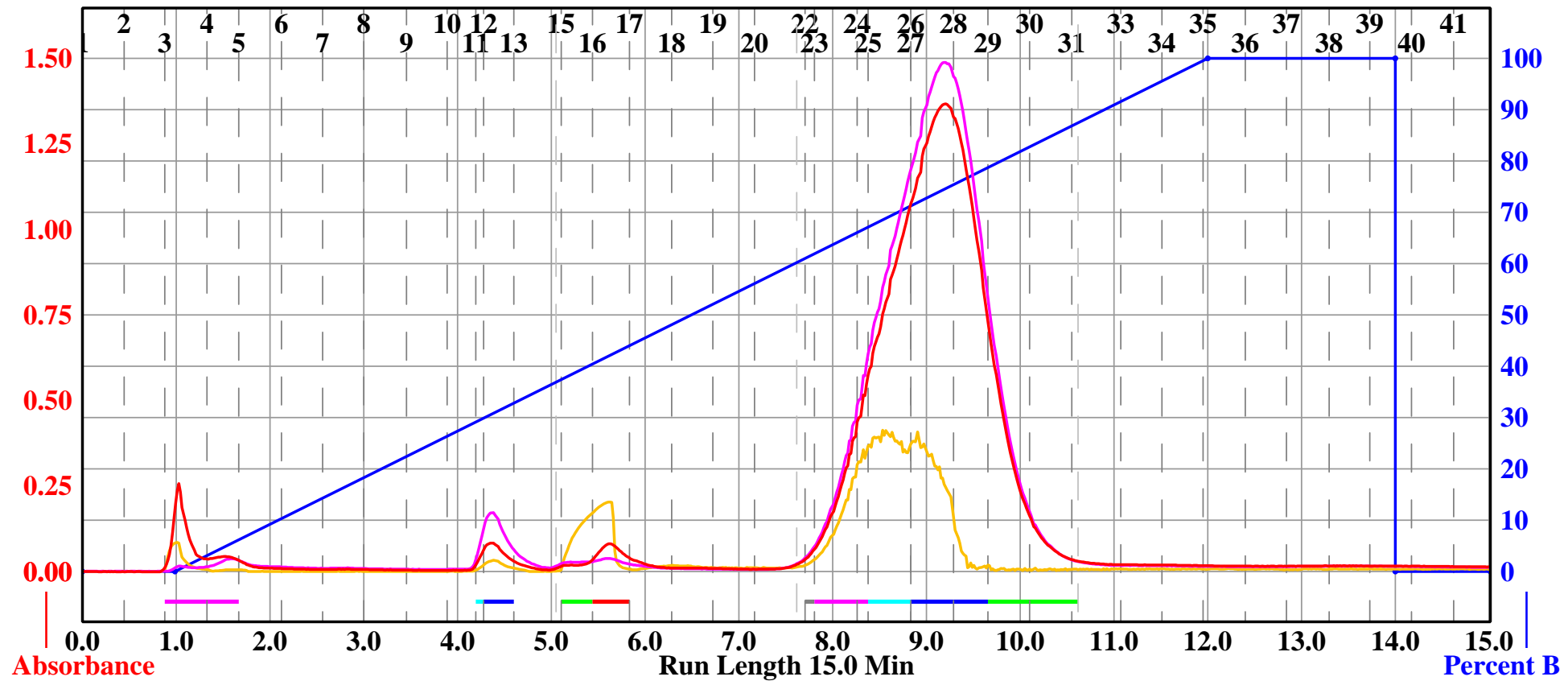

Rack A

Peak \#

Start Tube

End Tube

\begin{tabular}{|c|c|c|c|c|c|}
\hline & (107) & 106 & 105 & (104 & 1 (103 \\
\hline 27 & (8) & (9) & (10) & (1) & (10) \\
\hline df & Ð & $\theta$ & (3) & (2) & (1) \\
\hline 87 & 8 & 87 & 88 & 89 & ஜ2) \\
\hline 89 & 83 & 82 & 80 & 80 & (29) \\
\hline 10 & (27) & 0 & (20) & $(77$ & 88 \\
\hline (2) & (7) & (12) & (9) & 68 & 67 \\
\hline (1) & (2) & 63 & (4) & 6 & ஏ \\
\hline (10) & 59 & 58 & 57 & ஏ0 & 5 \\
\hline (49) & (90) & (D) & (52) & 63 & 59 \\
\hline 48 & (47) & (46) & (45) & (4) & 43 \\
\hline (37) & B8 & (39) & (41) & (17) & (42) \\
\hline Bd & BS & B9 & B3 & (32) & (D) \\
\hline 2 & שd & 0 & 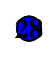 & (29) & (31) \\
\hline (2) & 23 & (2) & (2) & (2) & (19) \\
\hline (1) & (1) & (1) & 0 & (17) & 18 \\
\hline 0 & (1) & (1) & (9) & (8) & (7) \\
\hline (1) & (2) & (3) & (4) & (5) & (6) \\
\hline
\end{tabular}

$13 \mathrm{~mm} \times 100 \mathrm{~mm}$ Tubes

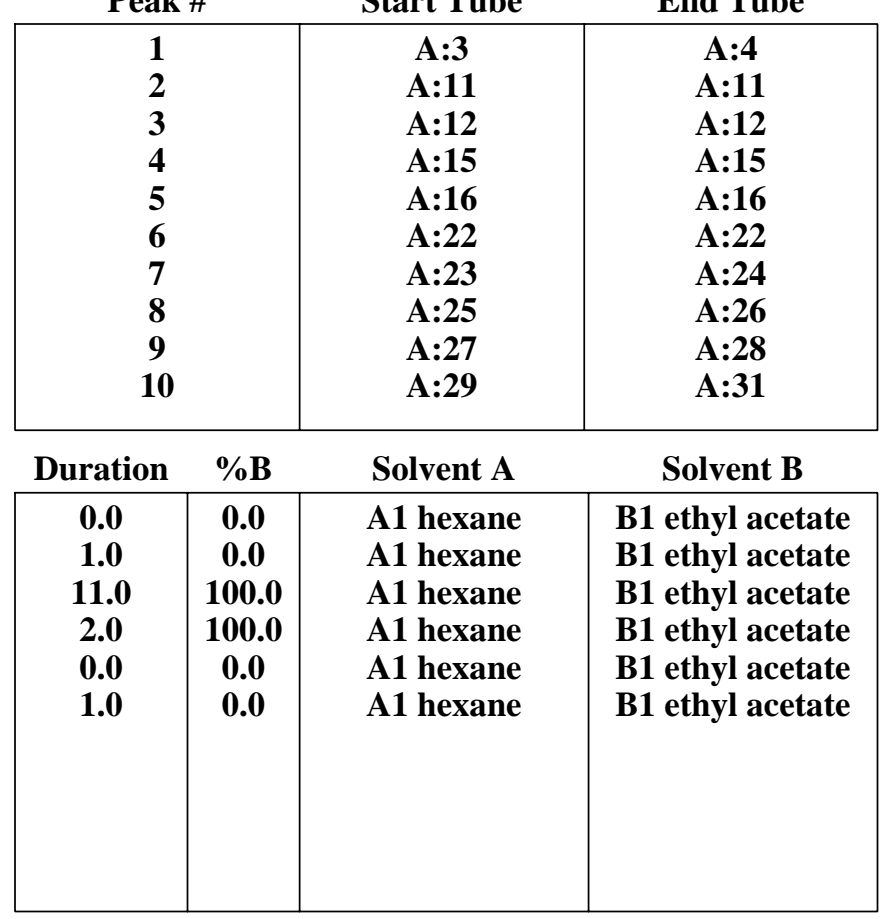


Compound [24]

Sample: ALC-C-28

4x Column: 2

RediSep Column: Silica 4g

Flow Rate: $18 \mathrm{ml} / \mathrm{min}$

Equilibration Volume: $33.6 \mathrm{ml}$

Initial Waste: $0.0 \mathrm{ml}$

Air Purge: $10.0 \mathrm{~min}$

Solvent: A1 hexane

Solvent: B1 ethyl acetate

\section{Rf 200}

Peak Tube Volume: Max.

Non-Peak Tube Volume: Max.

Loading Type: Solid

Wavelength 1 (red): 254nm

Peak Width: $30 \mathrm{sec}$

Threshold: 0.20 AU

Wavelength 2 (purple): $280 \mathrm{~nm}$
Wednesday 14 October 2015 02:46PM

All Wavelength (orange): $200 \mathrm{~nm}-360 \mathrm{~nm}$

Peak Width: 30 sec

Threshold: 0.20 AU

Run Notes:

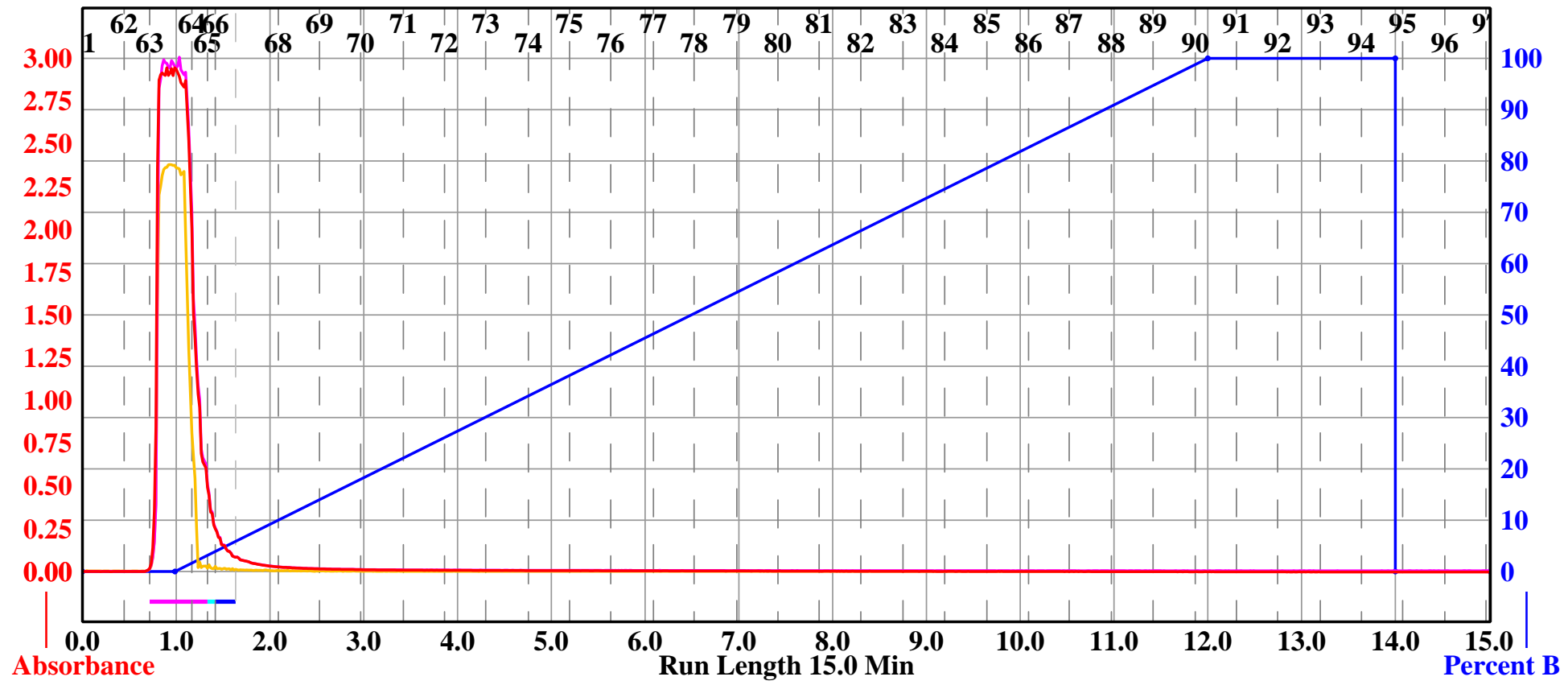

Rack A

\begin{tabular}{|c|c|c|c|c|c|}
\hline & (107) & 106 & (105 & (104 & 1(103 \\
\hline 27 & 18 & (19) & (100 & (1) & 102 \\
\hline d & (19 & (29 & 13 & (22) & (D) \\
\hline 35 & 8 & 87 & 88 & 89 & (D) \\
\hline 34 & 8 & 82 & (1) & 80 & (19) \\
\hline 83 & 89 & (18) & (8) & (77) & 18 \\
\hline 12 & (1) & (1) & (6) & 68 & (67) \\
\hline (1) & (2) & (3) & (8) & (6) & 0 \\
\hline (1) & 59 & $\mathfrak{8 8}$ & 57 & छ) & 5 \\
\hline 49 & $\mathfrak{b}$ & (D) & 52 & 5 & 59 \\
\hline 48 & (47) & (46) & 49 & (4) & 43 \\
\hline 37 & $\mathfrak{B P}$ & (99) & (41) & (41) & (42) \\
\hline (36) & BS & (3) & B3 & (2) & (1D) \\
\hline 25 & (2) & 27 & 28 & (29) & (10) \\
\hline 24 & 3 & 22 & (2) & (21) & (1) \\
\hline (13) & (1) & (15) & (16) & (17) & 18 \\
\hline (12) & (1) & (1) & (D) & (8) & (7) \\
\hline (1) & (2) & (3) & (4) & (5) & (6) \\
\hline
\end{tabular}

$13 \mathrm{~mm} \times 100 \mathrm{~mm}$ Tubes

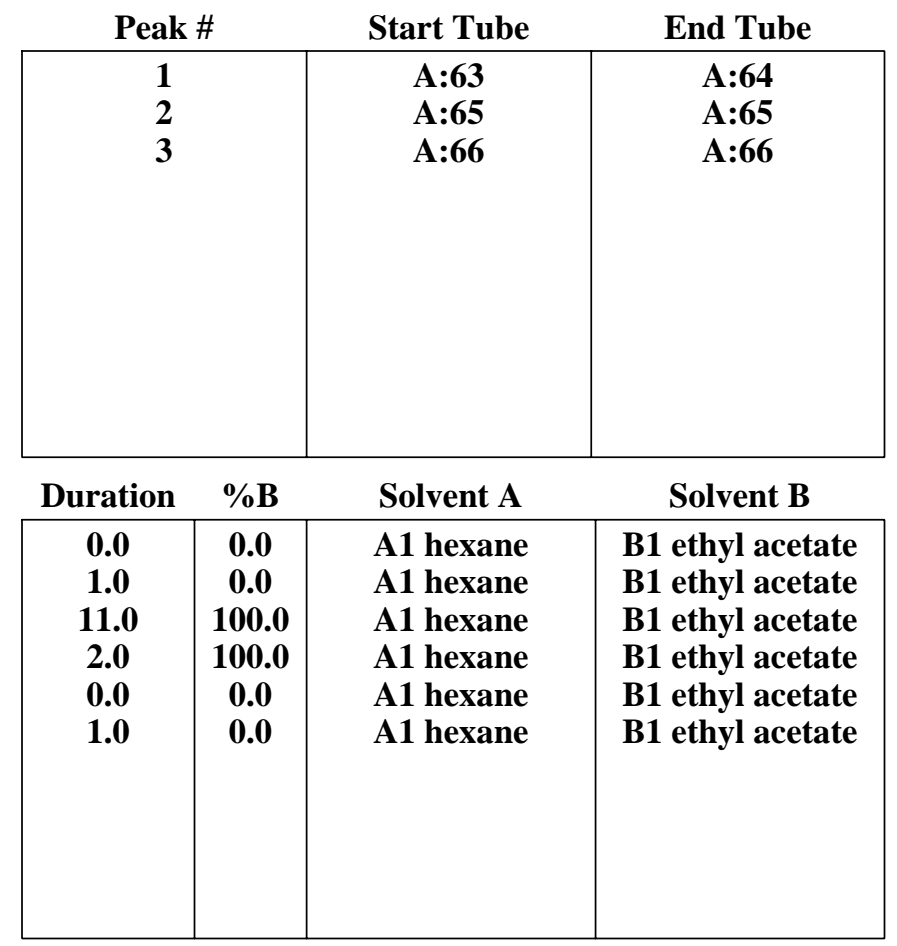


Compound [25]

Sample: ALC-B-258

4x Column: 2

RediSep Column: Silica 4g

Flow Rate: $18 \mathrm{ml} / \mathrm{min}$

Equilibration Volume: $33.6 \mathrm{ml}$

Initial Waste: $0.0 \mathrm{ml}$

Air Purge: 10.0 min

Solvent: A1 hexane

Solvent: B1 ethyl acetate

\section{Rf 200}

Peak Tube Volume: Max.

Non-Peak Tube Volume: Max.

Loading Type: Solid

Wavelength 1 (red): 254nm

Peak Width: 30 sec

Threshold: 0.20 AU

Wavelength 2 (purple): 280nm
Thursday 24 September 2015 10:04AM

All Wavelength (orange): 200nm - 360nm

Peak Width: $30 \mathrm{sec}$

Threshold: 0.20 AU

Run Notes:

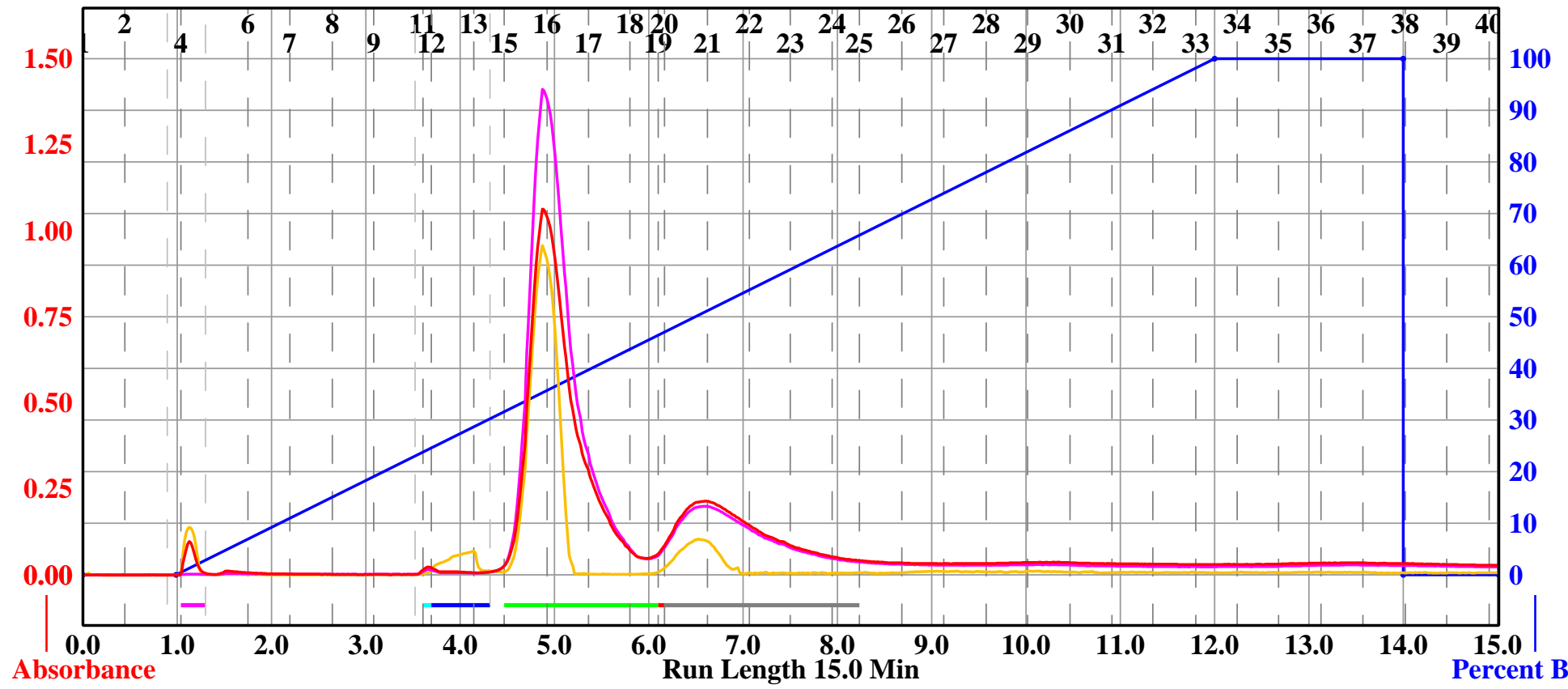

Rack A

Peak \#

Start Tube

End Tube

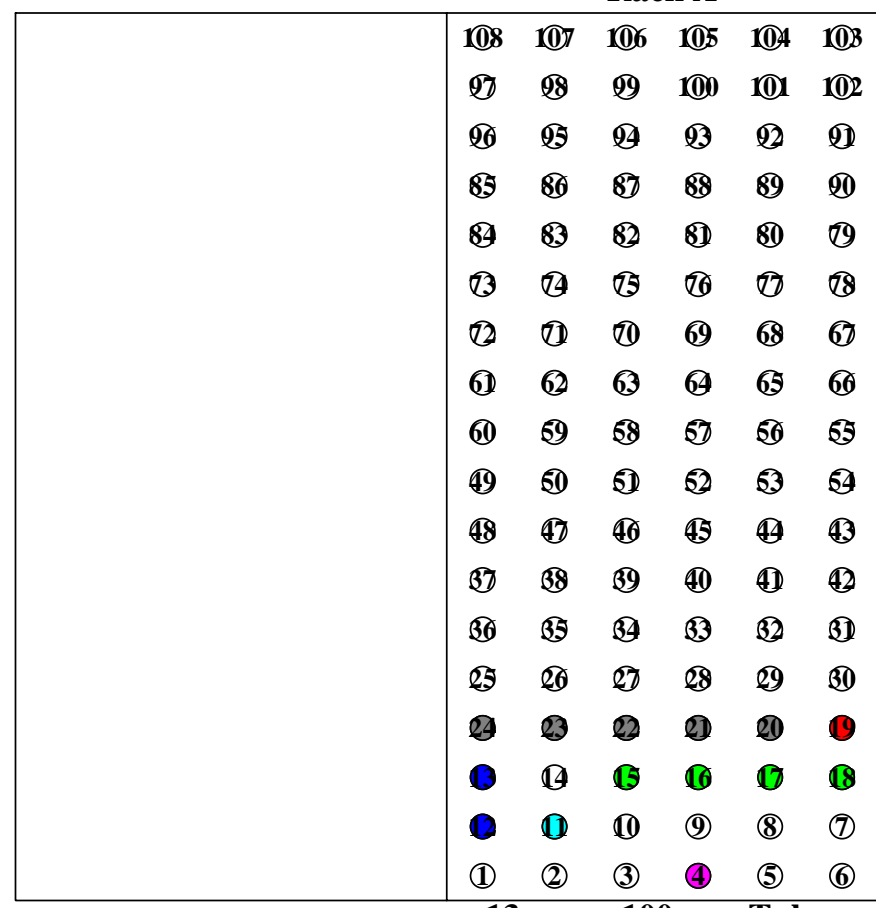

$13 \mathrm{~mm} \times 100 \mathrm{~mm}$ Tubes

\begin{tabular}{|c|c|c|c|}
\hline $\begin{array}{l}1 \\
2 \\
3 \\
4 \\
5 \\
5 \\
6\end{array}$ & & $\begin{array}{c}\text { A:4 } \\
\text { A:11 } \\
\text { A:12 } \\
\text { A:15 } \\
\text { A:19 } \\
\text { A:20 }\end{array}$ & $\begin{array}{c}\text { A:4 } \\
A: 11 \\
A: 13 \\
A: 18 \\
A: 19 \\
A: 24\end{array}$ \\
\hline Duration & $\% \mathrm{~B}$ & Solvent A & Solvent B \\
\hline $\begin{array}{c}0.0 \\
1.0 \\
11.0 \\
2.0 \\
0.0 \\
1.0\end{array}$ & \begin{tabular}{c|}
0.0 \\
0.0 \\
100.0 \\
100.0 \\
0.0 \\
0.0
\end{tabular} & $\begin{array}{l}\text { A1 hexane } \\
\text { A1 hexane } \\
\text { A1 hexane } \\
\text { A1 hexane } \\
\text { A1 hexane } \\
\text { A1 hexane }\end{array}$ & $\begin{array}{l}\text { B1 ethyl acetate } \\
\text { B1 ethyl acetate } \\
\text { B1 ethyl acetate } \\
\text { B1 ethyl acetate } \\
\text { B1 ethyl acetate } \\
\text { B1 ethyl acetate }\end{array}$ \\
\hline
\end{tabular}


Compound [26]

Sample: ALC-B-268

4x Column: 2

RediSep Column: Silica 4g

Flow Rate: $18 \mathrm{ml} / \mathrm{min}$

Equilibration Volume: $33.6 \mathrm{ml}$

Initial Waste: $0.0 \mathrm{ml}$

Air Purge: 10.0 min

Solvent: A1 hexane

Solvent: B1 ethyl acetate

\section{Rf 200}

Monday 28 September 2015 09:13AM

Peak Tube Volume: Max.

Non-Peak Tube Volume: Max.

Loading Type: Solid

Wavelength 1 (red): 254nm

Peak Width: 30 sec

Threshold: 0.20 AU

Wavelength 2 (purple): 280nm
All Wavelength (orange): 200nm - 360nm

Peak Width: 30 sec

Threshold: 0.20 AU

Run Notes:

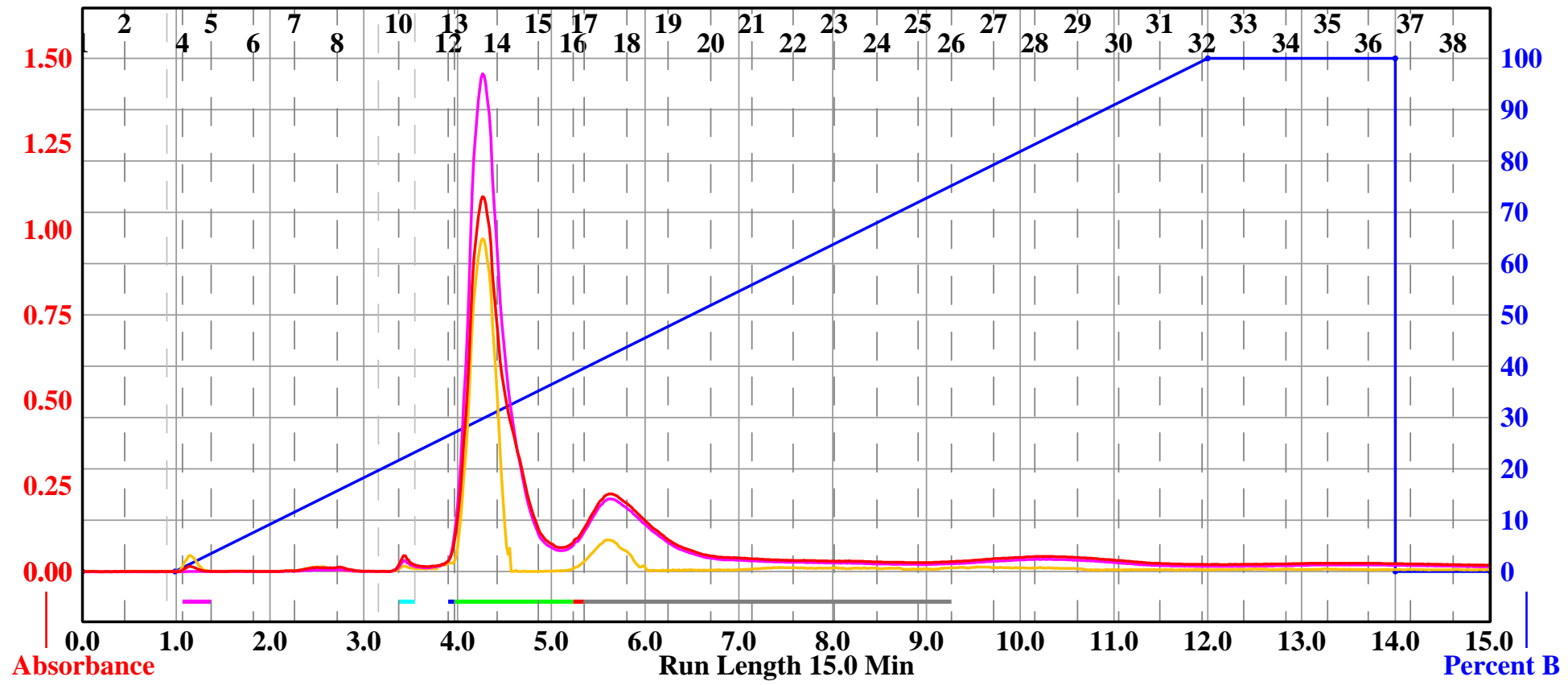

Rack A

\begin{tabular}{|c|c|c|c|c|c|}
\hline 08 & (107 & 106 & 105 & (104 & 103 \\
\hline 17 & 18 & (19) & (100 & (1)1 & 102 \\
\hline 96 & (2) & (9) & (23 & (2) & (1) \\
\hline 35 & 8 & 87 & 88 & 89 & (2) \\
\hline 84 & 83 & 82 & (1) & 80 & (9) \\
\hline 33 & (8) & 18 & (ש) & (77) & 18 \\
\hline 12 & (11) & (11) & (9) & 68 & 67 \\
\hline (1) & (2) & (3) & (4) & 69 & ช6 \\
\hline 1) & 59 & 88 & 57 & b & 5 \\
\hline 49 & (9) & (1) & 52 & 63 & 59 \\
\hline 48 & (47) & (46) & 49 & (4) & (4) \\
\hline (37) & 18 & (39) & (41) & (41) & (42) \\
\hline (Bd) & (39 & B9 & B3 & (32) & (D) \\
\hline 25 & (6) & (28) & 88 & 29 & (10) \\
\hline 9 & 23 & (2) & (2) & (21) & (1) \\
\hline (1) & (1) & (5) & 0 & (1) & 18 \\
\hline 2 & (11) & (10) & (2) & (8) & (7) \\
\hline (1) & (2) & (3) & (4) & (5) & (6) \\
\hline
\end{tabular}

$13 \mathrm{~mm} \times 100 \mathrm{~mm}$ Tubes

\begin{tabular}{|c|c|c|c|}
\hline \multicolumn{2}{|c|}{ Peak \# } & Start Tube & End Tube \\
\hline $\begin{array}{l}1 \\
2 \\
3 \\
4 \\
5 \\
\mathbf{5}\end{array}$ & & $\begin{array}{c}\text { A:4 } \\
\text { A:10 } \\
\text { A:12 } \\
\text { A:13 } \\
\text { A:16 } \\
\text { A:17 }\end{array}$ & $\begin{array}{c}\text { A:4 } \\
\text { A:10 } \\
\text { A:12 } \\
\text { A:15 } \\
\text { A:16 } \\
\text { A:25 }\end{array}$ \\
\hline Duration & $\% \mathbf{B}$ & Solvent A & Solvent B \\
\hline $\begin{array}{c}0.0 \\
1.0 \\
11.0 \\
2.0 \\
0.0 \\
1.0\end{array}$ & \begin{tabular}{|c|}
0.0 \\
0.0 \\
100.0 \\
100.0 \\
0.0 \\
0.0
\end{tabular} & $\begin{array}{l}\text { A1 hexane } \\
\text { A1 hexane } \\
\text { A1 hexane } \\
\text { A1 hexane } \\
\text { A1 hexane } \\
\text { A1 hexane }\end{array}$ & $\begin{array}{l}\text { B1 ethyl acetate } \\
\text { B1 ethyl acetate } \\
\text { B1 ethyl acetate } \\
\text { B1 ethyl acetate } \\
\text { B1 ethyl acetate } \\
\text { B1 ethyl acetate }\end{array}$ \\
\hline
\end{tabular}


Compound [27]

Sample: ALC-C-46

4x Column: 2

RediSep Column: Silica $4 \mathrm{~g}$

Flow Rate: $18 \mathrm{ml} / \mathrm{min}$

Equilibration Volume: $33.6 \mathrm{ml}$

Initial Waste: $0.0 \mathrm{ml}$

Air Purge: 10.0 min

Solvent: A1 hexane

Solvent: B1 ethyl acetate

\section{Rf 200}

Peak Tube Volume: Max.

Non-Peak Tube Volume: Max.

Loading Type: Solid

Wavelength 1 (red): 254nm

Peak Width: $30 \mathrm{sec}$

Threshold: 0.20 AU

Wavelength 2 (purple): $280 \mathrm{~nm}$
Wednesday 14 October 2015 09:08AM

All Wavelength (orange): 200nm - 360nm

Peak Width: 30 sec

Threshold: 0.20 AU

Run Notes:

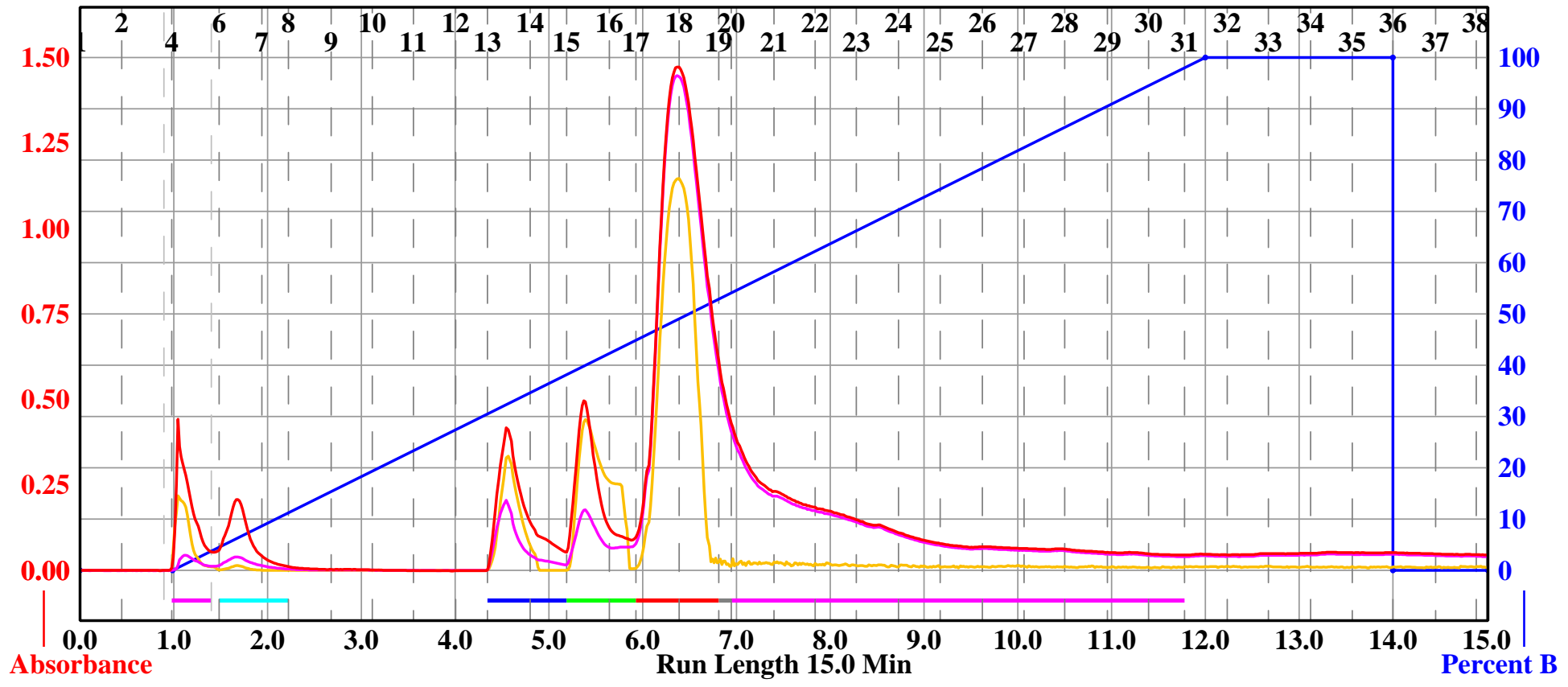

Rack A

\begin{tabular}{|c|c|c|c|c|c|}
\hline & (107 & 106 & 105 & (104) & 103 \\
\hline 87 & 18 & (9) & (100 & (1) & (102 \\
\hline 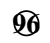 & (צ & (9) & (2) & (2) & (1) \\
\hline 38 & 8 & 87 & 88 & 89 & (2) \\
\hline 9 & 83 & 82 & 80 & (81) & (19) \\
\hline 13 & (8) & (18) & (8) & (77) & 18 \\
\hline (2) & (11) & (11) & (9) & 68 & (67) \\
\hline (1) & (2) & (3) & (4) & 65) & (6) \\
\hline (1) & (99 & 68 & 57 & ஒd & (5) \\
\hline 49 & $\mathfrak{b}$ & (1) & 52 & 53 & 59 \\
\hline 48 & (47) & (46) & (4) & (4) & 43 \\
\hline (37) & 18 & (99) & (40) & (47) & (42) \\
\hline WO & BS & B & B3 & (32) & (1) \\
\hline 25 & 6 & 87 & 8 & (2) & (2) \\
\hline 28 & 8 & (2) & (D) & (21) & (1) \\
\hline & 0 & (1) & (6) & 0 & (1) \\
\hline (12) & (1) & (11) & (D) & (8) & (7) \\
\hline (1) & (2) & (3) & (4) & (5) & (6) \\
\hline
\end{tabular}

$13 \mathrm{~mm} \times 100 \mathrm{~mm}$ Tubes

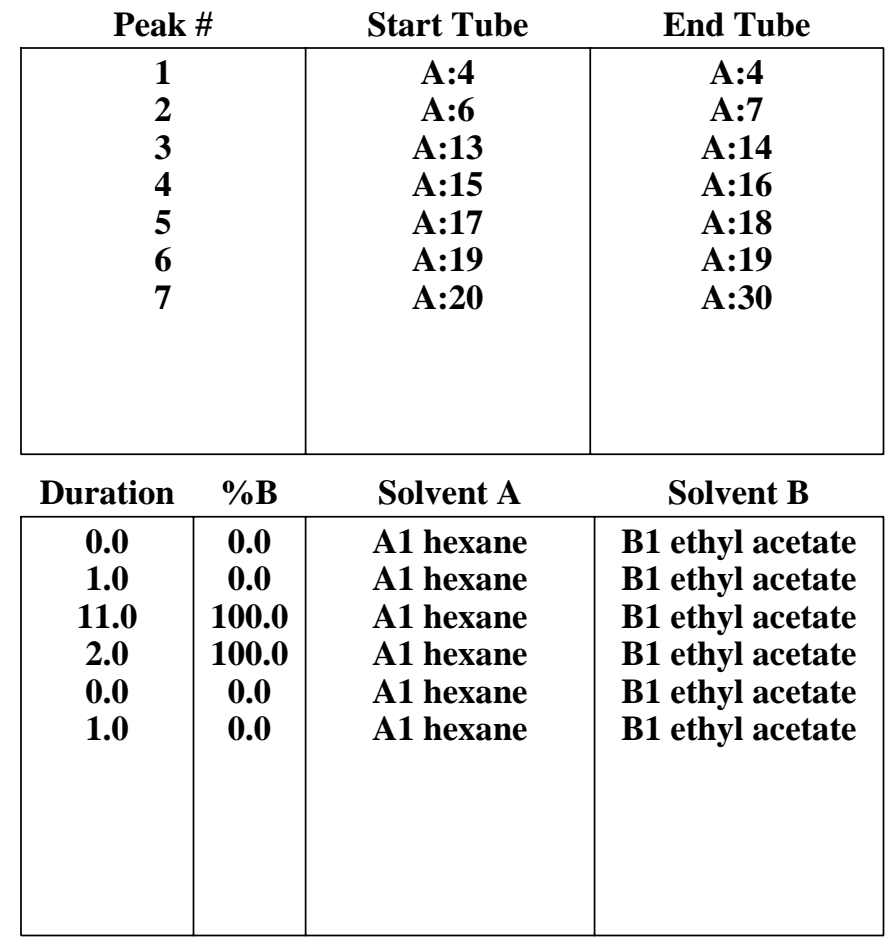


Compound [28]

Sample: ALC-C-58

4x Column: 2

RediSep Column: Silica 4g

Flow Rate: $18 \mathrm{ml} / \mathrm{min}$

Equilibration Volume: $33.6 \mathrm{ml}$

Initial Waste: $0.0 \mathrm{ml}$

Air Purge: 10.0 min

Solvent: A1 hexane

Solvent: B1 ethyl acetate

\section{Rf 200}

Thursday 15 October 2015 12:52PM

Peak Tube Volume: Max.

Non-Peak Tube Volume: Max.

Loading Type: Solid

Wavelength 1 (red): 254nm

Peak Width: $30 \mathrm{sec}$

Threshold: 0.20 AU

Wavelength 2 (purple): 280nm
All Wavelength (orange): 200nm - 360nm

Peak Width: $30 \mathrm{sec}$

Threshold: 0.20 AU

Run Notes:

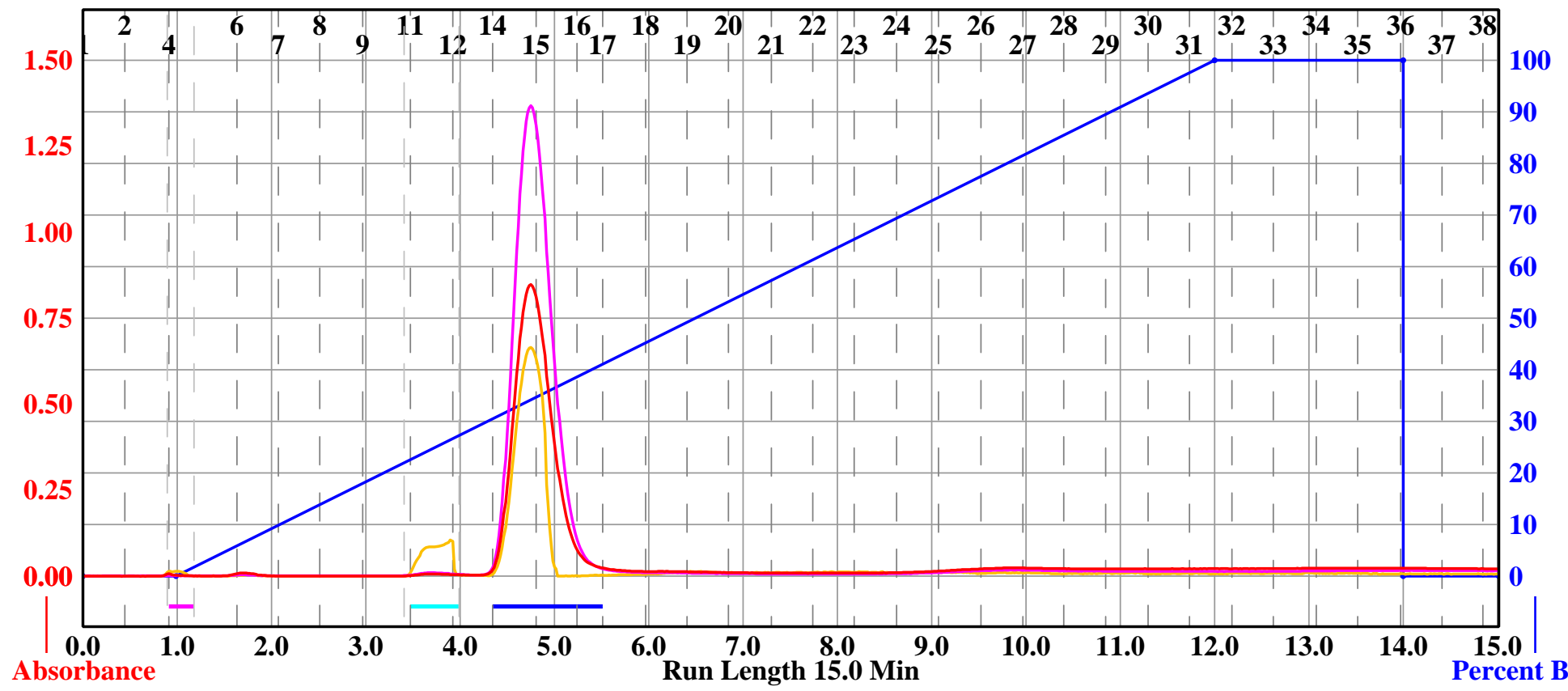

Rack A

Peak \#

Start Tube

End Tube

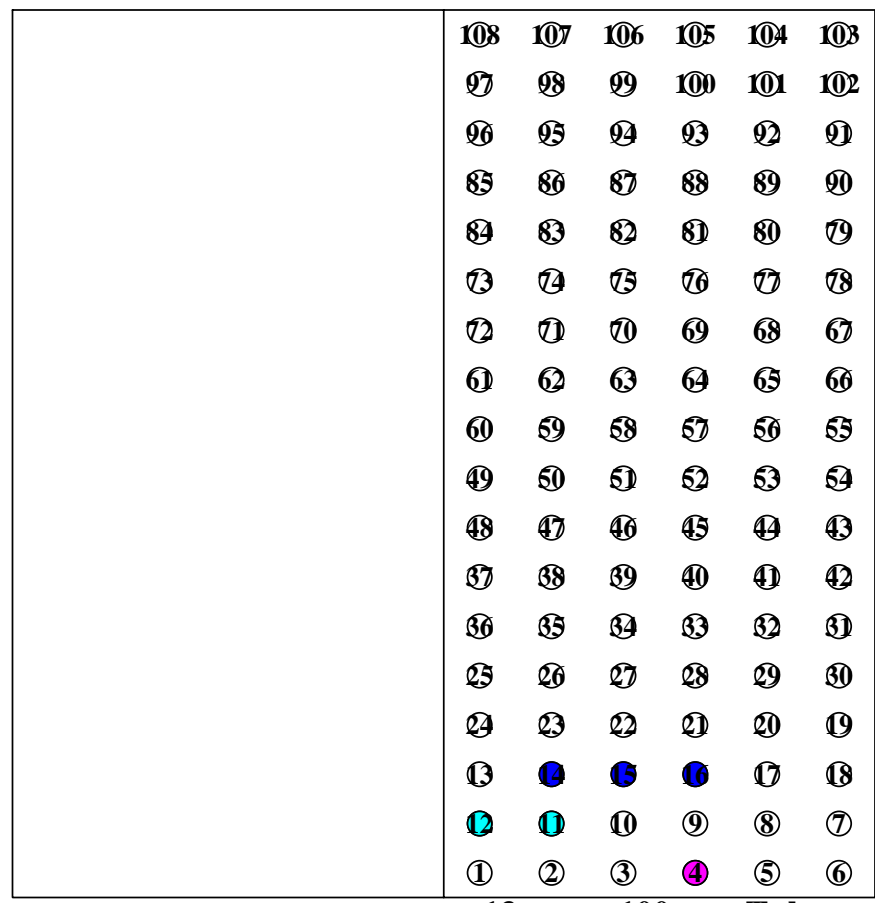

$13 \mathrm{~mm} \times 100 \mathrm{~mm}$ Tubes

\begin{tabular}{|c|c|c|c|}
\hline $\begin{array}{l}1 \\
2 \\
3\end{array}$ & & $\begin{array}{c}\text { A:4 } \\
\text { A:11 } \\
\text { A:14 }\end{array}$ & $\begin{array}{c}A: 4 \\
A: 12 \\
A: 16\end{array}$ \\
\hline Duration & $\% \mathbf{B}$ & Solvent A & Solvent B \\
\hline $\begin{array}{c}0.0 \\
1.0 \\
11.0 \\
2.0 \\
0.0 \\
1.0\end{array}$ & \begin{tabular}{|c|}
0.0 \\
0.0 \\
100.0 \\
100.0 \\
0.0 \\
0.0
\end{tabular} & $\begin{array}{l}\text { A1 hexane } \\
\text { A1 hexane } \\
\text { A1 hexane } \\
\text { A1 hexane } \\
\text { A1 hexane } \\
\text { A1 hexane }\end{array}$ & $\begin{array}{l}\text { B1 ethyl acetate } \\
\text { B1 ethyl acetate } \\
\text { B1 ethyl acetate } \\
\text { B1 ethyl acetate } \\
\text { B1 ethyl acetate } \\
\text { B1 ethyl acetate }\end{array}$ \\
\hline
\end{tabular}


Compound [29]

Sample: ALC-B-280

4x Column: 2

RediSep Column: Silica $4 \mathrm{~g}$

Flow Rate: $18 \mathrm{ml} / \mathrm{min}$

Equilibration Volume: $33.6 \mathrm{ml}$

Initial Waste: $0.0 \mathrm{ml}$

Air Purge: 10.0 min

Solvent: A1 hexane

Solvent: B1 ethyl acetate

\section{Rf 200}

Friday 02 October 2015 11:28AM

Peak Tube Volume: Max.

Non-Peak Tube Volume: Max.

Loading Type: Solid

Wavelength 1 (red): 254nm

Peak Width: 30 sec

Threshold: 0.20 AU

Wavelength 2 (purple): 280nm
All Wavelength (orange): 200nm - 360nm

Peak Width: $30 \mathrm{sec}$

Threshold: 0.20 AU

Run Notes:

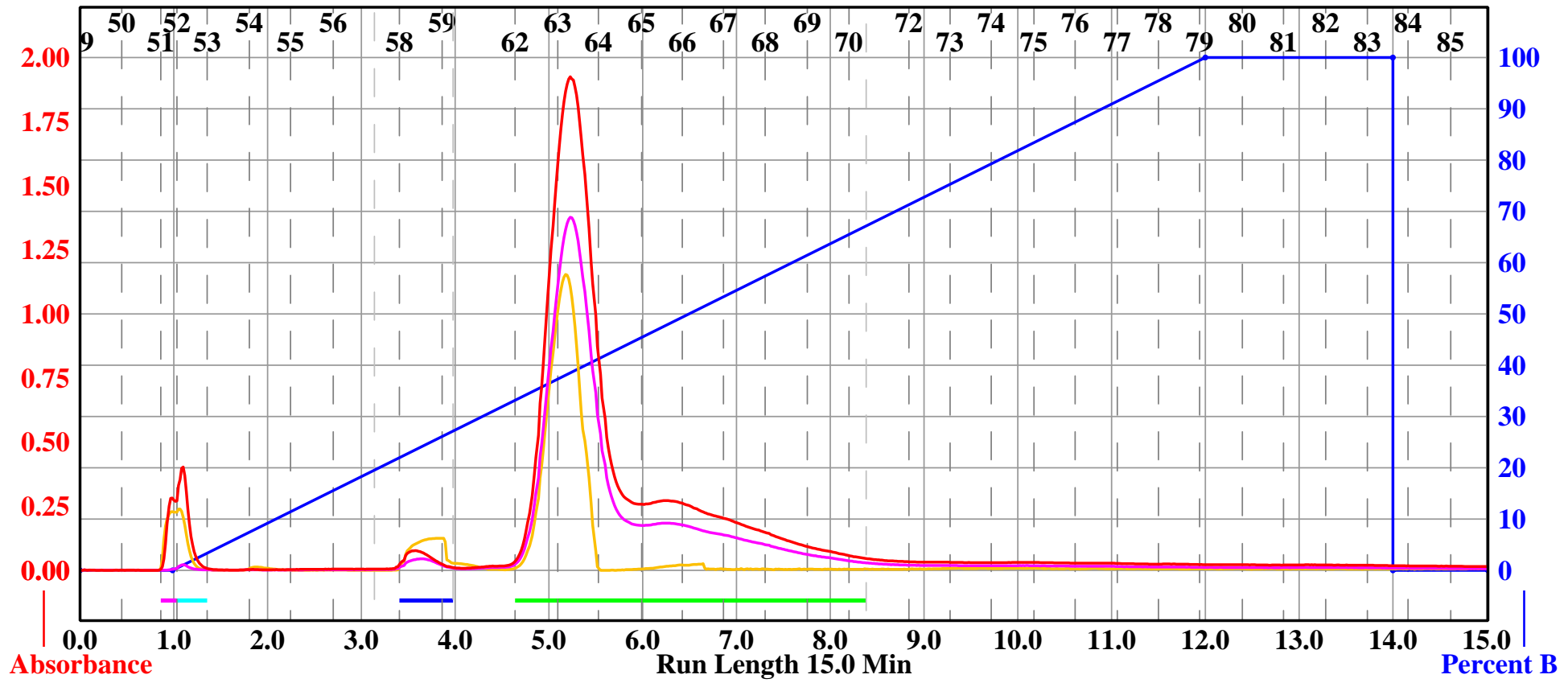

Rack A

Peak \#

Start Tube

End Tube

\begin{tabular}{|c|c|c|c|c|c|}
\hline 18 & (107) & 106 & 105 & (10) & (103 \\
\hline 27 & $\mathscr{O B}$ & (9) & 100 & (1) & $1(102$ \\
\hline Vு & (2) & (צ) & $\mathscr{2}$ & (22) & (1) \\
\hline 35 & 8 & 87 & 88 & 89 & (2) \\
\hline 89 & 83 & 82 & 80 & 80 & (19) \\
\hline 13 & 87 & 18 & (ש) & (17) & 88 \\
\hline (2) & (1) & (11) & (9) & 68 & 67 \\
\hline (1) & (2) & (3) & (4) & 6 & (6) \\
\hline (10) & (2) & (ㄱ) & 58 & ஐ & 5 \\
\hline 49 & (10) & (2) & (2) & 53 & 59 \\
\hline 48 & (47) & (4) & 48 & (4) & 43 \\
\hline (37) & 18 & (9) & (41) & (47) & (42) \\
\hline bo & WG & B9 & B3 & (22) & (1D) \\
\hline (25) & 26 & 87 & 88 & (2) & (10) \\
\hline (2) & 23 & (2) & (2) & (21) & (1) \\
\hline (1) & (1) & (15) & (16) & (17) & 18 \\
\hline (12) & (11) & (1) & (D) & (8) & (7) \\
\hline (1) & (2) & (3) & (4) & (5) & (6) \\
\hline
\end{tabular}

$13 \mathrm{~mm} \times 100 \mathrm{~mm}$ Tubes

\begin{tabular}{|c|c|c|c|}
\hline $\begin{array}{l}\mathbf{1} \\
\mathbf{2} \\
\mathbf{3} \\
\mathbf{4}\end{array}$ & & $\begin{array}{l}\text { A:51 } \\
\text { A:52 } \\
\text { A:58 } \\
\text { A:62 }\end{array}$ & $\begin{array}{l}\text { A:51 } \\
\text { A:52 } \\
\text { A:59 } \\
\text { A:70 }\end{array}$ \\
\hline Duration & $\% \mathbf{B}$ & Solvent A & Solvent B \\
\hline $\begin{array}{c}\text { 0.0 } \\
1.0 \\
11.0 \\
2.0 \\
0.0 \\
1.0\end{array}$ & \begin{tabular}{|c|}
0.0 \\
0.0 \\
100.0 \\
100.0 \\
0.0 \\
0.0
\end{tabular} & $\begin{array}{l}\text { A1 hexane } \\
\text { A1 hexane } \\
\text { A1 hexane } \\
\text { A1 hexane } \\
\text { A1 hexane } \\
\text { A1 hexane }\end{array}$ & $\begin{array}{l}\text { B1 ethyl acetate } \\
\text { B1 ethyl acetate } \\
\text { B1 ethyl acetate } \\
\text { B1 ethyl acetate } \\
\text { B1 ethyl acetate } \\
\text { B1 ethyl acetate }\end{array}$ \\
\hline
\end{tabular}


Compound [30]

Sample: ALC-B-244

4x Column: 2

RediSep Column: Silica 4g

Flow Rate: $18 \mathrm{ml} / \mathrm{min}$

Equilibration Volume: $33.6 \mathrm{ml}$

Initial Waste: $0.0 \mathrm{ml}$

Air Purge: 10.0 min

Solvent: A1 hexane

Solvent: B2 methanol

\section{Rf 200}

Friday 25 September 2015 09:50AM

Peak Tube Volume: Max.

Non-Peak Tube Volume: Max.

Loading Type: Solid

Wavelength 1 (red): 254nm

Peak Width: 30 sec

Threshold: 0.20 AU

Wavelength 2 (purple): 280nm
All Wavelength (orange): 200nm - 360nm

Peak Width: 30 sec

Threshold: 0.20 AU

Run Notes:

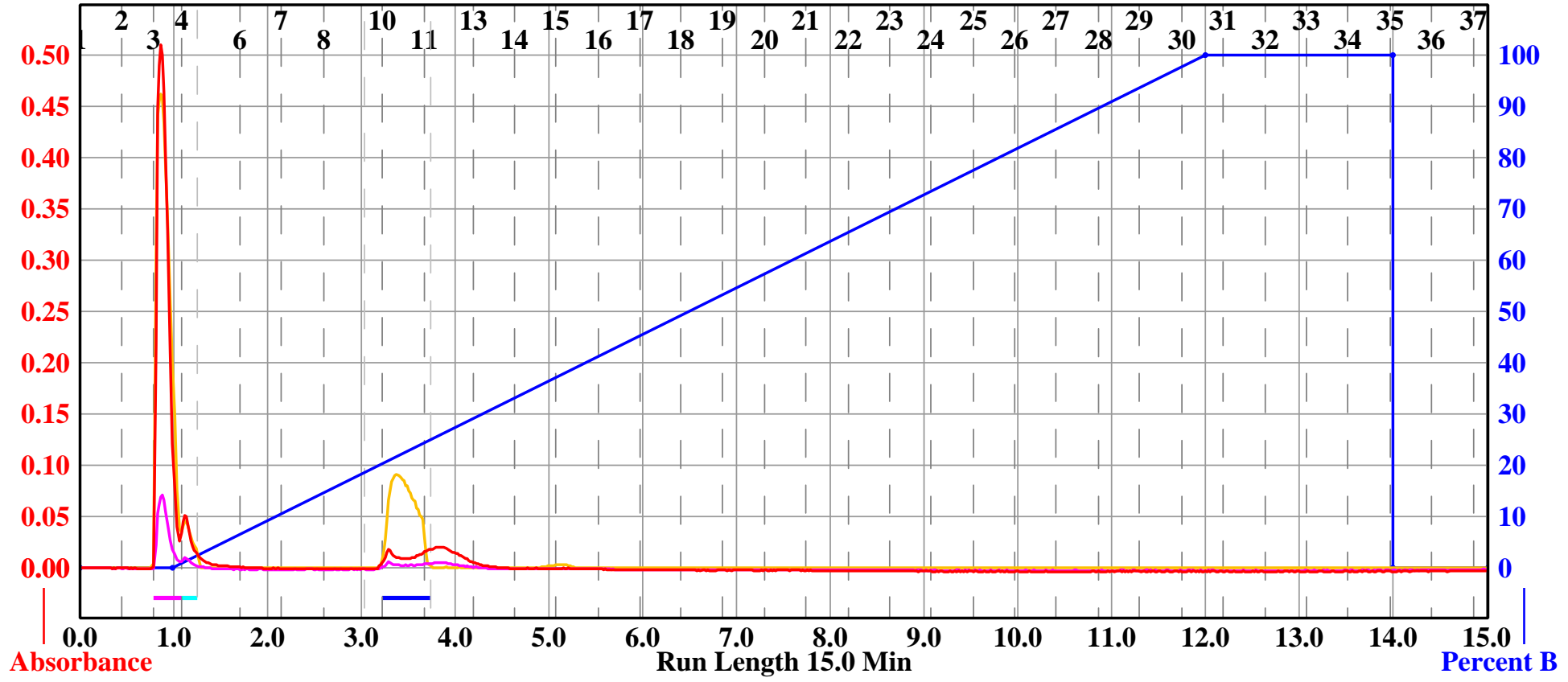

\begin{tabular}{|c|c|c|c|c|c|}
\hline \multicolumn{6}{|c|}{ Rack A } \\
\hline & (107 & 106 & 105 & (104 & (10) \\
\hline 27 & $\mathscr{B}$ & (9) & 100 & (10) & (1) \\
\hline V6 & (1) & $\mathscr{P}$ & Q3 & (2) & (2) \\
\hline 85 & 8 & 87 & 88 & 89 & (2) \\
\hline 89 & 8 & 82 & 80 & (10) & (19) \\
\hline 13 & (8) & (15) & (20) & (17) & 88 \\
\hline 12 & (11) & (10) & (99) & 68 & 67 \\
\hline (1) & (2) & (3) & 6 & 6 & (6) \\
\hline (1) & 59 & 68 & (57) & ๒ & 55 \\
\hline (4) & (9) & (1) & 52 & 53 & 5 \\
\hline 48 & (47) & (40) & (45) & (4) & 43 \\
\hline (37) & $B 8$ & (39) & (41) & (41) & (42) \\
\hline b & BS & (3) & B3 & (32) & (1) \\
\hline 25 & שd & (27) & 8 & (9) & (10) \\
\hline 29 & 23 & (2) & (1) & (21) & (19) \\
\hline (13) & (1) & (15) & (16) & (17) & (18 \\
\hline (12) & 0 & - & (2) & (8) & (7) \\
\hline (1) & (2) & (3) & (4) & (5) & (6) \\
\hline
\end{tabular}

$13 \mathrm{~mm} \times 100 \mathrm{~mm}$ Tubes

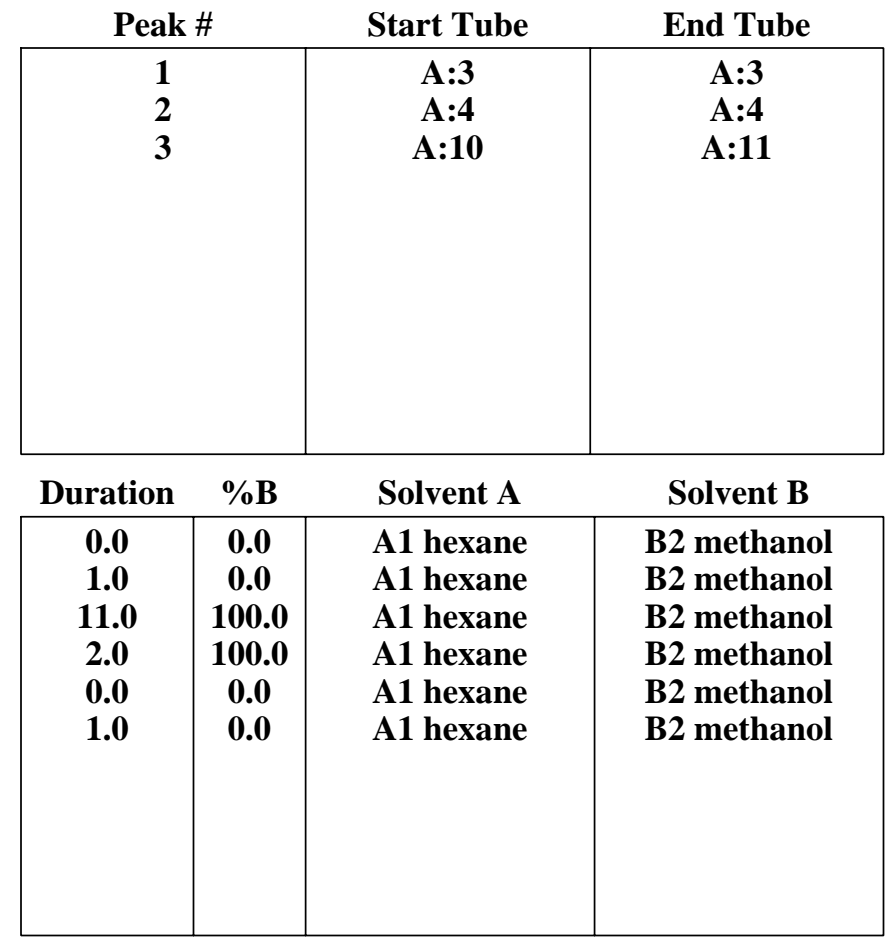


4x Column: 2

RediSep Column: Silica 4g

Flow Rate: $18 \mathrm{ml} / \mathrm{min}$

Equilibration Volume: $33.6 \mathrm{ml}$

Initial Waste: $0.0 \mathrm{ml}$

Air Purge: 10.0 min

Solvent: A1 hexane

Solvent: B2 methanol
Peak Tube Volume: Max.

Non-Peak Tube Volume: Max.

Loading Type: Solid

Wavelength 1 (red): 254nm

Peak Width: 30 sec

Threshold: 0.20 AU

Wavelength 2 (purple): 280nm
All Wavelength (orange): 200nm - 360nm

Peak Width: 30 sec

Threshold: 0.20 AU

Run Notes:

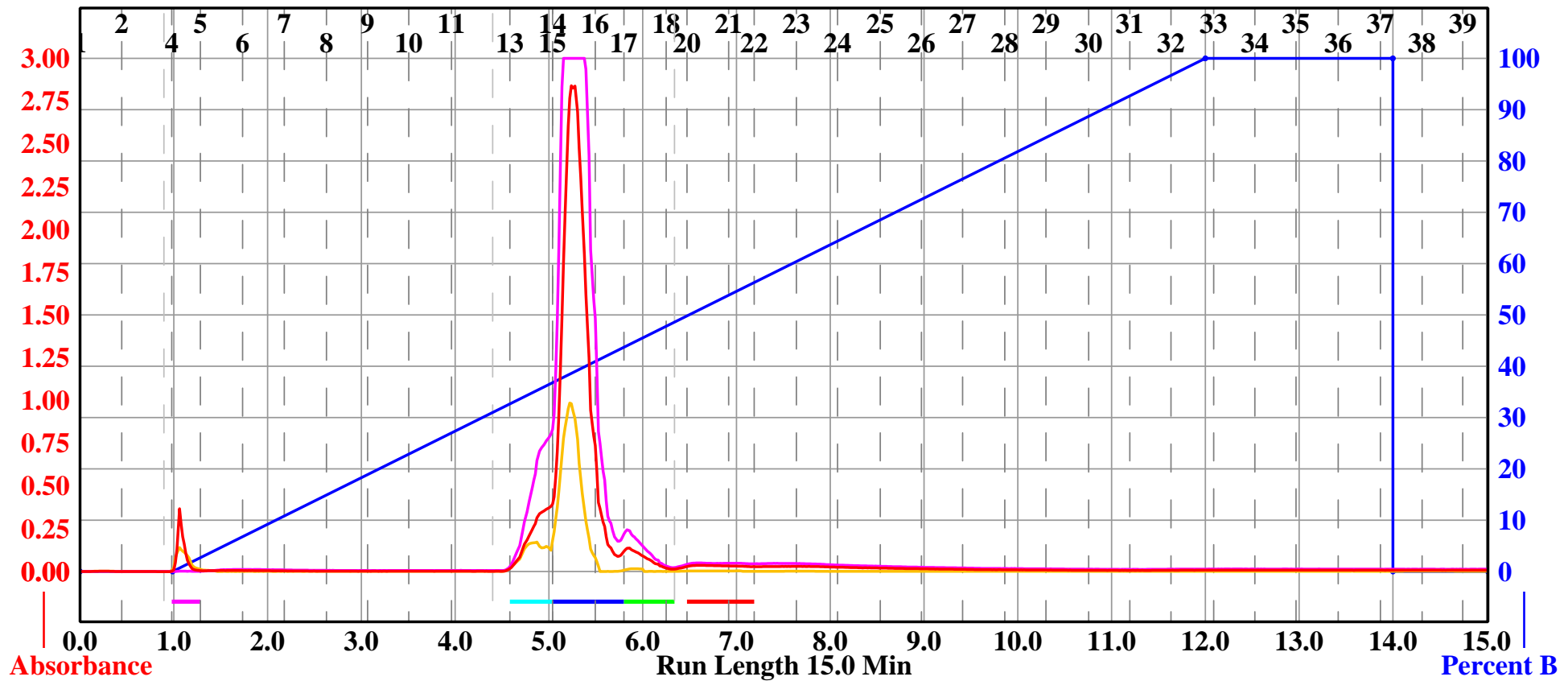

Rack A

\begin{tabular}{|c|c|c|c|c|c|}
\hline & (107) & 106 & 105 & (104 & 103 \\
\hline 27 & $\mathscr{B}$ & (9) & 100 & (1) & 102 \\
\hline 26 & (2) & (A) & 19 & (2) & (2) \\
\hline 35 & 8 & 87 & 88 & 89 & (2) \\
\hline 39 & 83 & 82 & 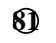 & 80 & (19) \\
\hline 13 & (8) & (15) & (20) & (17) & 18 \\
\hline (2) & (1) & (10) & (99) & 68 & (67) \\
\hline (1) & (2) & (3) & (4) & (6) & ஏ \\
\hline (10) & 59 & 68 & 57 & ஒ & 57 \\
\hline (49) & (D) & (1) & 52 & 53 & 59 \\
\hline 48 & (47) & (46) & 49 & (4) & 43 \\
\hline (37) & B8 & (99) & (41) & (47) & (42) \\
\hline bd & BS & B & B3 & (32) & (1) \\
\hline 25 & שூ & (28) & 8 & (29) & (10) \\
\hline (2) & 23 & 22 & D & (2) & (19 \\
\hline (1) & (1) & 0 & 0 & (1) & 18 \\
\hline (12) & (11) & (1) & (2) & (8) & (7) \\
\hline (1) & (2) & (3) & (4) & (5) & (6) \\
\hline
\end{tabular}

$13 \mathrm{~mm} \times 100 \mathrm{~mm}$ Tubes

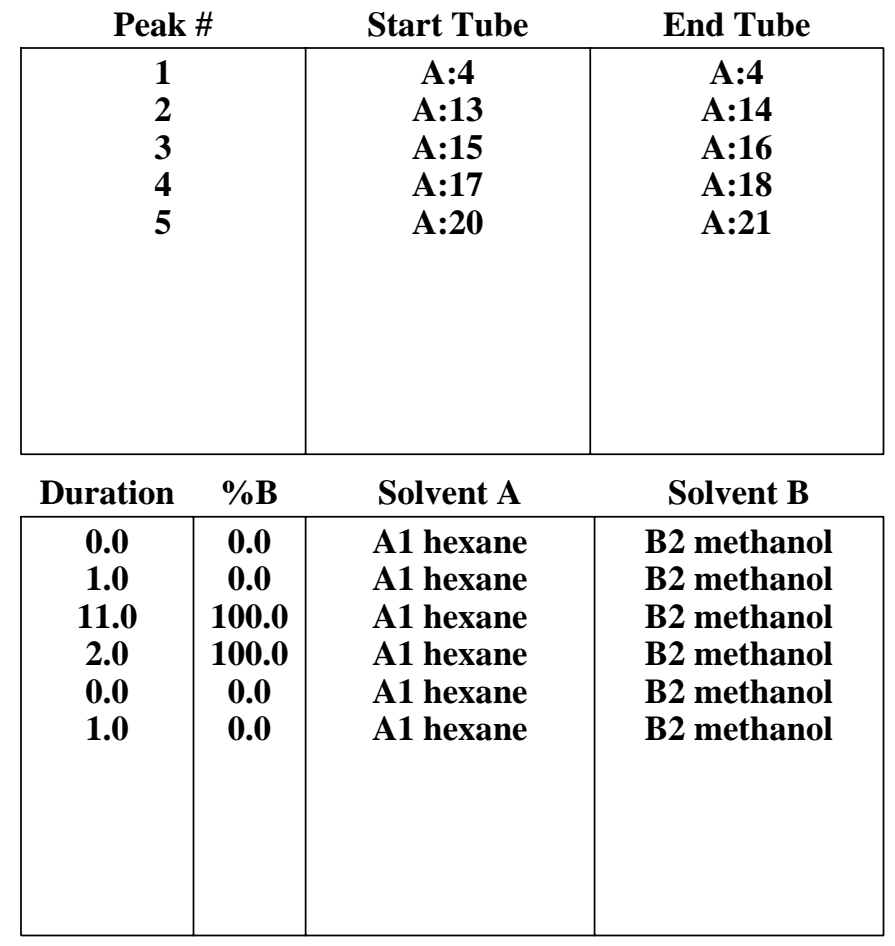


Compound [32]

Sample: ALC-B-272

4x Column: 2

RediSep Column: Silica 4g

Flow Rate: $18 \mathrm{ml} / \mathrm{min}$

Equilibration Volume: $33.6 \mathrm{ml}$

Initial Waste: $0.0 \mathrm{ml}$

Air Purge: 10.0 min

Solvent: A1 hexane

Solvent: B2 methanol

\section{Rf 200}

Thursday 01 October 2015 12:34PM

Peak Tube Volume: Max.

Non-Peak Tube Volume: Max.

Loading Type: Solid

Wavelength 1 (red): 254nm

Peak Width: 30 sec

Threshold: 0.20 AU

Wavelength 2 (purple): 280nm
All Wavelength (orange): $200 \mathrm{~nm}-360 \mathrm{~nm}$

Peak Width: $30 \mathrm{sec}$

Threshold: 0.20 AU

Run Notes:

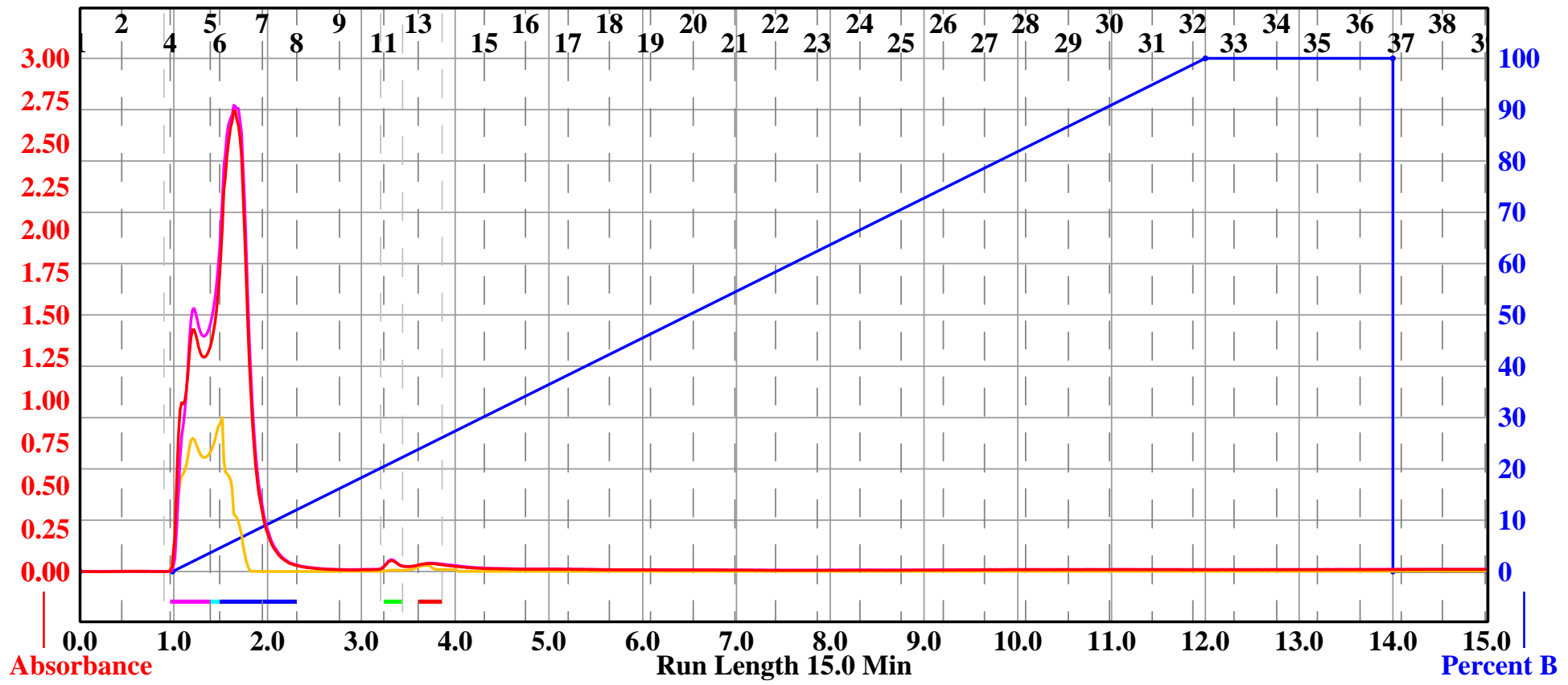

\begin{tabular}{|c|c|c|c|c|c|}
\hline \multicolumn{6}{|c|}{ Rack A } \\
\hline & 107 & (106 & 105 & (104 & \\
\hline & $\mathscr{B}$ & (9) & (100 & (10) & \\
\hline (2) & (1) & (9) & Q3 & (2) & 9 \\
\hline 15 & 8 & 87 & 88 & 89 & \\
\hline & 83 & 82 & 80 & (810 & \\
\hline$(3)$ & (8) & $(15$ & (20 & (17) & \\
\hline 4 & (1) & (10) & (99) & 68 & 6 \\
\hline (1) & (2) & (3) & (4) & 67 & \\
\hline (10 & 5 & 68 & 57 & ש & 5 \\
\hline (49) & (10) & (D) & 52 & 53 & 5 \\
\hline 48 & (47) & 40 & 45 & 49 & \\
\hline (37) & $B 8$ & (39) & (41) & (41) & (4) \\
\hline Bd & (3) & B9 & B & (32) & \\
\hline 25 & (d) & 82 & 8 & 9 & 3 \\
\hline (2) & 23 & 22 & (1) & (2) & ( \\
\hline (1) & (1) & (1) & (16) & 17 & \\
\hline (12) & (1) & (1) & (D) & (8) & \\
\hline (1) & (2) & (3) & (4) & (5) & \\
\hline
\end{tabular}

$13 \mathrm{~mm} \times 100 \mathrm{~mm}$ Tubes

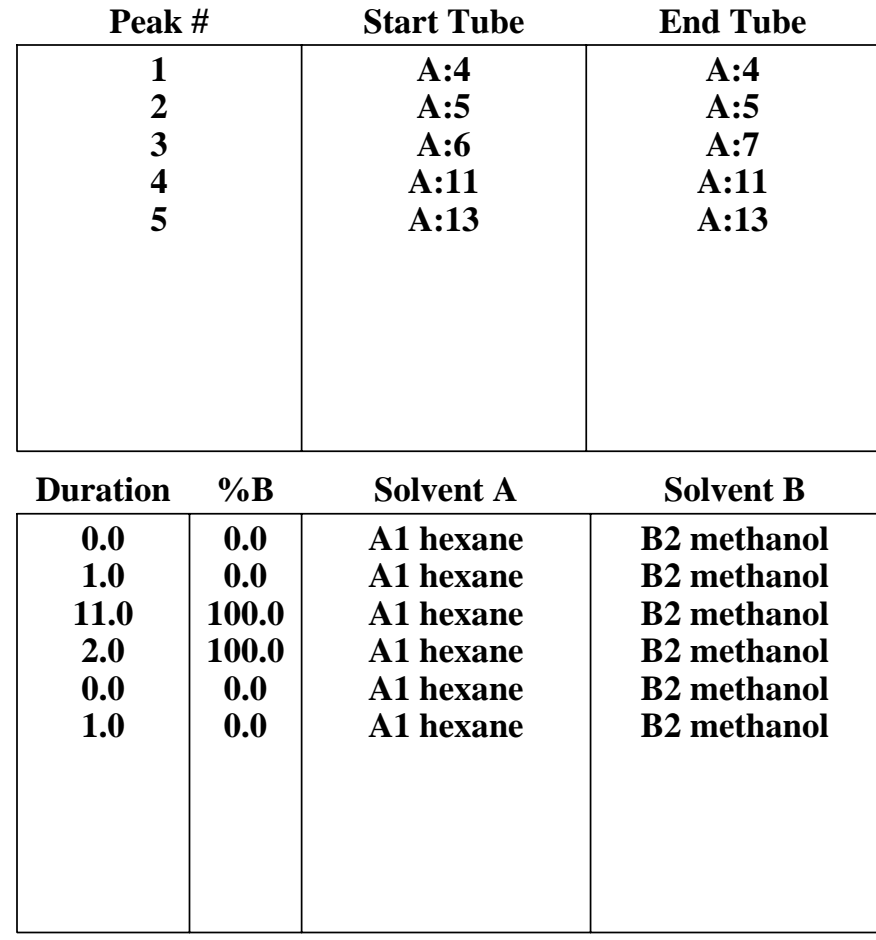


Compound [34]

Sample: ALC-C-38

4x Column: 2

RediSep Column: Silica 4g

Flow Rate: $18 \mathrm{ml} / \mathrm{min}$

Equilibration Volume: $33.6 \mathrm{ml}$

Initial Waste: $0.0 \mathrm{ml}$

Air Purge: 10.0 min

Solvent: A1 hexane

Solvent: B1 ethyl acetate

\section{Rf 200}

Peak Tube Volume: Max.

Non-Peak Tube Volume: Max.

Loading Type: Solid

Wavelength 1 (red): 254nm

Peak Width: $30 \mathrm{sec}$

Threshold: 0.20 AU

Wavelength 2 (purple): 280nm
Wednesday 07 October 2015 01:49PM

All Wavelength (orange): $200 \mathrm{~nm}-360 \mathrm{~nm}$

Peak Width: $30 \mathrm{sec}$

Threshold: 0.20 AU

Run Notes:

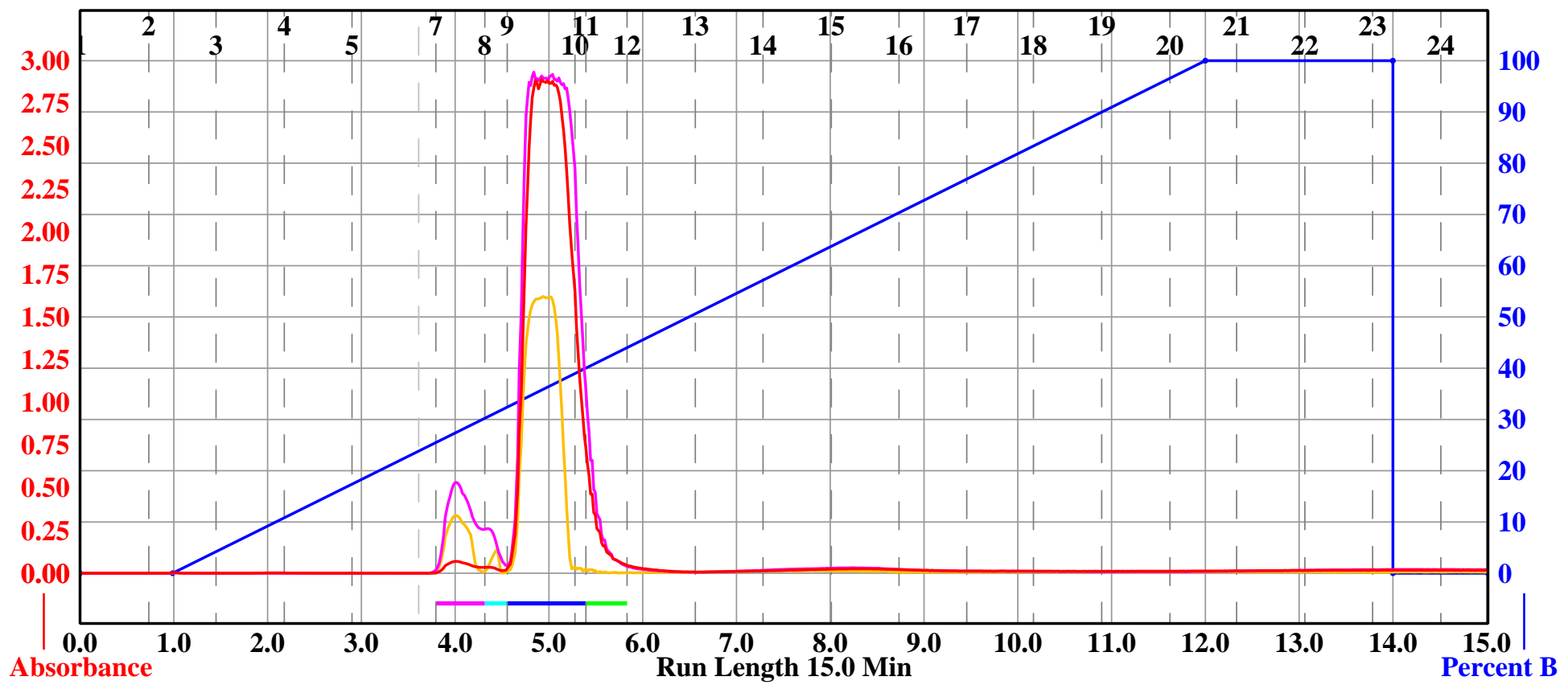

Rack A

\begin{tabular}{|c|c|c|c|}
\hline (11) & (72) & (73) & (74) \\
\hline (70) & (69) & (68) & (67) \\
\hline (11) & (62) & (63) & (44) \\
\hline (60) & (99) & (58) & (57) \\
\hline (51) & (52) & (33) & (54) \\
\hline (50) & (49) & (48) & (47) \\
\hline (41) & (42) & (43) & (44) \\
\hline (40) & (39) & (38) & (37) \\
\hline (31) & (32) & (33) & (34) \\
\hline (30) & (29) & (28) & (27) \\
\hline (21) & (22) & (23) & (24) \\
\hline (20) & (19) & (18) & (17) \\
\hline (11) & (12) & (13) & (14) \\
\hline & & (8) & (7) \\
\hline (1) & (2) & (3) & (4) \\
\hline
\end{tabular}

$16 \mathrm{~mm} \times 100 \mathrm{~mm}$ Tubes

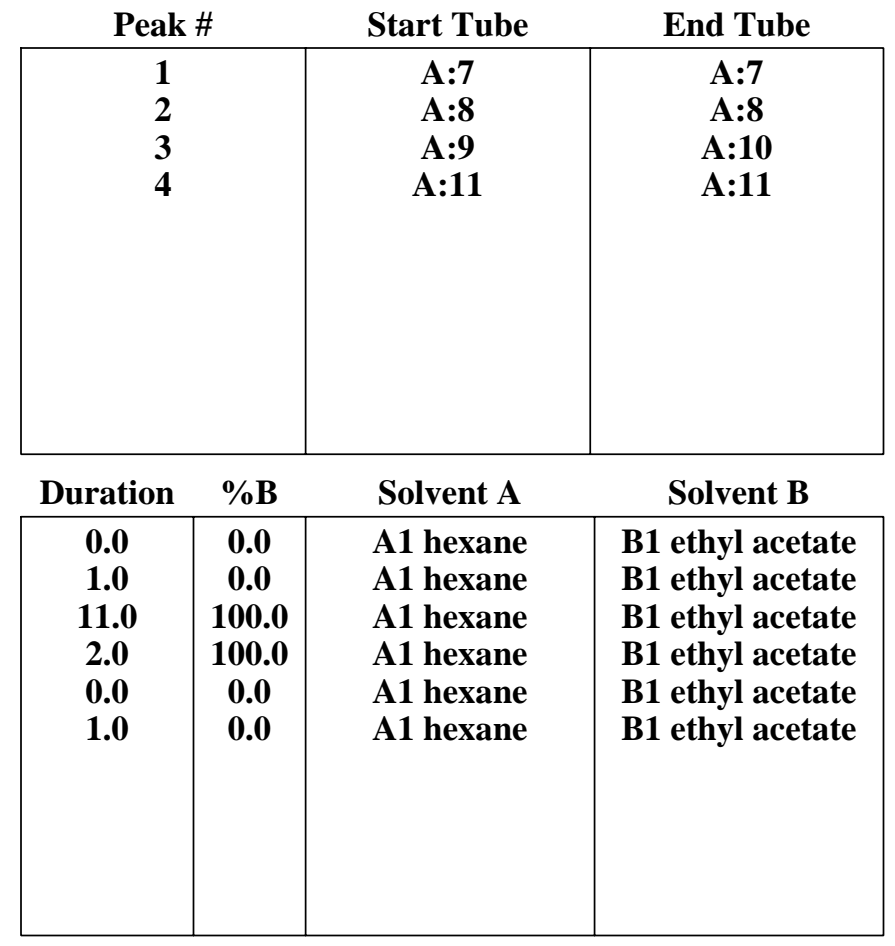


Compound [35]

Sample: ALC-C-34

4x Column: 3

RediSep Column: Silica 4g

Flow Rate: $18 \mathrm{ml} / \mathrm{min}$

Equilibration Volume: $33.6 \mathrm{ml}$

Initial Waste: $0.0 \mathrm{ml}$

Air Purge: 10.0 min

Solvent: A1 hexane

Solvent: B1 ethyl acetate

\section{Rf 200}

Peak Tube Volume: Max.

Non-Peak Tube Volume: Max.

Loading Type: Solid

Wavelength 1 (red): 254nm

Peak Width: $30 \mathrm{sec}$

Threshold: 0.20 AU

Wavelength 2 (purple): 280nm
Wednesday 07 October 2015 02:19PM

All Wavelength (orange): $200 \mathrm{~nm}-360 \mathrm{~nm}$

Peak Width: $30 \mathrm{sec}$

Threshold: 0.20 AU

Run Notes:

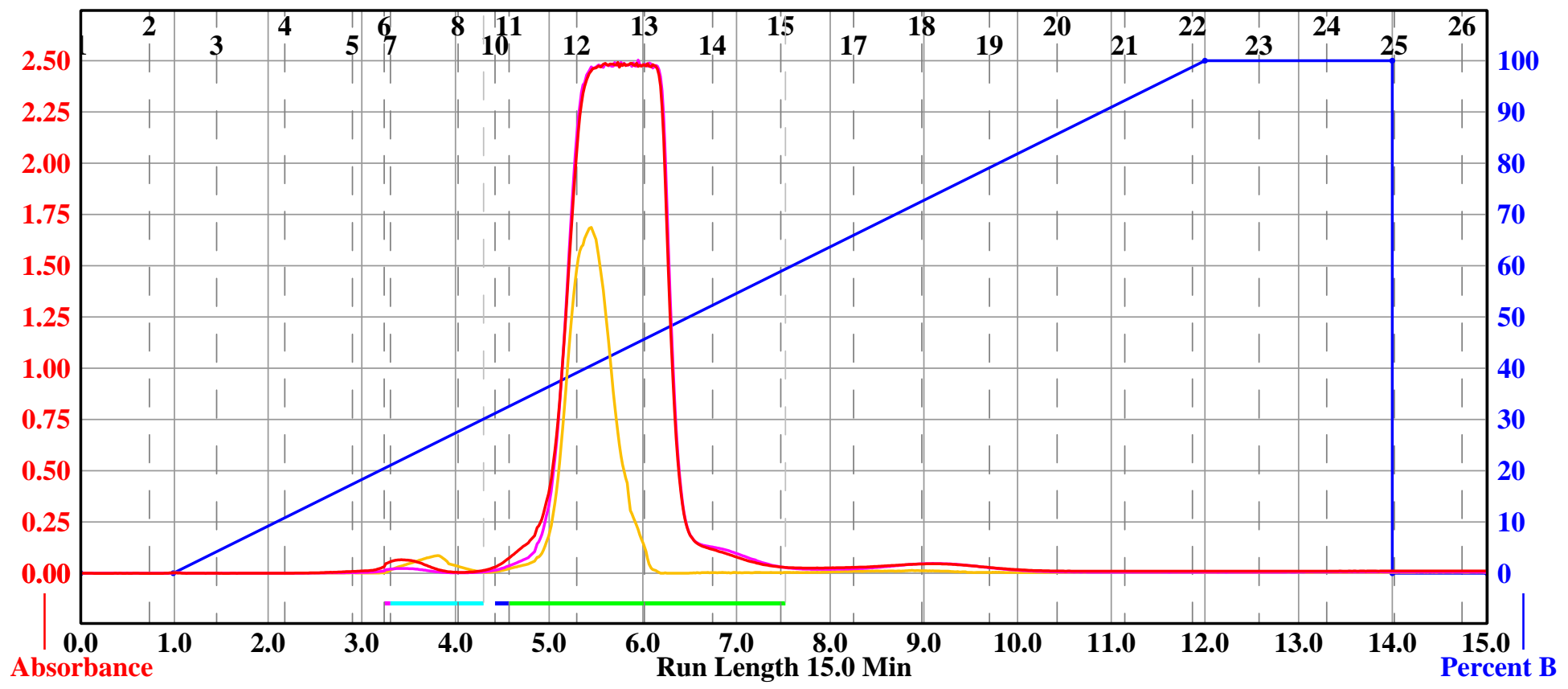

Rack A

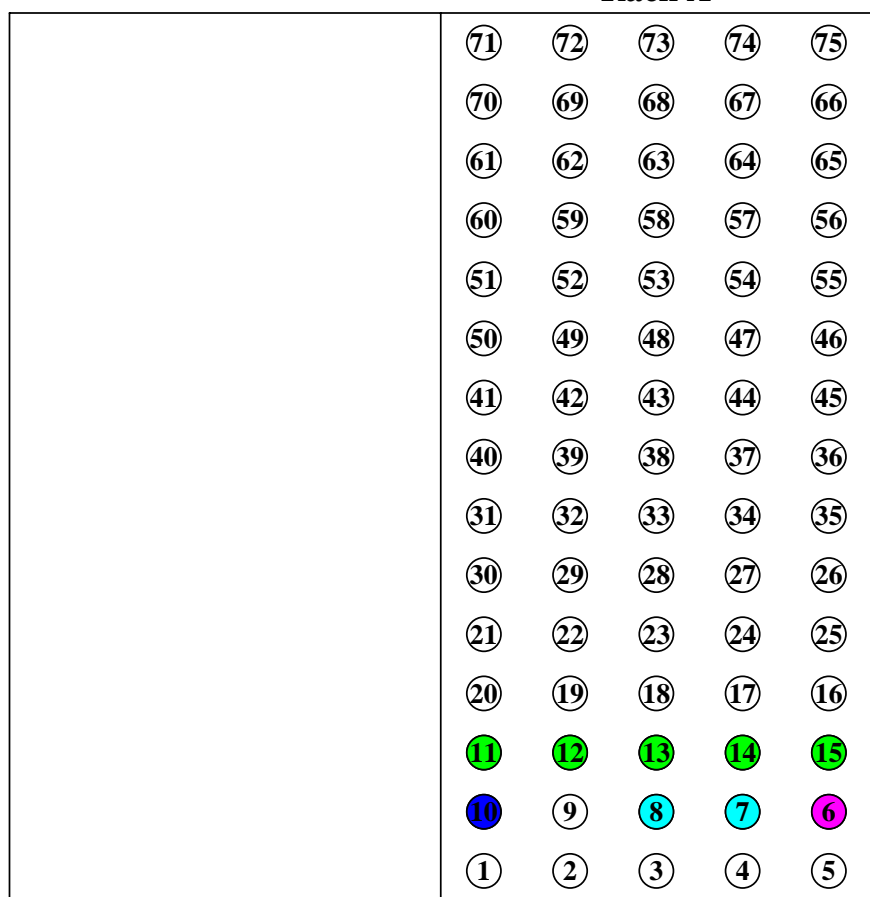

Start Tube

End Tube

Peak \#

A:6

A:7

A:10

A:11

\begin{tabular}{|c|c|c|c|}
\hline $\begin{array}{l}1 \\
2 \\
3 \\
4\end{array}$ & & $\begin{array}{c}\text { A:6 } \\
\text { A:7 } \\
\text { A:10 } \\
\text { A:11 }\end{array}$ & $\begin{array}{c}\text { A:6 } \\
\text { A:8 } \\
\text { A:10 } \\
\text { A:15 }\end{array}$ \\
\hline Duration & $\% \mathrm{~B}$ & Solvent A & Solvent B \\
\hline $\begin{array}{c}0.0 \\
1.0 \\
11.0 \\
2.0 \\
0.0 \\
1.0\end{array}$ & \begin{tabular}{|c|}
0.0 \\
0.0 \\
100.0 \\
100.0 \\
0.0 \\
0.0
\end{tabular} & $\begin{array}{l}\text { A1 hexane } \\
\text { A1 hexane } \\
\text { A1 hexane } \\
\text { A1 hexane } \\
\text { A1 hexane } \\
\text { A1 hexane }\end{array}$ & $\begin{array}{l}\text { B1 ethyl acetate } \\
\text { B1 ethyl acetate } \\
\text { B1 ethyl acetate } \\
\text { B1 ethyl acetate } \\
\text { B1 ethyl acetate } \\
\text { B1 ethyl acetate }\end{array}$ \\
\hline
\end{tabular}


Compound [36]

Sample: ALC-B-120-3

4x Column: 3

RediSep Column: Silica 4g

Flow Rate: $18 \mathrm{ml} / \mathrm{min}$

Equilibration Volume: $33.6 \mathrm{ml}$

Initial Waste: $0.0 \mathrm{ml}$

Air Purge: 10.0 min

Solvent: A1 hexane

Solvent: B1 ethyl acetate

\section{Rf 200}

Thursday 13 August 2015 02:10PM

Peak Tube Volume: Max.

Non-Peak Tube Volume: Max.

Loading Type: Solid

Wavelength 1 (red): 254nm

Peak Width: 30 sec

Threshold: 0.20 AU

Wavelength 2 (purple): 280nm
All Wavelength (orange): $200 \mathrm{~nm}-360 \mathrm{~nm}$

Peak Width: $30 \mathrm{sec}$

Threshold: 0.20 AU

Run Notes:

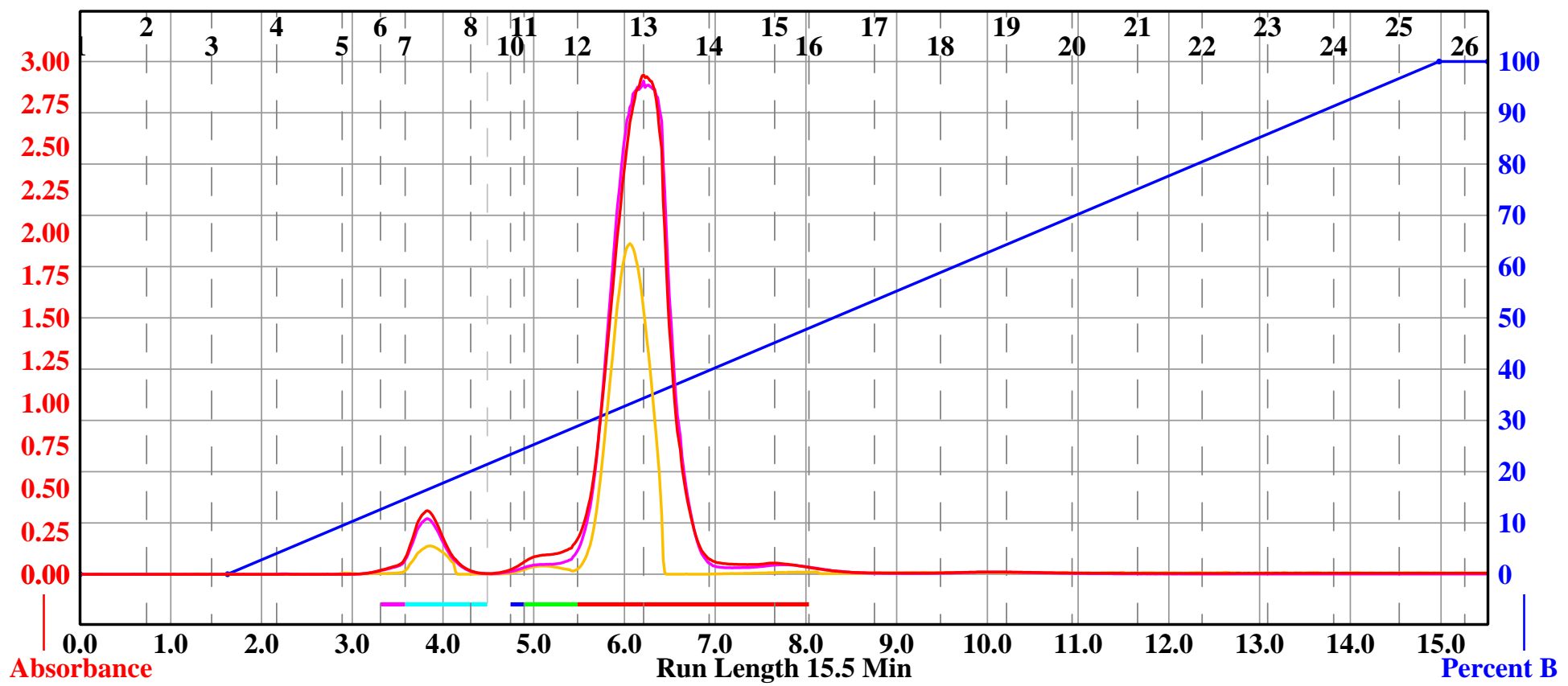

Rack A

Peak

Start Tube

End Tube

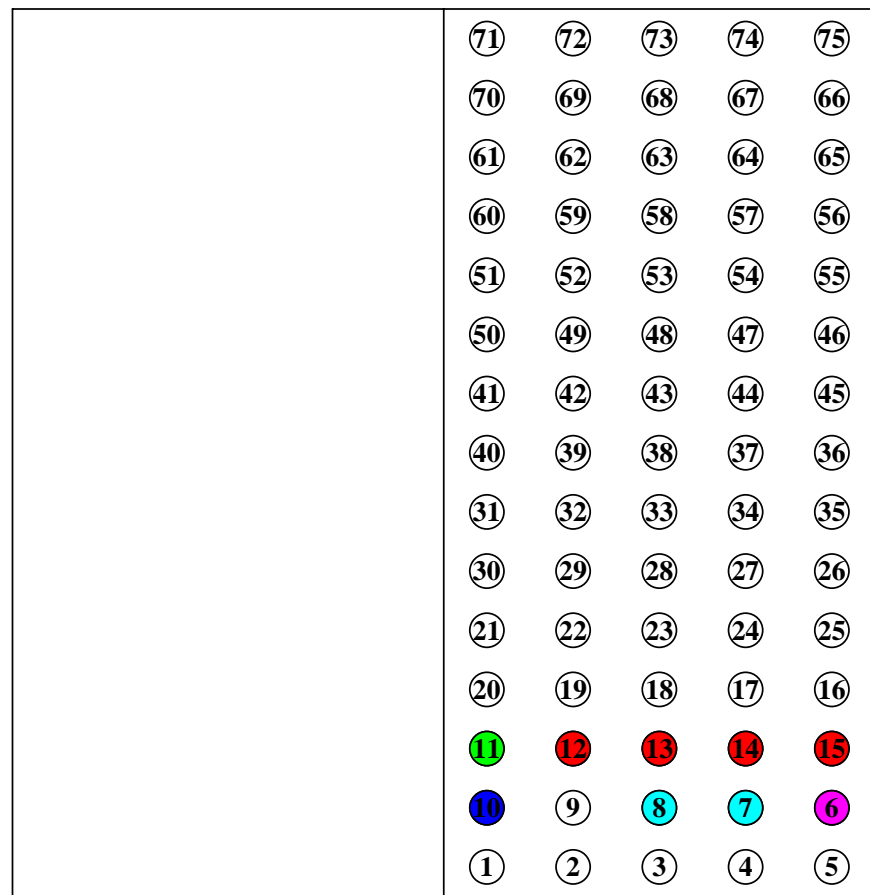

\begin{tabular}{|c|c|c|c|}
\hline $\begin{array}{l}1 \\
2 \\
3 \\
4 \\
5\end{array}$ & & $\begin{array}{c}\text { A:6 } \\
\text { A:7 } \\
\text { A:10 } \\
\text { A:11 } \\
\text { A:12 }\end{array}$ & $\begin{array}{c}\text { A:6 } \\
\text { A:8 } \\
\text { A:10 } \\
\text { A:11 } \\
\text { A:15 }\end{array}$ \\
\hline Duration & $\% \mathrm{~B}$ & Solvent A & Solvent B \\
\hline $\begin{array}{c}0.0 \\
1.6 \\
13.4 \\
0.5\end{array}$ & $\begin{array}{c}0.0 \\
0.0 \\
100.0 \\
100.0\end{array}$ & $\begin{array}{l}\text { A1 hexane } \\
\text { A1 hexane } \\
\text { A1 hexane } \\
\text { A1 hexane }\end{array}$ & $\begin{array}{l}\text { B1 ethyl acetate } \\
\text { B1 ethyl acetate } \\
\text { B1 ethyl acetate } \\
\text { B1 ethyl acetate }\end{array}$ \\
\hline
\end{tabular}


Compound [37]

Sample: ALC-B-64-1

4x Column: 2

RediSep Column: Silica 4g

Flow Rate: $18 \mathrm{ml} / \mathrm{min}$

Equilibration Volume: $33.6 \mathrm{ml}$

Initial Waste: $0.0 \mathrm{ml}$

Air Purge: 10.0 min

Solvent: A1 hexane

Solvent: B1 ethyl acetate

\section{Rf 200}

Friday 10 July 2015 10:51AM

Peak Tube Volume: Max.

Non-Peak Tube Volume: Max.

Loading Type: Solid

Wavelength 1 (red): 254nm

Peak Width: 30 sec

Threshold: 0.20 AU

Wavelength 2 (purple): 280nm
All Wavelength (orange): $200 \mathrm{~nm}-360 \mathrm{~nm}$

Peak Width: $30 \mathrm{sec}$

Threshold: 0.20 AU

Run Notes:

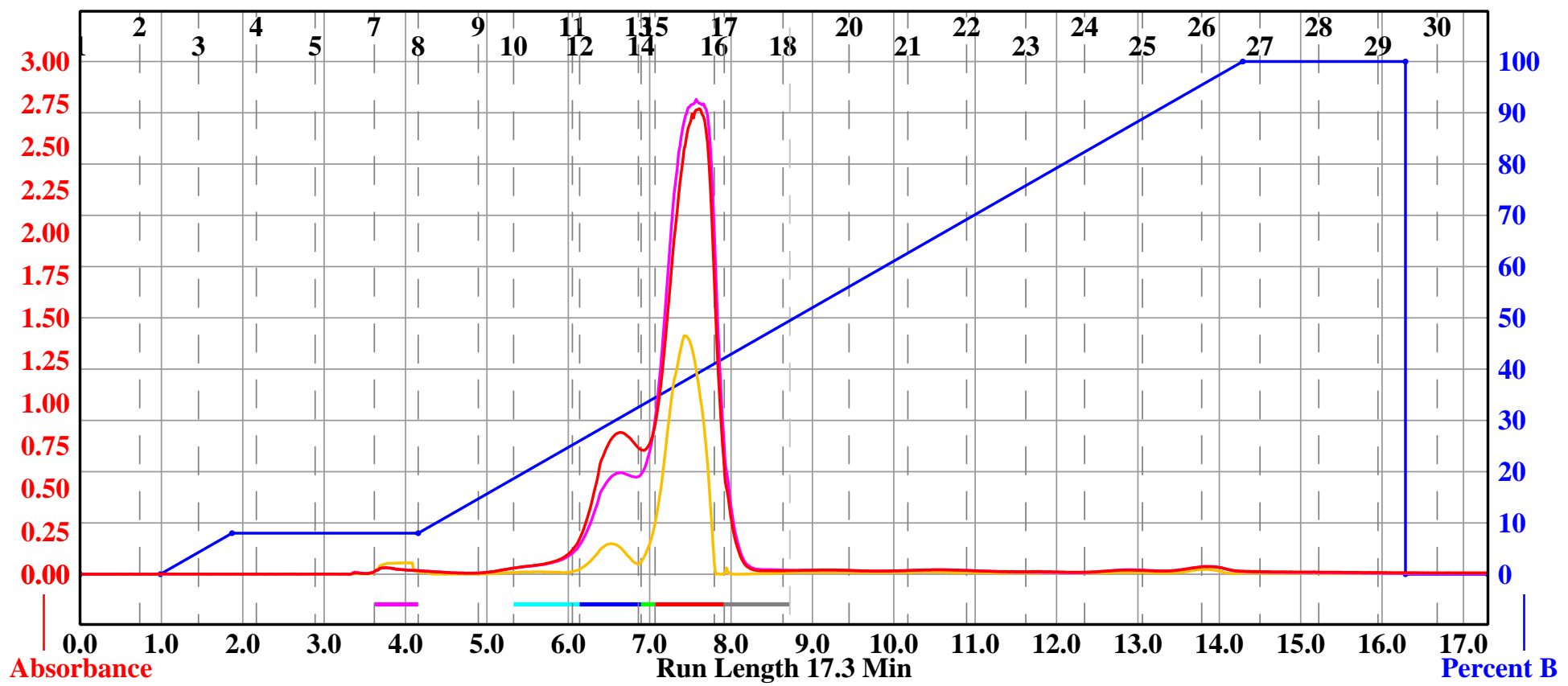

Rack A

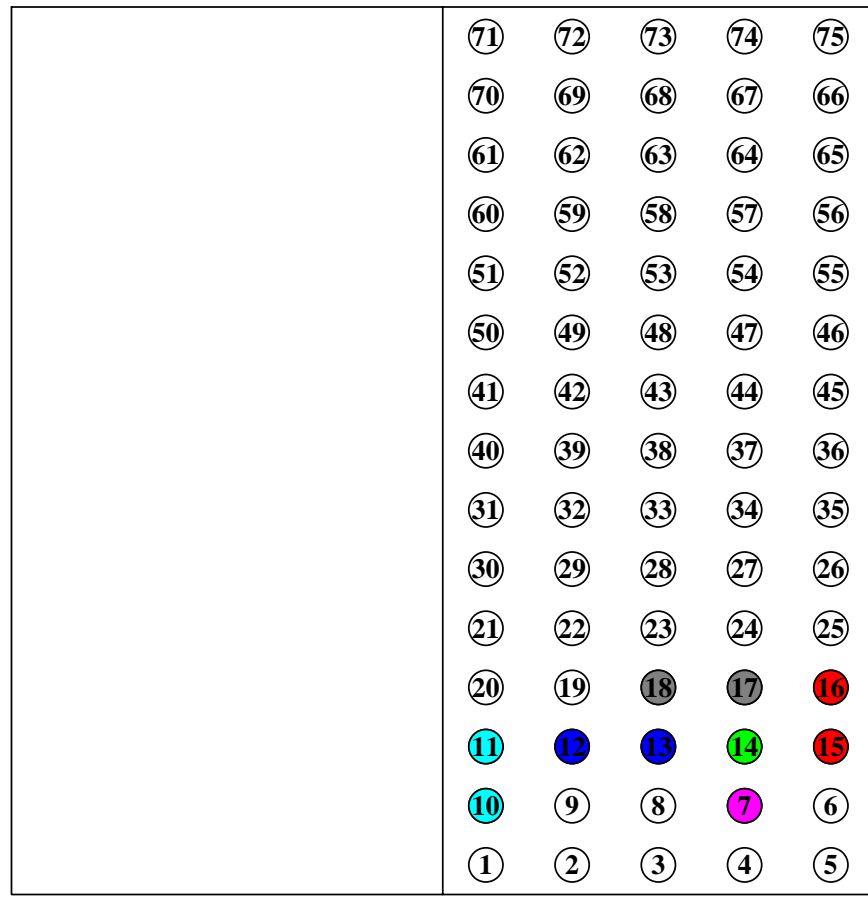

Start Tube

End Tube

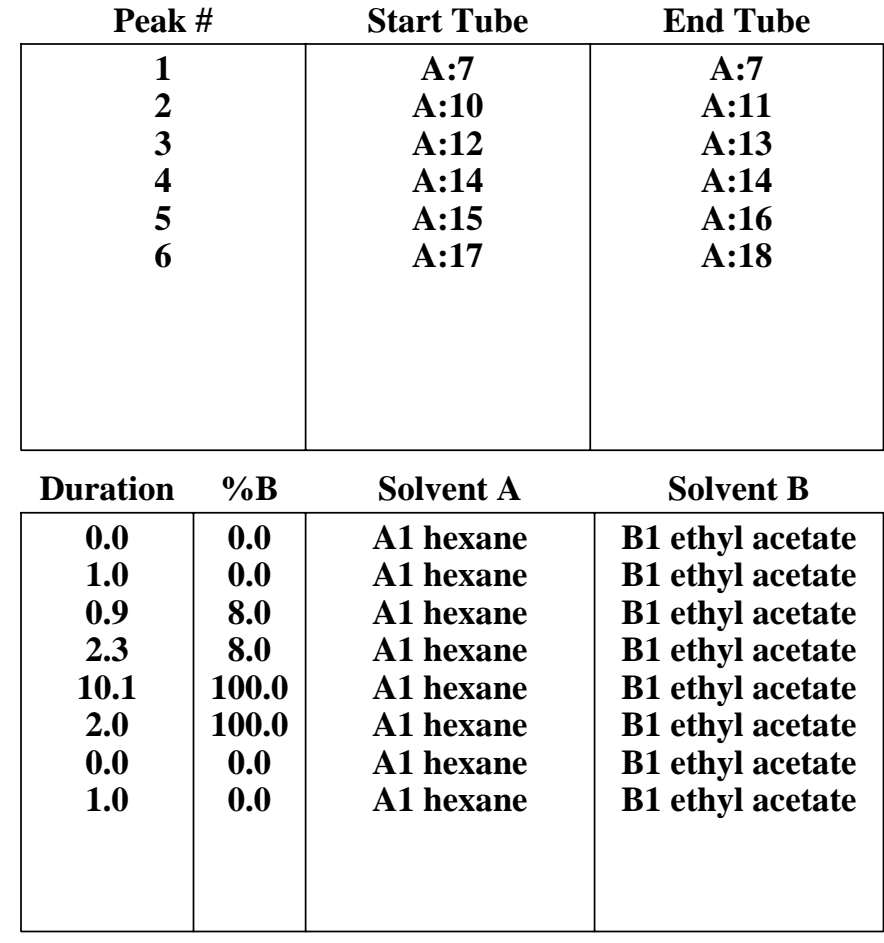

